\title{
FLEXIBLE AND FIXED TARGET STABILISATION IN AN OPEN ECONOMY
}

\author{
BY \\ ROBERT BRUCE CAMPBELL
}

A thesis submitted for the degree of Doctor of Philosophy of the Australian National University.

February, 1978 
THE AOSTPRLA

* Ligrary

Tomal anmely 
Unless otherwise indicated in the body of the text, the following study is the original work of the author. No portion of this study has been written in conjunction with another person. 


\section{ACKNOWLEDGMENTS}

I wish to thank a number of people who provided assistance during the execution and writing up of this research project. I, of course, remain entirely responsible for the results and contents. Firstly, I wish to thank my thesis supervisor, Professor S.J. Turnovsky, and Dr A.J. Preston who kindly made available important, helpful material in its unpublished form. Secondly, thanks are due to Dr G.C. O'Brien for initially interesting me in control theory and for providing me with the computing background necessary for the completion of the project. I would also like to thank the Australian Treasury and the participants of the Sixth Conference of Economists, Hobart, 1977, for providing some helpful suggestions for improving earlier versions of material contained in the thesis. Finally, I would like to thank Joan O'Neill who expertly typed the first draft and the bulk of the lengthy final draft with the assistance of Kathy Kendall. 
ABSTRACT

The underlying theme of this study is to examine and compare the effectiveness and consequences of implementing two alternative stabilisation techniques in an open economy. The study incorporates an applied extension of recent theoretical stabilisation developments. In particular, the familiar optimisation or flexible target technique is compared with the new dynamic Tinbergen or fixed target technique. Essentially, the fixed target approach allows the policy-maker to exactly achieve a set of targets over a specified time horizon when he has more targets than instruments as long as he is prepared to anticipate the target path. On the other hand, the flexible target approach imposes the constraint of compromising all targets when the number of targets exceeds the number of instruments. In order to compare the two techniques, a simple open model of the Australian economy is constructed and estimated. The model assumes a fixed exchange rate and allows for an endogenous money supply and interaction and feedback between the income, monetary and open sectors. The interrelationship between sectors complicates the use of policy and the carrying out of flexible and fixed target simulation experiments gives insight into this problem. Uncertainty is stressed throughout the study and it is found that additive uncertainty can seriously affect the mix of policy required, given a specific set of objectives. In particular, it is shown that a significant switch in the 
emphasis on the use of instruments can occur when we move from a situation of certainty to uncertainty. The switching effects are especially important in an internal and external balance framework. In addition, problems of instrument instability in an open economy are examined and specific problems faced by the applied control theorist are identified along with suggested solutions.

The applied experiments mainly focus on the targets of internal and external balance coupled with monetary targets such as the rate of interest and the money supply. The optimal achievement of monetary targets is also examined in some detail with the general conclusion that the supply of money is the appropriate target to aim for. One general conclusion is clear from the internal and external balance experiments and the monetary experiments. The achievement of target configurations which include targets from different sectors and given a fixed exchange rate regime, requires a rigorous and strong use of both monetary and fiscal policy and indeed, the exact - achievement of a target or targets under these conditions places considerable stress on the instruments. This feature results in the fixed target technique being vastly inferior to the flexible target technique given the model and the designated target paths set out in this study. 


\section{TABLE OF CONTENTS}

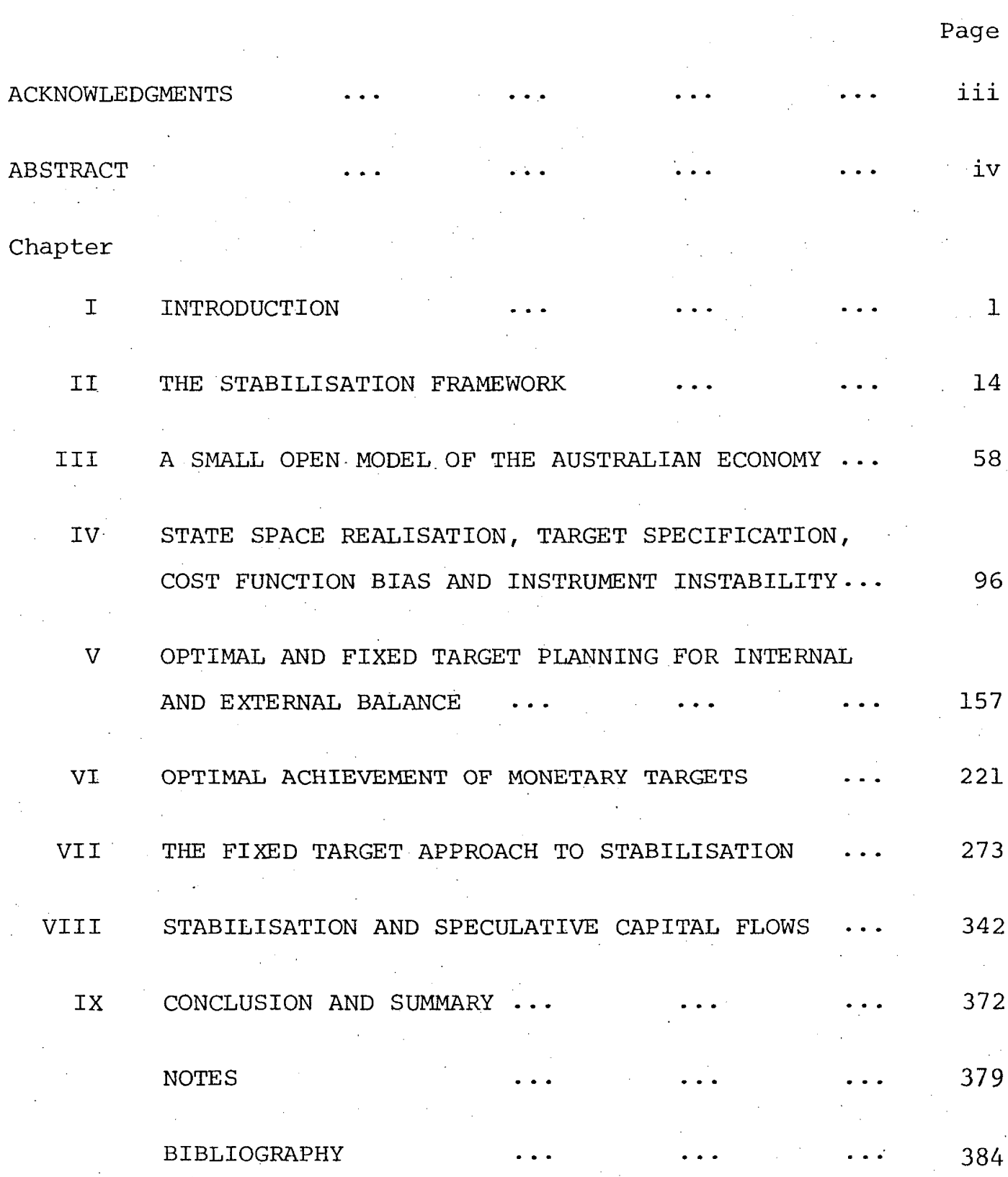




\section{CHAPTER I}

\section{INTRODUCTION}

The problem of macroeconomic stabilisation has been a topic on which a considerable degree of attention has been focussed for a considerable number of years. Recent developments in econometrics and control theory have enabled economists to approach the problem of stabilisation in a very sophisticated way, certainly at a level far above that conceived by Keynes (1935) in his classic "The General Theory of Employment, Interest and Money" which is regarded by many to be the precursor of modern macroeconomic stabilisation theory. Keynes of course did not offer any formal techniques for stabilising an economic system but his work did influence some important developments in that area. Unfortunately, the formal analysis of stabilisation techniques developed in two separate areas. The first major step in the development of formal dynamic stabilisation technigues (as opposed to purely judgemental procedures) was not the early work of Phillips as is supposed by many people, but the recognition by Tustin (1951), (1953) that the economy depicted as a simple Keynesian structure, could be formulated as a classical control system resembling a control engineer's view of a physical system. While the publication of Tustin's work was very close in

- time to that of Phillips', it is interesting to note that Tustin's view of the economy as a control system was formulated as early as 1946. While Tustin was successful in identifying the economy as a system in which controls (instruments) could be manipulated in a systematic way to affect particular responses in the outputs (targets), he failed to extend his analysis to modelling the economy within a particular structure and then analysing the nature of control policies to achieve particular output configurations. The seminal work of Phillips (1954), (1957) extended the 
frameworks of Keynes and Tustin to the application of classical control techniques to dynamic economic models which gave birth to a new literature in dynamic stabilisation. The proportional, derivative and integral policies of Phillips can be seen as a natural extension of Keynes' initial recognitiion of the need for governments to manipulate economic controls in order to achieve some desired state of the economy and when combined with the newly developing field of econometrics, presented a seemingly formidable tool for economic stabilisation.

While Phillips was essentially concerned with the dynamic evolution and stabilisation of systems, Tinbergen (1952) developed a set of propositions which, given a linear description of the model structure, would enable a policy-maker to exactly hit a set of specified targets so long as a static framework was employed. While Tinbergen's work made a considerable impact on economic thinking it was Phillips' approach which was regarded as the most amenable to practical implementations as most economists and policy makers view the world as a dynamic entity. As such, Tinbergen's static analysis was not seen to be able to cope with practical problems of macroeconomic stabilisation and the fruitful ideas put forward by Tinbergen were not built upon or extended into a formal dynamic framework until recently, as we shall see below. However, Hansen (1958, (1968), recognised the means by which extension of Tinbergen's static analysis could be carried but was unable to provide the necessary framework and his work remained largely unnoticed.

\footnotetext{
"If then we consider each target in each period as a single target (a balanced foreign trade for each year during the five year period is thus considered five single targets), and we consider each change of an instrument a measure, Rule No. 1, will be: The number of measures to be taken for the five years shall be equal to the number of targets. But the number of instruments used
} 
may be smaller because a certain instrument may be varied each year. With respect to coordination certain modifications are also necessary. So too we remark that in principle all the measures necessary may be taken at the beginning of the five year period, or they may be spread out over the single years." (Hansen (1968), p.34).

Hansen's observation provides the basic foundation for a major portion of this study.

As attractive as Phillips' policy specifications were, there were a number of problems associated with them. Firstly, the early work was conceived within a single instrument single target framework and the theory was never adequately generalised to a multi-dimensional framework as was 'Tinbergen's static formulation and Hansen's observation on a dynamic type Tinbergen framework. Secondly, the nature of the classical design procedure is essentially ad hoc with little guide being given to the policy maker on how to select a particular policy rule over another or how to choose the speed of adjustment coefficients which form a fundamental component of Phillips' approach. Furthermore, Phillips (and later Baumol(1970)) demonstrated how the incorrect implementation of policy could lead to severe oscillations and destabilising influences on income. With the development of modern optimisation techniques, particularly the work of Pontryagin (1962), the focus of attention in regard to dynamic stabilisation shifted to optimal control, particularly to the linear/ quadratic (flexible target) problem, which has become familiar in the economic literature. While Theil (1964), was one of the earliest exponents of the use of linear/quadratic techniques, it was not until the work of Pindyck (1973a) and Friedman (1972) that sufficient attention was focussed on numerically controlling economic systems to stimulate a wide body of research to be carried out in that area. Pindyck and Friedman certainly ? 
were not the first to introduce optimal control techniques into economics as it already had become a familiar technique in the growth theory literature (see Shell (1967)). Nonetheless, they demonstrated the considerable degree of knowledge that could be obtained from numerical analysis. Theoretical analysis is also important (for example see Turnovsky (1973) (1974) (1975)) but is only viable in terms of mathematical tractability for small simple examples and hence the majority of applications of optimal techniques have been to numerical models. In a recent survey article Kendrick (1976) cites over fifty applications. The use of optimal techniques were seen as a distinct advantage over the Phillips type policies in that they were more general and allowed for the specification of specific targets to which the system is to be steered. Optimising techniques were also (and still are) seen as a significant improvement over the Tinbergen instrument/target equality condition in that policies could be found to exist when the number of targets exceeded the number of instruments and the framework was dynamic rather than static. (It was in fact recognised that if the number of targets equalled the number of instruments then it was possible to hit the desired targets in a dynamic framework. Little attention was paid to a formal analysis of the existence of policy however). In fact, optimisation techniques have in many cases been justified on the grounds that if the number of targets did exceed the number of instruments available then a Tinbergen type framework was inapplicable and the best the policy maker could do would be to trade off or compromise all of his targets. Indeed, with the exception of Hansen's unrecognised observation, it was and still largely is, the accepted view that Tinbergen's static framework could not be generalised to a dynamic framework, 
"The framework (Tinbergen's) does not appear to have any clear application to dynamic analysis in which each variable has not a one-dimensional fixed value but a time pattern of behaviour." (Culbertson (1968, p.394).

Recently, Preston (1974) has, using modern control theory concepts of system controllability, successfully extended Tinbergen's static analysis into a dynamic framework thus resurrecting Tinbergen's analysis as a theoretically viable dynamic stabilisation technique and providing the formal criteria which eluded Hansen. The controllability conditions set out by Preston raise the interesting possibility that a multi-target system can be exactly stabilised over time with only one instrument varying. The practical implications of this are somewhat clouded in Preston's original paper as a continuous time formulation was employed. The basic result has been reformulated by Preston and Sieper (1977) in which a discrete time dynamic analogue to Tinbergen's famous counting rule has been derived, with the discrete time framework allowing for considerably more insight into the problem than the continuous time approach. It is shown that given policy existence (see Chapter Two), a policy maker is able to exactly hit a sequence of targets over time when the number of targets exceeds the number of controls provided the policy-maker is willing to anticipate his target path. The notion of target anticipation refers to the requirement that a policymaker can begin to manipulate his instruments now in order to hit and stay on target at some time period t. The analysis as we shall see depends crucially on the number of targets selected, the length of the desired target path and the length of the anticipation period. By treating each instument at different points of time as a separate "instrument" (as was suggested by Hansen) a dynamic counterpart to Tinbergen's static counting rule can be obtained which states that the number of time indexed instruments must equal the number of time indexed targets. This treatment 
of. instruments also accounts for the continuous time result whereby multiple targets could be achieved with a single instrument. The development of a dynamic Tinbergen framework should be viewed as the next stage of development after optimal stabilisation and finally draws together the early dynamic approach instigated by Phillips and the early static work of Tinbergen into a unified framework and in fact it poses a challenge to the continued use of optimising techniques. Optimising techniques by their very nature imply complete impatience by the policy-maker (more targets than instruments) in trying to achieve his targets, that is, he is not prepared to wait and tries to achieve them without any anticipation with the result that no targets are achieved. The dynamic Tinbergen framework (fixed targets) allows exact fine tuning when there are more targets than instruments, and indeed, raises the question of whether or not under these circumstances optimisation will become redundant. (If costs are placed on controls then of course the flexible target approach will still be relevant and the fixed target procedure will be inapplicable). A major aim of this study will be to provide the first applied evidence concerning the use of a dynamic fixed target technique for macroeconomic stabilisation and in doing so comparing it with conventional linear/ quadratic results.

While the overall theme will be one of comparing two techniques, the study will follow several other main thrusts. Firstly, while it is common for studies which aim to illustrate various stabilisation techniques to largely ignore the economic implications involved, the economics of particular scenarios presented in the following chapters will be discussed in detail. In particular, the applied portions of the investigation will focus on specific issues associated with an open economy. The optimal stabilisation literature has in general tended to ignore problems of the 
open economy, although many of the studies which have been carried out have included open sectors of varying degree, for example Garbade (1957a) and Friedman (1975a). While open sectors have been included in the relevant econometric models, attention has usually been focussed on internal targets and stabilisation problems. The problems of open economies have not been ignored but have mainly been analysed in a comparative static theoretical framework. See for example Mundell (1962) and Helliwell (1969) to name just two. Recently, Turnovsky (forthcoming) (1977c) has analysed the behaviour of a small open economy in an optimising framework. This study seeks to extend the theoretical approach of Turnovsky to an applied framework and in doing so provide a further extension to the applied work that has been carried out in relation to closed economies. The particular aspects which are focussed upon are the linkages between the income, monetary and open sectors and the corresponding problems of achieving internal and external balance and/or monetary targets given the implications of these linkages. In addition, the choice of an optimising or non-optimising stabilisation technique may affect the choice of targets. For example, compromising targets through the use of a flexible target technique may prevent exact fine tuning but may enable all target variables to lie close to their respective desired paths and be consistent with feasible levels of the controls. On the other hand, exact fine tuning through the dynamic Tinbergen framework may result in extreme adjustment by the instruments and unstable behaviour in the non-target endogenous variables. What is gained in target performance from the linear/quadratic solution may be offset by controls which are not feasible and erratic behaviour in the remainder of the system.

Uncertainty is a key issue in the implementation of policy, regardless of whether policy is formulated using econometric models and sophisticated 
quantitative techniques or pure judgement or a combination of both. Brainard (1967) has shown how the presence of uncertainty in the form of random disturbances can lead to a situation where the policy planner can minimise the variance in his targets by using all linearly independent instruments at his disposal, even if he only has one target. In a similar vein, Turnovsky (1975) has shown how the introduction of uncertainty can result in a switch in the choice of optimal monetary instrument. The results of both Brainard and Turnovsky clearly demonstrate the importance of uncertainty in selecting an appropriate mix of policy. The work of the last two mentioned author's is in a purely theoretical framework but the implications for applied analysis are only too clear; if adequate account is not taken of uncertainty in the system then erroneous and possibly destabilising policy configurations could be implemented. The above statement appears to be a contradiction in terms for if it were possible to fully take account of uncertainty then uncertainty would no longer be a problem. However, the best policy-makers can do is to include uncertainty into econometric models in the form of random coefficients and additive disturbances and then make some policy assessment based on the distribution of those random variables. Techniques are available which enable the optimal control of an econometric model to be attempted within a stochastic framework although it should be recognised that for large models the treatment of coefficients as random variables would be largely prohibitive at the application level. No such techniques are as yet available for the fixed target framework and it will become clear in chapter two that it is unlikely that the fixed target approach will be as amenable to stochastic analysis as the flexible target framework.

Applied studies have tended to focus on the variance of target variables when uncertainty is introduced and have paid little 
attention to the behaviour of the controls, particularly to the time paths of the controls. (See Abel (1975) and Chow (1972a)). Clearly it should be of prime concern to have some idea of the sensititivy of the controls to the presence of uncertainty and the ability of the controls to adjust to uncertainty in the system. An analysis of uncertainty is even more relevant in an open economy where one could expect external shocks to filter through the domestic economy - shocks which the domestic authorities will not be able to foresee. We know from the well-known nature of the feedback control laws derived in the linear/quadratic framework (Chow(1975a) for example) that to a large extent the optimal controls so derived will be self adjusting to past additive shocks. As we shall see in the following chapter, controls derived from the dynamic Tinbergen framework are unable, with one special exception, to adjust for past shocks. This implies that exact fine tuning will not take place. Will the presence of additive uncertainty result in the abandonment of the fixed target approach for a flexible target approach bearing in mind that optimisation techniques like the fixed target technique, are unable to adjust to present shocks? This is one of the questions which the study will address itself to. In addition, the effect of uncertainty on the behaviour of the controls will be examined in detail. Due to the computational complications of incorporating random coefficients into even a medium size model, only additive disturbances will be considered in this study. The necessity of such an approach will become apparent when the fixed target procedure is discussed in the next chapter. The use of random coefficients not only poses a formidable numerical problem but the requirement that coefficients can "bounce" around over time may result in a failure of policy existence in both the flexible and fixed targets frameworks. 
problem of uncertainty for the policy planner and that is the problem of correctly forecasting future values of associated uncontrollable exogenous variables; variables which are assumed to be known with certainty in the solution of both the flexible and fixed target problems. In the case of Australia, the level of exports in any period can fluctuate considerably from any previous levels due to the seasonal nature of much of the exported produce, shifts in foreign demand and foreign exchange adjustments. Additional and related complications arise from unanticipated speculative capital flows flowing in or out of the country in anticipation of exchange rate adjustments. Difficulties could arise in trying to accurately forecast when speculation will be triggered. A failure to adequately forecast such occurrences correctly, could lead to a severely fluctuating or explosive balance of payments (level of reserves) which, if not compensated for by offsetting domestic monetary policy for example, could have severe consequences for the supply of money. Allowing for wealth effects in consumption it is clear that widely divergent movements in the money supply could have destabilising effects on income. The sensitivity of optimal controls to forecast errors in exogenous variables has largely been ignored in the applied optimal stabilisation literature and of course has not even been considered within the new fixed target framework. The present study will attempt to correct this deficiency in relation to forecast errors concerning unanticipated speculative capital flows, although it should be remembered that the results will be largely model dependent.

AN OVERVIEW

Chapter Two presents a theoretical framework for both the flexible and fixed target techniques with a special emphasis on a comparison between the two. Attention is also focussed on the question 
of existence and uniqueness of policy, a question which has not received a sufficient degree of attention in the economic literature. The establishment of policy existence in an applied study is of crucial importance, especially if a fixed target technique is being utilised. Similarities between the existence and uniqueness criteria for both techniques of stabilisation are indicated where appropriate. Having developed a formal framework for stabilisation, Chapter Three contains a description and estimation of a small open model of the Australian economy under a fixed exchange rate regime, with particular emphasis on the linkages between the open, monetary and income sectors and which demonstrates the importance of considering the impact of fiscal policy on the monetary and open sectors, as well as the income sector, and similarly illustrates the importance of considering the effects of monetary policy on the income sector. It is also argued in Chapter Three that certain considerations need to be taken into account when constructing a model to be used for stabilisation purposes and indeed, some models and particular features of models will render those models unsuitable for stabilisation purposes. Such issues tend to be overlooked in the literature and should influence the type of model to be used.

Chapter Four presents a state space version of the model and clarifies the differences and similarities between the state space (one period lag) realisation familiar to economists and employed in the derivation of the linear/quadratic solution in Chapter Two and the state space realisation used in the fixed target approach which is more familiar to the non-economist control theorist. Desired target paths for all endogenous and control variables are presented along with a discussion concerning the appropriateness of using one set of weights in a quadratic cost function for all periods of a specified planning period. It is argued 
that unless weights are adjusted each time period to take account of targets growing over time, cost function bias can influence a solution and perhaps even destroy the original desired trade-offs. The chapter is completed by a discussion and investigation of some aspects of instrument instability - a problem which warrants attention in an exact fine tuning framework and in an attempted fine tuning framework such as the linear/ quadratic approach.

Chapters Five and Six incorporate discussions of applied stabilisation exercises with the main emphasis being on the linear/quadratic approach. Chapter Five includes a discussion of the problems associated with achieving internal and external balance given a fixed exchange rate and compares the flexible and fixed target approaches under suitable conditions. Problems of hitting monetary targets such as the rate of interest and the supply of money are considered in Chapter Six. The complications resulting from the presence of an open sector in following such a course of action are discussed along with the effects of uncertainty a factor which is also stressed in Chapter Five. Both chapters extend the instrument instability analysis of Chapter Four and in particular it is shown that the presence of uncontrollable exogenous variables and desired target paths which grow over time can give a mistaken impression of instrument instability when in fact the underlying structure of the model does not contain such a property. The emphasis in Chapter Seven is switched from the linear/quadratic framework to the dynamic Tinbergen fixed target framework. The applied analysis is developed through the computation of the dynamic multipliers of the system using the state space realisation of Chapter Four. Controllability matrices for both target point and target path problems are analysed for a number of target configurations. Where appropriate, the results of Chapters Five and Six 
are compared with fixed target solutions and the comparison is extended to incorporate internal and external balance targets with monetary and other income targets. The augmented target sets are then examined in both fixed and flexible target frameworks.

Foreign. exchange speculation is a problem that can easily arise under a fixed exchange rate such as that which has operated in Australia in various degrees. The result of speculation is usually to force a substantial shift in the exchange rate with the direction of the shift depending on the direction in which reserves are moving. Speculation has played a significant role in Australia's external sector in recent years with a substantial revaluation in 1972 and a severe devaluation in 1976 . At the time of writing (1977), opinion was beginning to form in favour of a further major adjustment. The formation of such an opinion of course necessarily contributes to the speculation and the subsequent exchange rate adjustment. Chapter Eight examines the effect of speculation within the context of the model and more importantly, examines the sensitivity of stabilisation techniques to speculation. It will be seen in the next chapter that in the linear/quadratic framework the optimal controls will be able to adjust to past disturbances in the system which raises the question of whether or not the automatic control laws will be able to adequately compensate for unanticipated capital flows (speculation). In contrast, the dynamic Tinbergen approach does not allow for selfadjustment in the controls which suggests that if the speculation is particularly severe then planning and fine tuning would need to be abandoned in favour of short run discretionary policy. The applied analysis will yield some insight into these issues. Chapter Nine summarises the main results and contains the conclusions of the study in addition to highlighting some areas of future research suggested by the present study. 


\section{CHAPTER II}

\section{THE STABILISATION FRAMEWORK}

In this chapter the control framework for the experiments that follow, is set out with particular emphasis on a comparison between the flexible target approach (linear/quadratic) and fixed target approach (dynamic Tinbergen). Both techniques have received attention elsewhere, particularly the linear/quadratic approach, while the fixed target approach is a relatively new technique. The linear/quadratic technique is of the following form. We seek to minimise a cost function given by

$$
\left.J=E_{0}\left[\sum_{t=1}^{T}\left[x_{t}-\bar{x}_{t}\right) \cdot Q_{t}\left(x_{t}-\bar{x}_{t}\right)+\left(u_{t}-\bar{u}_{t}\right) \cdot R_{t}\left(u_{t}-\bar{u}_{t}\right)\right]\right]
$$

subject to the system dynamics

$$
x_{t}=A x_{t-1}+C u_{t}+D z_{t}+a_{0}+\varepsilon_{t}
$$

with given initial condition

$$
x_{0}=\psi
$$

with (2.2) being a first order or state space realisation of the conventional reduced form of an econometric model. A further discussion of state space systems follows in chapter four but for the present it suffices to say that the state vector, $x_{t}$, consists of the output vector, $y_{t^{\prime}}$ or vector of endogenous variables contained within the system of interest, plus newly defined variables to take account of endogenous variables with lags greater than one period and lagged controls and exogenous variables. The notion of states and outputs will be crucial in the development of both the 
optimal and non-optimal fixed target approaches to stabilisation. The matrices A, C and D are constant matrices of coefficients of lagged state, control and exogenous variables respectively while $u_{t}$ and $z_{t}$ are vectors of control and exogenous variables. $a_{0}$ is a vector of constant terms and $\varepsilon_{t}$ is a vector of random additive disturbances with zero mean and satisfying a specified covariance matrix. In relation to the cost function (2.1), $\bar{x}_{t}$ and $\bar{u}_{t}$ are vectors of desired or target values of the state variables (specifically the output component of the current state) and desired values of the control variables. $\mathrm{E}_{\mathrm{O}}$ is the expectation conditional on the initial conditions and $Q_{t}$ and $R_{t}$ are cost matrices which penalise deviations of the state variables and control variables from their respective target paths. Usually, $Q_{t}$ is specified to be positive semi-definite while in many cases it is convenient to specify $R_{t}$ as a positive definite matrix. This restriction on $R_{t}$ implies that there is some positive cost (however small) associated with deviations of all control variables from their desired paths. The imposition of this restriction on $R_{t}$ is not necessary to obtain a mathematical solution without resorting to the complications of using a generalised inverse but, as we shall see below, it is when combined with other conditions, sufficient to ensure that a policy exists and is unique. Before proceeding to a discussion of the solution technique it is convenient to introduce the following notational simplification. In order to apply control theory of the fixed or flexible target variety in the absence of any learning procedure, it is necessary to assume that the values of the uncontrollable exogenous variables are known, or are believed to be known, for each time period, $;$ a highly restrictive assumption, which will be relaxed in Chapter Eight, in relation to correctly forecasting the "trigger" level of foreign reserves which will set off speculative capital flows. Thus, 
$\mathrm{Dz}_{t}$ and $\mathrm{a}_{\mathrm{o}}$ can be added together and treated as given over the planning period. To achieve thïs a new vector, $b_{t}$ ' can be defined such that

$$
\mathrm{b}_{t} \equiv \mathrm{Dz} t+\mathrm{a}_{0}
$$

(2.2) can now be written as

$$
x_{t}=A x_{t-1}+C u_{t}+b_{t}+\varepsilon_{t}
$$

The reduction of $(2.2)$ to (2.5) will greatly facilitate the derivation of the optimal control laws. Letting $\mathrm{n}$ equal the number of state and $\mathrm{k}$ equal the number of control variables yields the following system dimensions. A and $C$ are ( $n x n)$ and (nxk) matrices respectively while $x_{t}, x_{t-1}, \quad b_{t}$ and $\varepsilon_{t}$ are all:(nxl) vectors. $u_{t}$ is $(k \times 1)$.

The specification of the optimal control problem as it is presented above is certainly the most basic specification and the literature, both economic and non-economic, has progressed significantly from the formulation. However, in terms of the aims of this study the formulation is quite acceptable. The use of a quadratic cost function can be criticised on many grounds (see theil (1964)) particularly from the point of view of the restrictive way in which penalties are allocated. It is argued, with some justification, that policy-planners are in many instances only concerned with particular target variables lying between certain specified upper and lower bounds or above or below particular limited, in addition of course to normal quadratic treatment. This type of problem can be adequately handled by a piece-wise quadratic approach (Friedman (1975), Fitzgerald, Johnston and Bayes (1973)). The piece-wise quadratic approach has not been adopted here as has been stated in the previous chapter, the underlying theme of this study is to consider system performance under exact fine tuning or attempted fine tuning. The fixed 
target approach yields exact fine tuning after an appropriate policy lead while the linear/quadratic approach attempts in the absence of an exact fine tuning capability, to come as close as possible to a fine tuning solution given the constraints of the system and possible tradeoffs between targets. The adoption of a piece-wise quadratic approach implicitly assumes that policy-planners are not concerned with getting as close as possible to levels of specified targets. Indeed, if policyplanners were not concerned with trying to exactly achieve targets then the fixed target approach would be redundant. Because of the underlying philosophy of the quadratic cost function approach to optimal control, it is logical that this should be the optimal technique to be used as a comparison with the fixed target approach - at least at this stage of our knowledge of the performance of a system under dynamic fixed target control which up until this study was nil. There is ample scope for future research into comparative techniques.

A second objection to the optimal control formulation outlined above is that it is linear and stochastic only in relation to the inclusion of the additive disturbances. The linearity restriction is probably the restriction which has caused the greatest degree of objection to optimal control techniques, especially in the initial stages of the development of macro economic applications, resulting in accusations of "fixing" the models and research problems to fit the technique. In response to such a criticism a researcher has available a wide variety of techniques to solve non-linear optimisation problems, for example, Chow (1976a), (1976c), Livesey (1971), Garbade (1975a) and Gupta et al (1975) to name just a few. While attempts to find non-linear techniques are commendable in themselves, it must be pointed out that such techniques involve approximations. The question that has not been investigated is 
whether or not keeping a model in its non-linear form and approximately optimally controlling it (which usually involves a linearisation during the procedure) or proceeding directly to a linear estimation and exactly controlling the model will give widely divergent solutions. That is, is there anything to be gained from trying to approximately control a nonlinear model which could not be similarly achieved by using a linear version of the system. ${ }^{1}$ On a more practical level, as the fixed target framework has only been developed for linear systems it would appear to be most sensible, at least initially, to use a linear optimal control technique as a comparison. As we shall see below, the fixed target solution makes use of the dynamic final form multipliers of the system and while the use of a non-linear model may be reasonably adequate in the flexible target solution, the linearisation of the model for solution in the fixed target framework may result in unacceptable solutions being obtained due to the linearised estimates of the final form multipliers behaving in a way which is contrary to the underlying non-linear structure. Such a result was obtained by Friedman (1975) in his linearisation of the Wharton Model.

The solution to the linear/quadratic problem has received adequate attention elsewhere, see for example Chow (1970), (1972a), (1973b),(1975a), Pindyck (1973a) and Turnovsky (1974), (1977b) and as such will not receive an excessively detailed exposition here. The solution to the problem is obtained by the application of Bellman's (1957) Principle of optimality which states that in a multi-dimension decision process an optimal solution for the entire process can be obtained by computing an optimal solution for each stage of the process. The set of all optimal decisions then consistute an optimal solution for the entire multi-decision problem. To employ this principle it suffices to find a 
general solution which is optimal for each stage (time period) of the policy decision process. It must be pointed out that the problem can be solved in a number of ways; for example by the use of Lagrange multipliers or by Pontryagins (1962) Maximum Principle. In either case the solution will be of the same general form as that obtained by dynamic programming. ${ }^{2}$ The problem as specified by (2.1) - (2.3) differs slightly to the conventional formulation of the problem in the economic literature. For example, the instruments and instrument costs are included as a separate component of the cost function which differs from the approach adopted by Chow in any of his papers cited above. Chow includes $u_{t}$ in $x_{t}$ and hence $\bar{u}_{t}$ and $R_{t}$ disappear from the solution and are incorporated in $\bar{x}_{t}$ and $Q_{t}$. The inclusion of the controls in the cost function as a separate component follows the usual practice of the non-economic control literature and has several distinct advantages. Firstly, for very large systems it can greatly reduce the size of the state space system to be controlled and will alleviate the "curse of dimensionality" which is associated with dynamic programming techniques. This will become apparent in Chapter Four where the state space realisation is discussed in more detail. Secondly, it enhances our intuition and insight into how the optimal solutions will respond to the relative adjustments in state and control costs and the impact, for example, of uncontrollable exogenous variables on the optimal control vector given various state and instrument weightings. The second observation leads into a third related advantage in that our insight into existence and uniqueness criteria associated with linear/quadratic optimal control, is considerably enhanced, particularly for those wo do not profess any great expertise in this, area. A summary of existence and uniqueness conditions given below illustrates this point quite adequately. Optimal control applications and theory in economics has not been solely confined 
to the Chow specification, for example, Pindyck (1973a), who treated controls as a separate component of the cost function but derived his control laws from the Maximum Principle. The theoretical work of Turnovsky (1977a) also employs a dynamic programming procedure along with a cost function of the (2.1) type but includes the exogenous variables in the vector of additive disturbances and thus a unified treatement of the linear/quadratic problem given (2.1) and uncontrollable exogenous variables has not gained any predominance in the economic literature although it should be noted that the changes required to the equations specifying the computation of the relevant feedback matrices in both Chow and Turnovsky are minimal. (2.1) is of course an equivalent expression for Chow's cost function which is of the form

$$
J=E_{0}\left[\sum_{t=1}^{T}\left[\left(x_{t}-\bar{x}_{t}\right) \cdot k_{t}\left(x_{t}-\bar{x}_{t}\right)\right]\right]
$$

and while the resulting computational procedure differs slightly, the two procedures are in fact equivalent and will yield precisely the same numerical answers.

The solution procedure proceeds as follows. The appropriate control laws are found by substituting (2.5) into (2.1) and obtaining an expression for the optimal expected cost at time $\mathrm{T}$ (terminal time). This procedure is repeated for the remaining time periods although it will become apparent that a generalised expression can be obtained after a minimal amount of manipulation through time periods. The equations at any time $t$, are a function of all present and future targets (specified by the weighting pattern in $Q_{t}$ and $R_{t}$ ) and future forecasts of the exogenous variables while the optimal control vector itself, is a function of the same variables through the influence of the relevant feedback matrices 
and the immediate past state of the system. The random additive disturbances are handled by appealing to the principle of certainty equivalence (Theil (1957), Simon (1956)) and can thus be eliminated from the derivation of the control laws. The certainty equivalence assumption leads to the application of the same $F_{t}$ and $f_{t}$ for all $t$ for both deterministic and additive stochastic frameworks. The general form of the solution is given by the linear feedback relationship

$$
u_{t}^{*}=F_{t} x_{t-1}+f_{t}
$$

where $u_{t}^{*}$ is the vector of optimal controls, $F_{t}$ is a feedback matrix which relates the optimal controls to the past state and can be regarded as a type of automatic response rule which adjusts controls to deviations of the state away from its desired values while $\mathrm{f}_{t}$ is an additive vector or intercept term which in addition to being a function of control costs and instrument impacts and state costs and dynamics, summarizes the influence of the uncontrollable exogenous variables on the optimal control vector. The cost function for the final period, $T$, can be rewritten as

$$
J_{T}=E_{T-1}\left[x_{T}^{\prime} H_{T} x_{T}-2 x_{T}^{\prime} h_{T}+e_{T}+u_{T}^{\prime} R_{T} u_{T}-2 u_{T}^{\prime} n_{T}+d_{T}\right]
$$

where

$$
\begin{aligned}
& \mathrm{H}_{\mathrm{T}}=\mathrm{Q}_{\mathrm{T}} \\
& \mathrm{H}_{\mathrm{T}}=\mathrm{k}_{\mathrm{T}}=\mathrm{Q}_{\mathrm{T}} \overline{\mathrm{x}}_{\mathrm{T}} \\
& \mathrm{e}_{\mathrm{T}}=\overline{\mathrm{x}}_{\mathrm{T}}^{\prime} \mathrm{Q}_{\mathrm{T}} \overline{\mathrm{x}}_{\mathrm{T}} \\
& \mathrm{d}_{\mathrm{T}}=\overline{\mathrm{u}}_{\mathrm{T}}^{\prime} \mathrm{R}_{\mathrm{T}} \overline{\mathrm{u}}_{\mathrm{T}} \\
& \mathrm{n}_{\mathrm{T}}=\mathrm{R}_{\mathrm{T}} \overline{\mathrm{u}}_{\mathrm{T}}
\end{aligned}
$$

and (2.9) and (2.10) have been defined as above in order to facilitate a 
multi-period generalisation at a later stage. Substituting (2.5) into (2.8) gives

$$
\begin{aligned}
J_{T}= & \left(A x_{T-1}+C u_{T}+b_{T}\right) H_{T}\left(A x_{T-1}+C u_{T}+b_{T}\right) \\
& -2\left(A x_{T-1}+C u_{T}+b_{T}\right) h_{T}+e_{T}+u_{T}^{\prime} R_{T} u_{T} \\
& -2 u_{T}^{\prime} n_{T}+d_{T}+E_{T-1} \varepsilon_{T}^{\prime} H_{T} \varepsilon_{T}
\end{aligned}
$$

To minimise costs at time period $T,(2.14)$ is differentiated with respect to $u_{\mathrm{T}}$ and equated to zero.

$$
\frac{\partial J_{T}}{\partial u_{T}}=2 C^{\prime} H_{T}\left(A x_{T-1}+C u_{T}+b_{T}\right)-2 C^{\prime} h_{T}+2 R_{T} u_{T}-2 n_{T}=0
$$

Solving for the optimal control $\mathrm{u}_{\mathrm{T}}^{*}$ yields

$$
\begin{aligned}
u_{T}^{*}= & -\left(C^{\prime} H_{T} C+R_{T}\right)^{-1} C^{\prime} H_{T} A x_{T-1}-\left(C^{\prime} H_{T} C+R_{T}\right)^{-1} C^{\prime} H_{T} b_{T} \\
& +\left(C^{\prime} H_{T} C+R_{T}\right)^{-1} n_{T}
\end{aligned}
$$

which, upon rearranging and grouping terms, can be rewritten as

$$
\begin{aligned}
& u_{T}^{*}=F_{T} x_{T-1}+f_{T} \\
& \text { (* denotes optimal) }
\end{aligned}
$$

where

$$
\begin{aligned}
& F_{t}=-\left(C^{\prime} H_{T} C+R_{T}\right)^{-1} C^{\prime} H_{T} A \\
& f_{T}=-\left(C^{\prime} H_{T} C+R_{T}\right)^{-1}\left(C^{\prime} H_{T} b_{T}-C^{\prime} h_{T}-n_{T}\right) .
\end{aligned}
$$

It can be seen that with the exception of the time subscripts (2.17) is in precisely the same form as that specified by (2.7). The optimal expected cost for period $\mathrm{T}$, condition on $\mathrm{x}_{\mathrm{T}-1}$ can be derived by substituting (2.17) into (2.14) giving 


$$
\begin{aligned}
& \mathrm{J}_{\mathrm{T}}^{*}=\left(A \mathrm{x}_{\mathrm{T}-1}+\mathrm{CF}_{\mathrm{T}} \mathrm{x}_{\mathrm{T}-1}+\mathrm{Cf}_{\mathrm{T}}+\mathrm{b}_{\mathrm{T}}\right)^{\prime} \mathrm{H}_{\mathrm{T}}\left(\mathrm{Ax} \mathrm{x}_{\mathrm{T}-1}+\mathrm{CF}_{\mathrm{T}} \mathrm{x}_{\mathrm{T}-1}\right. \\
& \left.+C f_{T}+b_{T}\right)-2\left(A x_{T-1}+C F_{T} x_{T-1}+C F_{T}+b_{T}\right)^{\prime} h_{T} \\
& +e_{T}+\left(F_{T} x_{T-1}+f_{T}\right) \cdot R_{T}\left(F_{T} x_{T-1}+f_{T}\right)-2\left(F_{T} x_{T-1}+f_{T}\right) \cdot n_{T} \\
& +d_{T}+E \varepsilon_{T}^{\prime} H_{T} \varepsilon_{T}
\end{aligned}
$$

Grouping and rearranging terms gives

$$
\begin{aligned}
J_{T}^{*}= & \left.x_{T-1}^{\prime}\left(A+C F_{T}\right)^{\prime} H_{T}\left(A+C F_{T}\right) x_{T-1}+2 x_{T-1}^{\prime}\left(A+C F_{T}\right)^{\prime} H_{T} b_{T}-h_{T}\right) \\
& +\left(C f_{T}+b_{T}\right)^{\prime} H_{T}\left(C f_{T}+b_{T}\right)-2\left(C f_{T}+b_{T}\right)^{\prime} h_{T}+e_{T} \\
& +\left(F_{T} x_{T-1}+f_{T}\right)^{\prime} R_{T}\left(F_{T} x_{T-1}+f_{T}\right)-2\left(F_{T} x_{T-1}+f_{T}\right)^{\prime} n_{T}+d_{T} \\
& +E \varepsilon_{T}^{\prime}{ }_{T} \varepsilon_{T}
\end{aligned}
$$

Having determined the optimal control for period $\mathrm{T}$ along with the corresponding optimal expected cost, we are now in a position to consider period $T-1$. The total welfare cost for period $T-1$ will include the costs incurred in period $\mathrm{T}-1$ plus the optimal expected cost from period $\mathrm{T}$. The total welfare cost in period $\mathrm{T}-1$ is therefore given by

$$
\begin{aligned}
J_{T-1}= & E_{T-2}\left[x_{t-1}^{\prime} Q_{T-1} x_{T-1}-2 x_{T-1}^{\prime}+x_{T-1}^{\prime} \bar{x}_{T-1}+u_{T-1}^{\prime} R_{T-1} u_{T-1}\right. \\
& \left.-2 u_{T-1}^{\prime} n_{T-1}+\bar{u}_{T-1}^{\prime} R_{T-1} \bar{u}_{T-1}+J_{T}^{*}\right]
\end{aligned}
$$

where

$$
k_{T-1}=Q_{T-1} \bar{x}_{T-1}
$$

and

$$
\mathrm{n}_{\mathrm{T}-1}=\mathrm{R}_{\mathrm{T}-1} \overline{\mathrm{u}}_{\mathrm{T}-1}
$$


Substituting (2.21) for $\mathrm{J}_{\mathrm{T}}^{*}$ in (2.22) and grouping and rearranging terms yields the following expression.

$$
\begin{aligned}
J_{T-1}= & E_{T-2}\left[x_{T-1}^{\prime}\left(Q_{T-1}+F_{T}^{\prime} R_{T} F_{F}+\left(A+C F_{T}\right)^{\prime} H_{T}\left(A+C F_{T}\right)\right) x_{T-1}\right. \\
& \quad-2 x_{T-1}^{\prime}\left(k_{T-1}+F_{T}^{\prime} n_{T}+\left(A+C F_{T}\right)^{\prime}\left(h_{T}-H_{T} b_{T}\right)\right) \\
+ & \bar{x}_{T-1}^{\prime} Q_{T-1} \bar{x}_{T-1}+u_{T-1}^{\prime} R_{T-1} u_{T-1}-2 u_{T-1}^{\prime} n_{T-1} \\
& +\bar{u}_{T-1}^{\prime} R_{T-1} \bar{u}_{T-1}+\left(C f_{T}+b_{T}\right)^{\prime} H_{T}\left(C f_{T}+b_{T}\right) \\
& -2\left(C f_{T}+b_{T}\right) h_{T}+e_{T}+f_{T}^{\prime} R_{T} f_{T}-2 f_{T}^{\prime} n_{T}+d_{T} \\
& \left.+E \varepsilon_{T T}^{\prime} \varepsilon_{T}\right] .
\end{aligned}
$$

The apparent formidable nature of (2.25) is somewhat deceiving as an appropriate grouping in terms will convert (2.25) into a function of the same general form as (2.8). Thus,

$$
\begin{aligned}
\mathrm{J}_{\mathrm{T}-1}= & \mathrm{E}_{\mathrm{T}-2}\left[\mathrm{x}_{\mathrm{T}-1}^{\prime} \mathrm{H}_{\mathrm{T}} \mathrm{x}_{\mathrm{T}-1}-2 \mathrm{x}_{\mathrm{T}-1} \mathrm{~h}_{\mathrm{T}-1}+\mathrm{u}_{\mathrm{T}-1}^{\prime} \mathrm{R}_{\mathrm{T}-1} \mathrm{u}_{\mathrm{T}-1}\right. \\
& \left.-2 \mathrm{u}_{\mathrm{T}-1}^{\prime} \mathrm{n}_{\mathrm{T}-1}+\mathrm{e}_{\mathrm{T}-1}+\mathrm{d}_{\mathrm{T}-1}\right]
\end{aligned}
$$

where

$$
\begin{aligned}
& \mathrm{H}_{\mathrm{T}-1}=\mathrm{Q}_{\mathrm{T}-1}+\mathrm{F}_{\mathrm{T}}^{\prime} \mathrm{R}_{\mathrm{T}} \mathrm{F}_{\mathrm{T}}+\left(\mathrm{A}+\mathrm{CF}_{\mathrm{T}}\right)^{\prime} \mathrm{H}_{\mathrm{T}}\left(\mathrm{A}+\mathrm{CF}_{\mathrm{T}}\right) \\
& h_{T-1}=k_{T-1}+F_{T}^{\prime} n_{T}+\left(A+C F_{T}\right) \cdot\left(h_{T}-H_{T} b_{T}\right) \\
& e_{T-1}=\bar{x}_{T-1}^{\prime} Q_{T-1} \bar{x}_{T-1}+\left(C F_{T}+b_{T}\right)^{\prime} H_{T}\left(C f_{T}+b_{T}\right) \\
& -2\left(C f_{T}+b_{T}\right) h_{T}+e_{T}+E \varepsilon_{T}^{\prime} H_{T} \varepsilon_{T} \\
& d_{T-1}=\bar{u}_{T-1} R_{T-1} \bar{u}_{T-1}+f_{T}^{\prime} R_{T} f_{T}-2 f_{T}^{\prime} n_{T}+d_{T}
\end{aligned}
$$


Taking the differential $\partial \mathrm{T}_{\mathrm{T}-1} \backslash \partial \mathrm{u}_{\mathrm{T}-1}$ and solving for $\mathrm{u}_{\mathrm{T}-1}^{*}$ will yield an optimal solution for the control vector identical to that given by $(2.16)$ and (2.17). Of course, in this case the time subscripts will be lagged one period. The process outlined above is repeated for each time period from $t=T$ to $t=1$. It should be clear from the preceding analysis that for each time period after $t=T$, the same general solution will be obtained for the optimal control vector $u_{t}^{*}$. To illustrate the general nature of the solution (2.26) is differentiałedwith respect to $u_{T-1}$ with the adjustment that the time subscript is replaced by a more general case, $t$. Solving for the optimal control after differntiating (2.26) yields the following. The optimal control is given by

$$
u_{t}^{*}=F_{t} x_{t-1}+F_{t}
$$

where

$$
\begin{aligned}
& F_{t}=-\left(C^{\prime} H_{t} C+R_{t}\right)^{-1} C^{\prime} H_{t} A \\
& f_{t}=\left(C^{\prime} H_{t} C+R_{t}\right)^{-1}\left(C^{\prime} H_{t} b_{t}-C^{\prime} h_{t}-n_{t}\right) \\
& H_{t-1}=Q_{t-1}+F_{t}^{\prime} R_{t} F_{t}+\left(A+C F_{t}\right)^{\prime} H_{t}\left(A+C F_{t}{ }^{\prime}\right. \\
& h_{t-1}=k_{t-1}+F_{t}^{\prime} n_{t}+\left(A+C F_{t}\right)^{\prime}\left(h_{t}-H_{t} b_{t}\right) \\
& h_{t-1}=Q_{t-1} \bar{x}_{t-1} \\
& n_{t-1}=R_{t-1} \bar{u}_{t-1}
\end{aligned}
$$

with boundary conditions

$$
\begin{aligned}
& \mathrm{H}_{\mathrm{T}}=\mathrm{Q}_{\mathrm{T}} \\
& \mathrm{h}_{\mathrm{T}}=\mathrm{k}_{\mathrm{T}}=\mathrm{Q}_{\mathrm{T}} \overline{\mathrm{x}}_{\mathrm{T}}
\end{aligned}
$$




$$
\mathrm{n}_{\mathrm{T}}=\mathrm{R}_{\mathrm{T}} \overline{\mathrm{u}}_{\mathrm{T}}
$$

If the cost matrices $Q_{t}$ and $R_{t}$ are held constant at $Q$ and $R$ for all time periods of the planning period, that is for $t=1$ to $t=T$, the matrices $\mathrm{F}_{t}$ and $\mathrm{H}_{t}$ will converge to steady state solutions of the form

$$
\begin{aligned}
& F=-\left(C^{\prime} H C+R\right)^{-1} C^{\prime} H A \\
& H=Q+F^{\prime} R F+(A+C F)^{\prime} H(A+C F)
\end{aligned}
$$

provided that the characteristic roots of the matrix $(A+C F)$, are less than unity in absolute terms. Very briefly, this result follows from the fact that (2.32) can be written as an infinite series of the form

$$
\begin{aligned}
H=Q & +(A+C F) \cdot Q(A+C F)+(A+C F)^{\prime 2} Q(A+C F)^{2} \\
& +(A+C F)^{\prime 3} Q(A+C F)^{3}+\ldots \ldots \cdots
\end{aligned}
$$

and that the steady state will not exist unless the series of (2.43) converges. ${ }^{3}$ For most applied optimal stabilisation studies carried out so far, it has been the practice to use only one set of weights for each experiment thus creating favourable conditions for a steady state solution (see Chow (1972a) where it is claimed that $\mathrm{F}_{t}$ converges very rapidly). However, the use of one set of weights when targets are growing over time may result in the original intended trade-off being disrupted. To maintain exact trade-offs it may be necessary to adjust weights in each time period thus ensuring that a steady state solution will not be obtained. The issues associated with this type of procedure are discussed in detail in Chapter Four. The addition of time variant system matrices in place of the constant matrices A, C and D does not affect the solution procedure or resulting control laws in the finite time horizon case although it is essential to have time invariant matrices in the infinite 
horizon case in order to ensure the appropriate convergence to a steady state solution. The imposition of time subscripts on the system matrices does provide some conceptual difficulties. In particular, if the procedure outlined above is to be used then the addition of time subscripts implies an exact knowledge about future structural shifts as the solution must be computed backwards in time. A more appropriate and realistic procedure would be to develop the optimisation problem within an adoptive or learning framework so that the optimal solution could be updated as better information about the present and future structures becomes available. (See Abel (1975)). For the purposes of this study, the system matrices will be held constant over all time periods of a particular planning horizon. The recursive nature of the optimal solution and the treatment of control costs, $\mathrm{R}_{\mathrm{t}^{\prime}}$ as an explicit component of the cost function gives rise to, and illustrates, a further interesting property. If control costs are increased relative to state costs, then the feedback coefficients of the control(s) of concern will diminish in importance with respect to the determinination of the appropriate component(s) of the control vector, $u_{t}^{*}$. The additive vector, $f_{t}$, will then become more important in relation to setting the level of the relevant controls in any particular time period. As control costs become exceptionally large, the weighted control will gradually become an open loop control with little or no feedback from $x_{t-1}$ with complete dominance of the additive vector $f_{t}$. In the limit, an open loop control will exist. If all controls are weighted heavily to produce this situation then optimisation will become unnecessary as the appropriate solution could be obtained by simulating the system with the desired fixed open loop control levels. The occurrence of the above can be very easily verified by considering a simple one control, one time period and one target problem or from a 
close inspection of the control laws. In particular, it should be observed that the term $n_{t}$ in (2.33) will dominate the solution as $R_{t}$ is increased relative to $Q_{t}$.

The format of the solution requires some comment in relation to the Chow solution and the Turnovsky solution. First of all, assuming that all costs on controls are zero, $R_{t}=0$, then by deleting $R_{t}$ and $n_{t}$ from the above control equations we can recover Chow's solution. Of course we would then need to assume that $u_{t}$ has been included in the state vector and can be weighted through the cost matrix $Q_{t} \cdot$ Similarly, by explicitly deleting the uncontrollable exogenous variables from the system and including them in the vector $\varepsilon_{t}$ and looking at state and control deviations rather than levels, we can recover the Turnovsky solution. The solution presented above is therefore quite general in its applicability and a computer program designed to solve it would also solve control problems in either the Chow or Turnovsky formulations without the need for adjustments to either the program or the control problem specification.

The solution to the optimal control problem designated by (2.31) to $(2.40)$ can be succinctly summarised as follows. Firstly, $F_{t}$ and $\mathrm{H}_{t}$ are solved backwards in time from $t=T$ to $t=1$. The boundary conditions (2.38) - (2.40) are initially used to compute $\mathrm{F}_{\mathrm{T}}$ and $\mathrm{H}_{\mathrm{T}} \cdot \mathrm{H}_{\mathrm{T}-1}$ can then be computed by substituting $\mathrm{F}_{\mathrm{T}}$ and $\mathrm{H}_{\mathrm{T}}$ into (2.32) and (2.34). This recursive procedure continues until all time periods in the planning period have been accounted for. The resulting $(T k \times n)$ and $(T n \times k)$ matrices are then stored. Secondly, $h_{t}$ and $f_{t}$ are recursively computed backwards in time in the same manner as $F_{t}$ and $H_{t}$ and the resulting ( $T n \times 1$ ) and (Tk $\left.x I\right)$ vectors are stored. When the first two steps have been completed the optimal control (2.31) can be computed from $t=1$ to $t=T$. At each stage of the computation the previous period's optimal state variables are fed 
back into (2.31) to obtain the current optimal control. The only exception is the first period when the initial condition $\mathrm{x}_{0}=\psi$ is used. Finally, after all iterations have taken place, the optimal expected cost for the entire planning period can be calculated using (2.1). A number of observations on the solution procedure are relevant. Firstly, it can be seen immediately from (2.32) that the matrix to be inverted, $\left(C^{\prime} H_{t} C+R_{t}\right)$ is of order $k$, that is, of order equal to the number of control variables. This makes the solution procedure very efficient as in general only small matrices will be required for inversion. The advantage of this is best seen in light of the fact that a twenty period problem would require twenty separate matrix inversions. The efficiency in terms of computer time and computer time and computation tractability is best seen in light of a fixed target problem which for a modest problem of four targets and two controls would require a $(40 \times 40)$ matrix inversion (see below). Another point of some importance is the amount of computer storage required to overcome any potential problems of the "curse of dimensionality" from which the dynamic programming procedure is apt to suffer. $F_{t}$ and $H_{t}$ will be of size $(k \times n)$ and $(\mathrm{n} \times \mathrm{n})$ respectively. Solving for these matrices backwards in time would result in the need to store $2 \mathrm{~T}$ matrices. Even for relatively small problems the storage requirements may be extremely large. Consider a problem which includes five control variables and thirty state variables. It will be of size $(5 \times 30)$ and twenty matrices of size (30 x 30). These forty matrices are equivalent to storing two matrices of size (100 × 30) and $(600 \times 30)$ respectively. The vectors $f_{t}$ and $h_{t}$ must also be stored during computation along with the system matrices $\mathrm{A}$ and $\mathrm{C}$ and the vector $b_{t}$ which would presumably be computed by the program and thus would entail a further data input consisting of the matrix $D$ and the values of all exogenous variables for each time period. One simple method for reducing 
the size of the data to be stored would be to slightly modify the recursive solution procedure set out above such that at each time period $f_{t}$ is calculated immediately from $H_{t}$ thus eliminating the cumulative storage of $\mathrm{H}_{t}$ over the entire planning period. It can be seen from (2.34) that $\mathrm{H}_{t}$ is required to compute $\mathrm{H}_{t-1}$. therefore at any time period of the planning horizon, only two matrices specified by (2.34) will need to be stored on the computer. This will considerably reduce the overall storage requirements and will allow problems of large state dimensions to be efficiently computed on modest computing facilities. The problem of state dimensions is of considerable important as a large model with a considerable number of high order lagged variables could result in a state system as large as $(1000 \times 1000)$ say. In this type of circumstance, the inclusion of the controls as a separate component of the current controls from the state vecotr will substantially reduce the state dimensions as opposed to the Chow formulation. Indeed, the need to convert a model to an equivalent state space realisation (see Chapter Four) may make the solution so prohibitive in terms of size that it may be necessary to turn to the Theil-Tinbergen framework used by Friedman (1975a) which we have seen is in fact equivalent to the linear/quadratic solution, but is subject to its own problem of implementation.

Another issue, related both to the theory of optimal control and the computation of optimal controls, is the question of the existence and uniqueness of optimal economic policies given a particular model. The applied stabilisation literature has almost invariably ignored such questions as has the theoretical economic literature with a few notable exceptions. For example, Turnovsky (1974), (1977b) briefly addresses such issues while a rigorous analysis has recently appeared in the work of Garbade (1976) and Preston (1977). As we shall see below, the question 
of existence and uniqueness is of prime concern in the fixed target framework for stabilisation and there exists similar parallels between the existence and uniqueness criteria for the linear/quadratic and dynamic fixed targets approaches. In many instances it would be difficult to ascertain the existence and uniqueness of an optimal policy before computation is attempted, especially if the model is particularly large and complex. Unfortunately, the problem of existence and uniqueness failures may not be directly transmitted to the applied researcher in the process of computation. For example, in the linear/quadratic framework outlined above, the problem resolves around the existence of the inverse $\left(C^{\prime} H_{t} C+R_{t}\right)^{-1}$. The computer programs which utilise conventional Gauss-Newton inverse sub-routines which do not contain any option for examining the rank of the matrix of concern can in fact produce solutions even when the matrix does not have the desired rank. The results are of course nonsensical but in the absence of any knowledge about the appropriate inverse it is difficult to ascertain whether the result originates from a programming error or a data input error or a failure of existence or uniqueness. It would be highly desirable then to have certain criteria available by which the existence of the inverse $\left(C^{\prime} H_{t} C+R_{t}\right)$ could be established before beginning the computation. A set of existence and uniqueness conditions can be summarised as follows. 4 - First of all consider the case where there are zero control costs, $R_{t}=0$. In this case the matrix of concern reduces to $\left(\mathrm{C}^{\prime} \mathrm{H}_{t} \mathrm{C}\right)^{-1}$. Obviously, a sufficient condition for the inverse to exist is that $\left(\mathrm{C}^{\prime} \mathrm{H}_{t} \mathrm{C}\right)$ has full rank before computation. A set of necessary conditions can be obtained as follows. The sylvester rank inequality theorem states that for two matrices $B^{*}$ and $\bar{B}$, the following will hold, $\rho\left[B^{*} \bar{B}\right] \leq \operatorname{Min}\left[\rho\left[B^{*}\right]\right.$, $\rho[\bar{B}]\}$. Applying this to $\left(\mathrm{C}^{\prime} \mathrm{H}_{t} \mathrm{C}\right)$ it is clear that for $\mathrm{C}^{\prime} \mathrm{H}_{t} \mathrm{C}$ to have full rank it is necessary for $\rho[\mathrm{C}]=\mathrm{k}$ and $\rho[\mathrm{H}] \geq \mathrm{k}$. This condition does not ensure sufficiency however, but it does have the convenient structure of 
requiring the number of targets to be at least equal to the number of instruments. The existence of the inverse will depend on how the instruments affect the particular targets of concern. Note that if at least one lag occurs in all instruments then the necessary conditions are satisfied trivially. Recall that $\mathrm{C}$ is a matrix of size ( $\mathrm{n} \times \mathrm{k}$ ). The rank condition outlined above merely ensures that each instrument has an independent impact on the state vector and does not imply any conditions in relation to particular targets which are a subset of the state vector. For example, we could choose targets (assume less instruments) which do not constitute a set of independent targets, that is, in the reduced form of the structural model the instrument multipliers for the targets will be linear combinations. Under these conditions the necessary conditions could still hold as it could be possible to find a submatrix of $c$, depending on the size of the state (we would require $n>m$ ) which has rank equal to two but the inverse would not exist due to the choice of targets. Consider further the special case where $k=m$, that is, the number of targets equals the number of instruments. Provided the targets are strictly independent then the inverse $\left(\mathrm{C}^{\prime} \mathrm{H}_{t} \mathrm{C}\right)^{-1}$ will exist and the resulting policy will be unique and in addition it is a simple matter to detect the existence and uniqueness before computation provided the reduced form is available. Now consider a situation in which $R_{t}=0$ and $m<k$. It is argued that this type of cost function specification is restrictive as the policy-maker has more instruments than is necessary and greater system control can easily be obtained by adding additional independent targets until $\mathrm{k} \leq \mathrm{m}$. However, the case remains of some interest as it is likely that the policy planner will in some circumstances, direct an apparent surfeit of instruments towards a particular target or targets. For example, directing the S.R.D. ratio and open market operations twoards a money supply target. First of 
all, observe that if $\mathrm{k}>\mathrm{m}$ then from the sylvester rank equality, $\left(\mathrm{C}^{\prime} \mathrm{H}_{t} \mathrm{C}\right)$ will not have full rank but policy will still exist but will no longer be unique due to the inverse failure. This problem can be overcome by resorting to the use of a generalised inverse. Alternatively, the assumption of zero control costs can be dropped and by specifying $R_{t}$ to be strictly positive definite (denoted by $\left.R_{t}>0\right)$, policy uniqueness can be established. Indeed, the appropriate specification of $R_{t}>0$ will lead to policy uniqueness even when $\mathrm{k} \leq \mathrm{m}$ and $\mathrm{C}^{\prime} \mathrm{H}_{t} \mathrm{C}$ is singular. This last condition leads to the interesting situation where solutions can be obtained when some targets are not independent. In general, we can obtain sufficient conditions for policy uniqueness by specifying $R_{t}>0$ (usually only positive elements along the diagonal) or $H_{t}$ positive definite with $\rho(C)=k$ and $R_{t} \geq 0$. The proposition follows directly from the recursive nature of the solution for $H_{t}$ and $u_{t}^{*}$, and like the sufficient condition $R_{t}>0$, allows for policy existence and uniqueness when $\mathrm{m}>\mathrm{k}$ (more targets than instruments). It is clear that if we wish to obtain sufficiency without $R_{t}>0$ then the structure of the model, the framework and hence the feedback matrices must be conducive to establishing $\mathrm{H}_{t}>0$. It should be noted that the strong conditions on $\mathrm{H}_{t}$ or $\mathrm{R}_{t}$ are not necessary for policy uniqueness except when $\mathrm{k}>\mathrm{m}$ (see above). Indeed, $\left(\mathrm{C}^{\prime} \mathrm{H}_{t} \mathrm{C}+\mathrm{k}_{t}\right)^{-1}$ can exist as we have seen when $\mathrm{H}_{t}>0$ and $\mathrm{C}^{\prime} \mathrm{H}_{t} \mathrm{C}$ is singular and even given $\mathrm{H}_{t}>0, \mathrm{C}^{\prime} \mathrm{H}_{t} \mathrm{C}$ may be singular if $\rho[C] \neq k .{ }^{5}$ While various criteria can be established for policy uniqueness, it should be noted that the structure of the model will play an important role in this question at the computation level as it does in the dynamic fixed target problem. However, as we shall see below, the existence and uniqueness criteria for the latter framework are of a more simple nature than the conditions for the linear/quadratic solution. 
OPTIMAL STABILISATION POLICY: SOME PRACTICAL CONSIDERATIONS

Before proceeding any further with developing the framework of this study, it is convenient to briefly discuss some of the practical considerations associated with implementing optimal stabilisation policies. A discussion of this aspect is warranted in light of the attitude of many economists working in the optimal stabilisation field. In particular, many researchers fail to discuss the feasibility of actually using optimal control as a tool for stabilisation. Leaving aside the traditional criticisms of the technique on the grounds of misspecified models, uncertainty about the structural parameters and lags, and our ability to adequately forecast uncontrollable exogenous variables (more of this later) I will focus on the implementation of policy.

Consider the equation relation to the optimal control to the lagged state, $u_{t}^{*}=F x_{t-1}+f_{t}$. Given that some components of the lagged state vector are true first order lagged variables then it is almost certain that the type of dynamic policy response described by the feedback relationship above will not be applicable in practice. Firstly, even if the true exact value of $x_{t-1}$. were known at the beginning of the current unit time period (period $t$ as opposed to the entire planning period), the policy decision - and transmission process of governments and the bureaucracy would prevent the desired value of $u_{t}^{*}$ from being achieved in that period. The feedback relationship implies that as data about the past state does not become available until the end of the current time period, which of course is the beginning of the next time period, the implementation of policy is instantaneous! If the assumption about some components of the past state consists of say lags greater than two or three periods (converted of course to equivalent one period lag structures - see chapter Four) then it is possible that policy may be formulated before the required time period 
with sufficient time to implement it. However, even the presence of long lags does pose some difficulties as the new variables defined to replace those with lags greater than one period will also play an important role in the feedback matrix $F_{t}$ and contribute to the formulation of $u_{t}^{*}$. This will become apparent in the ensuing applied discussion in later chapters. One possible solution to the problem would be to make the planning period less than say the longest lag to prevent some auxiliary variables from taking on values in the feedback matrix. Once again, the reader is referred to future chapters where feedback matrices for the applied experiments are presented and where it can be seen how auxiliary variables drop out of the feedback matrices as the planning period approaches its terminal time. The important point to be made here is that policy planners would need to plan some time in advance in order to ensure that their desired policies do in fact become realised at time $t$. The need for forward planning refers mainly to government spending as instruments such as tax rates and open market operations can be carried out almost instantaneously in terms of say a quarterly model. A further need for forward planning in relation to government spending arises from a need to calculate the government's budget position for the entire year. In a quarterly model this would entail planning optimally at least four quarters in advance. The compilation of the budget and the allocation of funds to various factors also requires time resulting in a need for perhaps a total of six quarters advance time before the optimal policy can be implemented. At first sight it could be argued that all policy lags of the system (not just impact lags) could be explicitly modelled. While this is feasible in theoretical optimal stabilisation work, for example Preston (1975) and Turnovsky (1977a) its extension to an applied framework is highly dubious due to the great variability from time period to time period in the lags involved which can 
occur through bureaucratic, institutional and political reasons. 6

The need for forward planning also necessitates using forecasts of the state vector $x_{t-1}$ to obtain $u_{t}^{*}$ and thus the optimal control will not be able to adjust for additive disturbances with the result that the implemented $u_{t}$ will be sub-optimal. Even if the shocks are extremely small, the model may not be able to adequately forecast the future and the policy-maker will be severely limited in his ability to take account of uncertainty. Whether or not ignorance of future shocks and poor forecasts will pose serious problems, especially when fine tuning is desired, depends on the degree of sub-optimality. Even if instantaneous policy adjustment can be carried out there still remains the problem of gaining good information about the immediate past state. It may be necessary to derive the optimal control before the final figures for say income, have been formulated for a particular quarter. Initial estimates can contain "noise" elements which may obscure the "true" value considerably. The problem of uncertainty about the immediate past state whether generated by a need for forward planning or by an inability to correctly observe the state at the appropriate time could severely limit the application of optimal techniques as a knowledge of $x_{t-1}$ is required in each time period. As we shall see below, the computation of fixed target solutions only relies on a single estimate of the initial condtions and hence will suffer less from errors in the state. Nonetheless for real world applications it would most likely be necessary to plan in advance even within the fixed target framework. While adaptive learning techniques may be useful when policy implementation is instantaneous, they will be extremely limited if policy decisions need to be made well in advance of the time they are to be implemented. In any case, the experimental use of learning procedures has not, in many cases, significantly improved the 
performance of optimal stabilisation, for example the work by Abel (1975), Macrae (1972), and Walsh and Cruz (1976).

\section{THE DYNAMIC FIXED TARGET FRAMEWORK}

In this section the dynamic fixed target framework for stabilisation will be developed and discussed with the discussion drawing heavily on the recent work of Preston and Sieper (1977). The development of a fixed target framework relies heavily on the control theory concept of controllability, a concept which has, along with existence and uniqueness, been largely ignored in the economic literature - particularly in the applied literature. The concept of controllability forms a fundamental component of control theory as applied in the sciences and stems from the work of Kalman (1960a) (1960b). The notion of controllability along with the concept of observability provides necessary conditions and in specific cases, sufficient conditions, for a control problem to possess a solution. Observability need not concern us here. A useful reference which employs the concept of observability in the development of existence criteria is the work by Preston (1977) mentioned above. When talking about controllability it is necessary to distinguish between two basic types - state controllability and output controllability. Basically, state controllability is a property of a given system which ensures that any given initial state can be transferred to any previously specified terminal state in a finite time span. Conversely, output controllability refers to the achievement of any desired or target terminal target vector in finite time starting from some arbitrary initial state. The development of the linear/quadratic solution outlined above was in terms of the state vector as opposed to an output vector (in economic terms outputs refer to the endogenous variables of a system). 
The framework developed here will be in terms of outputs and the subsequent controllability criteria developed will refer to the achievement of specific outputs/targets which form a subset of the state space vector of the system.

We begin the discussion by looking briefly at the static Tinbergen analysis of economic policy and then extending this to a dynamic framework. A general static model can be represented by

$$
\overline{\mathrm{A}} \mathrm{y}=\overline{\mathrm{C}} \mathrm{u}+\overline{\mathrm{D}} z
$$

which can be rewritten in reduced form as

$$
\mathrm{y}=\overline{\mathrm{A}}-1 \overline{\mathrm{C}} \mathrm{u}+\mathrm{A}^{-1} \overline{\mathrm{D}} \mathrm{z}
$$

or

$$
y=C u+D z
$$

which is in the notation set out at the beginning of this chapter with the addition that $\mathrm{y}$ replaces $\mathrm{x}$ and refers to the targets outputs of the system. That is, it is assumed that all non-target variables have been explicitly ignored. $y$ is an $(m \times 1)$ vector, $c$ is an $(m \times k)$ matrix, $u$ is a $(k \times 1)$ vector, $D$ is an $(m \times j)$ matrix and $z$ is a $(j \times 1)$ vector where $m$ equals the number of outputs/targets, $k$ equals the number of instruments and $j$ equals the number of exogenous variables. From (2.46) we can obtain a number of basic propositions concerning the static achievement of targets. Firstly, if $\rho[C]=k=m$ then it follows that there exists a policy set which is unique and allows the exact achievement of the target vector $y$ and is given by

$$
u=C^{-1} y-C^{-1} D z
$$

Furthermore, if $\rho[C]=m<k$. then policy will exist but will not be unique and if $\rho[\mathrm{C}]=\mathrm{k}<\mathrm{m}$ then policy will not exist. The above 
conditions simply state the familiar Tinbergen static counting rules along with a sufficient rank condition. That is, a necessary condition for policy existence is that the number of instruments must equal or be greater than the number of targets. A sufficient condition for existence and uniqueness is given by the matrix of instrument multipliers having full rank equal to both the number of instruments and targets. If the number of targets is less than the number of instruments then policy will still exist but will no longer be unique. The observations are standard but it is interesting to compare them with the existence and uniqueness criteria for the dynamic linear/quadratic problem. Recall that in the linear/quadratic framework it was possible to obtain a set of policies even if the number of targets exceeded the number of instruments and as such is a more flexible approach than the static Tinbergen approach. The solution (2.47) implies free adjustment of all instruments while it was recognised that to obtain uniqueness in the linear/quadratic (static and dynamic) case it may be necessary to weight the controls. The weighting of controls need not be a hindrance as the weights can be specified to be very small in relation to the state weights and thus approximate zero. It may not seem appropriate to compare the static and dynamic cases but it is an easy matter to establish similar conditions for the static linear/quadratic case which again is more flexible in its application in relation to obtaining policies when the number of targets exceeds the number of instruments or even if an instrument rank failure exists. The disadvantage of a flexible target approach may be that if $\mathrm{m}>\mathrm{k}$ then targets will be traded-off uniess all targets are perfectly consistent. As we shall see below, the introduction of dynamics into the system will enable the fixed target approach to take on a similar degree of flexibility to the Iinear/quadratic approach although the 
basic solution techniques retain the same simple general form as the static case given by (2.47)

Assuming, time invariant coefficient matrices and perfect forecasting of the uncontrollable exogenous variables, the dynamic fixed target framework can be developed as follows. (2.44) can be expressed in dynamic form as

$$
\bar{A}(L) y_{t}=\bar{C}(L) u_{t}+\bar{D}(L) z_{t}
$$

where $L$ refers to the delay operator of the form $L \mathrm{~V}_{t}=\mathrm{x}_{t-\mathrm{v}}$ and consequently $\bar{Z}(L), \bar{C}(L)$ and $\bar{D}(L)$ are polynomial matrix coefficients which are defined as

$$
\begin{aligned}
& \bar{A}(L)=\sum_{i=0}^{e} \bar{A}_{i} L_{i} \\
& \bar{C}(L) \equiv \sum_{i=0}^{f} \bar{C}_{i} L^{i}
\end{aligned}
$$

and

$$
\bar{D}(L) \equiv \sum_{i=0}^{g} \bar{D}_{i} L^{i}
$$

where e,f and $g$ represent the longest lag of the targets, controls and exogenous variables respectively. Note that imbedded in the dynamic structure of (2.48) there is a static steady state model given by

$$
\overline{\mathrm{A}}(1) \mathrm{y}=\overline{\mathrm{C}}(1) \mathrm{u}+\overline{\mathrm{D}}(1) \mathrm{z}
$$

which of course is identical to (2.44). Even if the steady state can be achieved in a fairly rapid time period, it will in most cases not be instantaneous and thus the policy-maker will need to anticipate his target if he wishes to achieve a stationary target, $y$. Following the notation of Preston and Sieper we shall denote a dynamic policy model (2.48) with matrix $\vec{C}(1)$ which satisfies the condition that $\rho[\bar{C}(1)]=m$ 
as weakly-Tinbergen. Conversely, if we consider the reduced form (2.48) for simplicity, a situation in which the impact multiplier matrix of control coefficients has rank equal to $\mathrm{m}$, shall be denoted strongly Tinbergen. In a dynamic strongly-Tinbergen world we simply treat all lagged controls, targets and exogenous variables as given predetermined variables and solve the policy problem as we would for the static case. That is, given the same number of instruments and targets in a dynamic world and provided the targets constitute independent targets so the matrix of reduced form instrument multipliers has sufficient rank, it is possible for the policy planner to hit all his targets without anticipation through the concurrent manipulation of available instruments. We shall also examine the controllability of systems designated as non-Tinbergen where $\mathrm{k}<\mathrm{m}$ but for which existence criteria can be formulated in contrast to the static case.

To develop a dynamic fixed target framework for stabilisation two important concepts must be considered. The first is the final form of an econometric model and the second is the state space form of a model. The final form of (2.48) can be written as (provided the targets are naturally stable)

$$
y_{t}=\bar{A}^{-1}(L) \bar{C}(L) u_{t}+\bar{A}^{-I} \bar{D}(L) z_{t}
$$

and is important in terms of the dynamic multipliers associated with it. Secondly, the state space realisation of a structural economic model such as (2.48) can be written as

$$
\begin{aligned}
& x_{t+1}=\hat{F} x_{t}+\hat{G} u_{t}+E z_{t} \\
& y_{t}=P x_{t}+\hat{S} u_{t}+M z_{t}
\end{aligned}
$$


where $\hat{F}$ and $\hat{G}$ are written with a hat to avoid confusion with the notation used in the derivation of the control laws and where $x_{t+1}$ represents the state vector as before. The state space realisation. given by $(2.52)$ is the usual formulation found in the non-economic control literature and differs from the simple reduced form state space used at the beginning of the chapter. To maintain consistency with the literature (2.52) will be used to derive controllability conditions for the fixed target approach although it should be pointed out that $(2.52)$ is equivalent to the first order realisation employed in the linear/ quadratic solution. A comparison and discussion of the two techniques is given in Chapter Four. There are two important reasons for using a state space realisation in this context. Firstly, analytically, it is easier to investigate the dynamics of the problem within the context of a first order system such as (2.52) and secondly, related to the first issue is that a potentially large number of structural form polynomial structural coefficients matrices, $\bar{A}(L), \bar{C}(L)$ and $\bar{D}(L)$ can be replaced by constant coefficient matrices $\hat{F}, \hat{G}, E, P, \hat{S}$ and $M$. However, the use of (2.52) also reduces our economic insight into any analysis which is pursued. This is contrary to the form used at the beginning of the chapter where the original reduced form structure is maintained throughout. The problem of insight and intexpretation can be overcome in the specific case of the dynamic fixed target framework analysed here as the problem essentially reduces to the manipulation of dynamic multipliers - a concept familiar to economists.

To establish the solution of the problem in terms of the dynamic multipliers of (2.48), the final form and state space form can be utilised. ${ }^{8}$ Using the delay operator in the state dynamics of the system (2.52a) gives 


$$
x_{t}=[I-L F]^{-1}\left(\hat{G L u} u_{t}+E L z_{t}\right)
$$

Substituting (2.53) into the target mapping (2.52b) gives

$$
y_{t}=\left[\hat{S}+P(I-L F)^{-1} \hat{G L}\right] u_{t}+\left[M+P(I-L F)^{-1} z_{t}\right.
$$

Using (2.54) and the expression for the final form (2.51) we have the following identities

$$
\begin{aligned}
& \overline{\mathrm{A}}^{-1}(L) \bar{C}(L) \equiv \pi(L) \equiv \hat{S}+P[I-L F]^{-1} \hat{G L} \\
& \bar{A}^{-1}(L) D(L) \equiv \Gamma(L) \equiv M+P[I-L F]^{-1} E L
\end{aligned}
$$

Power series expansions for the last two identities in (2.55a) yields

$$
\sum_{i=0}^{\infty} \pi_{i} L^{i} \equiv \hat{S}+P\left(\sum_{i=1}^{\infty} F^{i-1} L^{i}\right) \hat{G}
$$

which immediately gives us a relationship between the dynamic instrument multipliers of the system in terms of the state space constant matrices. In particular we have that

$$
\pi_{0} \equiv \hat{\mathrm{S}}, \pi_{1} \equiv \mathrm{PG}, \pi_{2} \equiv \mathrm{P} \hat{\mathrm{FG}}, \ldots . \pi_{\mathrm{T}} \equiv \mathrm{P} \hat{\mathrm{F}}^{\mathrm{T}-\mathrm{I}} \hat{\mathrm{G}} \ldots
$$

where $\pi_{i}$ represents the dynamic instrument multipliers and the subscript refers to the period impact of the multiplier.

Having developed the appropriate concepts we are now able to derive solutions for two types of fixed target policy. The first type of problem to be considered is the achievement of a target point objective which is, as we shall see below, a pre-requisite for target path achievement which is a discrete time extension of the continuous time. Tinbergen framework developed in Preston (1974). The achievement of a target point objective incorporates the concept of a policy lead, denoted by s, the rationale for which will become much clearer as the analysis proceeds. The problem then, is to find 
a policy sequence (assuming existence) beginning at time $t=0$ which consists of the set $u_{0}, u_{1}, \ldots u_{s}$ which is able to steer a set of outputs from some arbitrary initial position, $\mathrm{y}_{0^{\prime}}$ such that desired targets for the outputs, $\bar{y}_{S^{\prime}}$, are achieved at some period in the future, $s$, where $s$ also denotes the length of the policy lead or degree of anticipation required or desired. Basically we are asking if it is possible to adjust our instruments over time in order to achieve a desired set of target values. Note that as yet nothing has been said about the number of targets or the number of instruments.

The solution to the problem is obtained by initially performing $s$ iterations of the state dynamics $(2.52 a)$ to produce the following equations:

$$
\begin{aligned}
& x_{1}=\hat{F} x_{0}+\hat{G} u_{0}+E z_{0}
\end{aligned}
$$

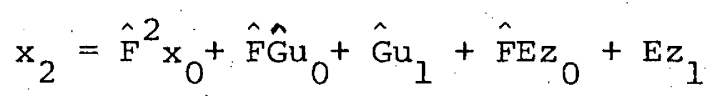

$$
\begin{aligned}
& \vdots \\
& x_{s}=\hat{F}^{s} x_{0}+\left[\hat{G F G} \ldots \hat{F}^{s-1} \hat{G}\right]\left[\begin{array}{c}
u_{s-1} \\
u_{s-2} \\
\vdots \\
u_{0}
\end{array}\right]+\left[\hat{F F E} \ldots \hat{F}^{s-1} E\right]\left[\begin{array}{c}
z_{s-1} \\
z_{s-2} \\
\vdots \\
z_{0}
\end{array}\right]
\end{aligned}
$$

Similarly, the sth iteration of (2.52a) yields an expression for the target point $y_{s}$ given by 


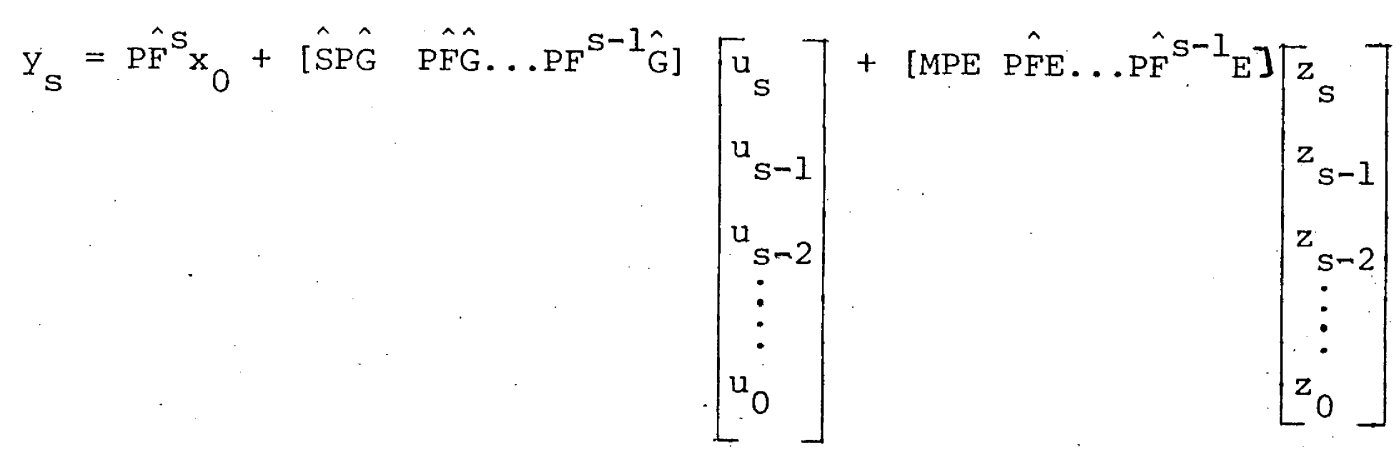

which can be written compactly as

$$
\mathrm{y}_{\mathrm{s}}=\mathrm{P} \hat{\mathrm{F}}^{\mathrm{S}} \mathrm{x}_{0}+\mathrm{B}_{\mathrm{S}} \mathrm{u}_{\mathrm{s}}+\hat{\mathrm{B}} \mathrm{z}_{\mathrm{S}}
$$

where $\mathrm{y}_{\mathrm{S}}$ is an (mxl) vector, $\mathrm{B}_{\mathrm{S}}$ is an $(\mathrm{mx}[\mathrm{s}+1] \mathrm{k})$ matrix, $\mathrm{u}_{\mathrm{s}}$ is an $([s+1] j \times l)$ vector. The target point objective depends on the initial conditions $\mathrm{x}_{0}$, the uncontrollable exogenous variables which are assumed to be known exactly in advance and the discretionary policy sequence given by the vector $u_{s}$. Assuming that we know $x_{0}$ and $z_{s}$ ' then it only remains to determine $u_{s}$ and provided the existence of a discretionary policy sequence can be established, the appropriate $u_{s}$ can be obtained from solving the linear system $(2.60)$. It is clear from (2.60) that a sufficient condition for the existence of a solution to the target point problem is that the matrix $B$ has rank equal to $m$. If $\rho[B]=m$ then the target point problem has a solution

$$
u_{S}=B_{S}^{-1} \bar{y}_{S}-B^{-1} \hat{p F} \hat{x}_{0}-\hat{B}^{-1} B_{S} 9
$$

where $\bar{y}$ designates some desired target level. The controllability condition can be expressed more formally as

$$
\rho\left[\mathrm{B}_{\mathrm{S}}\right]=\rho\left[\hat{\mathrm{S}} \mathrm{P} \hat{\mathrm{G}} \mathrm{P} \hat{\mathrm{F}} \hat{\mathrm{G}} \ldots \mathrm{P} \hat{\mathrm{F}}^{\mathrm{S}-1} \hat{\mathrm{G}}\right]=\mathrm{m}
$$

Note also that the relationship between the state space realisation and the dynamic multipliers of the system yields an equivalent controllability condition 


$$
\rho\left[B_{S}\right]=\rho\left[\pi_{0} \pi_{1} \pi_{2} \cdots \pi_{S}\right]=m .
$$

Also note that the solution to the dynamic target point objective problem given by (2.61) is of an identical procedure to the solution of the static Tinbergen problem given by (2.47). The relationship between $\pi_{i}$ and the state space matrices makes the solution given by (2.61) very simple. The dynamic instrument and exogenous variable multipliers are substituted into (2.61) and the problem is solved by carrying out a simple matrix inversion and matrix multiplication. Note that the dimensions of the system require that the matrix to be inverted is of order $m$, the number of targets. For large target configurations obtaining the inverse could lead to numerical problems. This is in contrast to the linear/quadratic solution where the matrix to be inverted is always of order $k$. Therefore even if there are 100 targets and twenty instruments the matrix to be inverted will only be of order 20 while in the fixed target point control problem the matrix would be of order 100. The target point problem also allows the policy planner to hit his desired targets when $\mathrm{k}<\mathrm{m}$ (provided the existence criterion is fulfilled). By redefining the notion of instruments within a dynamic context as considering the same instrument at different points in time as a distinct instrument or time indexed instrument we have a dynamic counterpart of the static Tinbergen counting rule, that is, the number of time indexed instruments must be greater than or equal to the number of targets. The counting rule is given by

$$
s \geq \frac{m}{k-1}
$$

or $" k " \equiv k(s+1) \geq m$

where " $k$ " indicates the number of time indexed instruments. 10 In this sense controllability conditions for the static and dynamic cases are basically the same. Also, a dynamic formulation of policy allows the 
policy-maker to hit more targets than he has "true" or non-time indexed instruments which is similar to the linear/quadratic situation where it is possible to have policy existence but of course targets will be tradedoff rather than exactly hit unless the targets are specified to be perfectly consistent. The problem of more instruments than targets, whether true instruments or time indexed instruments, still poses problems of non-uniqueness. This problem can be overcome by simply setting any slack instruments to any pre-designated levels just leaving enough instruments to satisfy the controllability conditions. The pre-set slack instruments can then be treated as exogenous variables. In many cases the impact instrument multiplier $\hat{S}=\pi_{0}$ will have sufficient rank to satisfy the controllability condition. Under these conditions the policy-maker can hit his targets instantaneously without resorting to the need for a policy lead. Alternatively, the policy-maker can do as he pleases for the periods leading up to the time period to achieve his desired objective. The example of sufficient rank in $\pi_{0}$ of course corresponds to the strongly-Tinbergen condition outlined above.

Just as there is a lower bound to the policy leads, there is also an upper bound. That is, there is some point where the future can no longer be affected by adding additional time indexed instruments. This result follows from the Cayley-Hamilton theorem which when applied to the problem presented here tells us that $\hat{\mathrm{F}}^{\mathrm{S}}$ is a linear combination of the first $n$ powers of the $n \times n \operatorname{matrix} \hat{F}$, for all $s \geq n$. Hence the extension of the policy lead $\mathrm{s}$ beyond the state dimension $\mathrm{n}$ will not add any additional linearly independent columns to $\mathrm{B}_{\mathrm{n}}$ (see 2.60). It follows that the policy lead has an upper bound

$$
\mathrm{s} \leq \mathrm{n}
$$

and must lie between the bounds 


$$
\frac{\mathrm{m}}{\mathrm{k}-1} \leq \mathrm{s} \leq \mathrm{n}
$$

Having developed criteria for the exact achievement of a target point objective and briefly noted the similarities with a corresponding static analysis, we now turn to the more interesting case of an exact target path within a dynamic context. Once again we shall derive a dynamic counterpart to the static Tinbergen counting rule. As with the target point problem the policy lead $\mathrm{s}$ will be of prime importance. To make the analysis clearer the concept of a planning period in relation to a fixed target problem will be made more explicit. The planning period consists of two basic components the familiar policy lead $s$ and difference between the policy lead or anticipation period and the total planning period. The target path problem then, is to choose a discretionary policy sequence over the entire planning period which will enable the policy planner to realise his target objectives over a subset of the planning period. The concept is best illustrated diagrammatically by utilising the diagram in Preston and Sieper (1977 p.221).

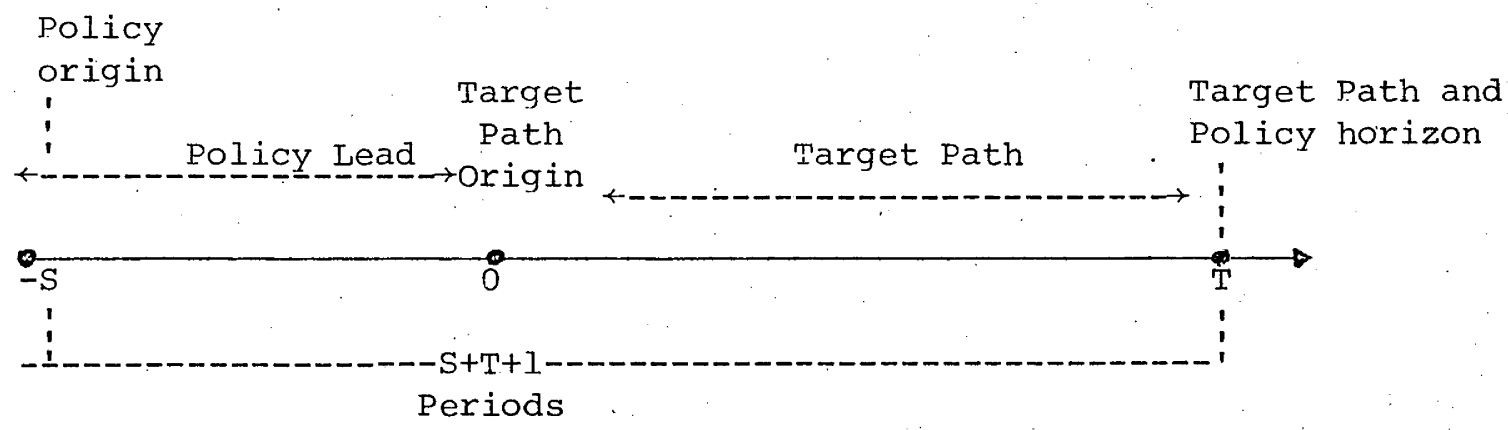

Policy Anticipation Framework

Figure 1 .

It can be seen from Fig. I that the target point objective discussed above is a special case of the target path problem with the target point being achieved at period 0 with lead s, a total policy interval of 
$s+1$ periods. The introduction of a target path implies that policymakers wish to hit and maintain pre-selected levels of their targets for some specific length of time. The target path objective can be viewed as a series of point objectives and it is clear that if our target path origin is to begin at 0 in Fig.l then quite clearly the policy model, given the desired targets and respective instrument multipliers, must be point controllable at the target path origin. Thus we wish to ascertain the existence of a policy sequence

$$
u_{S, T} \equiv\left(u_{-S}, \ldots, u_{0}, \ldots u_{T}\right)
$$

given arbitrary initial conditions and arbitrary values of the exogenous variables which can anticipate our target path objective such that the target path objective

$$
\mathrm{y}_{\mathrm{T}}^{*} \equiv\left(\mathrm{y}_{0}, \cdots, \mathrm{y}_{\mathrm{T}}\right)
$$

can be achieved. Note that the components of $\mathrm{y}_{\mathrm{T}}^{*} ; \mathrm{y}_{0}, \mathrm{y}_{1}$ etc. are in fact vectors consisting of the number of targets to be achieved in each time period. Thus if the policy planner wishes to achieve $m$ targets in each time period of the target path, then the total number of targets to be aimed for will be $m(T+1)$.

Firstly, consider the situation in which the instrument impact multiplier is of full rank

$$
\rho[\hat{S}]=\rho\left[\pi_{0}\right]=m \leq k
$$

In this case we have a strongly-Tinbergen situation and a special extension of the target point problem. (2.69) ensures that the policy model with structural coefficients $\bar{A}(L), \bar{C}(L)$ and $\bar{D}(L)$ will be both target point controllable and target path controllable with zero policy lead. This is of course equivalent to the static Tinbergen situation 
in which the number of targets must equal the number of instruments. The dynamic case can be extended however, to include situations in which the impact instrument multipliers have zero rank and $\rho\left[\pi_{1}\right]=m \leq k$. This condition applies where the instruments have no effect on the targets until the next period. Given this situation the policy-maker will need to anticipate his targets by one period but given full rank in $\pi_{1}$ he is still able to keep the system on target for every period as long as the policy is decided upon the period before the target is achieved. A similar argument holds for $\pi_{2}$ etc. For most policy purposes situations will exist in which the number of targets exceeds the number of instruments and $\pi_{0}$ or $\pi_{i}$ will not have sufficient rank to enable the policy-maker to exactly achieve his targets instantaneously by the manipulation of his instruments within the current time period. In this case the policy-maker will need to adjust his instruments over time to anticipate his targets. An expression for the achievement of the target path objective for lead s can be obtained by applying (2.64) for $t=0,1 \ldots$, that is for each element of the target path vector $\mathrm{y}_{\mathrm{T}}^{*} \equiv\left(\mathrm{y}_{0}, \mathrm{y}_{1} \cdots \mathrm{y}_{\mathrm{T}}\right)$, and considering a general situation where the origin becomes $-\mathrm{s}$ and a time span of $\mathrm{T}+\mathrm{s}$ is considered, giving (excluding the exogenous variables for simplicity)

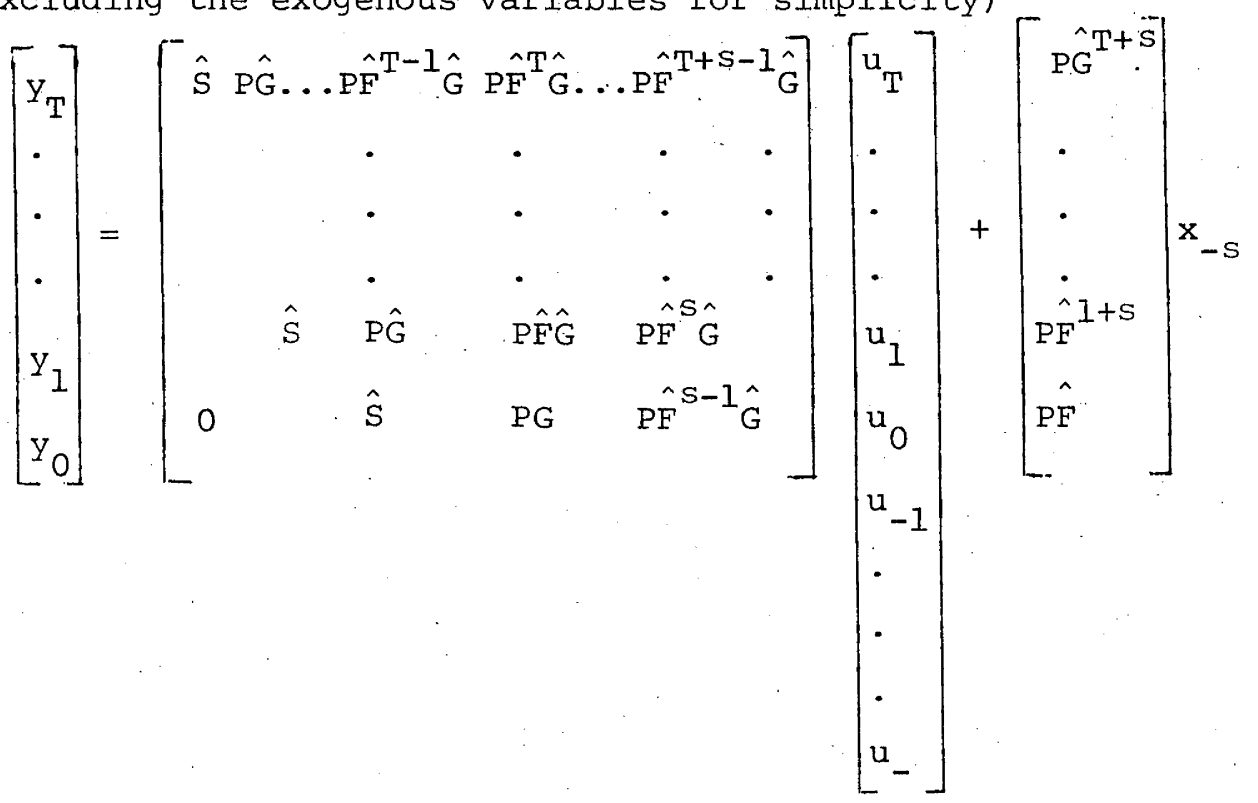


By dropping the time indices from (2.70) and reintroducing the exogenous variables, the stacked matrices and vectors can be written compactly as

$$
\hat{\mathrm{Y}}=\hat{\mathrm{Ru}}+\hat{\mathrm{Dz}}+\hat{\mathrm{Px}}-\mathrm{s}
$$

where the "A" on $y, R, u, D$ and $P$ has been introduced to avoid the repetition of scarce notation. Note that the subscript, $-s$, on the state vector $\mathrm{x}$ refers to the initial conditions at the beginning of the policy lead. (2.71) is, excluding the initial conditions, in the same general form as the static policy model $(2.46)$ and the point controllable framework of $(2.60)$. The dimensions of $(2.71)$ are as follows. $\hat{y}$ is an $(m(T+1)$ vector, $\hat{R}$ is an $(m(T+I) \times k(T+S+I))$ matrix, $\hat{u}$ is a $(k(T+S+1) \times l)$ vector, $\hat{D}$ is an $(m(T+l) x j(T+s+l))$ matrix, $\hat{z}$ is a $(j(T+s \times l) x l)$ vector, $\hat{P}$ is an $(\mathrm{m}(\mathrm{T}+1) \mathrm{xn})$ matrix and $\mathrm{x}$ is of course an $\mathrm{n} \times 1$ vector. The system dimensions and (2.71) immediately gives as a sufficient condition for the existence of $\hat{u}$ which will allow the achievement of the target path $\hat{y}$ from $O$ to $T$ with lead $s$. The linear dynamic model with coefficient matrices $\bar{A}(L), \bar{C}(L)$ and $\bar{D}(L)$ with equivalent state space structure given by the matrices $\hat{F}, \hat{G}, E, P, \hat{S}$ and $M$ is target path controllable with policy lead or anticipation, $s, s \varepsilon(0,1 \ldots)$ and target interval $T+1$ for any arbitrary initial conditions $\mathrm{x}_{-\mathrm{s}}$ and any arbitrary values of the exogenous variables, if and only if, the $(\mathrm{m}(\mathrm{T}+\mathrm{l}) \mathrm{xk}(\mathrm{T}+\mathrm{S}+\mathrm{l})$ ) controllability matrix, $\hat{R}$, satisfies

$$
\rho[\hat{R}]=m(T+1)
$$

where $\hat{\mathrm{R}}$ is of course equal to the matrix of (2.70). Furthermore, from (2.57) and (2.70) we have an equivalent controllability condition 


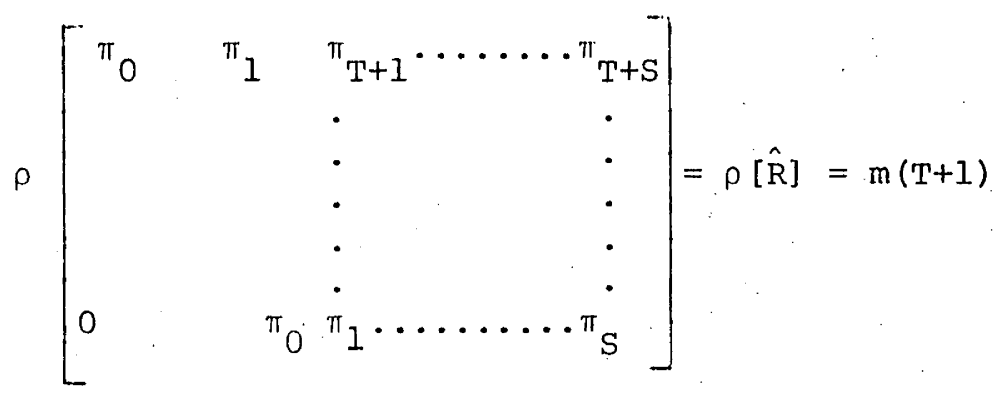

The fulfilment of the sufficient condition for dynamic policy existence allows a solution to the problem $(2.71)$ to be obtained. The solution can be found by using the dynamic instrument multipliers in the controllability matrix and using the dynamic multipliers for the exogenous variables which are related to the state space realisation in the same general way as the instruments, to give an expression for the dynamic values of the instrument

$$
\hat{u}=\hat{R}^{-1} \hat{y}-\hat{R}^{-1} \hat{D} \hat{z}-\hat{R}^{-1} \hat{P}_{-S}
$$

(2.74) is conceptually a very simple problem to solve, certainly more simple than the linear/quadratic approach to dynamic stabilisation. However, there are some very real problems associated with solution of (2.76) in an applied framework as we shall see below and in a later chapter. The sufficient condition if satisfied, enables the policy-maker to exactly achieve his targets over the period $\mathrm{T}+1$ even in a non stronglyTinbergen world where $\mathrm{k}<\mathrm{m}$ so long as the policy-maker is willing to anticipate his target by s periods. The dimensions of the controllability matrix $\hat{R}$ and the rank criterion for sufficiency lead to a dynamic counterpart of Tinbergen's counting rule. Thus, a necessary condition. for dynamic target path achievement is that the number of time indexed instruments must be greater than or equal to the total number of targets over the target path. That is,

$$
" k^{\prime} \equiv k(T+S+1) \geq " m " \equiv m(T+1)
$$

In many instances the column dimension of $\hat{R}, k(T+S+1)$, will be greater than 
the row dimension $m(T+1)$ and the matrix will not be square hence precluding the existence of the regular inverse. That is, if the matrix has full rank then we will have more instruments than are necessary to achieve the desired target configuration. To overcome this problem, time indexed instruments can be dropped from $\hat{R}$ until $\hat{R}$ has dimension $(\mathrm{m}(\mathrm{T}+1) \mathrm{xm}(\mathrm{T}+1))$. The excluded "instruments" can then be assigned predetermined values and treated as exogenous variables although in practice it would be difficult to ascertain at which level to fix the instruments. The numerical values chosen for the "slack" instruments will of course affect the values obtained for $\hat{\mathrm{u}}$ in the solution. While there may be some justifiable reasons for government spending at a pre-determined level, for example social and political reasons, there would appear to be little justification for setting monetary instruments at a particular level for say one period. This would be especially so when the time indexed monetary instrument was dropped from the anticipation period. The sensitivity of fixed target solutions to the dropping of slack instruments and the values assigned to those instruments would be of prime concern in an applied framework and will be a matter for investigation in this study. As with the target point problem, an upper bound on the amount of target path anticipation can be derived from the Cayley-Hamilton theorem. The upper bound for the target path problem is $s=n$, that is; once the level of anticipation equals the number of state variables no further linearly independent time indexed instruments can be found.

Tentative comparisons between the fixed target framework and the linear/quadratic framework have already been made. The most striking difference between the two techniques which has already been alluded to in Chapter One is that in many cases the policy-maker will not be able to achieve all his targets over a planning horizon with a limited amount of 
instruments. Implicit in the linear/quadratic case is a zero policy lead implying that the policy-maker expresses excessive impatience in trying to achieve his targets. The end result is that his targets will be compromised. On the other hand, the fixed target approach allows a policymaker to exactly hit his target within the bounds of the planning horizon as long as he is prepared to wait. Which technique to choose? The linear/ quadratic solution for a particular problem may result in all targets being compromised but the degree of compromise may be small in all periods. The fixed target solution for an identical problem may entail being considerably off target for two thirds of the planning period and only exactly on target for the remaining third of the planning period. The great divergence from targets in the initial stages of the planning period may result in structural shifts as the expectations of the private sector change. The maintenance of a constant structure is crucial for a fixed target approach and while a constant structure has been assumed for the linear/ quadratic case, recent developments in that area have allowed for learning techniques to be incorporated in the stabilisation procedure. In this respect the fixed target approach is more restrictive.

There are also some similarities in the existence criteria of both techniques. Specifically, if the targets chosen are independent and the number of instruments equals the number of targets then the existence of policy in both frameworks is guaranteed. However, in such a strongly-Tinbergen world the Zinear/quadratic solution becomes redundant and the problem can be solved by the computationally simpler fixed target technique. Both stabilisation techniques allow for policy existence when the number of natural targets exceeds the number of natural 
instruments. However, the dynamic Tinbergen solution still requires equality betweeen "targets" and "instruments" in a time indexed sense and hence the policy planner is constrained in relation to the number of targets he can choose given his available instruments and the length of the planning period. In this respect, the optimisation approach is more flexible as the number of time indexed instruments and targets has no part in establishing the existence of policy and the policy planner is free to choose any number of targets relative to instruments and will not be constrained by the length of the planning period. While the optimising policy planner is free to choose many more targets than instruments than may be possible in a fixed target framework, the choice of targets still must be consistent with the computation of $\left(C^{\prime} H_{t} C+R_{t}\right)^{-1}$. While it may not be possible to ascertain if the sufficient conditions for policy existence are satisfied in the linear/quadratic case prior to computation, it is possible in the target point situation when we only need to establish the existence of the inverse of a matrix of order $\mathrm{m}$. If the number of targets is about four then this is a relatively easy exercise - not so however, for the target path problem. Indeed, the size of the matrix to be inverted in the target path situation is one of the drawbacks of the technique.' Consider a planning horizon of twenty periods with four targets and two instruments. Assuming existence, the linear/quadratic solution would require twenty. $(2 \times 2)$ matrix inversions, the target point solution would require one $(4 \times 4)$ inversion while the target path solution would require one $(40 \times 40)$ matrix inversion and in addition, the controllability matrix will contain a large number of zero elements, from (2.74). Typically, the non-zero elements will be very small which could make inversion difficult with the possibility occurring of computer rounding and rounding of the data input creating 
rank deficiencies and Iinear dependence between instruments when in fact none exist. Further insight into this problem can be gained from Chapter Seven.

The relative adjustment of both techniques to uncertainty is also important. As presented here, neither technique is able to adjust to parameter uncertainty although it must be recognised that the optimisation literature has begun to advance techniques to handle fully stochastic systems for example Chow (1975b) and Kendrick and Majors (1974) to name just two of an ever expanding literature. The inclusion of parameter uncertainty in the fixed target framework presents some problems. The nature of the solution procedure makes learning techniques questionable in effectiveness. One technique would be to replace the coefficients by expected values and run monte carlo simulations to gain a knowledge of the variance in policy and target performance. The problem here would be that existence of policy could not be obtained in many cases whereas existence could be obtained in a purely deterministic framework. Optimisation techniques are clearly ahead of the fixed target approach in terms of parameter uncertainty but as this study constitutes the first attempt to employ fixed targets techniques, it will not be concerned with parameter uncertainty. Nonetheless it does suggest a very interesting area for future research. Of more interest is the reaction of the alternative solutions to additive uncertainty. The feedback nature of optimisation allows the controls to adjust past shocks and in this sense the technique is more flexible than the fixed target technique which in general cannot adjust to additive uncertainty as the past state of the system only enters into the solution once in the form of initial conditions prevailing at the beginning of the planning period. However, if strongly-Tinbergen conditions exist then the fixed target 
approach is able to adjust to past shocks as policies are computed for each time period without anticipation and it is only under these conditions that such adjustment can take place. The removal of past state behaviour from the computation of controls for target path achievement removes the problem of obtaining accurate information about the past State vector - a difficult problem for any real world application of optimisation techniques and one which has not been adequately explored in the literature. Both techniques suffer from the perhaps unpalatible assumption of perfect foresight about the future behaviour of the exogenous variables although filtering and adaptive techniques can be employed in optimisation solutions. The advantage of optimisation is that because it reflects a situation of zero anticipation and considerable compromise, it would, in the event of new information becoming available about future exogenous variables, be feasible to abandon the current plan and re-compute the optimal controls for the remaining of the planning period." This procedure would not be feasible in a fixed target framework unless the policy-maker was dealing with a strongly-Tinbergen situation as the need to re-plan would constitute hitting the targets outside of the current planning period due to the need to adequately anticipate the exact achievements of the targets. The answer to the question of which technique to use cannot be answered from theory or armchair speculation. Each stabilisation problem has its own characteristics which perhaps may favour one or the other technique. The applied results which are presented in the remainder of this study will hopefully shed some valuable light on this issue. 


\section{A SMALL OPEN MODEL OF THE AUSTRALIAN ECONOMY}

In this chapter a small open model of the Australian economy will be developed and estimated. The philosophy behind the construction of the model is that only the "minimal" size model required to illustrate the important linkages between the open, monetary and income sectors will be discussed. As is the practice with most econometric studies, a wide variety of differing structures were tested but because of the limited space available the alternative specifications will not in general be discussed. As we have indicated in the previous chapter, non-linear techniques are available for the approximate optimal control of non-linear models. However, as the dynamic Tinbergen approach requires a strictly linear model, we shall proceed directly to the construction of a linear model and disregard non-linear versions which may be linearised. The need to employ a linear model does place some restriction on the structure of the model and can cause difficulties through the mixture of real and nominal values of variables in equations. If the inherent non-linearities are not too severe however, then it could be expected that the choice of a linear version will not result in excessive information loss. The term "minimal" model needs some clarification at this point. The aim of the applied investigation is to observe the behaviour of the major broad aggregates rather than a multitude of subsectors. This can be effectively carried out by aggregating the system into a smaller system which captures all desired structural characteristics. Secondly, the use of very large models does not guarantee any additional information so the model presented here was originally formulated as a much larger version of the final structure presented and gradually reduced. Equations which did not contribute any significant 
influence on the general results were either simplified or deleted with the requirement that the remaining structure gave similar results to any preceding larger versions. On a more practical level, a large model would be very difficult to handle in the dynamic Tinbergen fixed target framework and as this study is the first to contain any applied analysis of dynamic fixed target problems, it is of some benefit to keep the analysis as simple as possible. Even with a small simple model, the solution of the fixed target problem presents a formidable computational problem as will be seen in chapter seven. The requirements of linearity, simplicity and smallness leave stabilisation studies of the kind presented here open to charges of "bending the problem to fit the technique" but the use of a small simple model does not necessarily imply any significant information loss at an aggregated level and as we have already pointed out a linearity requirement need not be too restrictive in all cases.

Before proceeding to a discussion of the model, a number of issues need to be considered. Firstly, the proliferation of optimal stabilisation studies has given rise to a debate concerning the endogeneity of government controls in estimated models. Clearly, if governments do have some kind of cost function in mind and controls are continually being adjusted to offset current stocks in order to minimise accumulated welfare costs, then controls are endogenous and should be treated as such in the estimation of models to avoid serious specification errors. Recent work by Crotty. (1973) (1976), Boddy and Crotty (1975) and Blinder and Goldfeld (1972) has focussed on a theoretical assessment of this problem. The possibility of such a feature raises some interesting econometric problems in regard to the degree of coefficient bias that may be present if the controls are not treated as endogenous. However, the adjustment of current controls to current deviations in the desired targets implies that the 
decision period for policy is shorter than the observation period for the data and that policy authorities are able to react to current shocks and observations. Clearly the authorities would not know the size and direction of shocks until after the data for the current period becomes available. In this case, it could be reasonably argued, depending on the time unit chosen, that policy must be formulated on the basis of past performance and as such controls become a function of predetermined variables and can legitimately be treated as exogenous. The use of feedback control laws also reinforces this as controls are always adjusted in response to past target deviations. The choice of unit time period is important in this case as there could be an argument for treating the controls as endogenous if annual data were used when the correct model is quarterly, as controls would most likely have been adjusted within the current time period. To avoid this specification problem, the time interval chosen was quaxterly although as we shall see below this can also lead to problems in a stabilisation framework. The fixed target approach presents some conceptual problems with respect to endogenous instruments as the current controls are a function of current desired target levels. It is not clear whether or not controls should be treated as endogenous for estimation purposes in this situation. However, as fixed target techniques have not yet been employed for stabilisation purposes, especially by present or past Australian governments, no specification exrors are likely to occur in the estimated model from this source. With the level of knowledge of stabilisation techniques now making control of national economies more feasible, it is of some importance to consider the possibility of specification error when choosing a stabilisation model. 
The linear/quadratic controls laws set out in Chapter Two utilised the reduced from of an economic system. Similarly, the fixed target solution can be derived directly from the reduced form. If the stabilisation techniques are amenable to the direct use of reduced forms then why bother carrying out a structural form estimation when the reduced form can be estimated directly? Monetarists argue that it is exceptionally difficult to model the transmission of policy, particularly monetary policy, and even a complicated model of considerable size will not be able to capture the underlying structure. As such, reduced forms should be estimated. Blinder and Goldfeld (1972) have shown that the direct estimation of reduced forms can seriously bias the estimated impacts of monetary and fiscal policy. Extending this to a stabilisation framework it is clear that given an incorrect assessment of the effectiveness of policy, an inappropriate mix of policy can result. Even when a structural form is specifically outlined, the direct estimation of a reduced form can be inconsistent with the underlying structure. The work of Blinder and Goldfeld places serious doubt on the acceptability of using reduced form models and in particular questions the findings of perhaps the most widely known monetarist model - the St Louis model (Andersen and Jordan (1970)). To avoid problems of reduced form estimation, the model will be estimated in its structural form and then the "true" reduced form will be obtained from the structure. While criticism of the structure can be made, the reduced form will be at least consistent with the structure and provided the structural form can adequately capture the general relative importance of monetary and fiscal policy, the reduced form instrument multipliers will display a similar tendency.

The model itself is essentially Keynesian in nature and assumes a fixed exchange rate, and is divided into three sections which feed back 
into and interrelate with each other. The monetary sector is closely related to the open sector through the concept of a money base and endogenous money supply. The inclusion of an endogenous money supply raises the interesting proposition that the planning authorities are unable to directly control the supply of money and hence it cannot be directly treated as an instrument as is the case in many econometric and theoretical studies. The monetary variable that the authorities can control directly is taken to be the domestic component of the total monetary base. The open sector is based on the portfolio approach to capital inflow developed by Porter (1974) for the Australian economy. Porter's work is used as the basis for building up the relationship between capital flows, foreign reserves, the money base and hence the money supply. The link between the income, monetary and open sectors is provided firstly through the effect of the rate of interest on investment and secondly, through a financial wealth effect on consumption. In keeping with the philosophy of simplicity, the controls have been restricted to two, a basic fiscal instrument (government spending) and a basic monetary instrument (domestic component of the monetary base). As it turned out, after considerable. experimentation with larger sets of instruments, the choice of the two finally included was something of a necessary condition for feasible policy solutions to exist, given the model. The exclusion of other "minor" instruments stems from the fact that in both the optimising (no adjustment costs on controls) and fixed target frameworks, the achievement or attempted achievement of specific target sets required that all instruments be used in a rigorous manner. For example, the statutory. Reserve Deposit ratio (SRD) was reguired to be used vigorously each time period (quarterly) with shifts of up to $100 \%$ per quarter and occasionally negative values being required. This behaviour was not confined to the 
S.R.D. ratio but was evident in other controls which typically are not used continuously by the authorities. The net result was that in the linear/quadratic solutions controls such as the S.R.D. ratio were weighted heavily to avoid adjustment. As these controls were not able to contribute substantially to the results without unrealistic policies resulting, they were abstracted from in the final form of the model. Such instrument behaviour has been found to occur in other stabilisation studies, for example, in the work of Pindyck (1973a), where it was found that the time path of the tax surcharge behaved in an unacceptable manner when it was allowed to adjust freely to complement the other controls in the system. Clearly, large quarterly shifts in tax rates or surcharges would not be tolerated in a real world situation. The behaviour of particular controls in either a flexible or fixed target framework indicates that the use of such techniques are reasonably restrictive and that what is required is a combination of formal techniques and short run discretion whereby instruments such as the S.R.D. ratio and tax rates can be adjusted in an appropriate manner. The control theorist would need to bear these considerations in mind when constructing a suitable "control" model. The abstraction from the S.R.D. ratio does not necessarily imply a weakness in the model as over recent years the Reserve Bank has shifted away from the use of direct controls such as the S.R.D. ratio with the main emphasis being on market oriented controls such as open market operations. This is not to say that the S.R.D. ratio is no longer used but its role has been substantially limited. In many cases it has been implemented solely for the purpose of mopping up seasonal variations in the supply of money. The tendency of trading banks to effectively circumvent direct controls, largely through market manipulations, has been the key force behind the move towards market 
controls. The incorporation in the model of the domestic component of the monetary base as the monetary control of concern effectively captures this feature.

The foundation of the income sector is the national income identity. This is given by

$$
Y=C+I+X-I M+G
$$

where $Y$ equals income, $C$ equals consumption, I equals investment, $G$ equals government spending, $\mathrm{x}$ equals exports and IM equals imports. $G$ is the fiscal instrument and exports are taken to be exogenous and uncontrollable. That is, it is assumed that the volume of exports largely depends on external factors and internal seasonal variations - a reasonable assumption in the case of Australia. The treatment of $\mathrm{x}$ as exogenous also recognises the fact that model builders have experienced considerable difficulty in constructing acceptable export functions. The remaining components of the income identity, C, I and IM are explained by behavioural relationships.

Previous macro models of Australia have tended to disaggregate consumption spending into two or more components. For example Higgins and Fitzgerald (1973), Kmenta (1966), Nevile (1970), Norton and Henderson (1972) and Zerby (1969). While this approach undoubtedly has its merits, it was found that doing so in this case did not substantially affect the overall general results and as such, a single aggregate function has been included. Modern macro theory has firmly established the importance of wealth in the role of consumption. A simple model which captures the earlier Keynesian concept of consumption and which incorporates wealth is given by

$$
C_{t}=a_{0}+a_{1} Y D_{t}+a_{2} W_{t}
$$


where YD equals disposable income and $\mathrm{W}$ represents private scctor wealth. A more sophisticated version of (3.2) is that presented by Ando and Modigliani (1963) which incorporates the effects of expected future income. The aggregated version of the Ando-Modigliani consumption function is given by

$$
C_{t}=a_{0}^{\prime}+a_{1}^{\prime} Y D_{t}+a_{2}^{\prime} w_{t}+a_{3} Y_{t}^{e}
$$

where $y_{t}^{e}$ equals expected future income. Attempts were made to estimate (3.3) but the presence of $y_{t}^{e}$ presented some difficulties. Various proxies were tested such as the use of a time trend of actual income (used by RBAl, Norton and Henderson (1972)) which were not completely satisfactory. $y_{t}^{e}$ can be removed from (3.3) by introducing a simplifying assumption that expected future income is a constant multiple of current household disposable income. Thus

$$
y_{t}^{e}=\beta Y_{t^{\prime}} \quad \therefore \beta>0 \quad .
$$

(3.4) asserts that if disposable income increases then people will adjust their expectations of the level of expected future income by a factor $\beta$. By incorporating (3.4) into (3.3) we have

$$
C_{t}=a_{0}^{\prime}+a_{1}^{\prime} \dot{Y D_{t}}+a_{2}^{\prime} w_{t}+a_{3}\left(\beta Y D_{t}\right)
$$

which can be written as

$$
C_{t}=a_{0}^{\prime}+a_{1}^{\prime \prime} Y D_{t}+a_{2}^{\prime} w_{t}
$$

where

$$
a_{1}^{\prime \prime}=\left(a_{1}^{\prime}+a_{3} \beta\right), \quad 0<a_{1}^{\prime \prime}<1
$$

which of course is in precisely the same form as (3.2) but allows for the general separation of future income effects from disposable income and provides a more acceptable basis for the estimation of a (3.2) type function. As it stands (3.5) can be estimated directly provided that 
appropriate wealth data can be found. Unfortunately, in the past good wealth data for Australia has not been available which makes estimation slightly more difficult. Theoretical work usually breaks wealth up into financial and physical wealth with physical wealth being represented by the capital stock. Without venturing into the well-known debate on whether or not physical capital can in fact be measured, it was decided to incorporate only financial wealth in the consumption function - a simplification successfully employed in RBAl. The exact definition of financial wealth provided some difficulty and a discussion of the definition is left until later. The inclusion of financial wealth allows for a direct monetary policy linkage with the income sector as a definition of financial wealth will necessarily include the supply of money in either of its various forms.

Like consumption, investment will be treated as a single aggregated variable. It has long been recognised that the estimation of an appropriate investment function is one of the most difficult aspects of macro model building as, unlike consumption theory in which a broad area of agreement has been reached, no settled form of the investment function has been settled upon. For example, should a neoclassical type function be incorporated or should an accelerator type: function be used? Neo-classical functions have found considerable favour, with a broad spectrum of economists but are notoriously difficult to estimate with considerable concessions to the theory being necessary in many cases to obtain an acceptable estimated function. The empirical work on investment has been limited in Australia but recent work by Higgins et al (1976) has thrown some doubt on the total effectiveness of neo-classical functions. Higgins et al concluded that the much maligned accelerator version of the investment function provides an 
explanation and prediction of investment as good as, and in some cases superior to, alternative specifications in relation investment behaviour in Australia over recent years. As such, and due to the difficulties of quantifying some of the neo-classical conepts, an accelerator type function was estimated. The structural form of the function was derived by initially distinguishing between investment expenditure and investment expenditure decisions. Investment expenditure refers to the actual expenditure that occurs at time $t$ while expenditure decisions refers to decisions to undertake specific projects, those decisions to be made from time $t$. If a firm wishes to expand its fixed capital it is reasonable to assume that it is unable to carry out all projects relevant to that expansion in the unit time period. Specifically, it is assumed that actual investment expenditure is carried out over a number of time periods with some fraction carried out in the first time period, some fraction in the second and so on. Putting this another way we can say that current investment is related to investment decisions made prior to the current time period. Using this concept and expressing investment as a distributed lag function and using the familiar accelerator assumption relating the desired capital stock to income in the following way

$$
K_{t}^{*}=\alpha Y_{t}^{\prime}
$$

we can derive a generalised accelerator function of the form

$$
I_{t}=k_{0}+k_{1}\left(Y_{t-n}-Y_{t-n-1}\right)+\sum_{t=1}^{j} c_{j} I_{t-j}
$$

where $\mathrm{n}$ reflects the lag on the actual income accelerator and $j$ represents the lag on the stock adjustment variables. Specific lags can be established a priori from specific assumptions about the lag structure of the decision process and the way in which terms are grouped together. However, in general the actual length of the lags involved 
will be an empirical question and it is possible that some components in the stock adjustment summation will not be statistically significant. In general, (3.9) represents an accelerator stock adjustment model in which it has been assumed that it is not possible to fully adjust capital stock to changes in output in one period. In its present form (3.9) provides a linear relationship which can be easily estimated but omits the important financial link with the monetary sector. The accelerator coefficient can be regarded as also reflecting the cost of finance, the tax structure and other restrictions on the ability of firms to undertake investment. Accordingly, financial effects in the form of the rate of interest can be separated and explicitly included in the function, presumably with a long lag. Thus the term

$$
b r_{t-s}, \quad b<0
$$

can be incorporated into (3.9) with s being the length of the interest rate lag on investment which once again is an empirical proposition. The company tax structure has not been explicitly included for the reasons outlined above - specifically because the solution techniques require adjustment every time period when it is only usual to adjust tax rates once every four quarters at the most.

Exports, as we have argued, can be legitimately treated as exogenous in Australia. In contrast, imports are a leakage from the system which depends very much on internal factors but like exports, adequate import functions axe difficult to estimate. While there is no general formal body of theory in the same vein as consumption or investment, it is possible to build an acceptable import function although in many cases variables are introduced in an ad hoc manner and tested in an empirical framework. As imports represent that portion 
of income not spent on domestic goods or saved, it is reasonable to assume that imports will depend largely on the level of domestic income which provides a basis for building up the remainder of the function. Imports are often viewed as a "safety valve" in that any excess demand which cannot be met by domestic production will spill over on to imports, thus relieving pressure on the system. It seems desirable then to include some measure of domestic capacity into our import function. Initially, experiments were carried out using a variable that measured capacity utilisation. Capacity utilisation is defined as the difference between registered vacancies and the total of unemployed persons divided by registered vacancies. The inclusion of such a variable failed to contribute significantly to the overall performance of the model in either its non-linear or linearised versions. The result can be partially explained by the fact that excess demand was not significant over the estimation period - a conclusion which is supported by the stability of prices over the same period. A further influence on the level of imports is the degree of government control over imports. It is very difficult to capture the complexity of government import controls in a simple function and as such it is convenient to introduce a proxy variable for the governments external policy actions (excluding monetary policy). This approach is supported by the fact that historically, import controls in various forms have not been a major instrument of short run stabilisation in Australia and their explicit inclusion in the model would result in the type of unsatisfactory behaviour outlined above. Kmenta (1966) has suggested using lagged exports as an indicator of government's policy actions. The suggestion is based upon considerable experimentation with the data and reflects the notion of the role of exports as an indicator of the economic health of system and the assumption 
that government policy relating to imports is based on the behaviour of export income. Implicit in this approach is the notion that the government has some balance of trade (and possibly a balance of payments) objective and attempts some modest adjustment in imports in response to the behaviour of exports. An additional hypothesis can also be associated with the use of exports and that is that the past level of exports provides income and foreign exchange to the export sector which is then available for the purchase of imports. ${ }^{11}$ To complete the import function, relative prices are introduced although in a linearised version and a stock adjustment hypothesis is included which asserts that current imports are a function of past demand. The final version of the import function is given by

$$
I M_{t}=f\left(Y_{t}, X_{t-P}, I M_{t-1}, R_{t-m}\right)
$$

where IM equals imports, $\mathrm{X}$ equals exports and $\mathrm{RP}$ represents relative prices which were linearised in the estimated version. $\mathrm{P}$ represents the lag associated with the effect of exports and $M$ is the lag associated with relative prices.

As well as forming an important integral part of the income identity, exports and imports constitute a significant portion of a major external relationship, the current account balance. The identity for the current account balance $(\mathrm{CB})$ is given by

$$
\mathrm{CB}=\mathrm{X}-\mathrm{IM}+\mathrm{NT}+\mathrm{GF}
$$

where NT equals net transfers overseas and GF equals government capital flows. GF is included to keep (3.12) in line with the definitional requirements of Poxter's capital flow equation discussed below. The inclusion of GF in $\mathrm{CB}$ does not affect the overall balance of payments results as its inclusion simply means that the balance of payments 
position can be obtained by adding $\mathrm{CB}$ to private capital inflow.

To complete the income sector of the model we require a

relationship to explain personal disposable income, YD. By seeking to explain YD we also introduce supporting relationships for total personal income, TPY, and personal income tax payments, T. YD is defined by the following identity,

$$
\mathrm{YD}=\mathrm{TPY}-\mathrm{T}-\mathrm{OT}-\mathrm{IP} \text {. }
$$

OT equals other direct taxes (as distinct from $T$ ), fees, fines and other payments to government while IP equals interest payments on consumer debt. OT and IP are treated as exogenous variables. The treatment of the largest component of the identity, TPY, requires some consideration. As defined in the Quarterly Estimates of National Income and Expenditure published by the Australian Bureau of Statistics, TPY is equal to the sum of wages, salaries and supplements, income of farm unincorporated enterprises, income of other unincorporated enterprises and from dwellings and interest dividends, transfers from overseas and cash benefits from general

government. TPY would usually be incorporated into a model defined as above and treated as an identity with a wage/price sector explaining the evolution of wages, salaries and supplements. While the theoretical treatment of wage/price sectors has reached a level of considerable sophistication, for example, Turnovsky and Pitchford (forthcoming), the empirical formulation of such functions has not progressed to the same level, especially in Australia where researchers have in the past found it extremely difficult to adequately model wage/price sectors due to the nature of minimum wage fixation, the high level of unionism and the arbitration system. For example, Higgins and Fitzgerald (1973) treated money award wages covered by Arbitration Commission decisions as 
exogenous. This would include the majority of wages and salaries included in the definition of TPY. Due to the difficulties of constructing an adequate wage/price sector for Australia (since the introduction of wage indexation the modelling of the wage structure would become considerably easier) and as wages and prices were extremely stable of the estimation period, it was decided to exclude a sophisticated wage/ price sector in the model in favour of a more simplified treatment. Indeed, any attempt to estimate a detailed wage/price sector would constitute a major study in itself. A simple function relating TPY to national. income has been included in the model which is sufficient to capture the essential movement in total personal income in a period of wage and price stability.

To complete the identity for personal disposable income and the income sector as a whole, we require a relationship for personal income tax payments, T. The main influences on the level of personal income taxes are obviously the level of total personal income which is subject to taxation and of course the statutory tax rates which apply to such income. Other important closely related factors include the distribution of total personal income which is taxable and in the case of Australia, the degree of progressiveness of the tax structure. The notion of a progressive tax structure suggests that a non-linear relationship would be the most appropriate but this possibility is of course eliminated due to the linearity requirements of the model and it is of course very difficult to capture all the above factors without incorporating a large disaggregated tax subsector in the model similar to that constructed by Mackrell (1970) for RBAl. In fact, the use of a linear structure prevents the explicit inclusion of tax rates in the model in a form in which they can be treated as controls. This highlights 
a significant drawback of the linear approach but is counter-balanced by the earlier observation that as tax rates are not usually constantly manipulated, the inclusion of them in a fixed or flexible target stabilisation framework could lead to infeasible policies being specified. Empirical evidence of this type of behaviour can be found in wells (1977). The only alternative to treating total tax payments as an instrument which is clearly incorrect, would be to incorporate a tax surcharge instrument in a similar way to that employed by Pindyck (1973). This can be achieved by fitting a simple linear relationship between tax payments and total personal income but forcing the function through the origin and hence excluding an estimated constant term. The surcharge can then be added to the function as a constant term which can be directly manipulated by the government. Considerable experimentation was carried out with this type of function but was excluded from the final structural form due to the fact that most solutions required unrealistic shifts in the surcharge and to avoid this problem the surcharge had to be weighted heavily in the linear/quadratic framework and fixed a priori in the fixed target. framework and treated as an exogenous variable. The final form of the tax function was a simple linear function relating tax payments to total personal income

$$
T_{t}=t_{0}+t_{I} T_{t}
$$

with $t_{1}$ representing an average tax rate for the entire economy which of course remains fixed. This is not an unreasonable approach in light of past experience in Australia where governments have displayed considerable reluctance to change tax rates and use tax rates as an instrument of short run stabilisation. (3.14) adequately approximates the progressive tax structure, over the estimation period as the "explosion" in incomes 
relevant to recent Australian history did not occur until after the estimation period (pre 1972). It should be recognised that in a time of rapidly increasing incomes, (3.14) would most likely be considerably less than adequate.

In the past it has been customary to treat the supply of money as a control variable subject to direct manipulation by the monetary authorities. If this assumption is dropped we are left with a more realistic and interesting proposition that the supply of money is a variable which is endogenously determined within the system. The implications of this for economic policy are very important. Specifically, if the authorities require a particular target rate of growth of the money supply then it cannot be guaranteed that this target will be automatically achieved. The money supply function incorporated in the model presented here is based on the work of Teigen (1964), although some slight modifications to Teigen's work have been included. The significant departure from Teigen concerns the introduction of a foreign component of the money base which allows for the important interaction between the money supply and the open sector. The total monetary base is given by

$$
M B=D M+F R
$$

where DM constitutes the domestic component of the base and FR is the foreign component with FR defined as the level of foreign reserves. The government can inject or withdraw money into or out of the banking system by manipulating DM through open market operations. By building up the relationship between the required reserve ratio and deposits, the excess reserves of the banking system and the foreign component of the base a money supply function of the following form can be obtained. 


$$
M^{S}=\psi\left(D M+F R-R_{e}\right)
$$

with

$$
\psi=\frac{1}{s \cdot(1-d)+d}
$$

and where $R_{e}$ equals excess reserves, $S$ equals the required reserve ratio and d equals the proportion of the money supply held as notes and coins. In Australia, the required reserve ratio can be considered to be the Statutory Deposit Ratio. The supply of money is now expressed as a simple multiple of the money base less excess or free reserves with $\psi>1$ reflecting the multiple expansion effect of the banking system. A further extension to (3.16) can be introduced through the profit maximising behaviour of banks which is reflected in the supply of money having a positive interest elasticity. The positive response of the money supply to the rate of interest reflects the willingness of banks to try and issue more loans as the interest rate increases. In terms of (3.16) this is equivalent to expressing excess reserves as a function of the rate of interest giving

$$
M^{S}=\psi(D M+F R-f(r)) .
$$

Two estimation choices are open. Firstly, $\psi$ can be considered in a broken down version (3.17) in which it is desired to explicitly include $s$ in the estimation and would allow for $S$ to be treated as a control. The requirement of linearity negates this first approach. To overcome the problem of incorporating $S$, a linear approximation can be used whereby $\mathrm{S}$ appears as an explanatory variable in a linear fashion. Secondly, (3.18) can be estimated directly leaving $S$ as function of $\psi$. $\psi$ can be found by hand by fitting a straight line through the origin although after considerable experimentation it was found that a least squares 
estimation was more efficient. A further extension to the basic function was employed in which the rate of interest was removed from the brackets in (3.18) and treated as a separate explanatory variable - this procedure of course requires a least squares estimate. The final form selected for inclusion in the model consisted of the following specification,

$$
M^{S}=m_{0}+m_{1} M B+m_{2} r m_{1}, m_{2}>1
$$

The S.R.D. ratio was excluded on similar grounds to some forms of tax rates, that is, the ratio is not generally subject to large shifts for short run purposes and the stabilisation techniques require this to occur unless it is fixed. When it is fixed it fails to contribute to the overail controllability of the system and can be effectively excluded. The explicit exclusion of $S$ does not significantly affect the overall explanatory behaviour of (3.19) and of course $S$ is implicitly included in the function as the money multiplier, $m_{1}$, is a function of $s$.

The specification of a money demand function is much more straightforward with money demand, $\mathrm{M}^{\mathrm{d}}$, being a function of income and the rate of interest and following Goldfeld (1973) and other researchers, $\mathrm{M}^{\mathrm{d}}$ is specified to be a function of wealth. Thus

$$
\begin{aligned}
& M^{d}=L(Y, r, W) \\
& L_{1}>0, L_{2}<0,0 \leq L_{3} \leq 1 .
\end{aligned}
$$

Various lag structures and specifications were tested within the basic framework of (3.20). The specification of a suitable wealth variable presented some difficulties, remembering of course that a similar problem was encountered with the consumption function. The solution of the problem is discussed below. The sub-system of the model consisting of the demand for and supply of money can be closed by introducing the identity 


$$
M^{S}=M^{d}=M \text {. }
$$

The relationship between the rate of interest and the supply of and demand for money is crucial with the rate of interest being determined by, and at the same time determining, $\mathrm{M}^{\mathrm{S}}$ and $\mathrm{M}^{\mathrm{d}}$ in a simultaneous manner. If we invoke (3.21) and insert $M$ into (3.20), the function for the rate of interest can be derived directly from the money demand function. Hence

$$
\begin{aligned}
& x=L^{*}(Y, M, W) \\
& L_{1}^{*}>0, \quad L_{2}^{*}:<0, \quad 0 \leq L_{3}^{*} \leq 1 .
\end{aligned}
$$

Up to this point in the discussion "the" rate of interest has been referred to. The question is, which rate of interest should be incorporated in the equations (3.19), (3.20) and (3.22) and should it be the same as the rate of interest included in the investment function? Clearly, the rate of interest included in the investment function should be a long rate. At first sight, the choice of interest rate for the money supply and demand functions and hence (3.22), is not as straightforward. The best we can do is appeal to the available body of empirical research which exists on this subject as a detailed exhaustive examination of the issues and problems involved is beyond the scope of this study. Following other researchers such as Heller (1965), Laidler (1966), Zerby (1969) and Adams and Porter (1976), a short rate will be included in the money supply and demand functions as short rates tend to reflect the opportunity cost of holding money instead of close substitutes. The choice of a short rate as the appropriate rate to include presents some further difficulties as there are a multiplicity of rates to choose from and any rate which is chosen will only serve as an indicator or proxy for movements in the short term 
market. The rate chosen was the yield on two-year government bonds as this rate is influenced by changes in the Liquid Assets and Government Securities (L.G.S.) assets of the commercial banking system and is also influenced by shifts in the liquidity preference of the non-bank public. The inclusion of a long rate and a short rate requires a behavioural equation explaining the long rate. This is most effectively carried out by specifying a simple term structure relating the long rate to the short rate. To some extent the long and short rates have in the past exhibited a tendency to move broadly in line with each other with the long rate generally lying above the short rate. This proposition can also be established theoretically, see Lutz (1968), for example. The ten-year government bond rate was used as a proxy variable for "the" long rate of interest.

Before discussing the remaining equations of the model it is convenient to return to the question of an appropriate definition of wealth and the related area of an appropriate definition of the supply of money. Most definitions of wealth include the supply of money and the holding of other financial assets such as government bonds. Considerable experimentation was carried out using various definitions of wealth with the limited amount of data that was available at the time of estimation. It was found that a broad definition of money was adequate as a definition of financial wealth with the addition of other variables not adding significantly to the overall estimation or the results of various control experiments. As such, only money was included. The money demand and interest rate equations were adjusted accordingly. This procedure also had the advantage of decreasing the size of the system as the inclusion of other components of wealth required corresponding behavioural relationships. There appeared to be little gained from specifying 
additional relationships for variables which did not contribute significantly to the analysis. Unlike other variables such as $C$ and I where the choice of data is relatively straightforward, there is no settled body of opinion on what constitutes the "correct" definition of money. As financial wealth has to some extent been abstracted from, the definition of money employed here is broader than is usually considered. In addition to currency and demand deposits with trading banks, fixed term deposits (including certificates of deposit with all trading banks) plus deposits with all savings banks have been included. Thus the definition includes assets with perfect liquidity and assets with a less degree of liquidity, depending on the withdrawal restrictions imposed by the trading banks and if only currency and term deposits are taken to be the usual constituents of "money" then the remaining assets can be regarded as "near money". As we have already indicated however, the broad definition proved to be the most acceptable in terms of the overall philosophy of the model.

The basic idea behind the capital flow equation (Porter (1974)) is the notion that capital flows provide the means by which the private sector is able to remove any deficiency in the amount of. liquidity (in this case money) it requires. Thus, a positive change in national income will generate an increase in the demand for liquidity which can be partially satisfied through capital flows. Similarly, a negative current account balance will induce a currency drain in the private sector. The resulting outflow of foreign reserves will cause a fall in the stock of money through its influence on the total monetary base. The net result of this movement will be a balancing effect from an inflow of capital. The reverse situation is also true. If the current account balance is positive then the resulting flow of foreign reserves into the 
system will result in capital inflow not being required to help satisfy liquidity in the private sector. The capital inflow function also incorporates the change in the Euro-dollar rate which serves as a proxy for foreign alternatives for the allocation of funds by overseas investors. We would therefore expect a positive change in overseas rates to have a negative impact on capital inflow in Australia as funds are diverted elsewhere. Porter's equation also includes a variable representing speculative inflow - a variable which has been of considerable importance in recent Australian history. The inclusion of a speculation variable implies "that any expected change in the exchange rate is a function of the level of foreign exchange reserves at the beginning of the period; once reserves reach a certain initial "trigger" value."(Porter (1974,p.7)). By specifying a particular "trigger" value we could expect some simultaneous feedback from the level of foreign reserves to capital inflow which would then feedback into foreign reserves and so on. Porter's original investigation was within the confines of a single equation analysis. The exterision to a multi-equation system allows for the situation in which a blow-up in foreign reserves induced by speculation will result in a significant impact on the supply of money and income through the wealth effect in consumption. Finally, the effect of government generated liquidity is included in the function through the introduction of the domestic component of the monetary base, DM. An increase in base money will have a direct increase on $M B$ and $M$ by definition. However, a positive change in domestic base money will also have a negative impact on capital flows (CF) which, depending on the movement of the current account balance, could cause a downward shift in the level of foreign reserves. Thus we have a potentially selfdefeating monetary policy situation in which the authorities may try to 
expand the money supply by a domestic monetary expansion which could be offset to some degree by a corresponding drain on foreign reserves. It is this relationship between the sectors which provides some of the most important and novel interactions of the model and it is this aspect of the model which appears to be unique to Australian models which have not adequately modelled the interactions between the open and monetary sectors. of course, the degree to which monetary policy is compromised depends on the offset coefficient associated with DM in the capital flow equation. The general form of the capital flow equation is given by 12

$$
\begin{aligned}
& C F_{t}= f_{0}+f_{1} C B_{t}+f_{2} \Delta D M_{t}+f_{3} \Delta Y_{t}+f_{4} S C_{t}+f_{5} E R O \\
& \\
& f_{1}<0, f_{2}<0, f_{3}>0, f_{4}>0, f_{5}<0 .
\end{aligned}
$$

where $\mathrm{SC}_{t}$ equals the speculative proxy and $\mathrm{ERO}_{t}$ equals the change in the Euro-dollar rate. The open sector and the linkage with the monetary sector is completed by the addition of the identity

$$
\triangle \mathrm{FR}=\mathrm{CB}+\mathrm{CF}
$$

For simulation purposes and to provide for the feedback of FR into the total monetary base, $(3.24)$ can be written as

$$
F \dot{R}_{t}=\mathrm{CB}_{t}+\mathrm{CF}_{t}+F R_{t-1}
$$

A major difficulty associated with the model was the choice of deflated or nominal data to be used in the estimation of the structural equations. On the income side, real national income, real investment and real consumption are more important than the corresponding nominal values. However, if we estimate the model in real terms and then carry out policy experiments we are implicitly assuming that the authorities are able to exercise direct control over real government spending and real domestic base money. To some extent this is an invalid assumption 
as governments are only able to directly control nominal values of instruments. In the monetary and external sectors it is usually nominal values which are important, at least in Australia. It is generally the nominal values of foreign reserves, capital flows and money supply which are of interest. The treatment of some variables as real and using linear approximations of others to give nominal values (for example $\left.\alpha \frac{M}{p} \simeq \alpha_{1} M+\alpha_{2} p\right)$ gives an unsatisfactory mix of real and nominal variables in the model. The final version of the model was estimated in nominal terms and then required variables were deflated by a simple price equation. This procedure was effective due to the low rates of price inflation over the estimation period. Initially, a simple supply curve function was estimated

$$
\mathrm{P}_{t}=\alpha_{0}+\alpha_{1} \mathrm{y}_{t}
$$

where $y_{t}$ equals real national income. The inclusion of (3.26) presented the problem of incorporating real income when income was treated as nominal in the remainder of the system. Recognising that (3.26) can rewritten as

$$
\mathrm{P}_{t}=\alpha_{0}+\alpha_{1}\left(\frac{\mathrm{Y}}{\mathrm{P}_{t}}\right)
$$

a linear approximation can be taken where $\alpha_{1}\left(\frac{Y}{P}\right)$ can be expressed as $\alpha Y+\alpha P$. This gives a form of the function

$$
P_{t}=\alpha_{0}^{\prime}+\alpha_{1}^{\prime} Y_{t}
$$

(3.28) is admittedly a crude relationship but its inclusion does not affect the evolution of the remainder of the system. Australia has had a long tradition of imported inflation (see Pitchford (1968)) and it: was desirable to include some imported component of the price level to enable some sensitivity analysis to be carried out, if required. Thus the function for the price level becomes 


$$
P_{t}=\alpha_{0}^{\prime}+\alpha_{1}^{\prime} Y_{t}+P_{t}
$$

where $\mathrm{OP}_{t}$ represents a proxy variable for the imported component of inflation. It is stressed that (3.29) is only meant to provide a deflating or scaling procedure for the remainder of the system and does not affect system performance or the trade-offs between the variables. Indeed, if all variables are treated as real variables then the resulting estimated structure would give the same general policy results and implications as the nominal version. However, if we were still interested in nominal values of particular variables then some procedure for inflating the data would be required and a function of the form of (3.29) would be appropriate in that case as well. The recent burst in inflation would probably invalidate (3.29) and a comprehensive wage/price sector would need to be incorporated into the model. ${ }^{13}$ However, the stability of wages and prices over the selected estimation period results in (3.29) being an adequate proxy for a price generator.

It was indicated above that the unit time period selected for the model was quarterly which is the usual time period chosen for short run stabilisation and also it reduces the change of the specification error outlined above. There is one difficulty, however, associated with the use of a quarterly model when open and monetary sectors are incorporated along with an income sector. The problem is that while income data is typically only available in annual or quaxterly form, monetary variable information is available on a monthly and sometimes weekly basis. Thus, in reality, the monetary authorities could be adjusting their instruments on a monthly basis giving very close short run stabilisation with respect to those variables. The fiscal authorities on the other hand may be forced to operate stabilisation policy within at least a quarterly unit time period. ${ }^{14}$ Clearly, an annual model would be totally unacceptable 
for stabilisation purposes when an open and monetary sector is included. The best that can be done is to use a quarterly model while at the same time recognising that there could be some information and controllability loss in the open and monetary sectors in practice. Ideally, if the true monetary model is say monthly and the appropriate fiscal model is quarterly, we would like to have an interaction between sectors in which each sector is modelled within the appropriate unit time period. This would be very difficult except under very stringent and restrictive conditions. Further analysis related to this issue can be found in later chapters.

\section{MODEL ESTIMATION}

The complete estimated version of the model is listed below. It should be noted that a great number of structures were tested within the basic framework outlined above. The final version of the model is that which satisfies the basic philosophy and the minimality condition in the most appropriate fashion. Even within a basic Keynesian framework there are a number of alternative specifications which could be regarded as acceptable. Stabilisation experiments were conducted with a variety of specifications with the outcome that the results presented in the following chapters appear to be quite robust in relation to alternative structures similar to the overall philosophy of the model. The data sources were the Australian Bureau of Statistics Quarterly Estimates of National Income and Expenditure and the Reserve Bank's Statistical Bulletin. The structural equations were estimated by two stage least squares using quarterly seasonally unadjusted nominal data. The estimation period ranged from the second quarter 1965 to the fourth quarter 1972. Scasonal dummies were included in each structural equation. The bracketed figures under the coefficients are $t$ statistics. D.W., S.E.R. and $R^{2}$ refer to the Durbin-Watson statistic, the standard error of the residual and the 
cocfficient of determination respectively.

National Income

$$
Y=C+I+X-I M+G
$$

Total Monetary Base

$$
\mathrm{MB}=\mathrm{DM}+\mathrm{FR}
$$

Change in Foreign Reserves

$$
\Delta \mathrm{FR}=\mathrm{CF}+\mathrm{CB}
$$

Current Account Balance + Government Capital Flows

$$
\mathrm{CB}=\mathrm{X}-\mathrm{IM}+\mathrm{GF}+\mathrm{NT}
$$

Personal Disposable Income

$$
Y D=T P Y-T-O T-I P
$$

Total Personal Income

$$
\begin{array}{ccc}
\operatorname{TPY}_{t}= & -317.073-395.21 \mathrm{~S} 1-141.69 \mathrm{~S} 2+126.37 \mathrm{~S} 3+.846 \mathrm{Y} \\
& (-3.324) \quad(-6.861) \quad(-2.470) & (2.142) \\
\mathrm{R}^{2}=.99 \quad \mathrm{DW}=2.262 & \mathrm{SER}=110.516
\end{array}
$$

Personal Consumption Expenditure

$$
\begin{gathered}
C_{t}=166.565+106.34 S 1+94.01352+.601 \mathrm{YD}_{t}+.053 \mathrm{M}_{t}+.1202 \mathrm{C}_{t-1}(3.36) \\
\begin{array}{ccccc}
(1.638) & (2.673) \quad(2.674) & (11.122) & (2.000) & (1.240) \\
R^{2}=.99 & D W=2.19 & S E R=76.169
\end{array}
\end{gathered}
$$

Private Investment

$$
\begin{aligned}
I_{t}= & 124.761+90.329 S 2+.1489\left(Y_{t}-Y_{t-1}\right)-24.903 R L_{t-4}+.52 I_{t-1} \text { (3.37) } \\
& (1.705) \quad(3.376) \quad(7.027) \\
& +.473 I_{t-2} \\
& (3.483) \\
& R^{2}=.98 \quad D W=1.942 \quad S E R=37.06
\end{aligned}
$$


Imports of Goods and Services

$$
\begin{aligned}
& \mathrm{IM}_{t}=1127.559+33.252 \mathrm{~S} 2-48.165 \mathrm{~S} 3+.0622 \mathrm{Y}_{\mathrm{t}}+.212 \mathrm{x}_{\mathrm{t}-3} \\
& \begin{array}{lllll}
(3.913) \quad(2.007) \quad(-2.311) \quad(3.396) & \text { (1.503) }
\end{array} \\
& -12.2170 \mathrm{P}_{\mathrm{t}}+.561 \mathrm{M}_{\mathrm{t}-1} \\
& (-3.435) \quad(4.978) \\
& \mathrm{R}^{2}=.95 \quad \mathrm{DW}=1.711 \quad \mathrm{SER}=37.38
\end{aligned}
$$

Supply of Money

$$
\begin{aligned}
& M_{t}=1168.311-569.317 \mathrm{~S} 1+2.743 \mathrm{MB}_{t}+722.51 \mathrm{RS}_{t} \\
& (1.087) \quad(-2.263):(21.132) \quad(3.640) \\
& \mathrm{R}^{2}=.94 \quad \mathrm{DW}=2.262 \quad \mathrm{SER}=610.883
\end{aligned}
$$

Short Rate of Interest

$$
\begin{array}{rl}
\mathrm{RS}_{t}= & 5.512-.675 \mathrm{~S} 1-.474 \mathrm{~S} 2-1.189 \mathrm{~S} 3+.00122 \mathrm{Y}_{\mathrm{t}} \\
& (10.139)(-3.131)(-3.089)(-5.384) \quad(5.271) \\
-.00059 \mathrm{M}_{t}+.848\left(\mathrm{RS}_{t-1}-\mathrm{RS}_{\mathrm{t}-4}\right) & \\
(-4.202) & (11.028) \\
\mathrm{R}^{2}=.85 & \mathrm{DW}=2.452 \quad \mathrm{SER}=.218
\end{array}
$$

Long Rate of Interest

$$
\begin{gathered}
\mathrm{RL}_{t}=.159+.091 \mathrm{SI}+.054 \mathrm{~S} 2+\underset{(1.239)}{(1.975)}\left(\begin{array}{c}
.394 \mathrm{RS}_{t}+.599 \mathrm{RL}_{\mathrm{t}-1} \\
(1.228)
\end{array}\right. \\
\mathrm{R}^{2}=.96 \quad \mathrm{DW}=2.344 \quad \mathrm{SER}=.1003
\end{gathered}
$$

Private Capital Flows

$$
\begin{aligned}
& \mathrm{CF}_{t}= 152.125-118.478 \mathrm{~S} 2-69.442 \mathrm{~S} 3-.597 \mathrm{CB}_{t}-.259\left(\mathrm{DM}_{t}-\mathrm{DM}_{t-1}\right) \\
&(4.018) \quad(-4.217) \quad(-2.514) \quad(-3.910) \quad(-2.943) \\
&+ .048\left(\mathrm{Y}_{t}-\mathrm{Y}_{\mathrm{t}-1}\right)+.089 \mathrm{SC} t-27.49 \mathrm{ERO}_{\mathrm{t}} \\
&(2.283) \quad(7.184) \quad(-1.973) \\
& \mathrm{R}^{2}=.86 \quad \mathrm{DW}=2.544 \quad \mathrm{SER}=32.626
\end{aligned}
$$


Personal Income Tax Payments

$$
\begin{array}{r}
\mathrm{T}_{\mathrm{t}}=-191.336-14.366 \mathrm{~S} 1-13.062 \mathrm{~S} 3+.154 \mathrm{TPY} \mathrm{t}_{\mathrm{t}} \\
\begin{array}{r}
(-12.441) \\
\mathrm{R}^{2}=.99 \quad
\end{array} \quad \mathrm{DW}=2.242 \quad \mathrm{SER}=19.653
\end{array}
$$

Price Equation

$$
\begin{gathered}
\mathrm{P}_{t}=51.278-4.331 \mathrm{~S} 1-.546 \mathrm{~S} 2-5.176 \mathrm{~S} 3+.0068 \mathrm{Y}_{t}+.13370 \mathrm{P}_{\mathrm{t}} \\
\begin{array}{c}
(6.718) \quad(-7.148) \quad(1.682) \\
(-7.808) \quad(16.596)
\end{array} \\
\mathrm{R}^{2}=.99 \quad \mathrm{DW}=2.136 \quad \mathrm{SER}=1.109
\end{gathered}
$$

\section{Endogenous Variables}

$C=$ Total personal consumption expenditure.

$\mathrm{CB}=$ Current account balance plus government capital flows.

$\mathrm{CF}=$ Net private capital inflow.

$\mathrm{FR}=$ Foreign reserves.

$I=$ Private gross fixed capital expenditure.

IM $=$ Imports of goods and services.

$M=$ Volume of money (currency plus current deposits with all trading banks plus fixed term deposits, including certificates of deposit with all trading banks, plus deposits with all savings banks).

$\mathrm{MB}=$ Total monetary base.

$\mathrm{P}=$ Price level (implicit GNP deflator).

$\mathrm{RL}=$ Long rate of interest (10-year government bond rate used as a proxy variable).

$\mathrm{RS}=$ Short rate of interest (2 year government bond rate used as a proxy variable).

$\mathrm{T}=$ Personal income tax payments.

TPY = Total personal income.

$\mathrm{Y}=$ National income.

$\mathrm{YD}=$ Personal disposable income. 


\section{Exogenous Variables}

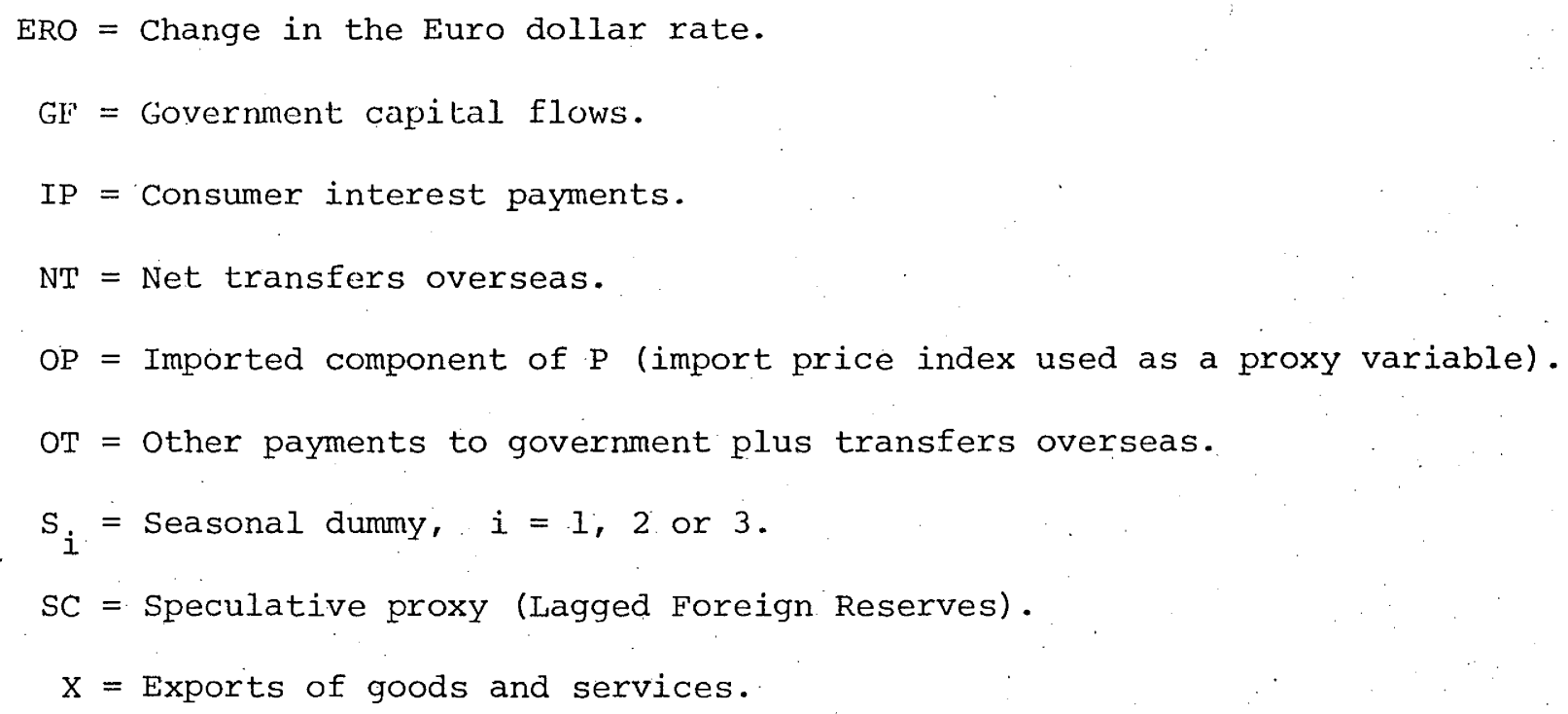

\section{Controls}

DM = Domestic component of the monetary base defined as domestic assets of the Reserve Bank.

$G=$ Government expenditure (consumption plus fixed capital expenditure).

Despite the overall size and simplicity of the model, the estimated results are quite satisfactory. The signs of all coefficients correspond to those specified by economic theory. Note that the money demand function has been deleted from the listing of the structural equations. This is because its presence did not significantly contribute to the overall performance of the model as the money demand and short rate of interest equations are essentially the same. To include both would be unnecessarily repetitive. As a check, both equations were estimated and compared. It was found that when the money demand function was rearranged to yield an interest rate function, the coefficients were very close to the estimated coefficients for the interest rate equation. One other aspect of interest was the failure of significance of domestic prices in the import function. Recall that a linear approximation of relative prices was incorporated in the import function. The failure of significance 
may not be solely due to the linearisation as previous studies, for example, Gregory and Martin (1976), have indicated that relative prices in a non-linear form have not performed particularly well in estimates of import functions.

To gain some insight into the dynamic performance of the model, a dynamic simulation was carried out using historical data for the initial conditions, exogenous and control variables. The results are summarised in figures two to five. Y has been chosen to summarise the behaviour of the income sector and FR indicates the dynamic performance of the open sector. The money supply and long rate of interest are also graphed. It is clear that the model performs exceptionally well in relation to the income sector and effectively captures the general behaviour of the open sector. The model is especially effective in capturing the sharp upturn in foreign reserves experienced towards the end of the estimation period. The performance of the open sector is particularly encouraging in light of the fact that the coefficient of determination for the capital flow equation is only .86 . While the $\mathrm{R}^{2}$ of an equation should not be the sole criterion for judging the acceptability of an equation it is desirable to obtain a good fit to the data as one "bad" equation which has a substantial effect on othex sectors can seriously affect the performance of the model with respect to replicating the system which it purports to present. The performance of the open sector is further enhanced by the simulated level of the current account balance which tracks very close to its historical time path. Similarly, the short rate of interest equation does appear to be potentially of some concern. As in the case of the capital flow equation, however, the simulated levels of RS reflect the general underlying behaviour of the historical data and therefore help to keep the long rate largely on track 


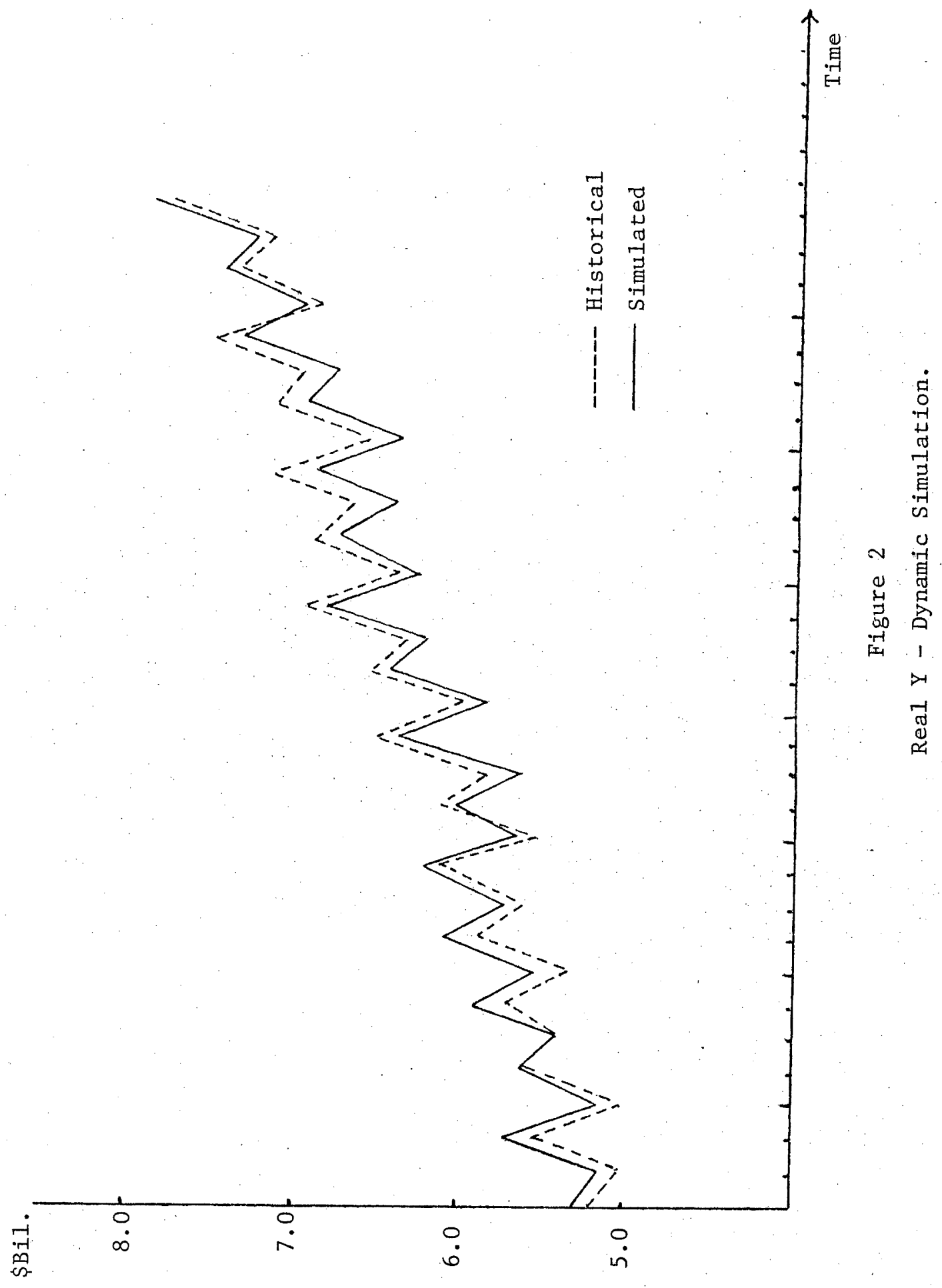




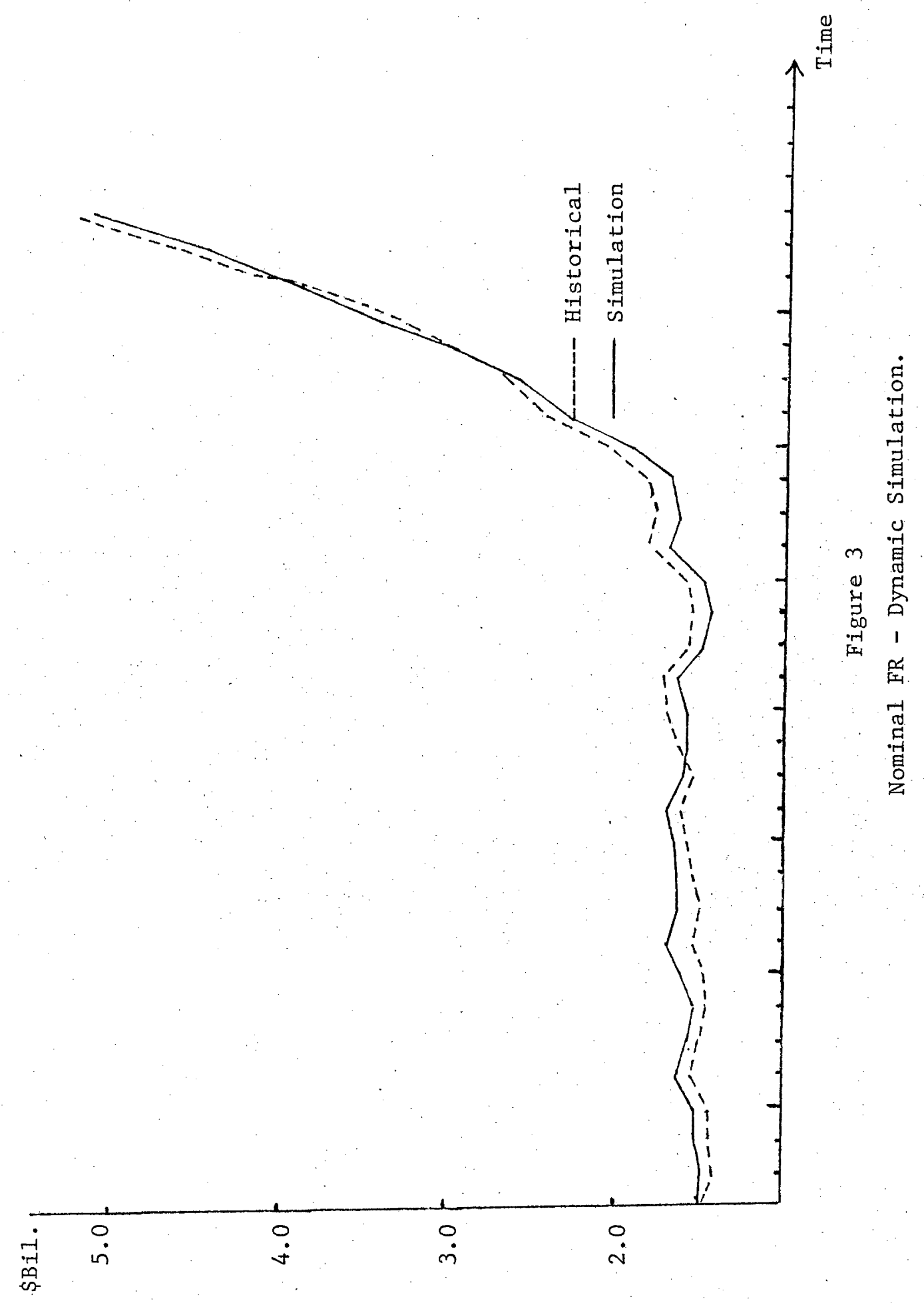




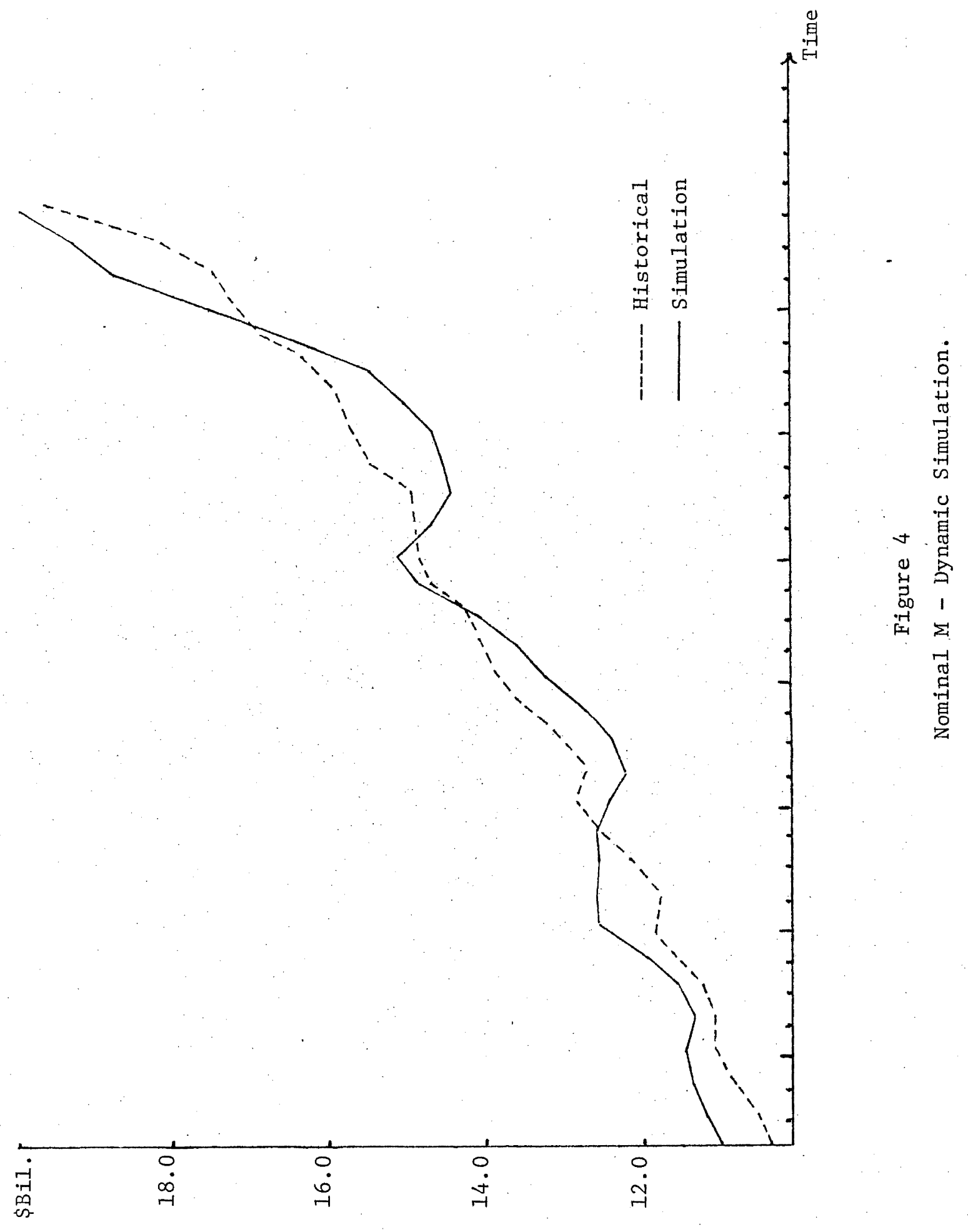




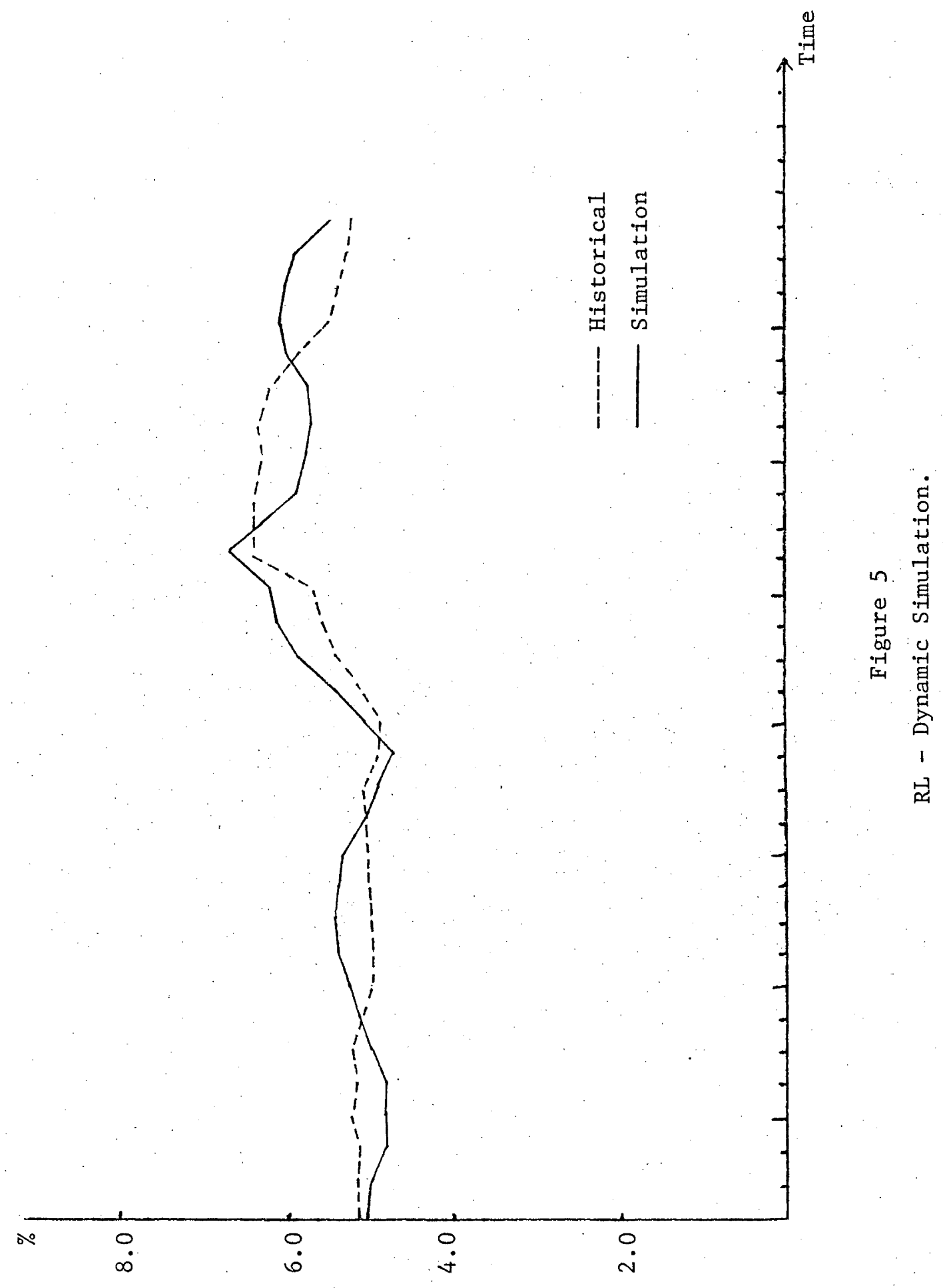


although some reasonably large deviations do occur. (See figure five). The failure of the interest rates and to a lesser extent the failure of the open sector to perform in a manner comparable to income, results in the money supply exhibiting some comparatively large deviations from the historical time series. The impact of this upon the remainder of the system is minimised due to the fact that the money supply coefficients in other equations are very small. As the simulated level of M captures the general behaviour of the historical situation in an adequate manner for most time periods, the overall performance of $M$ is quite acceptable.

Various experiments were carried out to test the response of the system to changes in the controls. As the coefficients of the model all have the correct sign, the direction of the target responses were predictable: The important point to come out of the policy simulation exercises was the fact that an impulse in government spending had a significant effect on income - a not too surprising result which follows directly from conventional multiplier analysis - and at the same time had a substantial effect on the monetary sector and a noticeable effect on the open sector as well. On the other hand monetary policy was considerably less effective in shifting the income variables and required a considerable degree of adjustment to affect any significant response in the open sector due to the manner in which the monetary instrument enters the capital flow equation. These results can be obtained from a close inspection of the structural equations and in fact the simulation exercises convey little additional information than that which can be obtained from such an inspection or by examining the reduced form (see Chapter Five). As a result the full details of the experiments are not presented. The preceding analysis concerning policy responses of the system has important implications for stabilisation as we shall see in 
the following chapters. 
CHAPTER IV

STATE SPACE REALISATION, TARGET SPECIFICATION, COST FUNCTION BIAS AND INSTRUMENT INSTABILITY

The model and stabilisation frameworks set out in the previous two chapters will be implemented to analyse specific policy and target configurations. Before turning to applications, however, some additional framework needs to be developed and discussed. The model as it stands in chapter three is in an unsuitable form for the direct application of the linear/quadratic control laws and the fixed target approaches to stabilisation. To overcome this the model will be converted from the structural form to the reduced form and then placed in its equivalent state space formulation, at the same time clarifying the distinction between the commonly used economic state space form and the engineering state space realisation which is beginning to appear in the economic literature.

The conversion of the structural form to the "true" reduced form is a straightforward procedure and requires no elaboration here. The qualification, "true", refers to the fact that the reduced form obtained is the unique form found from the structure and entirely dependent upon the structure. Any structural change, whether it consists of changing variables within the structure or changing coefficients of variables, will have an effect on the composition of the reduced form. This distinction serves to separate the procedure used here from the Andersen and Carlson (1970) and Andersen and Jordan (1968) concept of an estimated reduced form which ignores the underlying structure and is as we have argued in the previous chapter, an entirely unsuitable method for pursuing macroeconomic stabilisation. The reduced form coefficients for the fifteen endogenous 
variables are set out in Table 1 . The simple simulation experiments carried out in chapter three yielded some insight into possible policy responses of the system. An examination of the reduced form coefficients can achieve the same results but with less precision as the size of the coefficients will depend on how the data was scaled for the estimation of the model. Note that both instruments have a contemporaneous impact on all endogenous variables. In terms of the fixed target approach this implies that as long as the number of targets equals the number of instruments (in this case two) policy planners can hit any configuration of independent targets without any anticipation. The contemporaneous impact is derived from the fact that within the underlying structure, income, $Y$, has a contemporaneous influence on every endogenous variable. and as government spending constitutes an important component of the national income identity, it too has an impact on all endogenous variables. The effect of monetary policy is less direct as it only operates directly on two equations, money supply and capital flows, which in turn feed back into additional equations. An indirect effect operates through the long rate of interest which does not have any effect on the income, monetary and open sectors until a four-period lag has transpired. This indirect effect of monetary policy is reflected in the fact that the variable $\mathrm{RL}_{t-1}$ only has an impact on the long rate of interest in the very short run. The fact that the reduced form exhibits contemporaneous impacts from all instruments has an important consequence for the formulation of optimal stabilisation policies. Pindyck (1973a) (1973b) found that when a particular policy variable only operated with an $n$ period lag on most endogenous variables, with the end of a particular planning period only $n$ periods away or some number less than $n$, the optimal solution for both targets and instruments would behave in a 


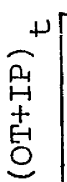

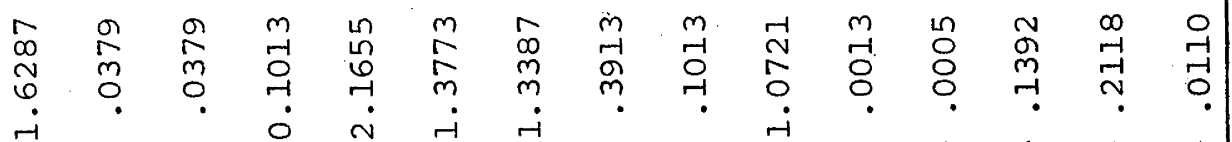

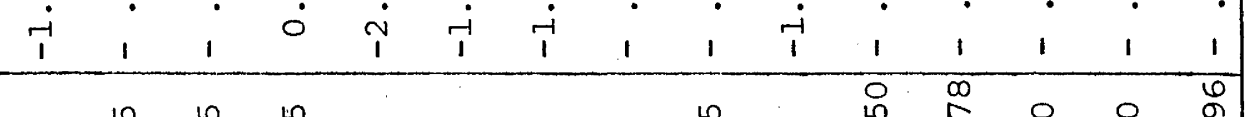

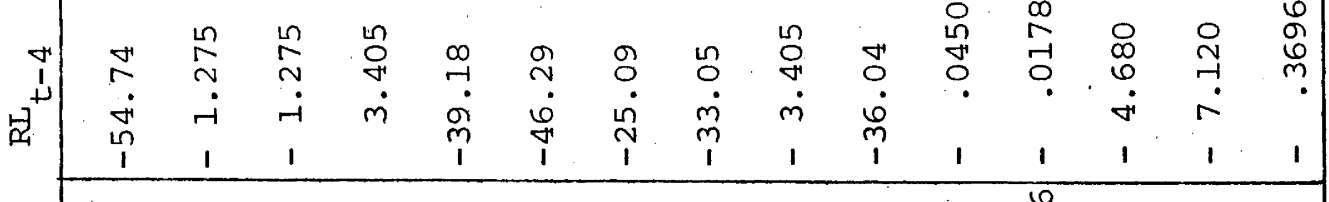

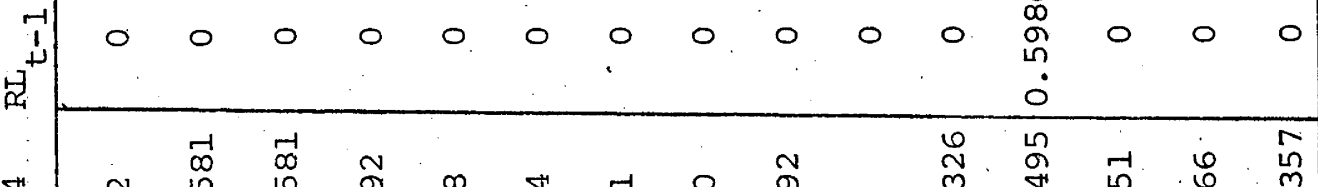

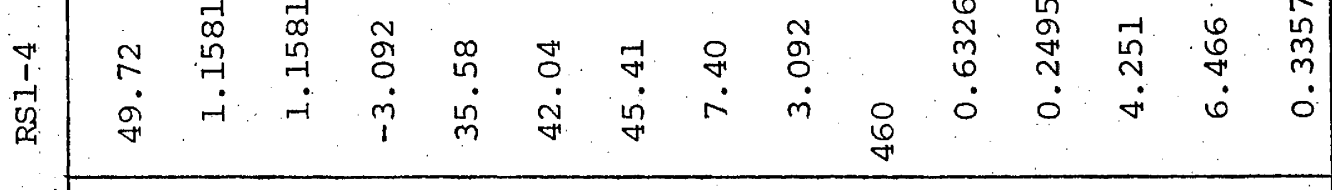

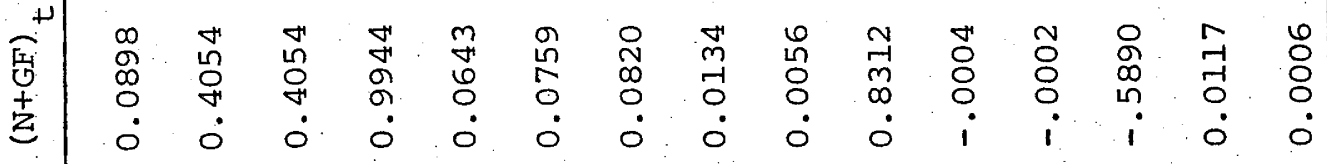

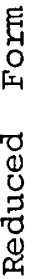

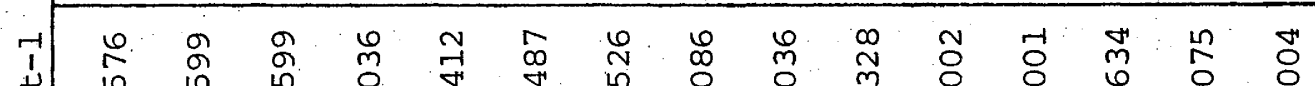

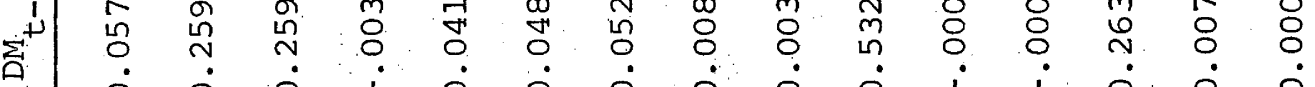

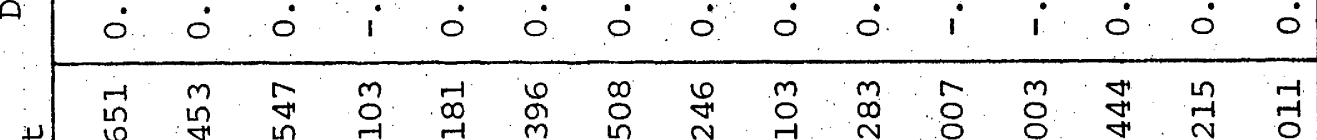

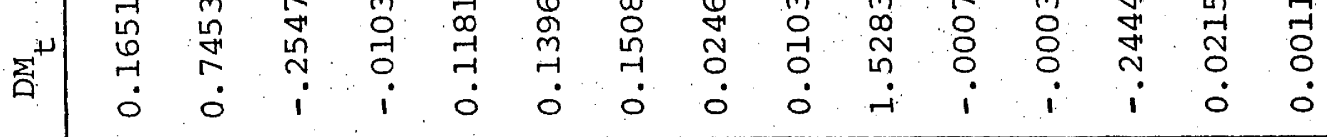

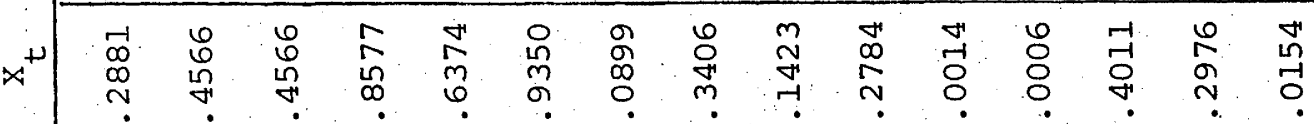

$\underset{\substack{0 \\-1}}{-1}$

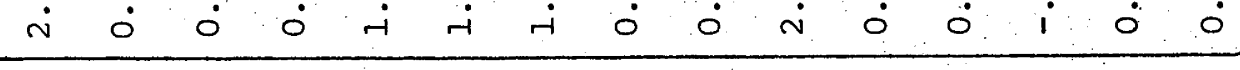

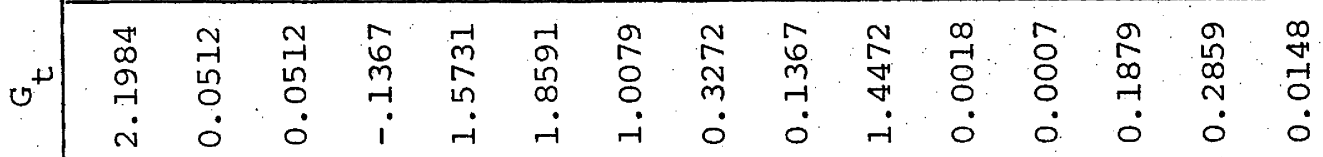
$\begin{array}{lllllllllll}-1 & -1 & 0 & 0 & -1 & 0 & 0 & 0 & 0 & 0\end{array}$

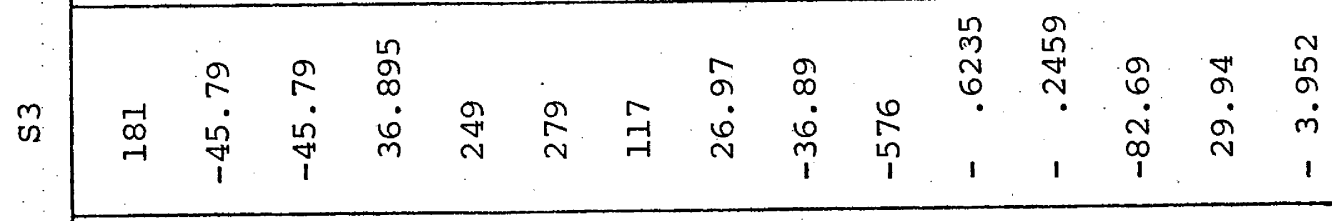

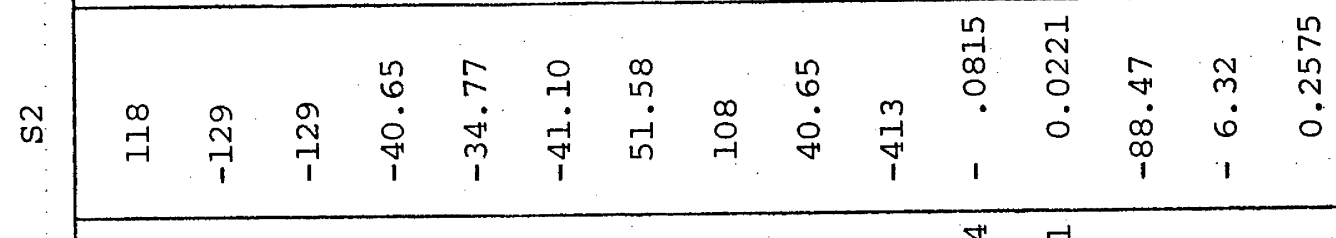

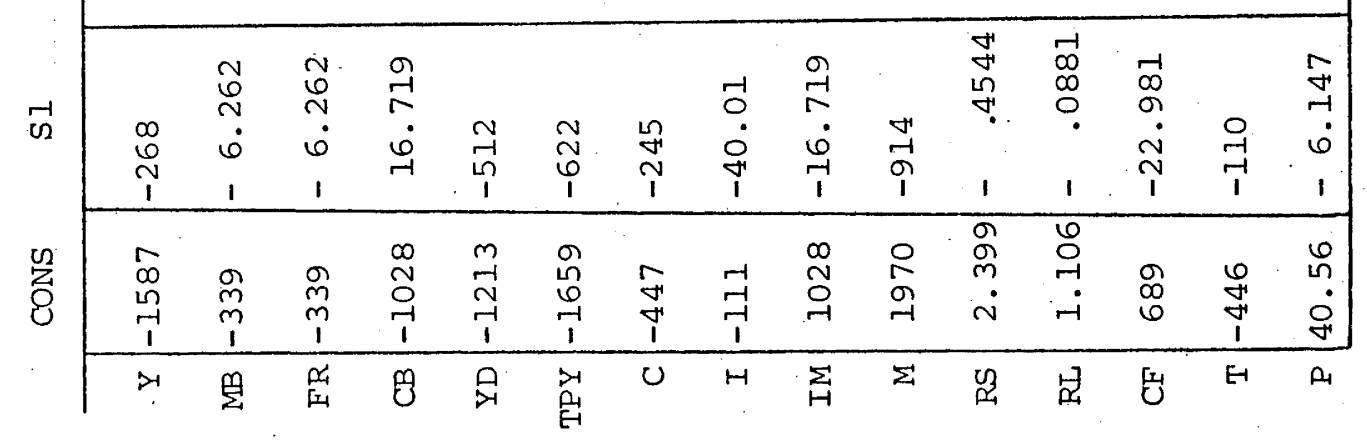




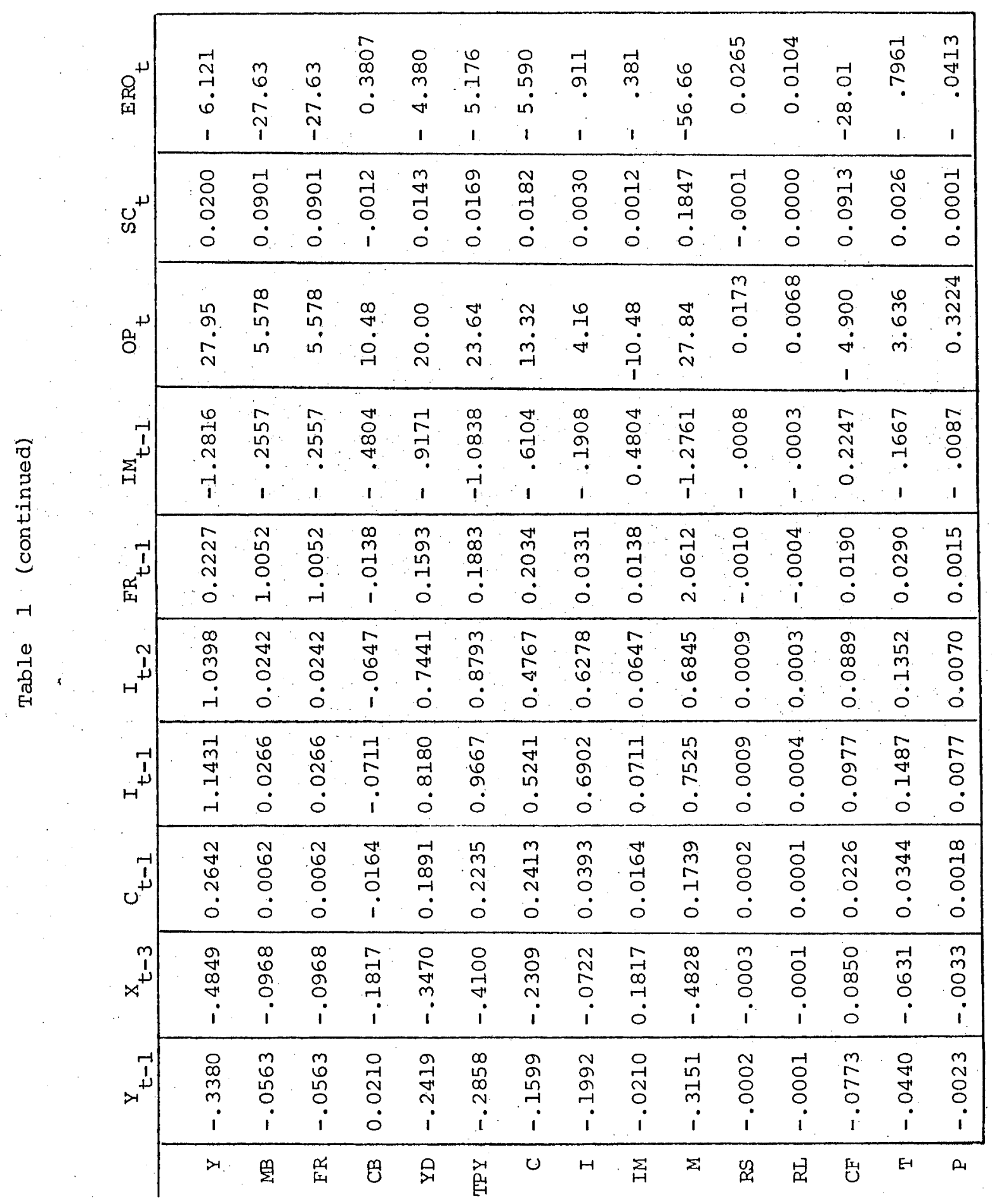


peculiar fashion. If the end of the planning period was $n$ periods away and if monetary policy had an impact lag of $n$ periods then it is clear that monetary policy will equal its target level (provided some positive weight is applied to deviations of the instrument from its desired level) over those $n$ periods as it cannot contribute to the solution. In effect then problems could arise in the next planning period due to the passive nature of monetary policy. The reason why Pindyck obtained this solution is that he failed to specify any financial wealth effects on consumption as have been incorporated into the model presented here. The impact effect of all instruments ensures that an instrument will not become passive (specifically not directed towards any contemporaneous targets) over the final periods of the planning period which gives the strange behaviour obtained by Pindyck. The removal of this problem ensures that one complicating factor in the choice of the length of the planning period has been removed. That is, it will not be necessary to extend a desired planning period by $n$ periods to ensure that all instruments are active over the desired time-span.

While the reduced form and simulations are useful in gaining insight into policy responses, it is usually impossible to ascertain a priom what effect the choice of a particular target configuration will have on the remainder of the endogenous variables and the instruments. It is clear from Table 1 that the degree of effectiveness of both monetary and fiscal policy is limited but this in itself is not sufficient to indicate whether or not a particular instrument should be aimed at a particular target. Consider the reduced form instrument coefficients for Y. G has an impact of 2.2 while DM only has an impact of 0.165 . If it was desired to direct monetary policy towards the income target, and purely on the basis of the reduced form there is no reason why this should 
not be the case, then it would be reasonable to expect that monetary policy would need to be used in a more extensive manner than the use of fiscal policy in a similar situation (given that a similar scaling procedure for $G$ and $D M$ was used). It is not immediately clear what would happen to the remainder of the system. A similar case could be stated if fiscal policy was directed towards say the foreign reserves target where clearly fiscal policy is limited in effectiveness compared with monetary policy. A situation such as that outlined above could result under an ad hoc type approach to stabilisation and result in destabilising the rest of the system as well as resorting to severe and undesirable movements in the time paths of the instruments. The fact that some endogenous variables are influenced in various degrees of effectiveness, directly or indirectly through other endogenous variables, indicates the difficulties that could arise in obtaining the appropriate policy mix to achieve a given set of targets. A large number of simulations may produce the desired mix but the cost in money terms and time would be prohibitive. Should fiscal policy be employed solely to adjust expenditure variables, thus leaving monetary policy to offset the impact of fiscal policy on other sectors and at the same time to try and move the monetary and open sectors towards specified targets? Or should both instruments be used to complement each other? These questions are difficult to answer by studying the reduced form coefficients. As we shall see, by incorporating policy goals into a formal optimisation framework direct information can be obtained as to the overall effectiveness and the appropriate mix of monetary and fiscal policies. In particular, it will become clear that the linear quadratic solution will produce a complementary mix of policy which takes into account the relative effectiveness of each policy on each particular target variable. 
The information contained within the reduced form will be utilised to its fullest extent in obtaining the appropriate mix and once the $\mathrm{mix}$ is obtained it is constructive to return to the reduced form to gain some further insight into why a particular policy has been designated.

The above section has argued that while the reduced form may contain all the information required to successfully pursue stabilisation policies, it is not an easy matter to effectively use that information as so many policy effects and dynamic effects are continuously feeding through the system. The reduced form is then viewed as a tool for obtaining more precise information about possible policy options which can be carried out in a linear/quadratic or dynamic fixed targets framework. Before any analysis can be carried out however, the reduced form must be converted to its equivalent state space (one period lag) form. In converting the system to its state space form we shall make several appropriate observations on the alternative procedures that can be adopted. The economic literature has diverted from the control engineering literature in respect to state-space formulations of systems of difference equations. The economic literature has focussed on what shall be called the Chow-Turnovsky formulation (see Chow (1973), (1975a) and Turnovsky (1974)). This is not to say that these writers have been credited with the formulation of the procedure but the techniques set out in the abovementioned papers have come to be accepted by economists as the standard type of procedure to be used. The discussion of the dynamic fixed targets approach to stabilisation set out in Chapter Two utilises the control engineering approach to state space formulation with which economists have less familiarity (a recent high level text book contribution to the economic literature by Aoki (1976) mentions the engineering approach but does not explain its derivation). The Chow- 
Turnovsky state space realisation is derived from the reduced form in the following manner. The reduced form of an economic system may be written as

$$
\begin{aligned}
Y_{t}= & A_{1} Y_{t-1}+A_{2} Y_{t-2}+\ldots+A_{m} Y_{t-m}+C_{0} u_{t}+C_{1} u_{t-1}+\ldots \\
& +C_{n} u_{t-n}+D_{0} z_{t}+D_{1} z_{t-1}+\ldots+D_{k} z_{t-k}+a_{0}+\varepsilon_{t}
\end{aligned}
$$

where the $A_{i}, C_{i}, D_{i}$ are equivalent to constant matrices of coefficients of lagged endogenous variables and constant matrices of lagged and current control and exogenous variables respectively. As in Chapter two, $\mathrm{Y}_{t}$ and $\mathrm{Y}_{t-i}$ are vectors of current and lagged endogenous variables while $u_{t-i}$ and $z_{t-i}$ are vectors of current and lagged control and exogenous variables (for contemporaneous effects $i=0$ ).. $G_{0}$ and $\varepsilon_{t}$ maintain the definitions of Chapter two. Note that the reduced form of the model could be left in the form of (4.1) but this would invalidate the general applicability of the controls already derived and would unnecessarily complicate the derivation of suitable control laws and the subsequent numerical computation of optimal control and target paths. For example, if there are lags greater than one period in the endogenous variables of the reduced form of an econometric model the appropriate linear feedback control equation may be given by

$$
u_{t}^{*}=F_{1 t^{y}-1}+F_{2 t} y_{t-2}+F_{3 t} y_{t-3} \cdots+f_{t}
$$

thus precluding the efficient recursive approach to the solution set out in Chapter two. The use of a state space realisation is not necessary to compute optimal stabilisation policies but it does substantially facilitate computation.

The reduction of (4.1) to a first order system is carried out by defining new variables to replace endogenous, control and exogenous 
variables which have lags greater than one period. That is, these variables are mapped into a vector of current states which will consist not only of the current endogenous variables of the system but also of the newly defined, or auxiliary, variables. In terms of (4.1) the following new variables can be defined:

$$
\begin{aligned}
& y l_{t}=y_{t-1}, y 2_{t}=y l_{t-1}=y_{t 2}, \ldots . y(m-1)_{t}=y(m-2)_{t-1}=y_{t-m+1} \\
& u l_{t}=u_{t-1}, u 2_{t}=u l_{t-1}=u_{t-2}, \ldots u(n-1)_{t}=u(n-2)_{t-1}=u_{t-n+1} \\
& z l_{t}=z_{t-1}, z 2_{t}=z l_{t-1}=z_{t-2}, \cdots \cdot z(k-1)=z(k-2)_{t}=z_{t-k+1}
\end{aligned}
$$

Using (4.3), (4.4) and (4.5), (4.1) can be rewritten as

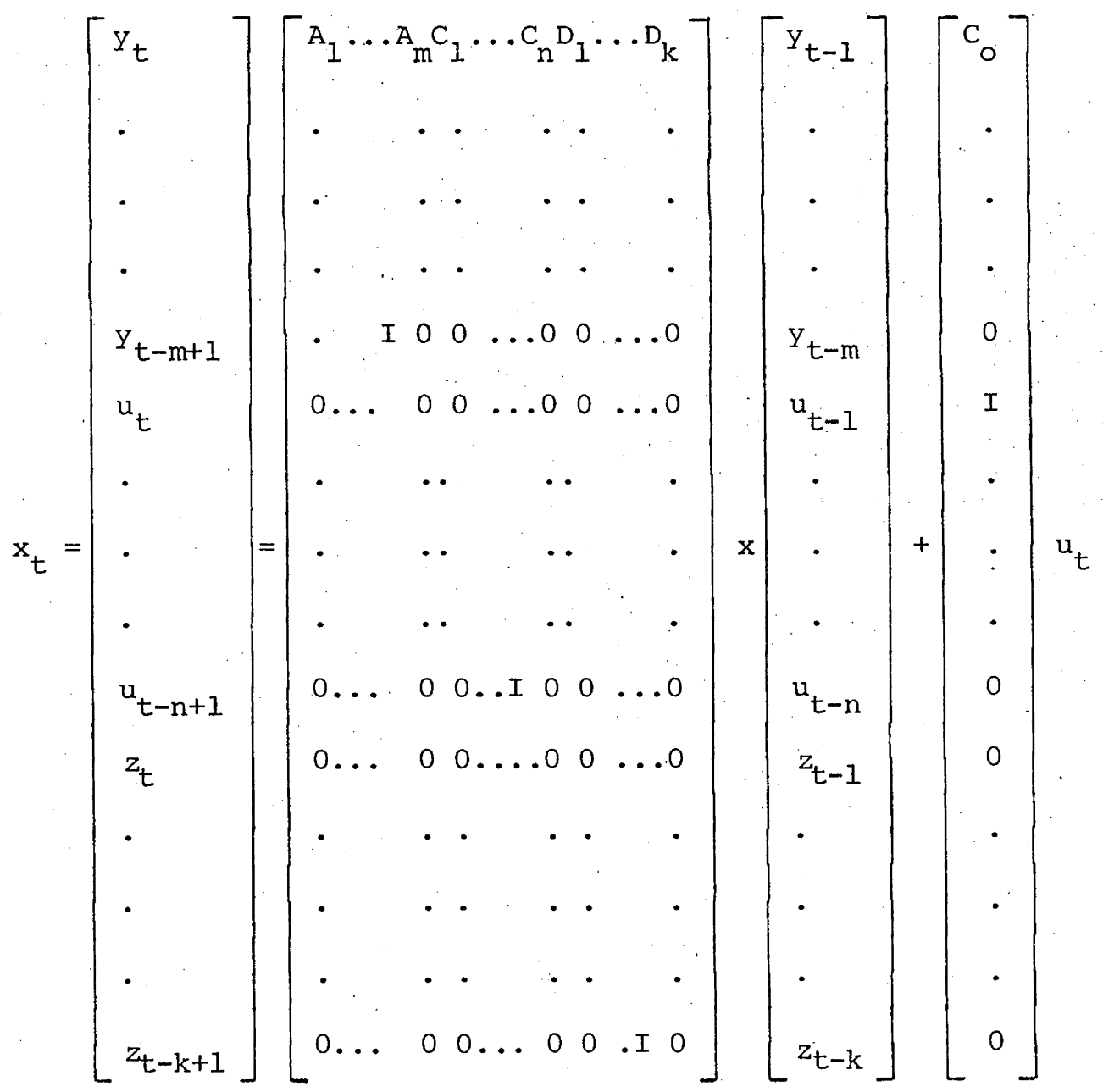




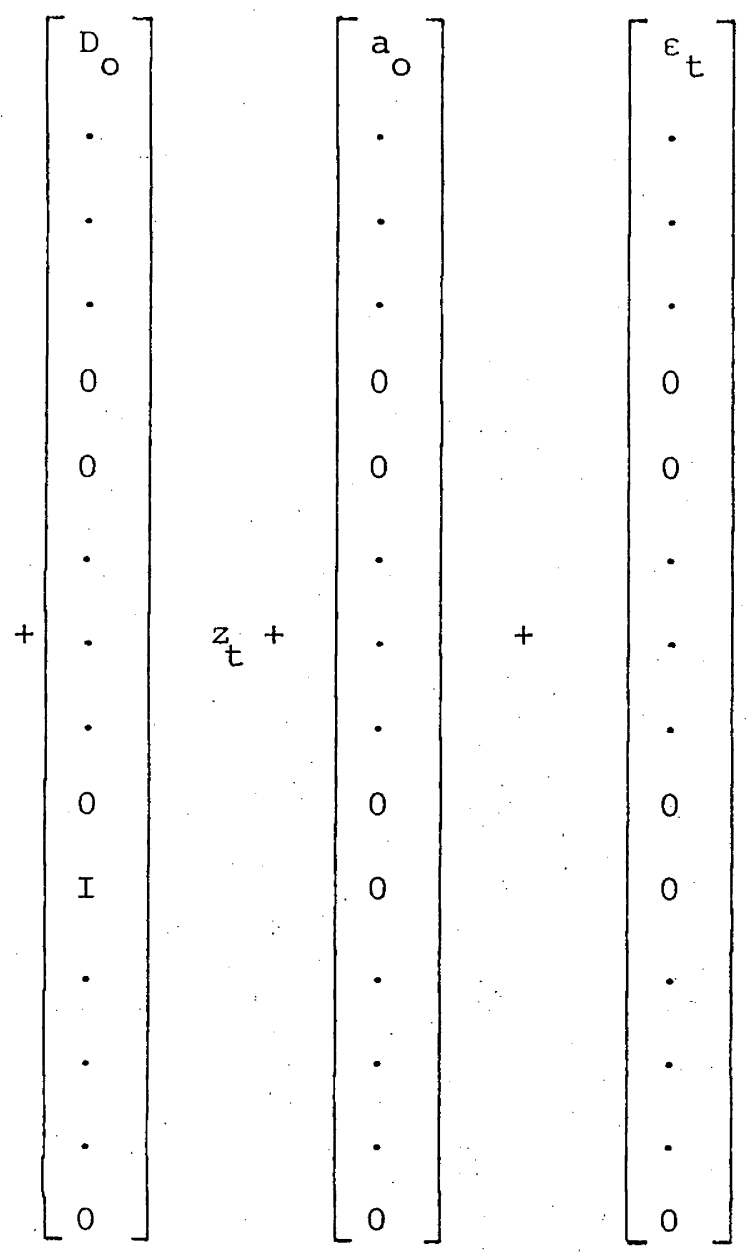

where $x_{t}$ is designated to be the new vector of state variables. (4.6) represents a mapping of the original reduced form, (1.4), into a system of the following form

$$
x_{t}=A x_{t-1}+C u_{t}+b_{t}+\varepsilon_{t}
$$

where

$$
b_{t}=D z_{t}+a_{0}
$$

which of course is identical to the system (2.5) which was used to derive the relevant optimal control laws. Before commenting on the desirable and undesirable features of (4.6) it is convenient to reintroduce the control engineering state space realisation used to derive the approach to the solution of the fixed target anticipation problem. Recall that the policy model of Chapter two could be written as

$$
x_{t}=\hat{F} x_{t-1}+\hat{G} u_{t-1}+E z_{t-1}
$$




$$
\mathrm{Y}_{t-1}=P \mathrm{x}_{t-1}+\hat{S u}_{t-1}+M z_{t-1}
$$

where $F$ and $G$ are written with a hat to avoid confusion with the notation employed in the optimal control laws. The use of (4.9) and (4.10) as a state space representation necessitates an alternative mapping procedure to that found in the derivation of (4.6). Consider the following simple example where the exogenous variables have been eliminated for ease of exposition. The reduced form for the example is given by

$$
\begin{aligned}
Y_{t}= & A_{1} y_{t-1}+A_{2} Y_{t-2}+A_{3} t_{t-3}+C_{0} u_{t}+C_{1} u_{t-1} \\
& +C_{2} u_{t-2}+C_{3} u_{t-3}
\end{aligned}
$$

Subtracting $\mathrm{C}_{0} \mathrm{u}_{t}$ from both sides gives

$$
\begin{gathered}
y_{t}-C_{0} u_{t}=A_{1} y_{t-1}+A_{2} y_{t-2}+A_{3} y_{t-3}+C_{1} u_{t-1} \\
+C_{2} u_{t-2}+C_{3} u_{t-3}
\end{gathered}
$$

Finally, the right hand side of (4.12) has the expression $\mathrm{A}_{1} \mathrm{C}_{0} \mathrm{u}_{t-1}$ added and subtracted to maintain equality. Note that the expression $\mathrm{C}_{0} \mathrm{u}_{t-1}$ refers to the impact of the controls on $\mathrm{y}_{t-1}$ one period before and should not be confused with $c_{1} u_{t-1}$ which refers to the impact of last periods controls on the current vector of endogenous variables.: The new system can be written as

$$
\begin{gathered}
y_{t}-C_{0} u_{t}=A_{1}\left(y_{t-1}-C_{0} u_{t-1}\right)+A_{2} Y_{t-2}+A_{3} t_{t-3} \\
+\left(C_{1}+A_{1} C_{0}\right) u_{t-1}+C_{2} u_{t-2}+C_{3} u_{t-3}
\end{gathered}
$$

To put (4.13) into the same form as (4.9) and (4.10) the following state variables can be defined 


$$
\begin{aligned}
& z 1_{t}=y_{t}-C_{0} u_{t} \\
& z 2_{t}=y_{t-1} \\
& z 3_{t}=y_{t-2} \\
& z 4_{t}=u_{t-1} \\
& z 5_{t}=u_{t-2}
\end{aligned}
$$

Having derived two different state space realisations the question needs to be asked of which one should be chosen to formulate the model of Chapter Three in? First of all note that in the engineering procedure only five state variables would be required whereas the economic realisation of (4.6) would require six state variables for the same reduced form. For very large systems the reduction in the size of the system may be important in terms of computer storage. On the debit side, the engineering approach requires an alternative composition of the state vector in that the variable of prime concern, $y_{t^{\prime}}$ does not explicitly appear by itself but only as a composite term, $y_{t}-C_{0} u_{t}$. This formulation would be clumsy in computing optimal policies in the linear/ quadratic framework and some modifications to the control procedure would be necessary as well as the fact that by obscuring $y_{t^{\prime}}$ some of the intuitive insight into the behaviour of linear feedback control laws and the corresponding Ricatti equations may be lost, especially to the non expert. The situation is as follows. The optimal control laws have been derived using the conventional Chow-Turnovsky state space realisation which, while being a system of greater dimension than the corresponding engineering realisation, gives a clear insight into the behaviour of systems under optimal control as the underlying reduced form is maintained throughout. On the other hand, following the recent 
literature of Preston-Sieper (1977) the dynamic fixed target approach is formulated in control engineering terms. Can the two be reconciled? The answer is obviously yes when it is realised that the two alternative methods are interchargeable, that is, they are alternative ways of expressing the same model with one being more efficient in terms of system size requirements than the other. In fact, system (4.6) can be written in the same form as (4.9) and (4.10) as (ignoring the exogenous variables)

$$
\begin{aligned}
& x_{t}=\bar{F} x_{t-1}+\bar{G} u_{t} \\
& y_{t-1}=P x_{t-1} .
\end{aligned}
$$

The only difference in the general form from (4.9) and (4.10) being that the controls appear with the same time subscript as the state vector $\mathrm{x}_{t}$ and $x_{t}$ explicitly includes $y_{t}$ as the first component. Also note that as the solution to the fixed target problem is co-ordinate free, then any method of calculating the dynamic multipliers is admissible so long as the solution is consistent with the specification of the state space. Furthermore, if the Chow-Turnovsky realisation is expressed in the form of (4.15) and (4.16) then using the procedure outlined in Chapter two, the dynamic instrument multipliers for that system can be written as

$$
\pi_{\mathrm{V}}=\mathrm{P} \overrightarrow{\mathrm{F}}^{\mathrm{V}} \overline{\mathrm{G}}
$$

The above discussion has shown that the two approaches to the state space realisation are conceptually different in the basic approach of each but are just different ways of expressing the dynamic structure of an economic model. The choice of which representation to use is dependent upon the preferences of the researcher and the uses to which it is to be put. The state space technique employed in this study is a slight variant on the Chow-Turnovsky framework. This method was chosen 
to enable an effective computer package (which was one of the secondary aims of this study) to be developed which would solve both optimal flexible target problems and fixed target problems. The choice of the Chow-Turnovsky framework enables any linear model to be fed into the computer, which is in the appropriate form, by economists not generally familiar with the engineering literature. The co-ordinate free nature of the fixed target approach and the relationship between the ChowTurnovsky specification and the dynamic multipliers given by (4.17) means that the same specification can be used to efficiently solve both problems. Even though the engineering approach has been rejected, it is nonetheless very useful to know how it is formulated (equations (4.11) to (4.14)) as exposition of this has not readily filtered into accepted economic usage which makes reading of some of the latest literature (for example Preston and Sieper (1977) and Preston (1977)) extremely difficult for the non-specialist, and even difficult for the economic control theorist who has only acquired familiarity with the ChowTurnovsky framework.

The state space representation of the model contained within Chapter Three and which appears in the pages that follow is a slight variant on that given by (4.6). If the mapping of (4.6) is followed literally then all controls and exogenous variables would be mapped into the state vector. The framework for optimal stabilisation presented by Chow in various papers requires that the controls must be included in the state vector. This approach is avoided here and is one reason why the controls are explicitly included in the cost function separately from the states. The mapping of the controls into the state vector needlessly expands the dimensions of the system and for very large systems could create severe computer storage problems. The modification to (4.6) will 
be that only those variables with lags greater than one period will be incorporated into the state vector, with the exception of the lagged monetary instrument, $\mathrm{DM}_{t-1}$ ' which will also be mapped into the state vector. The new variables and their definitional equations are given below.

$$
\begin{aligned}
& R L I_{t}=R L_{t-1} \\
& R L 2_{t}=R I_{t-1}=R L_{t-2} \\
& R L 3_{t}=R L 2_{t-1}=R L_{t-3} \\
& R S 1_{t}=R S_{t-1} \\
& R S 2_{t}=R S I_{t-1}=R S_{t-2} \\
& R S 3_{t}=R S 2_{t-1}=R S_{t-3} \\
& I 1_{t}=I_{t-1} \\
& S M_{t}=D_{t} \\
& S X_{t}=x_{t} \\
& S X I_{t}=S X_{t-1} \\
& S X 2{ }_{t}=S X I_{t-1}=S X_{t-2}
\end{aligned}
$$

The variable $\mathrm{SM}_{t}$ is defined to be equal to $\mathrm{DM}_{t}$ to avoid confusion and takes account of the lagged value of the domestic monetary base in the capital flow equation. $\mathrm{SM}_{t}$ will appear in the state vector, $\mathrm{x}_{t}$, but will be treated as an auxiliary variable and hence will not be assigned a target or be associated with control costs. By definition it will be equal to the value of the optimal monetary policy generated by $(2 ; 31)$. In the new system matrix, $\mathrm{A}$, of (4.7), the appropriate coefficients will be equated with $S_{t-1}$ as designated in the reduced form. With the eleven newly defined variables, the state vector $x_{t}$ will consist of 
twenty-six variables giving the system a state dimension of $26 \times 26$. The matrix C will have a dimension of $26 \times 2$ and D will be $26 \times 9$. Recall that in the original structural form of the model there are eleven exogenous variables. However, it is convenient to aggregate NT and GF and OT with IP (see the reduced form) thus reducing the dimensions of the system. As a point of comparison between the state space realisation used here and the engineering formulation, if the latter had been used there would not have been any need to map $\mathrm{DM}_{t-1}$ into the state vector. The state space form of the model is a crucial component of the study as it can be immediately used to analyse any flexible target problem and can be manipulated to derive the dynamic multipliers of the system in order to analyse any fixed target controllability problem. The relevant matrices are presented below. The state vector is given by

$$
x_{t}=\left[\begin{array}{c}
Y \\
M B \\
F R \\
C B \\
Y D \\
T P Y \\
C \\
I \\
I M \\
R S \\
R L \\
C F \\
T \\
P \\
R L I
\end{array}\right]
$$




$\left.\mid \begin{array}{c}R L 1 \\ R L 2 \\ R L 3 \\ R S 1 \\ R S 2 \\ R S 3 \\ S M \\ I 1 \\ S X \\ S X 1 \\ S X 2\end{array}\right]$

\section{TARGET SPECIFICATION}

The specification of targets or desired paths for both

endogenous and control variables presents some difficulty as the choice of targets will necessarily affect the results obtained from either a flexible or fixed target approach to stabilisation. Studies in the past have tended to gloss over this problem and treat the problem of target specification in a simplified manner. The typical approach is to construct the desired paths for both endogenous and control variables as simple linear mappings of the initial conditions into a target vector. This approach would perhaps be satisfactory for examining the nature of policy over an historical time span but may not be satisfactory for an analysis and planning of policy when the economy is on an upswing or downswing for example. Consider a situation in which it is desired to gain insight into appropriate policies to move out of a recession. If the model was estimated over a recessionary period then the sluggish behaviour of the economy would be reflected in the structure and even if 
$\mathrm{Ax}_{t-1}$

\begin{tabular}{|c|c|c|c|c|c|c|}
\hline & -.3330 & .0000 & .2227 & .0000 & .0000 & .0000 \\
\hline & -.0563 & .unon & $1.005 ?$ & .0000 & .10000 & .0000 \\
\hline & -.0563 & .0000 & 1.0052 & . & .0000 & $.000 n$ \\
\hline & .0210 & .0000 & -.0138 & .0000 & .0000 & .0000 \\
\hline & -.2419 & . & .1593 & .0000 & .0000 & .0000 \\
\hline & -.2858 & .0000 & .1883 & .0000 & .0000 & .0000 \\
\hline & -.1599 & . & .2034 & .0000 & .0000 & .0000 \\
\hline & -.1992 & $.00 n 0$ & .0331 & .0000 & .0000 & .0000 \\
\hline & -.0210 & .0000 & .0139 & .0000 & .0000 & .0000 \\
\hline & $=.3151$ & $.000 n$ & 2.0612 & -0000 & .0000 & .0000 \\
\hline & -.0002 & . onon & -.0010 & .0000 & .0000 & .0000 \\
\hline & -.0001 & .0000 & -.0004 & .0000 & .0000 & .0000 \\
\hline & -.0773 & .0000 & .0190 & .0000 & .0000 & .0000 \\
\hline - & $=.0440$ & חסבים. & .0290 & .0000 & .2000 & .000.0. \\
\hline & -.0023 & .0000 & .0015 & .0000 & .0000 & $.000 n$ \\
\hline$\ldots$ & .0000 & .0000 & .0000 & .0000 & .0000 & .0000 \\
\hline & .0000 & .0000 & .0000 & .0000 & .0000 & . onon \\
\hline - & - . vona & 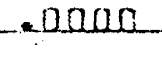 & . & . & . & תנחתמ- \\
\hline & ס000 & .0000 & .0000 & 1.0000 & .0000 & .0000 \\
\hline- & $\because 0000$ & . 0000 & - & .0000 & .0000 & - conan. \\
\hline & .0000 & .0000 & .0000 & .0000 & .0000 & .0000 \\
\hline & | & . & مصمص. & .0200 & - 10000 & סمD0. \\
\hline & .0000 & .0000 & .0000 & .6000 & .0000 & .0000 \\
\hline … & .0000 & . ouno & $.000 \Omega$ & .0200 & $.0 D C D$ & .0000 \\
\hline & .0000 & .0000 & .0000 & $.0 n 00$ & .0000 & .0000 \\
\hline & & . & . & .0000 & .0000 & .0000 \\
\hline
\end{tabular}


$\Delta x_{t \cdots 1}$ continued.

\begin{tabular}{|c|c|c|c|c|c|}
\hline $.264 ?$ & 1.1431 & -1.2816 & .0700 & 49.7175 & .0000 \\
\hline .0062 & .0266 & -.2557 & .0000 & 1.1581 & .12000 \\
\hline .0062 & .0266 & -.2557 & .0000 & 1.1581 & .0000 \\
\hline-.0164 & -.0711 & -.4804 & .0000 & -3.0924 & .0000 \\
\hline .1891 & .8180 & -.9171 & .0000 & 35.5777 & .0000 \\
\hline .2235 & .9667 & -1.0838 & .0000 & 42.0441 & .0000 \\
\hline .2413 & .5241 & $-: 6104$ & .0000 & 45.4092 & .0000 \\
\hline .0393 & .6902 & -.1909 & .0000 & 7.4007 & .0000 \\
\hline .0164 & .0711 & .4804 & .0000 & 3.0924 & .0000. \\
\hline .1739 & .7525 & -1.2761 & .8000 & 460.2491 & .0000 \\
\hline .0002 & .0009 & -.0008 & .0000 & .6326 & .0000 \\
\hline .0001 & $.0 \cap 04$ & -.0003 & .0000 & .2495 & .5986 \\
\hline .0226 & .0977 & .2247 & .0000 & 4.2505 & .0000 \\
\hline-.0344 & .1482 & -.1667 & .0000 & 6.4664 & .0707 \\
\hline .0018 & .0077 & -.0087 & .0000 & .3357 & .0000 \\
\hline .0000 & .0000 & .0000 & .0000 & .0000 & 1.0000 \\
\hline .0000 & $.0 n 00$ & .0000 & .0000 & .0000 & $.000 \%$ \\
\hline-.0000 & . & .0000 & מחסח. & .0000 & . \\
\hline .0000 & .0000 & .0000 & .0000 & 1.0000 & .0000 \\
\hline .0000 & .0000 & .0002 & .0000 & .0000 & مع0.0. \\
\hline .0000 & .0000 & .0000 & .0000 & .0000 & $.000 n$ \\
\hline - & .0000 & - & .0000 & .0000 & مan. \\
\hline .0000 & 1.0000 & .0000 & .0000 & .0000 & .0000 \\
\hline .0000 & .0000 & .0000 & .0000 & .0000 & .0002 \\
\hline .0000 & .0000 & .0000 & .00013 & .0000 & . \\
\hline -.0000 & - 0000 & -.0000 & -.0000 & .0000 & .0000 \\
\hline
\end{tabular}


$\mathrm{Ax}, \ldots \quad$ continued.

\begin{tabular}{|c|c|c|c|c|c|}
\hline .0000 & חס & .0000 & . & .0000 & -54.7449 \\
\hline .0000 & .0000 & .00000 & .0000 & $.000 a$ & -1.2752 \\
\hline .0000 & .0000 & .0000 & .0000 & .0000 & $-1.275 ?$ \\
\hline $.000 \pi$ & .0000 & .0000 & .0000 & .0000 & 3.4051 \\
\hline .0000 & .0000 & .0000 & ססחס. & .0000 & -39.1753 \\
\hline .0000 & .0000 & $.000 \pi$ & .0000 & .0000 & $-46 \cdot 2955$ \\
\hline .0000 & .0000 & .0000 & .0000 & .0000 & -25.0982 \\
\hline .0000 & .0000 & .0000 & .0000 & .0000 & -33.0519 \\
\hline ב000 & .0000 & .0000 & .0000 & .0000 & -3.4051 \\
\hline .0000 & -.0000 & .0000 & .0000 & .0000 & -36.01383 \\
\hline .0000 & .0000 & - croú & .0000 & .0000 & -.0450 \\
\hline .0000 & .0000 & .0000 & .0000 & .0000 & -.0178 \\
\hline .0000 & .01100 & .0000 & .0000 & .0000 & -4.6203 \\
\hline ملمan. & - & . & .0000 & .0000 & -7.1203 \\
\hline .0000 & .0000 & .0000 & .0000 & .0000 & -.3696 \\
\hline .0000 & . adar & .0000 & -. & $.000 a$ & . Doder \\
\hline .0000 & .0000 & .0000 & $1.0 n 00$ & .0000 & .0000 \\
\hline .0000 & .00000 & - conon & -.0000 & 1.0.0م & $.000 \mathrm{C}$ \\
\hline .0000 & - (10000 & .0000 & .0000 & .0000 & .0000 \\
\hline .0000 & .0000 & .0000 & .0000 & .0000 & conc \\
\hline .0000 & .0000 & .0000 & - 0 noo & ס000 & .0000 \\
\hline - & .0000 & -.0000 & .0000 &. DCoO & .0000 \\
\hline .0000 & .0000 & .0000 & $.00 n 0$ & .0000 & .0000 \\
\hline .0000 & .0000 & - conan & .0000 & .0000 & .0000 \\
\hline .0000 & $.0 n a n$ & .0000 & .0000 & .0000 & ח.000 \\
\hline مסחم.- & חمaم. & . & .0.0. & .0000 & $-.0 u a n$ \\
\hline
\end{tabular}


$\mathrm{Ax}_{t-1 \quad \text { continued }}$

\begin{tabular}{|c|c|c|c|c|c|}
\hline .0000 & .0000. & -49.7175 & .0576 & 1.0398 & .0000 \\
\hline .0000 & $.00 n \pi$ & -1.1581 & .2599 & .0242 & .0000 \\
\hline .0000 & .0000 & -1.1581 & .2599 & .0242 & .0000 \\
\hline .0000 & .0000 & 3.0924 & -.0036 & $=.0647$ & .0000 \\
\hline $.000 n$ & .0000 & -35.5777 & .0412 & .7441 & .0000 \\
\hline .0000 & .0000 & $-42.044 I$ & .0487 & .8793 & $.0 n a n$ \\
\hline .0000 & .0000 & -45.4092 & .0526 & .4767 & .0000 \\
\hline .0000 & $.000 n$ & $-7.400 ?$ & .0086 & .6278 & .0000 \\
\hline .0000 & .0000 & -3.0924 & .0036 & .0647 & .0000 \\
\hline .0000 & .0000 & -460.2491 & .5328 & -6.845 & - anan \\
\hline .0000 & - vudar & -.6326 & $-.000 ?$ & .0009 & .0000 \\
\hline .0000 & .00010 & -.2495 & -.0001 & .0003 & $.0 n 0 n$ \\
\hline $.000 n$ & .0000 & $-4 \cdot 2505$ & .2634 & .0889 & .0000 \\
\hline مصمn. & .0000. & $=6.4664$ & .0075 & $.1 \geq 52$ & م0م0.. \\
\hline .0000 & .0000 & -.3357 & .0004 & .0070 & .0000 \\
\hline $.000 \Omega$ & .0000 & .000 & .0000 & .0000 & .000 .0 \\
\hline .0000 & .0000 & .0000 & $.0 \pi r a$ & .0000 & .0000 \\
\hline . & . & .unad & . & .0000 & conan \\
\hline .0000 & .0000 & .0000 & .0000 & .0000 & $.000 ?$ \\
\hline 1.0000 & .0000 & $.000 \Omega$ & .0000 & .0000 & .000 .0 \\
\hline .0000 & $1.000 n$ & .0000 & .0000 & .0000 & .0000 \\
\hline .0000 & - unan & - 0000 ? & .0000 & -.rano & .bann \\
\hline .0000 & .0000 & .0000 & .0000 & .0000 & $.000 \Omega$ \\
\hline $.000 \mathrm{C}$ & $.00 a$ & .0000 & .0000 & .0000 & .0002 \\
\hline . & $.000 n$ & . ח000 & .0000 & .0000 & 1. \\
\hline .000 & $\therefore .0000$ & ـLanan. & .0000 & acoco & .0000 \\
\hline
\end{tabular}




$$
\Delta x_{t-1 \quad \text { continued }}
$$

\begin{tabular}{|c|c|c|}
\hline .0000 & -.4849 & $Y_{r-1}$ \\
\hline .0000 & -.0908 & $M B_{r=1}$ \\
\hline . & -.0968 & $F R_{t-1}$ \\
\hline $.00 n$ & -.1817 & $C E_{t=1}$ \\
\hline .0000 & -.3470 & $Y D_{r-1}$ \\
\hline .0000 & -.4100 & If $_{+-1}$ \\
\hline .01000 & -.2309 & $C_{r-1}$ \\
\hline - onun & -.0722 & $I_{t=1}$ \\
\hline .0000 & .1817 & $1 M_{t-1}$. \\
\hline .0000 & $=L_{i} \varepsilon 2 \Omega$ & $M_{+\times r}$ \\
\hline .0000 & -.0003 & $R S_{t-1}$ \\
\hline .01000 & $=.0001$ & - $R L_{t=1}$ \\
\hline .0000 & .0850 & $C F_{t-1}$ \\
\hline -.0ana & $=.11631$ & $I_{r=1}$ \\
\hline - onor & -.01033 & $P_{t-1}$ \\
\hline . conar & .0000 & $E L I_{t-1}$ \\
\hline .0000 & $.000 n$ & $R L 2_{t-1}$ \\
\hline عمס0. & منعم. & $\mathrm{BL}_{3}{ }_{\mathrm{B}=\mathrm{T}}$ \\
\hline .0000 & - ciocon & $R S I_{t-1}$ \\
\hline .0000 & . & $R S Z_{t=1}$ \\
\hline .0000 & - ciouo & $P S Z_{t-1}$ \\
\hline $.000 a$ & مaمن. & $S M_{E=1}$ \\
\hline .0000 & $.000 \mathrm{C}$ & $I_{t-1}$ \\
\hline .0000 & .couan & $s x_{r=t}$ \\
\hline .0000 & .0000 & $S \times I_{r-1}$ \\
\hline مחمח.1. & . & $-S \times 2+-1$ \\
\hline
\end{tabular}




$$
\mathrm{Cu}_{t}+\mathrm{a}_{0}
$$

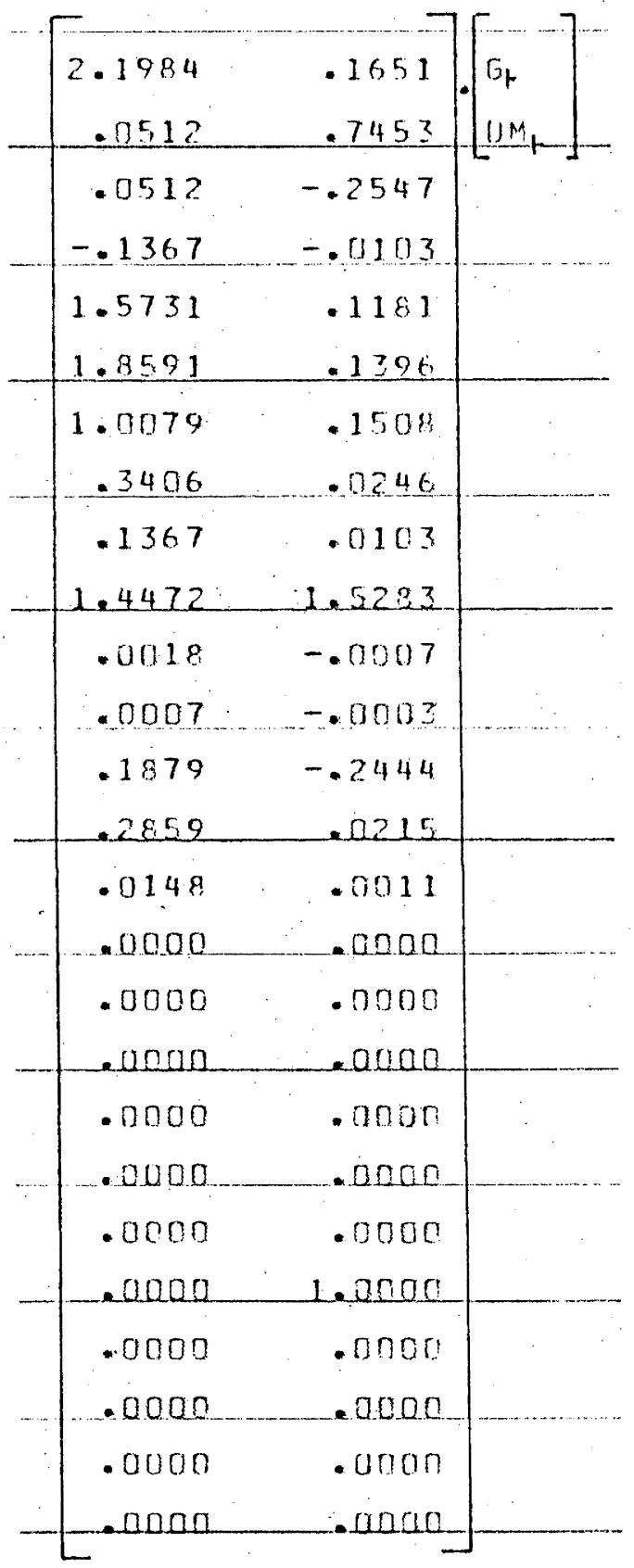

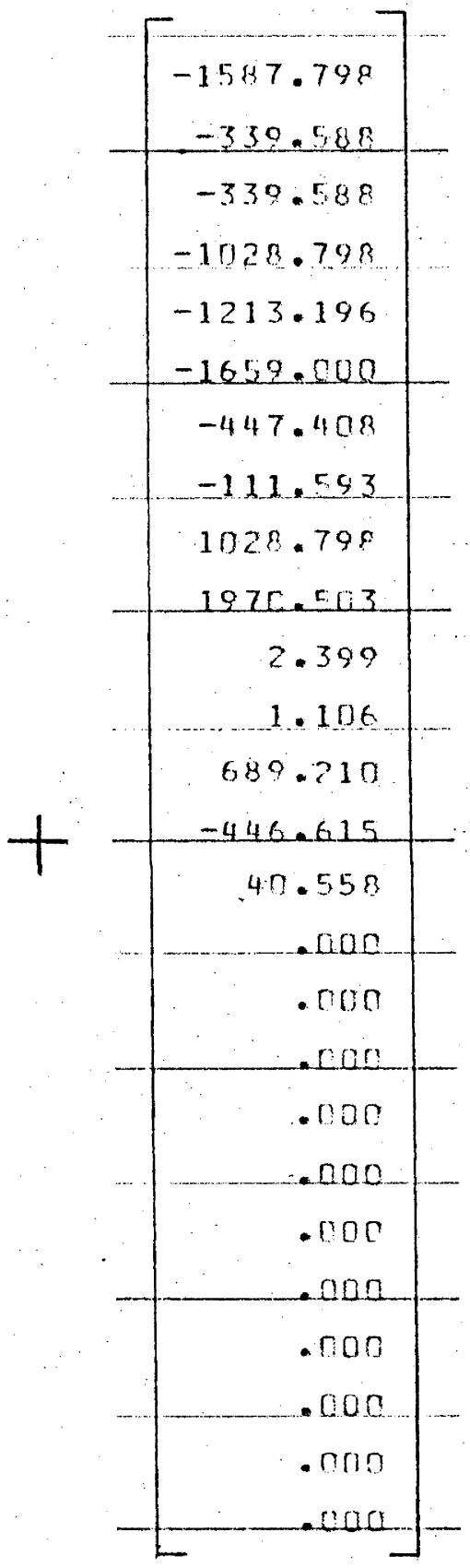


$\mathrm{Dz}$.

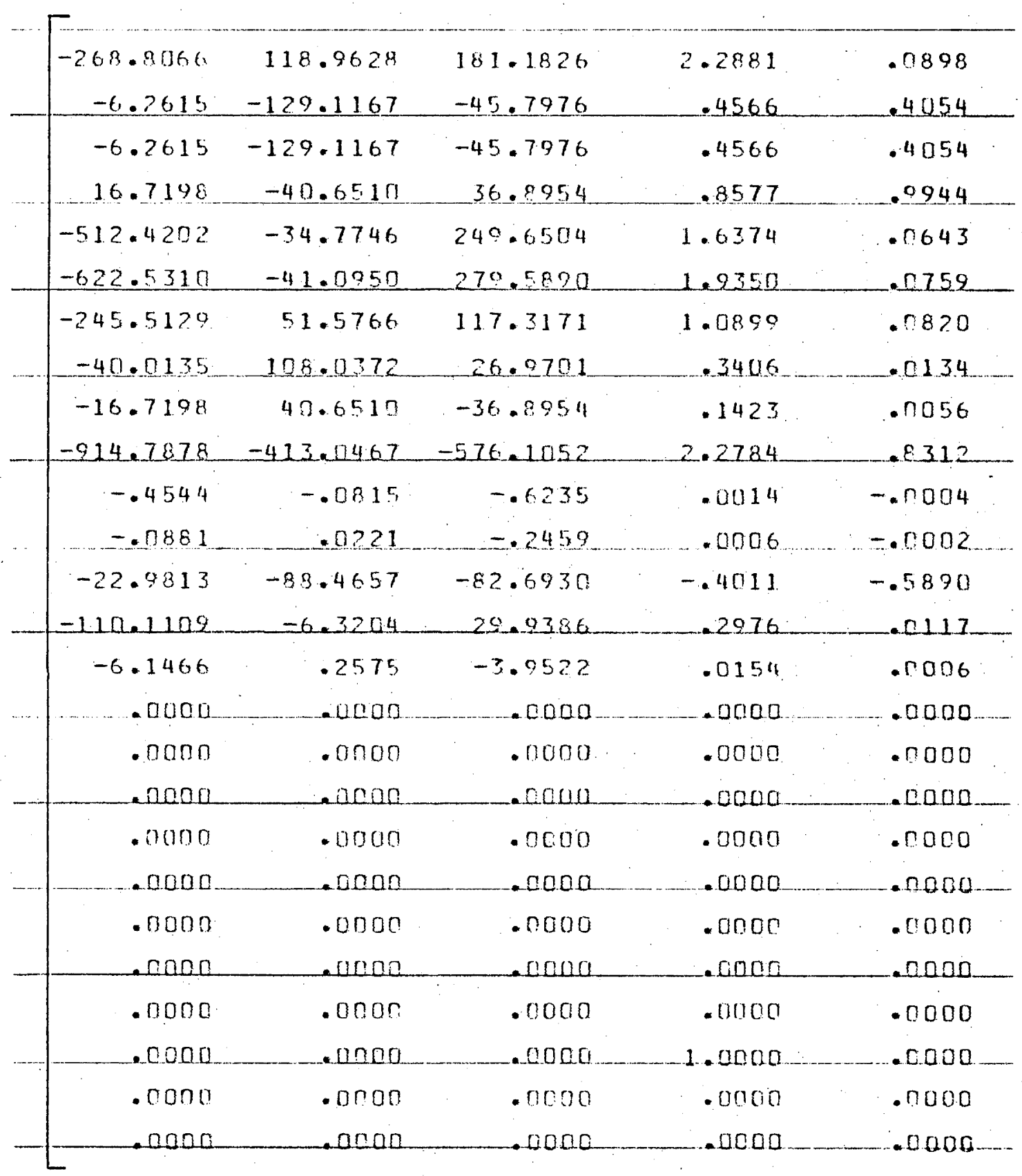


$\mathrm{D} z_{t} \quad$ continued.

\begin{tabular}{|c|c|c|c|}
\hline-1.6287 & 27.9547 & .0200 & -6.1208 \\
\hline$=.0372$ & 5.5782 & .0901 & -27.6327 \\
\hline-.0379 & 5.5782 & .0901 & $-27 \cdot 6327$ \\
\hline .1013 & 10.4784 & -0.0012 & .3807 \\
\hline$-2 \cdot 1655$ & 20.0043 & .0143 & -4.3800 \\
\hline-1.3773 & 23.6402 & .0169 & -5.1761 \\
\hline-1.3387 & 13.3151 & .0182 & -5.5904 \\
\hline-.3913 & 4.1612 & .0030 & -.0111 \\
\hline. .1013 & -10.4784 & .0012 & -.3807 \\
\hline-1.0721 & 27.8357 & -1847 & -56.6618 \\
\hline-.0013 & .0173 & -.0001 & .0265 \\
\hline-.0005 & .0068 & .0000 & .0104 \\
\hline-.1392 & -4.9002 & .0913 & -28.0134 \\
\hline$=.2112$ & 3.6359 & .0026 & -.7261 \\
\hline-.0110 & .3224 & .0001 & -.0413 \\
\hline $.00 u n$ & .0000 & .0000 & .000 \\
\hline .0000 & .0000 & .0000 & .0000 \\
\hline-.0000 & م0م0. & .0000 & مחمص. \\
\hline .0000 & .0000 & .0000 & .0000 \\
\hline .0200 & .0000 & .0000 & .0م0.0. \\
\hline .0000 & .0000 & .0000 & .0000 \\
\hline ـم00.0. & .0000 & مחمח. & مחمد. \\
\hline .0000 & .0000 & .0000 & .0000 \\
\hline-.0000 & .0.000 & .0000 & -anan \\
\hline .0000 & .0000 & .0000 & .0000 \\
\hline .0000 & .0000 & -.0000 & .0000 \\
\hline
\end{tabular}

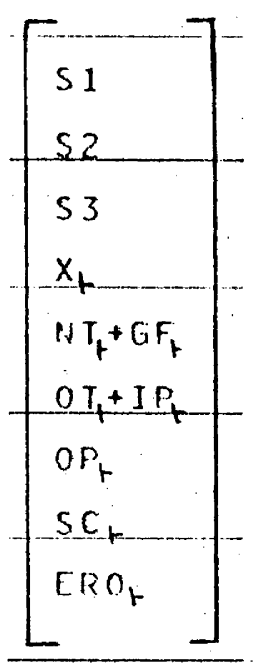


appropriate targets could be specified, there is no guarantee that the system could be moved into an upswing without generating unstable and unacceptable time paths for some endogenous and control variables. Even if the problem of the system structure can be overcome, the use of a mapping of the initial conditions into appropriate target vectors may produce unsuitable targets. The levels of income and other key variables may be so low at time $t_{0}$ (initial conditions at any particular point in time) that a mapping of these variables into targets could produce a set of targets which would not allow the system to move quickly enough out of the undesired situation. A similar argument holds for the case of an upswing, in particular the targets may be over-estimated in this case. For practical policy purposes a simple target rule would not be sufficient and a certain degree of intuitive assessment would be required to arrive at suitable targets. The discussion in Chapter two on some of the implications of uncertainty about the initial state vector suggests that policy planners would need to forecast the initial state vector in advance and if a simple target mapping procedure was employed it can be seen that if the forecast was incorrect then there will be some divergence between the estimated target vector and the "true" target vector which would be a function of the true but incorrectly forecast initial state vector. The breakdown between initial conditions and targets could make it more difficult to achieve desired targets. In the absence of any formal exhaustive theory of target specification, and it is beyond the scope of this study to produce one, the targets will be specified along the initial condition mapping lines outlined above although it is recognised that problems with this method do exist. It should be noted at this point that the very brief discussion of the topic outlined above merely touches on the whole problem of target specification. There is ample room for 
considerable research to be carried out in this area.

Before discussing the choice of target paths it is useful to look at the procedure of breaking up the endogenous variables into two categories, specifically, a subset of what could be termed target or ultimate objectives and a subset of intermediate objective. (For a succinct discussion of this classification see Norton (1973)). Ultimate objectives are usually classified as those which have some intrinsic value in their achievement, while intermediate objectives have no intrinsic value in their own right but are nevertheless important in helping to facilitate the achievement of the ultimate objectives. It should be clear that the distinction between ultimate and intermediate variables is very hazy and will largely rest on the particular philosophy or view of the economic system held by a particular individual. For example it is a simple matter to make variables either an ultimate or intermediate variable. A monetarist may focus on the supply of money as being the variable with the most intrinsic value while a "Keynesian" may regard the supply of money as being important only in the context of how it can facilitate the achievement of some other target such as income. This distinction will of course be important in the choice of appropriate stabilisation policies. An a priomi selection of ultimate and intermediate variables would severely limit the scope of applied analysis in either a flexible or fixed target analysis of policy as the ultimate targets would have desired target paths specified while the intermediate targets would not. To ensure that the following applied analysis can shift instantaneously between differing definitions of variables, target paths will be specified for all endogenous variables. Thus, in the linear/quadratic framework, by appropriate weighting in the cost function a variable can assume the role of ultimate or intermediate variable with 
a high positive weight for the former and a zero weight for the latter. This will enable the hypothetical policy planner to switch between income or supply of money as the ultimate target of the system, for example.

The specification of the target paths for the majority of the variables presented some difficulties due to the seasonality contained in the model and which results in time paths for the expenditure variables in particular, containing a substantial seasonal pattern. Experiments were performed, both optimal and non-optimal, in which the controls were used to try and remove the seasonality from variables such as $\mathrm{Y}, \mathrm{C}$, TPY and $\mathrm{T}$ and to keep them close to a trend line. This approach produced severe instability in the solutions, as could probably be expected, and was abandoned in favour of maintaining the seasonal variation. The technique of specifying targets and models in seasonally unadjusted terms has taken on more importance in Australia as a result of the government's decision in 1976 to abandon seasonally adjusted unemployment figures, largely for political purposes, in favour of seasonally unadjusted data. (Seasonally adjusted figures were subsequently reintroduced at a later date.) Model builders and researchers interested in the specification and estimation of Phillips curves, for example, may be forced to work in purely seasonally unadjusted terms and present forecasts in the same format. To complement this approach, the following technique was derived to establish a target path which is a derivative of the appropriate initial conditions and reflects the underlying seasonal variation of the variable concerned. Targets for the expenditure variables $C, I, I M, T P Y, T$ and the control variable $G$, were selected in the following way. Values for the first four quarters of the planning period were obtained by increasing 
the corresponding historical values of the previous four quarters by ten percent (remember that this is a ten percent increase in nominal terms only and refers to a twelve month projection from each initial quarter). The value of the December quarter was projected four quarters further ahead by ten percent, that is to the next December quarter twelve months later. A trend line was then drawn between the two projected values for the December quarter. Targets for all December quarters for the remainder of the planning period were taken to lie on the trend line. All other values, from the second year on, were obtained by maintaining the relationship between the December quarter and the remaining three quarters in the first year of the planning period with respect to the December quarter trend line. To enhance accuracy, future values of the targets were computed from linear relationships which represented the December trend line and which represented the appropriate linear trend of the remaining three quarters specified as above. The result was a set of targets that incorporated a uniform pattern of seasonality for each particular variable (not necessarily uniform between variables). As an example, the equations based on the above procedure which were used to derive the desired paths for $C$ and $G$ are given below.

$$
\begin{aligned}
& C_{1}=2875+91_{t} \\
& C_{2}=2975+91_{t} \\
& C_{3}=2950+91_{t}
\end{aligned}
$$

where the subscript on $C$ indicates which quarter the trend line refers to and the time variable, $t$, refers only to the successive quarters designated by the subscript. Similarly, the equations relevant to $G$ are given by 


$$
\begin{aligned}
& G_{1}=900+28_{t} \\
& G_{2}=1140+28_{t} \\
& G_{3}=890+28_{t} \\
& G_{4}=1010+28_{t}
\end{aligned}
$$

Equations for all the expenditure variables listed above were calculated in the fashion of those for $\mathrm{C}$ and $\mathrm{G}$. The chief advantage of the technique outlined above is that it avoids explosive values of the desired time paths towards the end of the planning period if a simple growth criterion is used. In particular, if we are trying to maintain the seasonal pattern of the data and merely let each quarter grow at a constant growth rate beginning with the corresponding quarter in the four quarter period immediately preceding the planning period, then a situation represented in Figure 6 will result where the gap between quarters increases greatly over time. Note that Figure 6 is overemphasised.

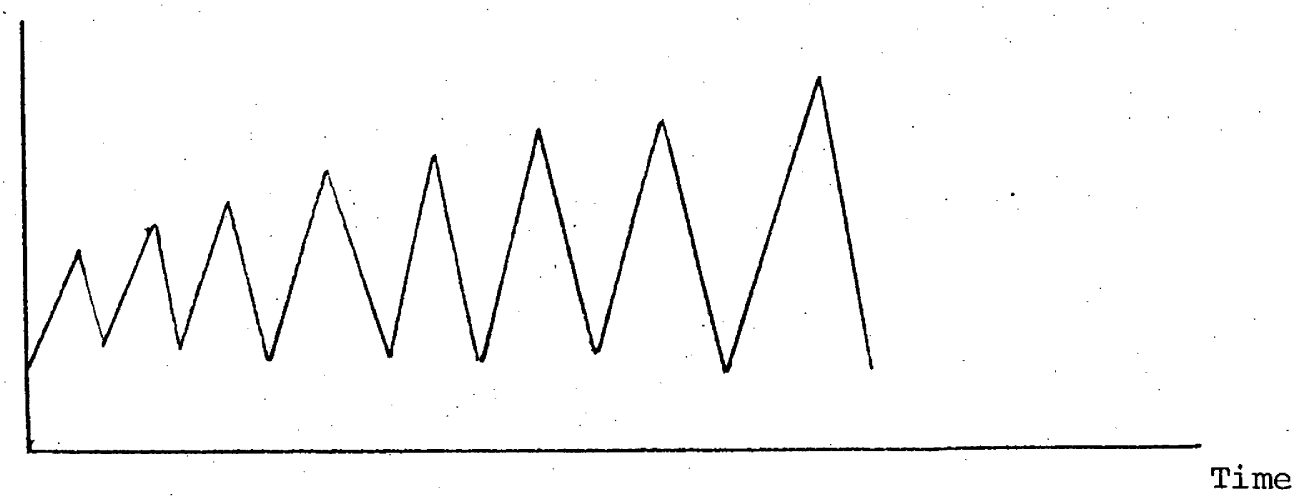

FIGURE 6

Explosive Seasonal Target Path

The target values of $C, I, I M$ and $G$ along with the historical values of the exogenous variable, $x$, were used to construct a target path for $Y$. Although the components of the $\mathrm{Y}$ identity add together to 
give a consistent target for $Y$ in terms of the components, there is no presumption that the resulting time path for $\mathrm{Y}$ would be able to generate the target level of the component variables. Therefore, the basic income identity contains possible inconsistencies. This assertion is best illustrated by a very simple example.

$$
\begin{aligned}
& Y=C+G \\
& C=20+.7 Y
\end{aligned}
$$

Let the target for $G, \bar{G}$, equal 100 and the target for $C, \bar{C}$, equal 200 . The target for $\mathrm{Y}$ is then found by adding $\overline{\mathrm{G}}$ and $\overline{\mathrm{C}}$ which gives 300 . On the surface this would appear to be consistent but if the target for $\mathrm{Y}$ is fed back into (4.21b) then the resulting $C \neq \bar{C}$. The position with the model developed in Chapter two is considerably more complex as feedback from I, IM and other variables is also important. The policy planner faced with this situation has two basic choices. The first is to set up the targets as have been done here and then compute $\bar{Y}$ and trade-off $Y$ and the component parts of the identity. Note that in some cases the component parts will not be treated as ultimate objectives and the problem will not arise. The second alternative would be to initially specify a desired time path for $Y$, and then allocate numerical values to the component parts such that in each time period they added together to give $\bar{Y}$. The major disadvantage of this procedure would be deciding the value of each component which in this procedure is entirely arbitrary. As well as that problem it should be clear that this procedure would suffer from similar inconsistency problems. This can be seen from the previous example by setting $\bar{Y}$ equal to 300 and letting $\bar{C}$ and $\bar{G}$ equal 200 and 100 respectively. The arbitrary nature of the second method suggests that the first may be preferable but it is not 
possible to arrive at any hard and fast rule as different target specifications will give varying degrees of trade-off. The above problem of consistently specifying targets within identities is very important in an applied framework and has, up until now, been largely ignored within the economic stabilisation literature with writers usually assuming that identities can be consistently achieved.

The targets for $\mathrm{RS}$ and $\mathrm{RL}$ were set at a constant $3.5 \%$ and $4.0 \%$ respectively which is slightly lower than the corresponding values contained within the initial conditions. The choice of targets for RS and $\mathrm{RL}$ is not as important as choosing targets for $\mathrm{Y}$ as in many experiments the rates of interest will be treated as intermediate variables and the time paths of desired values will only be used as bench-marks from which the dynamic behaviour of RS and RL can be studied. The target level of FR was kept at its historical value of the quarter immediately preceding the planning period (that is, its initiai condition). In other words, the balance of payments target was taken to be zero. The total money base and the money supply were specified time paths which grow at $1.5 \%$ per quarter and thus approximate one of the many versions of Friedman's (1968) rule. The target for the domestic component of the base was constructed by subtracting the FR target from the MB target. However, as with the case of $\mathrm{Y}$, there is no indication as to whether or not the targets of $\mathrm{FR}, \mathrm{MB}$ and $\mathrm{DM}$ can be consistently achieved. The time paths for all the exogenous variables were set at their historical levels thus the target for the current balance was found by combining the historical values of $\mathrm{X}$ and $\mathrm{GF}$ with the target values of IM. CF was then set equal to the positive value of the corresponding value of $\mathrm{CB}$ in each time period hence

$$
\mathrm{CF}_{\mathrm{t}}+\mathrm{CB}_{\mathrm{t}}=\Delta \mathrm{FR}=0
$$


Similarly, the target for YD was constructed from the specified paths of TPY, $T$ and the historical values of OT and IP. $P$ was the only behavioural equation to have a consistent target specified as its sole purpose is to act as a deflator for $Y$, and associated variables and provides a consistent link between the real and nominal sectors. The auxiliary variables defined to augment the state vector do not of course have target paths specified. As they constitute definitional relationships, they will always be equal to the lagged values of the variables they represent. The targets for all variables are set out in Table 2. Real targets are found by deflating by the price level. The targets derived above and set out in Table 2 constitute an acceptable and economically. meaningful (based on the initial conditions from which the majority were derived) target set with which to pursue flexible and fixed target stabilisation experiments.

\section{COST FUNCTION BIAS}

The erratic desired time paths of some variables suggest that in the linear/quadratic framework the use of one set of weights for the entire planning period may not be appropriate. The seasonal nature of other variables also suggests this as it can be seen that moving from seasonal peak to seasonal peak in say the target path for $Y$, would yield different quadratic penalties for equal percentage deviations from the target given a fixed set of preference weights. Previous applied optimal stabilisation studies, in addition to overlooking a wide variety of practical considerations, have overlooked the ramifications of employing one set of weights in the cost function for the entire planning period. In the linear/quadratic work of Chow (1972a), Pindyck (1973a) and Pindyck and Roberts (1974), one set of weights was employed for all time 
Table 2

Target Paths - Nominal Values

\begin{tabular}{c|c|r|r|r|r|r|r|r|r|}
\cline { 2 - 8 } Time & $\mathrm{Y}$ & $\mathrm{MB}$ & $\mathrm{FR}$ & $\mathrm{CB}$ & $\mathrm{YD}$ & $\mathrm{TPY}$ & $\mathrm{C}$ & $\mathrm{I}$ & $\mathrm{IM}$ \\
\cline { 2 - 9 } 1 & 5005 & 2619 & 1583 & -226 & 3365 & 3871 & 3066 & 884 & 898 \\
3 & 4760 & 2659 & 1583 & -264 & 3418 & 3942 & 3132 & 868 & 946 \\
4 & 5439 & 2698 & 1583 & -207 & 4036 & 4664 & 3548 & 962 & 951 \\
5 & 4930 & 2739 & 1583 & -304 & 3559 & 4085 & 3239 & 857 & 945 \\
6 & 5561 & 2780 & 1583 & -270 & 3795 & 4357 & 3430 & 981 & 951 \\
7 & 5341 & 2822 & 1583 & -339 & 3849 & 4428 & 3496 & 965 & 999 \\
8 & 6013 & 2864 & 1583 & -290 & 4464 & 5149 & 3912 & 1060 & 1005 \\
9 & 5568 & 2907 & 1583 & -176 & 3984 & 4571 & 3603 & 954 & 998 \\
10 & 6196 & 2951 & 1583 & -176 & 4215 & 4842 & 3794 & 1078 & 1004 \\
11 & 5899 & 2995 & 1583 & -301 & 4265 & 4914 & 3860 & 1063 & 1053 \\
12 & 6605 & 3040 & 1583 & -195 & 4852 & 5635 & 4276 & 1157 & 1058 \\
13 & 6060 & 3086 & 1583 & -296 & 4367 & 5056 & 3967 & 1051 & 1051 \\
14 & 6714 & 3132 & 1583 & -259 & 4596 & 5328 & 4158 & 1175 & 1058 \\
15 & 6460 & 3179 & 1583 & -307 & 4645 & 5399 & 4224 & 1160 & 1106 \\
16 & 7211 & 3226 & 1583 & -314 & 5258 & 6121 & 4640 & 1254 & 1111 \\
17 & 6690 & 3275 & 1583 & -220 & 4783 & 5542 & 4331 & 1148 & 1105 \\
18 & 7330 & 3324 & 1583 & -293 & 5005 & 5813 & 4522 & 1273 & 1111 \\
19 & 7222 & 3374 & 1583 & -263 & 5050 & 5885 & 4588 & 1257 & 1159 \\
20 & 7896 & 3424 & 1583 & -220 & 5674 & 6606 & 5004 & 1351 & 1165 \\
& 7464 & 3476 & 1583 & -159 & 5199 & 6028 & 4695 & 1246 & 1158 \\
\hline
\end{tabular}

All variables with the exception of $R S, R L$, and $P$ are measured in millions of nominal Australian dollars 
Table 2 (continued)

Target Paths - Nominal Values

\begin{tabular}{c|c|c|c|c|c|c|c|c|}
\hline Time & M & RS & RL & CF & T & P & G & DM \\
\cline { 2 - 8 } 1 & 11200 & 3.50 & 4.00 & 226 & 408 & 94.046 & 1168 & 1036 \\
3 & 11368 & 3.50 & 4.00 & 264 & 427 & 95.741 & 946 & 1076 \\
4 & 11538 & 3.50 & 4.00 & 207 & 525 & 96.457 & 1094 & 1115 \\
5 & 11711 & 3.50 & 4.00 & 304 & 429 & 97.669 & 1012 & 1156 \\
6 & 12064 & 3.50 & 4.00 & 270 & 463 & 98.070 & 1280 & 1197 \\
7 & 12244 & 3.50 & 4.00 & 290 & 580 & 100.174 & 1206 & 1281 \\
8 & 12427 & 3.50 & 4.00 & 176 & 483 & 101.571 & 1124 & 1324 \\
9 & 12613 & 3.50 & 4.00 & 176 & 517 & 102.181 & 1392 & 1368 \\
10 & 12802 & 3.50 & 4.00 & 301 & 536 & 103.120 & 1170 & 1412 \\
11 & 12994 & 3.50 & 4.00 & 195 & 634 & 103.980 & 1318 & 1457 \\
12 & 13188 & 3.50 & 4.00 & 296 & 538 & 104.651 & 1236 & 1502 \\
13 & 13385 & 3.50 & 4.00 & 259 & 571 & 104.799 & 1504 & 1549 \\
14 & 13585 & 3.50 & 4.00 & 307 & 590 & 106.425 & 1282 & 1596 \\
15 & 13788 & 3.50 & 4.00 & 314 & 689 & 107.704 & 1430 & 1643 \\
16 & 13994 & 3.50 & 4.00 & 220 & 592 & 109.463 & 1348 & 1692 \\
17 & 14203 & 3.50 & 4.00 & 293 & 626 & 109.523 & 1616 & 1741 \\
18 & 14416 & 3.50 & 4.00 & 263 & 644 & 111.804 & 1394 & 1791 \\
19 & 14632 & 3.50 & 4.00 & 220 & 743 & 112.751 & 1542 & 1841 \\
20 & 14851 & 3.50 & 4.00 & 159 & 647 & 115.108 & 1460 & 1893 \\
\hline
\end{tabular}


periods in a particular experiment. The above selection of authors is not meant to be exhaustive as almost the entire applied literature consists of such a procedure. The approach is not entirely confined to the linear/quadratic literature and examples can be found in Friedman's (1975a) piece-wise quadratic approach to stabilisation and numerous non-linear applications. As the study presented here is linear, the problems of a fixed set of weights will only be studied within that context. It is argued that the use of a fixed set of weights is not applicable in a situation in which variables are growing over time or vary considerably from time period to time period. The use of one set of weights for an entire planning period can lead to a bias in the penalties incurred in a cost function which in turn can result in an unwanted bias towards particular targets. As an example, consider two major objectives of the experiments that follow in this study, that is, the optimal achievement of internal and external balance. Internal balance is taken to be the achievement of the target for real $\mathrm{Y}$ (which is consistent with the target for $\mathrm{P}$ ) while external balance is a zero change in the level of foreign reserves. The target path for $Y$ increases considerably over time, especially in nominal terms which the optimal experiments are initially formulated in. In contrast, FR remains constant. If the policy planner desired a situation in which equal emphasis should be placed on both targets in the cost function, it would be inappropriate to assign one set of weights at the beginning of the planning period to be used for all time periods. In period one weights could be assigned which produced an equal penalty for one percent deviations in both targets. However, as the planning period progressed, the penalty associated with a one percent deviation of $\mathrm{Y}$. from its target would increase relative to that associated with FR due to the fact that 
the target for $\mathrm{Y}$ is increasing over time while $\mathrm{FR}$ is being pegged at a constant level. If the penalty associated with a deviation of one variable from its target is greater than the penalty associated with another variable or variables, then the system will react in such a way so as to keep the variable with the highest penalty more on target relative to other variables. The result would be that total costs over the planning period would be minimised as required. The example given above illustrates that there could be a tendency for the system to have a bias towards the $Y$ target at the expense of the FR target even though the initial intention was to have an equal weighting between the two. The bias would become more severe towards the end of the planning period. A similar situation could occur when policy planners decide to place a greater emphasis on one variable, or variables, relative to another. For instance, in the example given above the initial preference situation may have been one where a one percent deviation in FR from its target was required to have a quadratic penalty equal to five times the penalty of a one percent deviation in $\mathrm{Y}$. Assuming the cost matrix $Q$ was set up in a manner which reflected this preference pattern in the first period, it can be seen that as the end of the planning period approached, the gap between incurred penalties would close (this can be seen directly from the table of targets and using a simple numerical example). It is conceivable that under certain weighting patterns, one variable may be so large or become so large relative to another that any initial preference position is negated or perhaps even completely reversed. The consequence of this for applied stabilisation work is only too clear. By retaining one set of weights for the entire planning period of an optimisation experiment, we may create an unwanted bias which is strong enough to reverse the intended trade-offs. The presence of bias in a solution is 
not a foregone conclusion as it will depend crucially on the dynamics of the system and the impact of the instruments on particular targets, but it may be very difficult or impossible to ascertain the possibility of the existence of bias from merely examining the structure or reduced form along with the desired target paths of variables. The applied results obtained from a particular experiment may not indicate the presence of bias. How do we disentangle the influences of system dynamics, the effect of instruments and possible bias unless of course it is so severe as to be obvious? The answer is that in most cases and particularly for very large models, it will be very difficult to detect bias so the obvious solution would be to ensure that the possibility of bias is eliminated from the outset.

To overcome the problem of cost function bias the applied control theorist can adopt one of several approaches. Firstly, the concept of using only one set of weights for an entire planning period can be abandoned. Multi-period weighting matrices can be employed in place of a single weighting matrix, which will adjust through time to maintain the desired trade-offs and the correct relativity of penalties. In the example given above, the weight for FR could be maintained throughout, while the weight for $\mathrm{Y}$ could be reduced over time in order to compensate for the growth in $\mathrm{Y}$ and the seasonal variation contained within $y$. The use of varying weights in this fashion would strictly maintain the desired relationship between penalties. A second solution would be to formulate the entire system in terms of growth rates. This would entail keeping all targets constant over time. Under these conditions a single set of weights for the entire planning period would be acceptable. The use of a growth rate representation and estimation is not usually favoured by economists wishing to study short-run 
stabilisation so this option may not be acceptable in many cases. A further solution would be to use a single set of weights and set the weights so that penalties for percentage deviations in the last period are equal. This would solve the problem of end-point bias but could introduce bias in the early periods and would only be an acceptable solution if the final periods were considered to be the only periods of importance. The suggestion of varying the weights each time period appears to be the most acceptable solution and was adopted for the linear/quadratic experiments carried out for this study. Note that the problem of bias will not arise in the fixed target framework, where given the appropriate degree of anticipation and provided that the controllability requirements are fulfilled, the policy planner will exactly hit all his targets. The weights for the following experiments were constructed such that a one percent deviation from the target path of any variable included in the cost function of a particular experiment, would incur a penalty of 250 per time period. The choice of 250 is quite arbitrary with the choice governing the size of the weights and the scale of the subsequent welfare loss for a given experiment. There are no convenient units of measurement which can be used to measure the welfare costs. The values produced by a quadratic cost function can only be regarded as high or low relative to another cost. The weights for the first of the internal and external balance experiments (IEBI) discussed in Chapter 5 are given in Tables 3 and 4 and illustrate how the weights can be adjusted each time period to avoid cost function bias. The weights are constructed such that all weighted variables will incur equal penalties for equal percentage deviations. The cost matrix $R_{t}$ for that experiment was specified to be diagonal while only the first fifteen diagonal elements of $Q_{t}$ were considered in the assigning of weights. 
Table 3

Cost Matrix Weights - IEBl (chapter 5)

Diagonal Elements of $\mathrm{Q}_{t}$

\begin{tabular}{c|cccccccc|}
\cline { 2 - 8 } & $\mathrm{Y}$ & $\mathrm{MB}$ & $\mathrm{FR}$ & $\mathrm{YD}$ & $\mathrm{C}$ & $\mathrm{I}$ & $\mathrm{IM}$ & $\mathrm{CF}$ \\
\cline { 2 - 9 } 2 & .100 & .369 & 1.010 & .223 & .269 & 3.235 & 3.135 & 63.045 \\
3 & .112 & .358 & 1.010 & .216 & .258 & 3.353 & 2.823 & 28.174 \\
4 & .085 & .348 & 1.010 & .155 & .201 & 2.729 & 2.792 & 42.852 \\
5 & .104 & .337 & 1.010 & .200 & .241 & 3.443 & 2.831 & 52.599 \\
6 & .082 & .327 & 1.010 & .176 & .215 & 2.626 & 2.794 & 38.409 \\
7 & .089 & .318 & 1.010 & .171 & .207 & 2.712 & 2.530 & 25.022 \\
8 & .070 & .308 & 1.010 & .127 & .165 & 2.251 & 2.503 & 23.906 \\
9 & .082 & .299 & 1.010 & .159 & .195 & 2.778 & 2.537 & 44.424 \\
10 & .066 & .291 & 1.010 & .142 & .176 & 2.174 & 2.505 & 147.522 \\
11 & .073 & .282 & 1.010 & .139 & .170 & 2.239 & 2.280 & 27.870 \\
12 & .058 & .274 & 1.010 & .107 & .138 & 1.889 & 2.258 & 27.263 \\
13 & .069 & .266 & 1.010 & .133 & .161 & 2.288 & 2.286 & 42.860 \\
14 & .056 & .258 & 1.010 & .120 & .146 & 1.830 & 2.259 & 50.288 \\
15 & .061 & .250 & 1.010 & .117 & .142 & 1.880 & 2.066 & 21.552 \\
16 & .047 & .243 & 1.010 & .092 & .118 & 1.608 & 2.046 & 55.276 \\
17 & .057 & .236 & 1.010 & .111 & .135 & 1.917 & 2.071 & 27.146 \\
18 & .047 & .229 & 1.010 & .101 & .123 & 1.561 & 2.047 & 94.509 \\
19 & .049 & .222 & 1.010 & .099 & .120 & 1.600 & 1.880 & 48.273 \\
20 & .041 & .216 & 1.010 & .079 & .101 & 1.385 & 1.863 & 41.380 \\
.045 & .209 & 1.010 & .094 & .115 & 1.630 & 1.885 & 105.858 \\
\hline
\end{tabular}


Table 4

Cost Matrix Weights - IEBI (chapter 5).

Diagonal Elements of $R_{t}$

\begin{tabular}{c|cc|} 
Time & G & DM \\
\cline { 2 - 3 } 1 & 1.855 & 2.357 \\
2 & 2.827 & 2.185 \\
3 & 2.114 & 2.035 \\
4 & 2.470 & 1.893 \\
5 & 1.544 & 1.766 \\
6 & 2.260 & 1.648 \\
7 & 1.740 & 1.542 \\
8 & 2.003 & 1.443 \\
9 & 1.306 & 1.352 \\
10 & 1.848 & 1.269 \\
11 & 1.456 & 1.192 \\
12 & 1.656 & 1.121 \\
13 & 1.118 & 1.054 \\
14 & 1.539 & 0.993 \\
15 & 1.237 & 0.937 \\
16 & 1.392 & 0.884 \\
17 & 0.969 & 0.835 \\
18 & 1.302 & 0.789 \\
19 & 1.064 & 0.746 \\
20 & 1.187 & 0.706 \\
\hline
\end{tabular}


The remaining eleven state variables are auxiliary variables and do not need to be incorporated into the cost function. Each row of $Q_{t}$ or $R_{t}$ in Tables 3 and 4 lists the positive diagonal elements of $Q_{t}$ or $R_{t}$ for one period of a twenty period planning period. The use of time varying cost matrices to maintain exact preference relationships has another important consequence for applied stabilisation research. It is only under conditions of maintaining exact scaling of weights that valid comparisons of welfare can be made between different sets of target preferences and instrument preferences. The absence of exact scaling of weights for all experiments, that is not reducing weights to a common bench-mark, could mean that in many instances the comparison of welfare between two different choices of targets may be quite meaningless. This factor has also been completely ignored in the applied stabilisation literature.

\section{COMPUTATIONAL ASPECTS}

The program for computing otpimal linear/quadratic target and instrument trajectories was written by the author and compiled and executed on the Australian National University's Univac 1100/42. The basic aim behind the construction of the program was to produce an efficient, flexible and simple to use package which could be utilised by the non-specialist programmer or control theorist. The need for such a package in Australia as compared with say the United States where a multiplicity of programs exist, is quite obvious when it is realised that at the time of preparing this study only one other optimal package was available. The alternative package had been developed by the Australian Bureau of Statistics and Australian Treasury and was not and still is not, available to the general public. The Treasury and statistics package 
(Fitzgerald, Johnston and Bayes (1973)) is designed to handle non-linear piece-wise quadratic problems.

The linear/quadratic package developed for this study contains the following basic options.

1. The choice of constant cost matrices $Q$ and $R$ or time varying matrices to remove cost function bias.

2. A stochastic option which generates additive disturbances and simulates the system under stochastic control as well as deterministic control.

3. A test for instrument instability.

4. The computation of optimal controls under certainty and the resultant behaviour of the system when these controls are implemented in an uncertain (stochastic) world.

The choice of the above options is made through the use of simple control cards and once the basic data has been arranged it is a simple matter to adjust the control cards to utilise the alternative options. The program requires only $30 \mathrm{~K}$ of storage space thus making the application of optimal control to models of modest dimensions a feasible pursuit even with limited computing facilities.

\section{INSTRUMENT INSTABILITY AND SYSTEM DYNAMICS}

An analysis of fine tuning would not be complete without mention of the problem of instrument instability. Instrument instability first appeared in a formal framework in Holbrook's (1972) seminal paper in which he demonstrated that with the number of targets being equal to the number of instruments, instrument instability could occur during the exact stabilisation of the targets. The essential feature of Holbrook's 
analysis is that the instrument exhibits a dynamic structure, a point which is often ignored in discussions of instrument instability. The requirement that the instruments possess a dynamic structure is crucial as it can be readily shown and is also intuitively clear that without such dynamics, instrument instability of the type discussed by Holbrook cannot exist. The problem of instrument instability would appear to be of some interest to both theoretical and applied practitioners of macro economic stabilisation but has received comparatively little attention in the literature either before or after Holbrook's contribution. Cornwall (1965) analysed the problem within the context of simple fiscal models but did not provide an exhaustive theory while Chow (1973b) and Sims (1974) have attempted a more general approach to the problem but were unable to settle upon a final exhaustive framework for analysing the problem. Turnovsky (1974) has come closest to providing a general framework within the linear/quadratic context and provides the most general propositions. One major point to be gleaned from Turnovsky's analysis is that if all endogenous variables are to be treated as targets (that is they are all weighted in the cost function) and if a positive weight is attached to deviations of all instruments from the appropriate desired time paths then instrument instability resulting from instrument dynamics and similar to the type defined by Holbrook cannot exist. 15 If it is found that instability in the instruments does exist then the policy-maker simply attaches a weight to deviations of the instruments from the desired paths. The general conclusion obtained by Turnovsky and briefly outlined above appears to be completely opposed to the conclusion obtained by Chow whose work has been often quoted on this subject. The general conclusion obtained by Chow was as follows: 


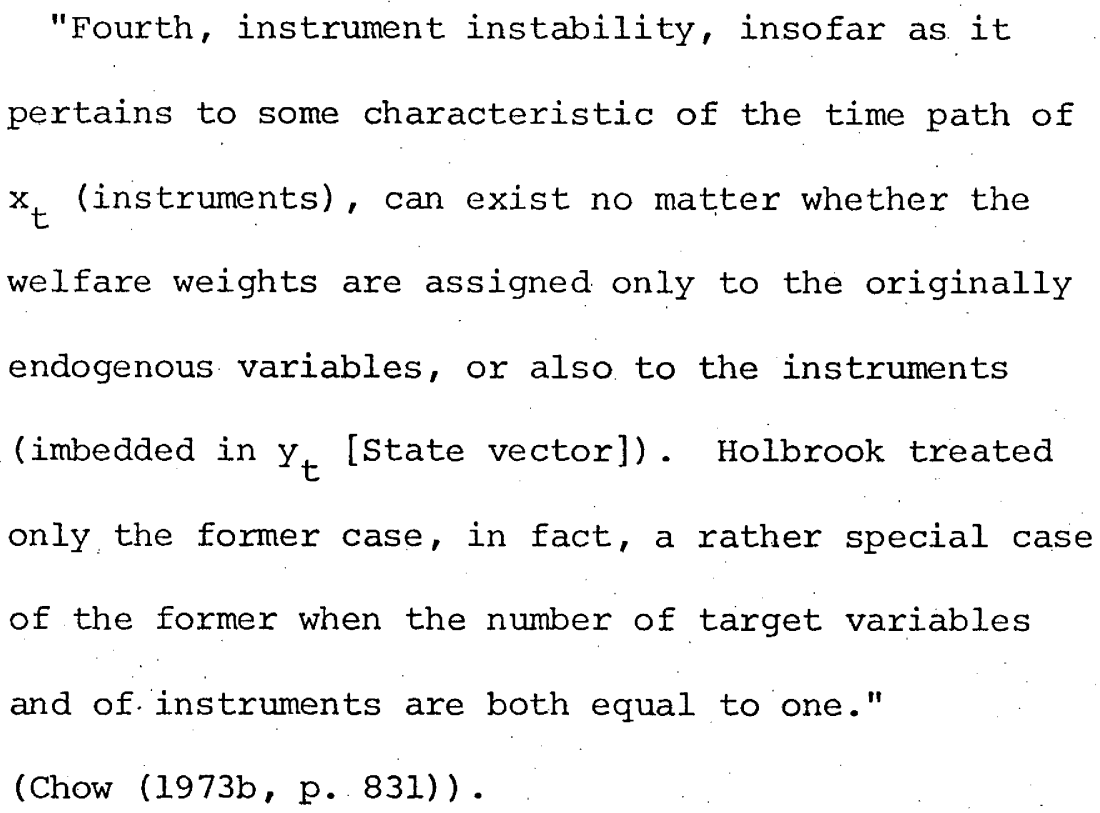

Can the conclusions of Turnovsky and Chow be reconciled? The answer is yes if it is assumed that in the Chow case only a subset of the endogenous variables are weighted in the cost function. If it is assumed that all endogenous variables are targets then using the Chow framework one must arrive at the same conclusion obtained by Turnovsky. However, if only a subset of endogenous variables are weighted in the cost function then it is possible for a type of instrument instability to exist even without any instrument dynamics. To intuitively illustrate this proposition consider the case where a policy planner wishes to stabilise two targets and has two instruments at his disposal and in addition there are other endogenous variables in the system which are not explicibly included in the welfare function but feed into the equations for the two targets. It is clear that if one or more of these intermediate variables are unstable then the impact of that variable on the targets could induce the instruments to behave in an unstable fashion as they will be forced to adjust to offset the unstable influence on the targets, which will in turn produce a further impact on the unstable variables thus requiring even further adjustment next period. 
The situation would be worsened if the instruments exhibited a dynamic structure which was also unstable. What is required in the above case is a redefinition of instrument instability to accommodate the type of instability outlined above and which is the only type of instability which can satisfy Chow's analysis when weights are assigned to the instruments, although chow does not seem to have recognised this. It is this omjssion which has caused the confusion which exists in some of the applied literature when it is asserted that instrument instability exists in the presence of costs on controls. The very short run reaction of the system to the initial conditions could convey the illusion of instrument instability as can the response of the instruments and the system as a whole to severe short run fluctuations in the uncontrollable exogenous variables. A panic reaction by the planning authorities to such short run fluctuations may result in heavy costs being assigned to particular instruments in anticipation of instrument instability when in fact such costs are not required. To avoid this type of mistake it would be desirable to ascertain a priomi to what degree, if any, instrument instability exists in the system to be controlled. The analysis of the existence of instrument instability could proceed in a number of ways. Firstly, if it was desired to control all endogenous variables and no instrument dynamics existed then instrument instability could be ruled out as it could if the problem satisfied the Turnovsky framework. (Turnovsky also briefly discusses the zero instrument cost case.) Secondly, if we were concerned only with a subset of endogenous variables, an indication of the existence of instrument instability could be gained by formally analysing the stability properties of the endogenous variables and instruments by computing the relevant characteristic roots (such a technique is 
advocated by Chow). Such a technique would be restrictive for very large models and an alternative procedure would be to apply the necessary conditions for discrete time stability of a system (see Baumol (1970). for discrete time and Gandolfo (1972) for an analogous set of continuous time conditions) which are considerably easier to compute than the characteristic roots. The necessary conditions are not sufficient, hence, if the system fails to satisfy the necessary conditions we can be sure that the system is unstable but we can never be sure of the stability of another system which passes the test for. necessity in the absence of a formal analysis of the characteristic roots. As a further alternative procedure, simulation studies can be carried out in which targets and exogenous variables are held constant over the duration of the planning period and the resulting behaviour of the controls is observed. As we shall see below, each technique, excluding the first mentioned examples where it can be seen quite clearly if instrument instability will be present, has problems at the application level.

A formal analysis of the characteristic roots of the system may tell the policy planner that instrument instability exists in the model either through pure instrument dynamics or through the presence of an unstable variable which is not included in the planners welfare function. Can it be inferred that instrument instability will be a severe problem under these circumstances? The answer is not necessarily yes as the degree of instability will be important as will the time span over which planning is to be carried out. Consider part (a) of Figure 7. The trajectories $u_{1}$ and $u_{2}$ (two alternative time paths for a given instrument) may represent instrument instability of differing degree. The government may be interested in stabilising the economy over the time 

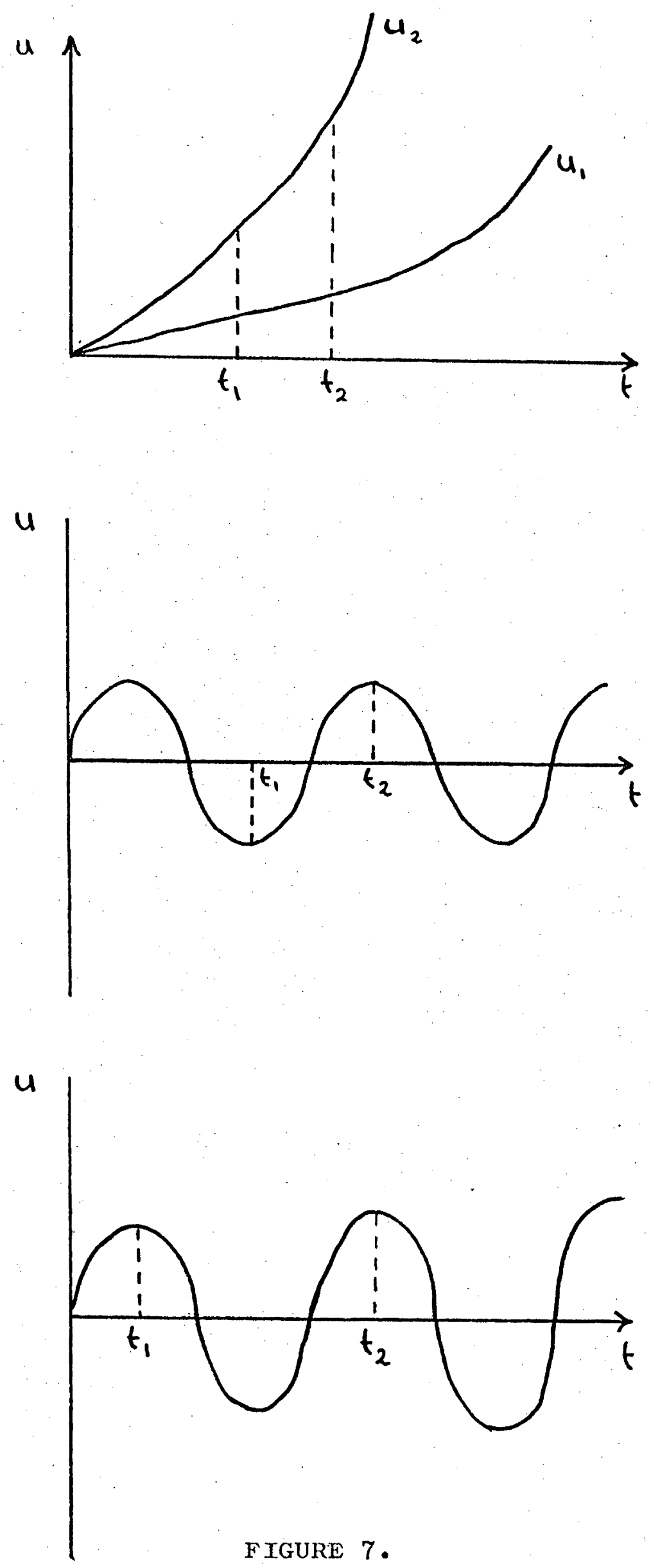

Degrees of Instrument Instability. 
span lying between $t_{1}$ and $t_{2}$. During that time span the trajectory $u_{1}$ exhibits little explosive behaviour even though it has been established that instrument instability exists. On the other hand, $u_{2}$ is more explosive relative to $u_{1}$ and may be of more concern. If instrument instability is of the type given by $u_{1}$ then the authorities may not take any immediate action as over the planning period, instrument instability would hardly be recognisable as such. The presence of a more explosive type could prompt the authorities to take action in the form of trading off targets against instruments to try and dampen the explosive nature of the instrument trajectory. Several writers including Chow, Turnovsky and Gramlich (1971) have queried this course of action arguing that if the prime concern of the authorities is the achievement of an ultimate objective then the unstable nature of the instruments will not be associated with any explicit costs and hence can be ignored. This view may be acceptable as long as the explosive behaviour of the instruments fails to generate any structural change in the system and the time paths of the instruments do not become politically unacceptable (regardless of how economically irrational this may be) or tax the resources of the government to a point where it becomes impossible to continue to implement the policy. The government may then be forced to abandon the fine tuning of the system in favour of a compromise course of action.

Part (b) of Figure 7 illustrates a situation in which the instrument exhibits constant amplitude oscillations, that is the relevant characteristic roots would be complex and the oscillations are the result of a sine or cosine function. The horizontal axis of part (b) does not necessarily refer to a zero position. In terms of policy, the axis can be referred to as a bench mark or some average level about which the 
values of the instrument oscillate. A formal analysis of the properties of the system which revealed non-explosive oscillatory behaviour of the instruments would indicate that instability is not a problem. For practical applications the time span of policy would once again be important, particularly if the time span $t_{1}$ to $t_{2}$ of part (b) Figure 7 was the relevant policy period. Given this situation it could be possible for non-government economic agents within the system to conclude that instrument instability was a problem resulting in the political and structural consequences outlined above, thus forcing the government to act as if instrument instability did exist. The absence of any formal analysis of the model and the use of a simulation approach could quite easily lead policy planners to conclude that instability exists when in fact the instrument is only on the upward phase of a constant oscillation. In the absence of a model and where historical observation is the only means of detection available, a similar conclusion could be arrived at. Similarly in the situation illustrated in part (a), a simulation study or historical observation may convey the wrong information, particularly if the underlying instability is of the type depicted by $u_{1}$. An historical analysis will not be adequate from the point of view that the targets may be increasing steeply over time thus requiring a stable instrument to increase steadily over time. This type of behaviour will not be instability of the type discussed by Holbrook, Chow, Turnovsky, Sims and Gramlich and could be corrected by revising downward the desired time paths of the target variables. Finally, the explosive oscillations depicted in part (c) of Figure 7 could be detected by formal analysis of the dynamics of the system but once again the degree of instability and the relevant time span is important. The explosive oscillations may only evolve slowly and will 
not be a problem for short run analysis, particularly if the planning period lies between $t_{1}$ and $t_{2}$ where the amplitude of the relevant oscillations is relatively close. The above observation has a general applicability to all forms of instability depicted in Figure 7 and that is that if instrument instability evolves very slowly then it can be largely ignored in the short run. It is highly unlikely that the same system structure will prevail too far into the future so that while mild instability may exist now, it may not exist in three or four years time. The problem here is that the government will not be able to accurately forecast the future structure of the system and may be forced to take unwarranted corrective action.

The problem of the correct structure is very important when examining instrument instability. If the intention of the optimal flexible target or fixed target experiments is to analyse historical policy and to suggest possible alternatives then it would be desirable to know if instrument instability existed over the particular period of interest. Historical observation of the instruments would not be completely effective for the reason given above and also due to the fact that the instruments may be reacting in the short run to severe fluctuations in the uncontrollable exogenous variables and the government may have been taking action and giving the impression that instrument stability (or for that matter target instability) did not exist. The only accurate means of detection would be a formal analysis of a model. If the model was incorrect structurally or specified correctly but estimated with data that produced wrong values for key coefficients then it would be quite easy to erroneously conclude either for or against instrument instability. The problem of the model structure becomes more severe when the model is used to plan for the future. Instrument 
instability may have been present over the estimation period and may have been captured accurately in the estimated parameters of the model but structural shifts in the future may remove the instability thus making the model inadequate for planning purposes. Most economists and econometricians would readily concede that models, regardless of their sophistication, are only simplified abstractions of reality and should only be viewed as such. The choice of abstraction however, could lead to the inclusion of instrument instability in a model when none exists in reality and vice versa. Under these conditions it is very difficult to make any definitive statements about stabilisation and control in the real world and as such we can only comment on stabilisation given a particular model. The results of any experiments with such a model should only be viewed as indicative in the sense that some insight can be gained into the stabilisation process but we are unable to make any concrete proposals.

Having looked briefly at the problem of instrument instability. and some of the difficulties in detecting it and detecting the severity of it, the model outlined in Chapter three will be briefly analysed. The first observation of importance is that government spending, or the fiscal instrument, only has a current period impact and thus if all endogenous variables are included in the cost function and zero costs are allocated to $G$ then $G$ will not exhibit instrument instability. The stability of the monetary instrument, DM, is not clear under conditions of including all endogenous variables in the cost function as DM possesses a dynamic structure through the lagged effect of DM on capital flows. The reduced form of the model set out at the beginning of this chapter gives the impact of $\mathrm{DM}_{t-1}$ on all endogenous variables and it can be seen that with the exception of $F R$ and $C F$, the absolute magnitude of 
the impact of $\mathrm{DM}_{t-1}$ is less than the absolute impact of $\mathrm{DM}_{t}$. This in itself does not prove the absence of stability with respect to those targets (exclusive of $F R$ and $C F$ ) but does indicate that it may be the case. $F R$ and $C F$ are subject to large impacts from $\mathrm{DM}_{\mathrm{t}-1}$ (relative to the impact of $\left(\mathrm{DM}_{t}\right.$ ) suggesting that a degree of instability could exist in the monetary instrument. A simple numerical test with the reduced form whereby targets for $F R$ and $C R$ are specified and then $D M$. is adjusted (G fixed) lends support to this assertion although it must be conceded that the feedback from other variables could have an influencing factor although this would not be expected to be very high due to the underlying dynamic structure. (See below.) Should instability in the monetary instrument be a problem? The answer is generally no as there is little reason for policy planners to attach any intrinsic value to its numerical value unless a monetarists type strategy is being adopted. The historical behaviour of DM illustrates that the monetary base (domestic) has been used in a very severe manner in response to adverse adjustments in the balance of payments but this may not be instrument instability in the strict sense as the monetary instrument settles down once the short run perturbation has been offset. The historical behaviour of DM serves to illustrate that the government attached very little, if any, cost to stabilising the monetary instrument as would be expected.

The experiments that follow only briefly focus on aiming for all endogenous targets within the system as in the case of the fixed target approach the use of only two instruments to stabilise so many variables would require a policy lead and policy interval of excessive and unrealistic proportions (provided that a policy sequence existed). In relation to the flexible target approach, the scant attention paid to 
stabilising all endogenous variables largely removes the analysis of instrument instability out of the general Turnovsky framework and places it within the context of only a subset of endogenous variables being weighted in the cost function with the possibility of unstable feedback behaviour of an uncontrolled variable causing excessive adjustment in one or both instruments (provided the costs on instruments are very small). The presence of an unstable endogenous variable does not guarantee instrument instability as the impact of such variables on the targets will also be important. If a variable is highly unstable and if it has a very small impact on a target then very little offsetting adjustment will be required by the instruments. Before proceeding to the internal and external balance experiments, each endogenous variable will be directly aimed for in the linear/quadratic framework with three alternative policy specifications. Firstly, both instruments will be aimed at the target and allowed to adjust in a free manner. Secondly, the fiscal instrument will be held constant while the monetary instrument is allowed to adjust freely and finally the monetary instrument will be held constant while $G$ is allowed to adjust. All exogenous variables will be held constant to avoid the problems of short run adjustment in the instruments in response to exogenous perturbations and the targets for the endogenous variables will be set equal to (for all time periods) the first period desired values set out in Table 2. The purpose of the experiments is to detect any feedback from the system into individual targets which is likely to produce instability in the instruments. The requirement that each instrument is held constant in turn will test the behaviour of the freely adjusting instrument in relation to a particular target. The case of both instruments free will indicate, if in fact it has been found that one instrument used by itself is unstable, whether 
the implementation of an additional instrument will dampen the use of the other instrument. It could be argued that what really is required is a formal dynamic analysis of the model but it has been argued above that the time span of the analysis is important. If the behaviour of the instruments is relatively stable over the desired period then it will not be of any concern that instrument instability is inherent in the structure of the model and takes a significantly long period to emerge. The analysis will only give insight into instrument behaviour with one particular target and it may be that the inclusion of an additional target will dampen instrument behaviour relative to the situation in which only one target is stabilised.. Nevertheless, the experiments will give some insight into the dynamic properties and instrument properties of the system and should prevent the mistake of attributing severely fluctuating instrument trajectories to "instrument instability" which is a common procedure in the stabilisation literature. A recent example of this can be found in the work of wells (1977) who attributes severely fluctuating instruments to instrument instability even though the structure of the model and his problem prevents the Holbrook type of instability from existing as is the case when it is analysed in terms of the more general linear/quadratic framework of Turnovsky. The only other possibility would be that the feedback from other variables is producing the instrument fluctuations but no attempt was made to disentangle this from the influence of exogenous perturbations and the reaction of the system to the initial conditions. The work of Wells is not the only study which contains such a loose approach to the term "instrument instability" as numerous examples can be found throughout the optimal stabilisation literature. 
regarded as a useful tool for gaining insight into the dynamic properties of stabilisation policies. It will be particularly useful. when the size of the model makes a formal dynamic analysis prohibitive. The format of the experiments will be as follows. (Note that all targets and exogenous variables are held constant.) Let $x_{i}$ equal the endogenous variable to be aimed for. The weighting patterns for the three experiments per endogenous variable are given by

$\begin{array}{cccc}\mathrm{x}_{\mathrm{i}} & \mathrm{G} & \mathrm{DM} & \\ 1000 & .1 & .1 & \text { Run 1 } \\ 1000 & 1000 & .1 & \text { Run 2 } \\ 1000 & .1 & 1000 & \text { Run 3 }\end{array}$

The allocation of low positive costs to $G$ and DM in Run 1 is essential as the stabilisation framework specifies more instruments than targets resulting in a failure of policy uniqueness and the non-existence of the inverse $\left(\mathrm{C}^{\prime} \mathrm{H}_{t} \mathrm{C}+\mathrm{R}_{t}\right)^{-1}$ when zero instrument costs are assigned. The costs are small enough to allow free adjustment of the instruments and ensure that a unique policy solution is obtained. For the case where both instruments are free to adjust, the results indicate that given the planning period length (twenty periods), the fiscal instrument, G, is very stable for most endogenous variables, allowing of course for a small adjustment at the beginning of the planning period due to the influence of the initial conditions. The reaction of $G$, when both instruments are permitted to adjust freely, when the target is the total base is one of damped behaviour. This is most likely due to the influence of $\mathrm{FR}_{t-1}$ on $\mathrm{MB}$ with $\mathrm{FR}$ being the most potentially unstable variable in the system due to the term $F R_{t-1}$ which appears in the identity for $F R_{t}$. The influence of $F R_{t-1}$ on the monetary variables generally produces a slight dampening effect on $G$ over the planning 
period. Of course it cannot be concluded that instrument instability does not exist but $G$ is at least stable over the twenty periods of the policy interval. The only target which produces cause for concern is $\mathrm{CF}$. The achievement of a constant $\mathrm{CF}$ target requires that $\mathrm{G}$ fluctuates between positive and negative values although the severe movements are not part of regular oscillary behaviour and would perhaps not be classified as instrument instability in the strict positive semidefinite cost matrix case. The behaviour of $G$ is due largely to the time path of $\mathrm{Y}$ which fluctuates severely and feeds back into $\mathrm{CF}$ via $\mathrm{CB}$ and $\left(\mathrm{Y}_{t}-\mathrm{Y}_{\mathrm{t}-1}\right)$. The movement of $\mathrm{G}$ to offset this influence produces further severe movements in $\mathrm{Y}$ which require further adjustment in $\mathrm{G}$. This result illustrates an important aspect of instrument stability in that the impact of a stable variable (stable in the strict dynamic sense) on a target can generate excessive movement in the instruments, particularly if there is a close trade-off involved as there is between $G$ and $Y .{ }^{16}$ Perhaps what is required is a redefinition of instrument instability to concompass the type of behaviour mentioned above and which may not fall into the category of "pure" instability which is essentially the type discussed by the abovementioned authors.

The behaviour of the monetary instrument when both instruments are allowed to adjust is similar to that of $G$, that is, stable but exhibiting slightly damped behaviour for $\mathrm{MB}$ and the other monetary variables. Like $G$, the only "unstable" behaviour occurs when $C F$ is treated as a target and occurs for similar reasons to those given above. $\mathrm{DM}$ must move to offset the influence of $\mathrm{Y}$ and $\mathrm{CB}$ and in doing so generates greater movements in these variables requiring more adjustment in later periods. Note that if $\mathrm{Y}, \mathrm{CB}$ and the other endogenous variables were weighted then the excessive movement in both instruments would be 
removed as the severe fluctuations in variables would be largely, although not completely, removed.

The remaining instrument experiments with $G$ and $D M$ alternatively fixed and free provide the most interesting and revealing insight into the behaviour of the system. The requirement that only one instrument can adjust in a free manner would suggest that the behaviour of the free instrument would be significantly more severe than corresponding behaviour of the fixed instrument. The general indicative results are given in Table 5. The heading "stable" refers to a time path which does not exhibit any tendency to explode upwards or downwards (columns three and four) or exhibits severe oscillatory type fluctuations which are covered by the second heading "severe movements". It can be seen that for the majority of endogenous variables, the monetary instrument is characterised by severe fluctuating behaviour or explosive type movements. Only the rates of interest are compatible with a relatively stable time path and even then some fluctuations do exist in the instrument time path but at a level far removed from the fluctuations contained in the time paths included in the second column. On the other hand the behaviour of the fiscal instrument presents a more uniform distribution between stable and significant downward movements. The stability of the fiscal instrument in relation to the income variables is not surprising and reflects the historical behaviour of $G$, if we assume that $G$ was historically aimed at income targets rather than open or monetary sector targets. The indications are that an incorrect assignment of fiscal policy could produce an unstable time path for the fiscal instrument while the assignment of monetary policy to a particular target without the assistance of fiscal policy, will most likely result in excessive fluctuations in the use of the monetary instrument. The 
Table 5

Instrument Behaviour - One Instrument Fixed

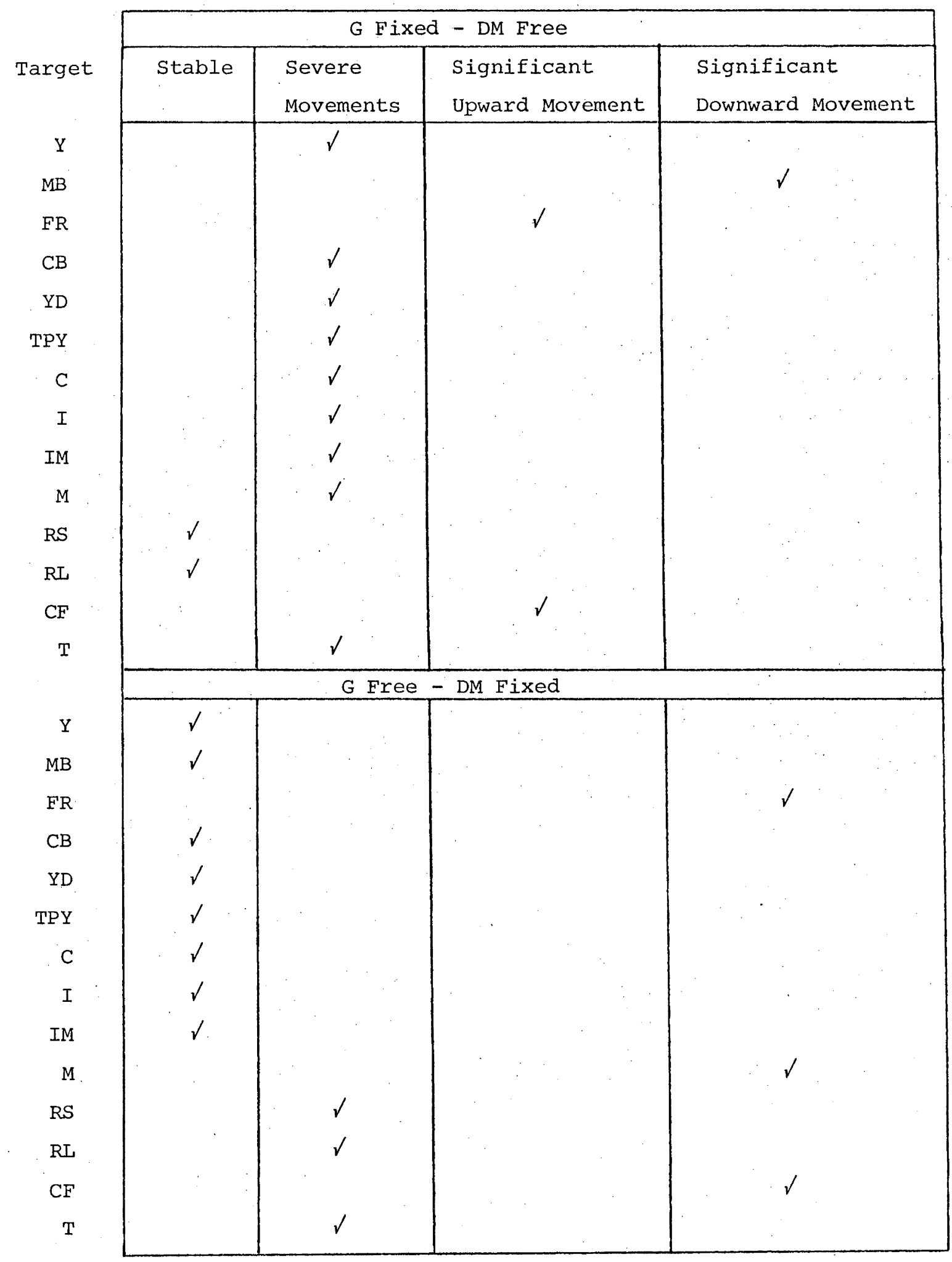

Note: Table headings refer to the behaviour of the freely adjusting instrument. 
important point to come out of the above analysis is that unstable instrument behaviour is possible within the constraints of the model and the analysis will assist in later experiments to ascertain whether or not severe fluctuations in the instruments, if there are any, are purely a function of any perturbations in the exogenous variables or are a result of the underlying structure of the model. It is likely that a combination of the two causes will be important but at least the preceding experiments will help to disentangle the problem. The results of the experiments also suggest that a combination of targets may remove instability, particularly if one target is compatible with a stable time path for one or both instruments. A further important aspect of stabilisation is suggested by the experiments and that is that it may not be possible to rely on one instrument or instruments of a similar nature to stabilise a given target or targets when the other instruments are specifically directed away from the desired targets. Further insight on this aspect will be gained from the following experiments.

The brief analysis of instrument instability carried out in this section not only gives insight into the particular dynamic instrument characteristics of the model but also presents a simple technique for detecting instability for particular targets when the time span is of concern and a formal analysis is prohibitive. A formal dynamic analysis would have been possible in this case but as it has already been pointed out even if instability had been present, if it did not become a problem until after the end of the policy interval then it would be of little concern. It may be of concern in the next policy interval but by then a structural shift may have occurred which would reverse the process. A more exhaustive approach to the problem would have been to test all combinations of targets with the exception of the case of including all 
endogenous variables in the cost function as the latter case would fall directly into the Turnovsky framework. Testing all combinations would be a computationally prohibitive process so only selected sets of targets were chosen. The limited results obtained largely confirmed the above assertions. An experiment was conducted within the Turnovsky framework with zero costs on the monetary instrument with the result that the time path for DM did exhibit a tendency to increase over time, although not in an excessively severe manner, suggesting that instrument instability of the more conventional analysis of stabilisation policy. It should be noted that the problem of instrument instability in a fixed target framework has been ignored mainly because at the time of writing no general theory had been developed in this context and was beyond the scope of this study to pursue such an investigation. ${ }^{17}$ Two observations can be made, however, concerning the fixed target approach. First of all it should be recognised that Holbrook's example is of a fixed target path problem (one instrument one target) and can be extended to include all strongly-Tinbergen dynamic stabilisation problems. Secondly, the situation in which the number of targets exceeds the number of instruments and a policy lead is required cannot be directly analysed in Holbrook's framework or the fixed target framework, but the results of the linear/quadratic experiments will give some indication of whether or not instrument instability may be a problem. The unsatisfactory nature of this approach can be counter-balanced by the fact that an alternative rigorous analysis within the fixed target, policy lead context does not yet exist. 


\section{CHAPTER V}

\section{OPTIMAL AND FIXED TARGET PLANNING FOR INTERNAL AND EXTERNAL BALANCE}

The analysis of this chapter focuses on the achievement of internal and external balance under a fixed exchange rate regime. Problems associated with uncertainty will also be considered. The linear/quadratic flexible target approach will be the dominant stabilisation procedure employed although the fixed target approach will be implemented for a particular set of targets, specifically in the case of the number of targets equalling the number of free instruments and the trading off of targets becomes unnecessary. Specific application of the dynamic fixed targets approach to a similar set of targets will be left until Chapter Seven.

The experiments that follow were carried out over a twenty period time horizon beginning with the second quarter 1965. In each case terminal conditions were not considered nor was the time horizon endogenous in the manner of Friedman (1975a) for any particular experiment. The choice of a twenty period time horizon was made firstly to enable a broad view of the optimal trajectories for both state and control variables to be obtained and secondly, to take account of the lag structure of the model. The longest lag in the model is a four quarter lag in the effect of the long rate of interest on investment. For the first four periods of the planning period only the initial values of $R L$ will be important while the values of the optimal RL over the last four periods will not be of any concern within the current planning period. In effect there would be four periods of any planning period (greater than or equal to four) in which the current level of RL would not have any impact within the time horizon and 
four periods in which the initial conditions and not the optimal values would be important. To try and gain some insight into how the system reacts in between these two subsets of the horizon it is necessary to sufficiently extend the horizon to obtain a period over which all lagged variables feeding back into the system are in fact optimal and the optimal values of the current lagged variables generated within the intermediate subset have time to work their way through the system. It is conceivable that given a model with very long lags (for example RBAl), the selected time horizon may be of a length which does not permit the optimal values of particular variables to feed back into the system and in a sense the model could not be regarded as evolving in a purely optimal manner. Strictly speaking, the solution will be an optimal solution but the absence of an optimal effect from certain variables will perhaps not yield sufficient information about the dynamic evolution of the system when all variables are optimal levels given a specific set of preferences.

The problem of long lags is important if the lags are as long as, or longer than, a specific time horizon and a new set of preferences (reflected in an allocation of new weights in the cost function) are assigned at the beginning of the next planning period when the optimal values of particular variables calculated over the previous time horizon are just beginning to have an effect. The result might be that the initial conditions may be so far away from the desired time path that the system takes an inordinately long time to settle down. A simple example of such a situation could be an initial set of preferences in which income and the balance of payments position axe the two targets of concern and the time horizon has been selected to be of the same length as the lag associated with the influence of the rate of interest on income. The achievement or near achievement of the two targets (depending on the number of 
instruments and how those instruments are weighted) may be consistent with a wildly fluctuating time path for the rate of interest which, at the end of the planning period, attains a very high value and has no impact within the current planning period. The next planning period may specify the supply of money as one of the targets which will depend on the complementary movement of the rate of interest but the rate of interest which influences the supply of money may be the rate which is consistent with the targets from the previous planning period due to the lags. involved, and may prevent the achievement of the new target. The practical consequences of this situation could be quite severe, particularly if there are many lagged variables in the system. The obvious approach to controlling such a system would be to avoid switching targets but this places considerable constraint upon the options of the policy planner. The problem of specifying the planning period to be of insufficient length and then switching state preferences will not arise in the experiments discussed below but the applied control theorist should be aware of some of the consequences of selecting an inappropriate time horizon.

The experiments were carried out within a deterministic and stochastic framework. The stochastic analysis was confined to considering the influence of random additive disturbances on the optimal trajectories of both state and control variables. The disturbances were generated by the computationally efficient procedure outlined by Nagar (1969) ${ }^{18}$. "In addition to a graphical presentation of the major results and comparisons of welfare cost between experiments and between stochastic and deterministic results for a given experiment, system performance will in some cases be summarised by the comparison of root mean square deviations (RMSD) of particular variables. The RMSD for state and control variables is given by 


$$
\begin{aligned}
\operatorname{RMSD}_{x} & =\left(\frac{1}{T} \sum_{t=1}^{T}\left[x_{t}^{*}-\bar{x}_{t}\right]^{2}\right)^{\frac{1}{2}} \\
\operatorname{RMSD}_{u} & =\left(\frac{1}{T} \sum_{t=1}^{T}\left[u_{t}^{*}-\bar{u}_{t}\right]^{2}\right)^{\frac{1}{2}}
\end{aligned}
$$

where the $x^{*}, \bar{x}, u^{*}$ and $\bar{u}$ refer to optimal and desired values of the state vector and optimal and desired values of the control vector. The RMSD purports to illustrate the overall performance of a variable with respect to its desired time path. The results however, must be viewed with caution as one result for given time period can influence the final value of the RMSD for a specific variable. To avoid drawing any incorrect conclusions from the value of the RMSD, the actual values of the variables were inspected and if behaviour of the type mentioned above was detected then an appropriate comment was made below during the discussion of the results.

The experiments for internal and external balance are identified by the notation IEB followed by a figure signifying the number of the experiment. IEBI consisted of attempting to achieve internal and external balance along with a number of other targets. That is, exact fine tuning was sacrificed in favour of a compromise approach. On the expenditure side $\mathrm{Y}, \mathrm{C}, \mathrm{I}, \mathrm{YD}$ and IM were all assigned weights which would provide equal penalties for percentage deviations from their respective targets (see Table 3 of Chapter 4). In the monetary and external sectors, $M B, F R$ and $C F$ were assigned weights which would produce penalties of the same magnitude as those incurred by the expenditure variables. $P$ was excluded from the cost function as the target was specified consistently with the target for $Y$ and therefore the inclusion of $\mathrm{P}$ would bias the solution towards the target. Both control variables were weighted in the cost function on an equal basis 
with the endogenous variables. M, RS and RL were treated as intermediate variables in the sense outlined in the previous chapter (not to be confused with the notion of intermediate targets used by Brunner (1969) and other monetary economists) and were not allocated weights. Similarly, the remaining variables not included in the cost function were regarded as intermediate variables. The relative weighting in the cost function for IEBI was

$\begin{array}{cccccccc}\text { Y } & \text { MB } & \text { FR } & \text { CB } & \text { YD } & \text { TPY } & \text { C } & \text { I } \\ 1 & 1 & 1 & 0 & 1 & 0 & 1 & 1 \\ \text { IM } & \text { M } & \text { RS } & \text { RL } & \text { CF } & \text { T } & \text { P } & \\ 1 & 0 & 0 & 0 & 1 & 0 & 0 & \\ \text { G } & \text { DM } & & & & & & \\ \text { I } & 1 & & & & & \end{array}$

Multiple cost matrices were used to maintain the exact relativity and preferences. The decision as to whether or not to include a particular variable in the list of targets or to treat it as an intermediate variable, depends largely on the political philosophy of the planner involved, as does the proportionate weighting of various targets which are to be included in the cost function. The purpose of the experiment is largely exploratory and to examine the underlying trade-offs between variables and to test the degree of adjustment in the controls when the controls are weighted equally with the endogenous variables. Unless otherwise specified the discussion of the results will refer to the deterministic solutions.

As was to be expected in the case of multiple targets, the system failed to achieve all targets simultaneously due to the trade-offs between variables. The results for key variables are presented graphically 
in Figures 8 to 11. Real and nominal $G$ begins close to its target and by the end of the planning period moves significantly above the target level. However, this strong movement in $G$ is not sufficient to maintain real $\mathrm{Y}$ at or above its target. Real $\mathrm{Y}$ is virtually on target at the beginning of the planning period but begins to move below its target as the end-point of the planning period approaches. This behaviour is not confined solely to $\mathrm{Y}$. A similar pattern emerges in real $C$, the component of greatest magnitude in the $\mathrm{Y}$ identity. In contrast to $\mathrm{Y}$ and $\mathrm{C}, \mathrm{I}$ performs better. Its time path for both real and nominal values is considerably above the desired target (although in terms of the quadratic cost function this will also be penalised). Possibly I would have exceeded its target by a greater amount if the long rate of interest had been closer to, or even below, its target. Higher levels of $M$ would be required to achieve this which would require a more expansionary monetary policy than was provided by the optimal solution. The exclusion of $C$ and I from the cost function would have allowed these variables to move in a manner complementary to the income target. The costs imposed on them prevents this action.

The other target of special concern in relation to this set of experiments, FR, behaves in a manner similar to $\mathrm{Y}$ in that it moves in a downturn towards the end of the planning period. This behaviour is the result of two main influences. Firstly, capital flows experiences severe one-period deviations from its target which are not counter-balanced by an opposite movement in the current account balance. These sudden deviations are sufficient to shift $\mathrm{FR}$ from its target and keep it there. The nature of the FR. identity ensures that this will occur. (3.24) and (3.25) illustrate that any shift away from the desired level of FR can result in a permanent departure from the target, even if $\mathrm{CF}$ and $\mathrm{CB}$ 


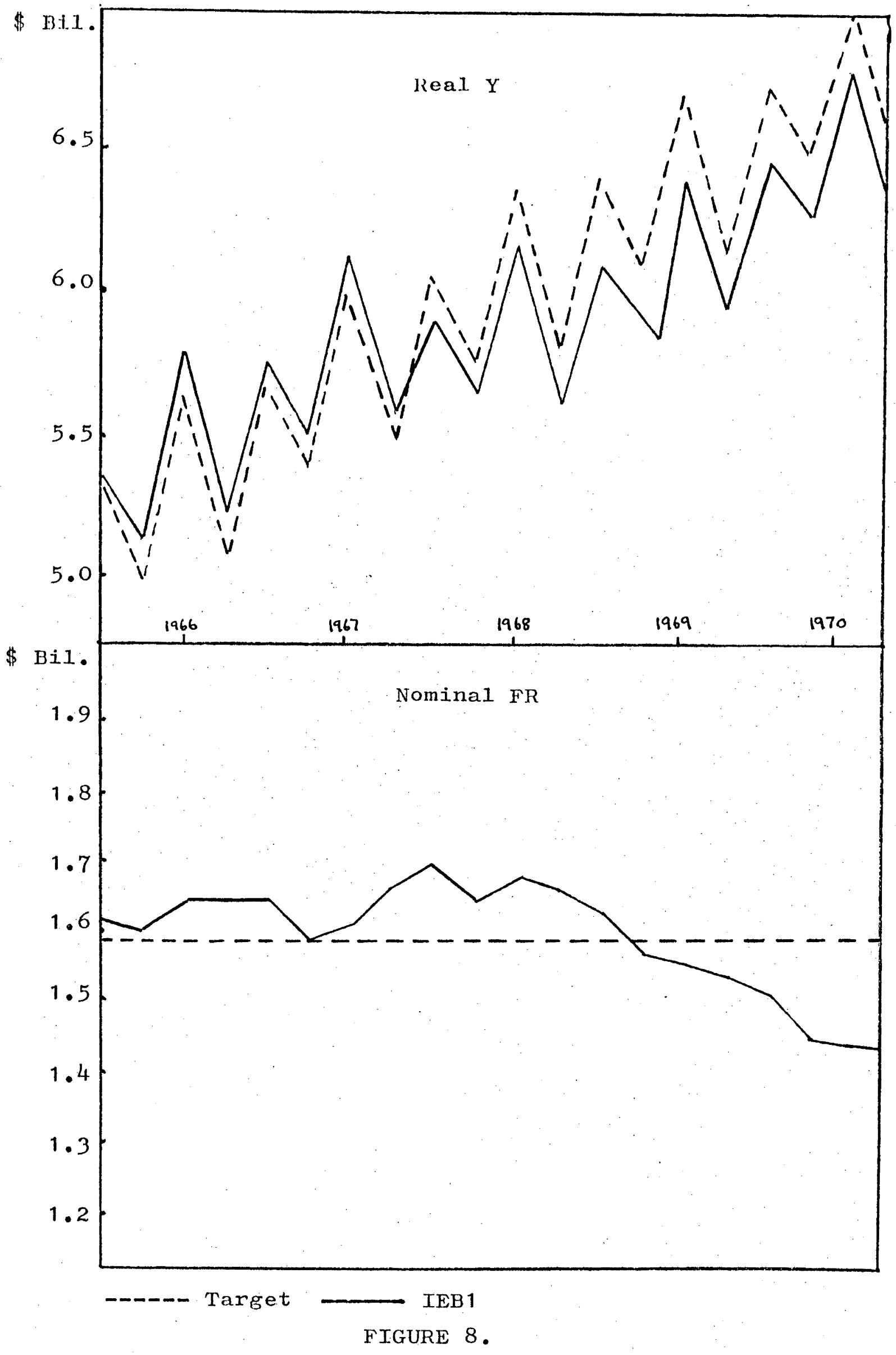




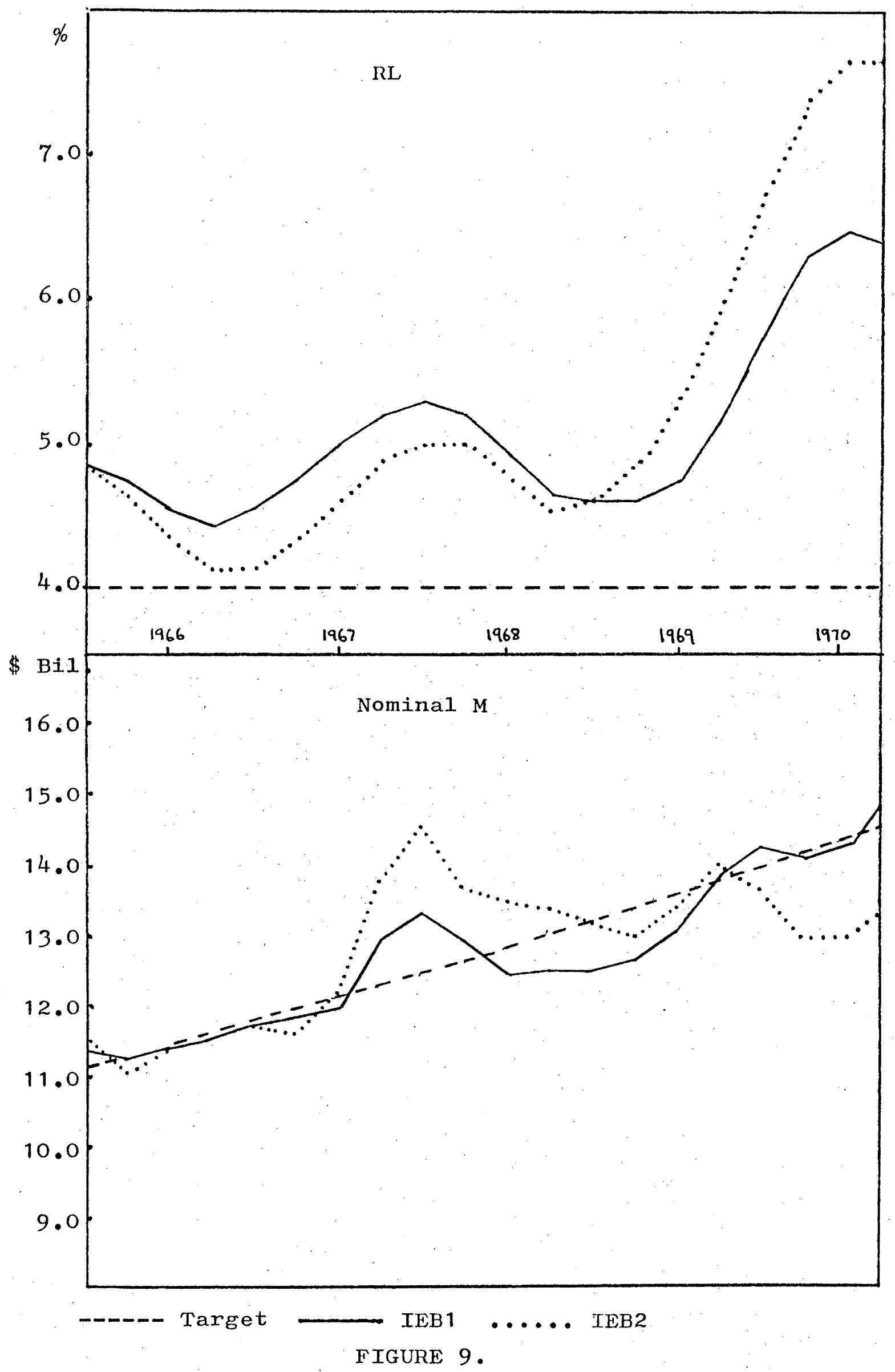




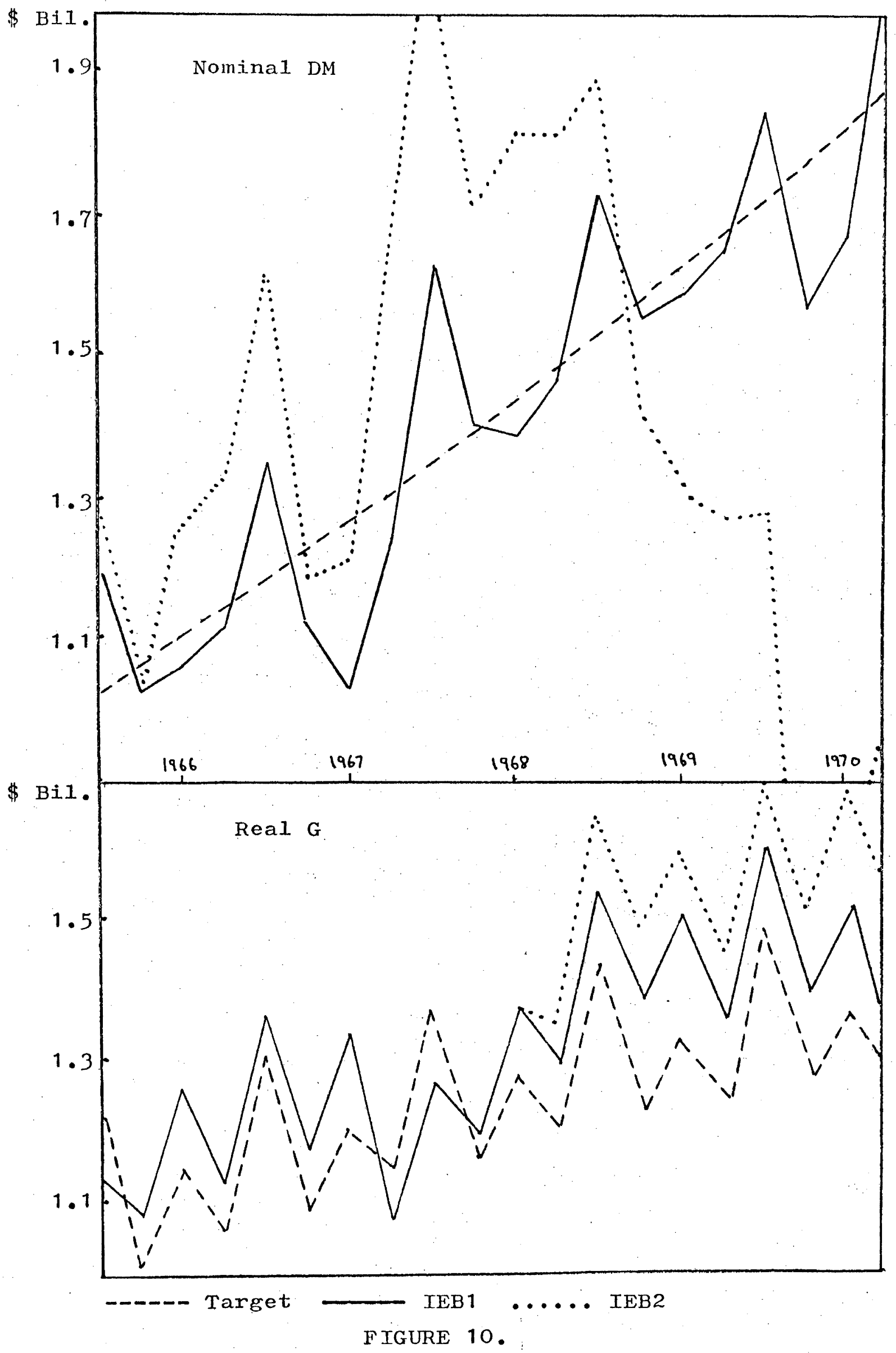




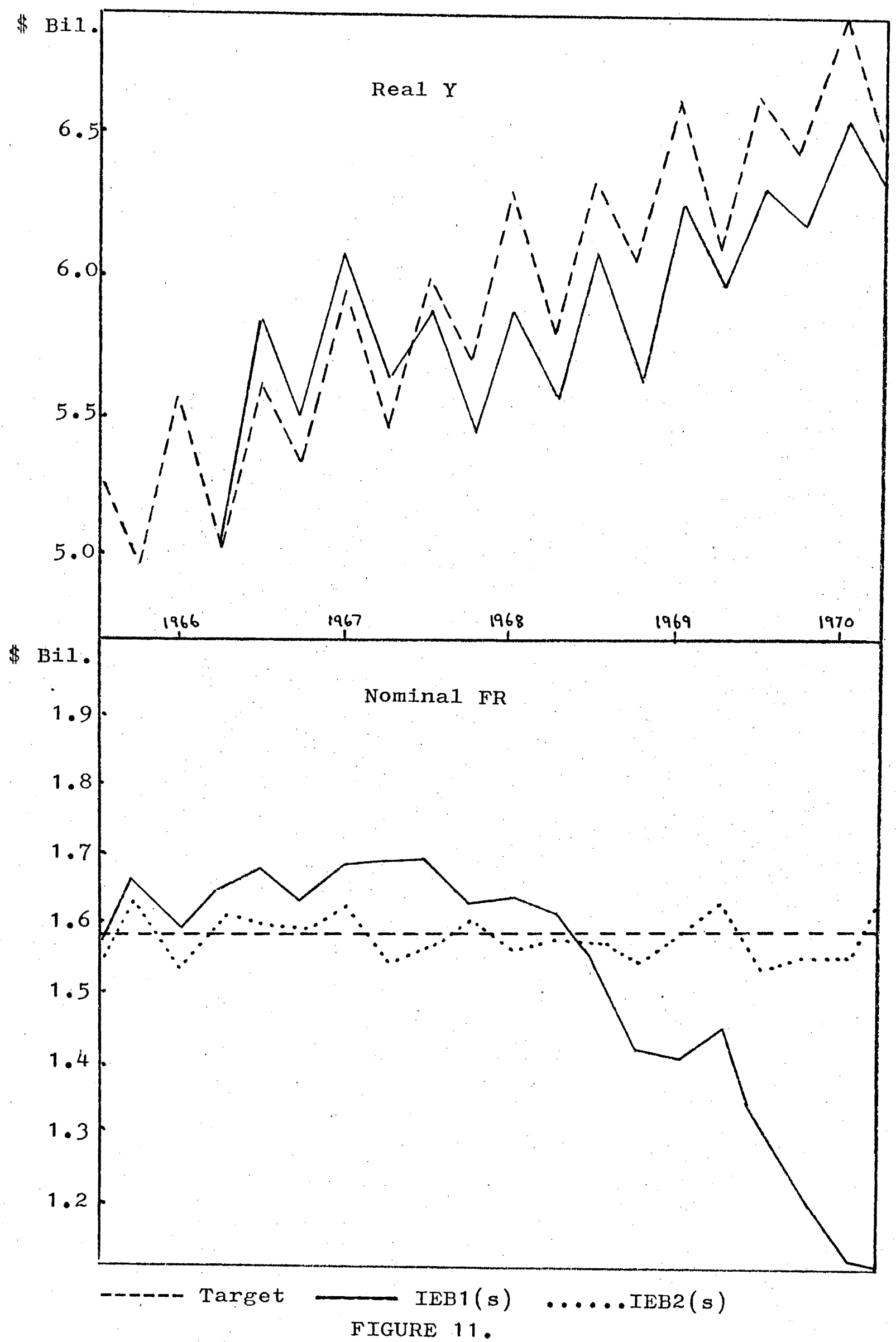


return exactly to their target levels. That is, the change in FR can be on target but if it is added to a lagged value of $F R$ that is off target, then the level of FR will be off target. Secondly, any movement off target by $\mathrm{FR}$ which results from $\mathrm{CF}$ movements, would require strong monetary action to return FR to its desired level. This can be seen from the structural and reduced form equations. The optimal solution does not provide for this monetary policy action due to the multiplicity of monetary and external targets which are weighted equally with FR.

The behaviour of the monetary instrument, DM, is not governed by the behaviour of $\mathrm{CF}$ and $\mathrm{FR}$ alone. It also has a major impact on the total monetary base $M B$ and a lesser impact on $Y$. The reduced form coefficients reveal that $\mathrm{DM}$ moves $\mathrm{MB}$ and $\mathrm{CF}$ in an opposite direction to that of FR. The structure of the experiment required that the system achieve targets for $\mathrm{MB}, \mathrm{FR}$ and $\mathrm{CF}$ simultaneously as well as allowing for a smooth domestic monetary expansion. The optimal results clearly show that these objectives are inconsistent and as a result monetary policy is used in an occasional stop-go fashion to try and produce the best trade-off in terms of the lowest penalties. In comparison to the optimal fiscal policy which allows for a steady but strong ${ }^{19}$ fiscal expansion, the optimal monetary policy is more volatile and more prone to be used to offset adverse external and monetary movements in the very short run.

The behaviour of the intermediate monetary variables $M, R S$ and $R L$ also requires some attention. Because these variables were excluded from the cost function, they were permitted to adjust fxeely in accordance with the requirements of the rest of the system. In this respect they are complementary to the control variables. $M$ is virtually on target both at the beginning and end of the planning period but like DM, it is subject 
to short expansion and contractions which occur in the middle portion of the planning period and which are largely a direct result of the behaviour of the monetary instrument. The fluctuations in the money supply contribute substantially to the "wave-like" time paths of the interest rates. Interest rates lie above their respective targets for the entire planning period and towards the end of the planning period they begin to rise steeply. This movement of interest rates and the money supply illustrates a very important problem associated with the use of intermediate variables in an optimal framework. The need to steer the system towards particular targets may create some instability in intermediate variables which could produce problems in the next planning period. For example, due to the steep increase in the long rate, some attempt may be needed in the next period to bring $\mathrm{RL}$ down in order to maintain stability within financial markets and to remove the detrimental effects of high interest rates on investment and income. This may mean the specification of $\mathrm{RL}$ as an explicit target which could produce further instability in $M$ due to the close simultaneous relationship between $M$ and the rates of interest. (See structural equations.).

The stochastic solutions to IEBI were initially obtained by a monte carlo technique whereby the solution was obtained fifty times, each with a new set of random additive disturbances. As could be expected the time paths of the endogenous variables displayed a tendency to converge towards the deterministic results. The monte carlo experiments when viewed as a whole failed to contribute much additional information to the deterministic results. A close inspection of the individual time paths of the state and control variables does provide some interesting information, particularly in relation to the response of the controls to the additive disturbances. As it would be impossible to present the results of each 
monte carlo replication, one replication was chosen to be used for illustrative purposes and the additive disturbances generated for that example were used in each illustrative example that follows to enable feasible comparisons to be made. The use of one set of disturbances to illustrate certain propositions has been used elsewhere in the stabilisation literature, for example, Pindyck and Roberts (1974).

The stochastic solutions do not provide any significant change in the behaviour of the weighted state variables if allowances are made for the introduction of the additive disturbances. In fact, the upper and lower time paths for real $Y$ resulting from the monte carlo simultations are very close to the desired path. The illustrative stochastic paths for $Y$ and $F R$ are given in Figure 11. The welfare loss generated under stochastic conditions increased by approximately 100\% with the majority of this welfare loss coming from the behaviour of FR and the controls. It can be seen from Figure 11 that additive shocks can severely influence the time path of $F R$ which is a direct result of the nature of the FR identity while the behaviour of $\mathrm{Y}$ varies little from the deterministic case. On the monetary side the movement of $M$ becomes more severe as does the movement in $\mathrm{RS}$ and $\mathrm{RL}$ with the income sector being largely insulated from the shocks to $M$ by the low coefficient for $M$ in the consumption function. The additive disturbances can move variables closer to targets or further away from targets depending on the size and direction of shocks. In light of this and the fact that $Y$, for example, substantially follows its deterministic path in the illustrative case (as it does in many others) and $G$ is weighted equally with $Y$, it would be reasonable to expect that $G$ would not vary substantially from the deterministic solution. This is not the case. In the deterministic solution the optimal fiscal policy required that $G$ exceed its target values. In the 
stochastic example, $G$ tends to decline relative to its target over the last few periods of the planning period. By comparison with the deterministic case, this represents the beginning of a more vigorous and contractionary fiscal policy. Recall that DM was used more extensively than $G$ in the deterministic case. Monetary policy is still used extensively in the stochastic case but in addition we see that $G$ is manipulated in a more extensive manner. The RMSD for $G$ increased by a factor of 4 from the deterministic solution compared to an increase of 2.5 for DM.

The stochastic case produces a new optimal mix of monetary and fiscal policy which produces the same general system behaviour as the policy mix in the deterministic case. However, the change in the behaviour of $G$ raises the question of whether or not the presence of uncertainty in a system can in fact reverse the application of a particular instrument to achieve an optimal trade-off between targets. The following three experiments provide some important information concerning this question. Before proceeding to the next experiment it is of some interest to briefly compare the historical behaviour of the economy with the simulated behaviour. It could be argued that the weighting specification used for the above experiment would not be followed as a practical stabilisation exercise as the controls are weighted equally with the state variables and while there may be some argument for weighting fiscal policy in this manner to take account of social needs for example, there is little justification for attaching the same importance to the monetary instrument. The interesting thing is, that despite the apparent restriction on DM, the monetary instrument has been used more extensively in the deterministic case than the corresponding historical policy and FR has been stabilised slightly more effectively than the historical time path for FR. (Figure 


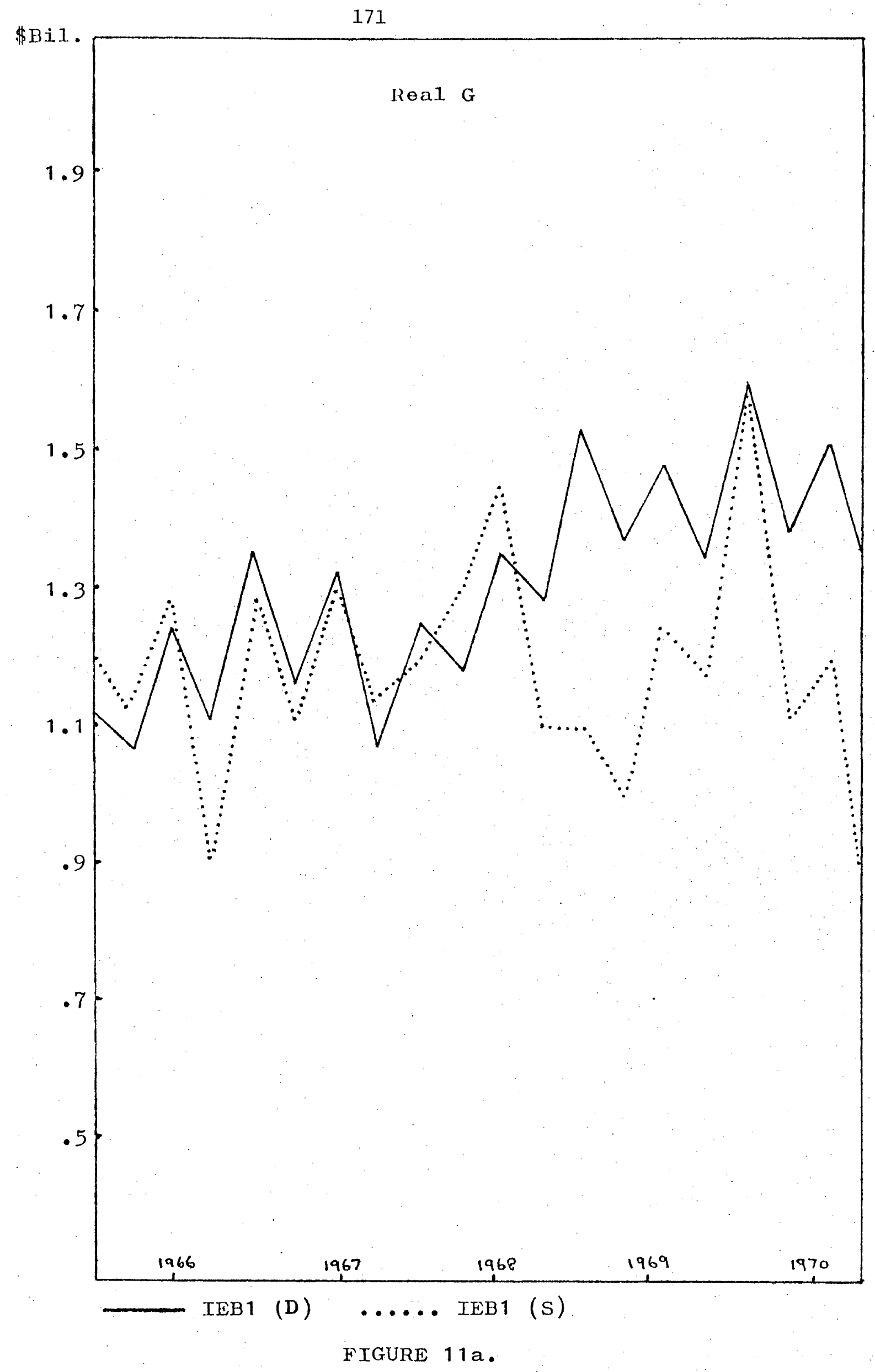


12 illustrates this proposition.) 20 If we believe that the model reflects the economy in a reasonably accurate way then it could be concluded that the government could have attained a more effective stabilisation of the balance of payments position over a twenty-period horizon beginning in the second quarter 1965. The historical results suggest that while monetary policy was moving in the correct direction, it was not manipulated sufficiently to produce results similar to those obtained in the IEBI experiment. How can we account for what appears to be a historically cautious use of monetary policy (in terms of the linear quadratic framework a "cautious" policy could be a policy resulting from a reasonably heavily weighted control)? One answer is that the authorities were unsure about the correct system structure but had a general idea of the size of policy impacts and it is equally conceivable, and indeed most likely, that it was not possible to obtain precise forecasts or current information about the uncontrollable exogenous variables. This leads to the not surprising result that, given better information about the system and the exogenous variables, the easier it becomes to stabilise the system. What is of interest however, is that even with a structurally estimated model and exact information on the uncontrollable exogenous variables, it may be possible to effectively fine tune the system within reasonable bounds given costs on the controls. This suggests that a substantial historical improvement in the performance of FR could have been obtained if the authorities had been willing to allow monetary policy to adjust in a more extensive manner. It cannot be emphasised too strongly that the above analysis depends on initially having a fairly accurate model. The model employed here obviously has weaknesses but if the policy multipliers are close to the "true" multipliers then the results and conclusions obtained from it could be regarded as fairly indicative of the true 


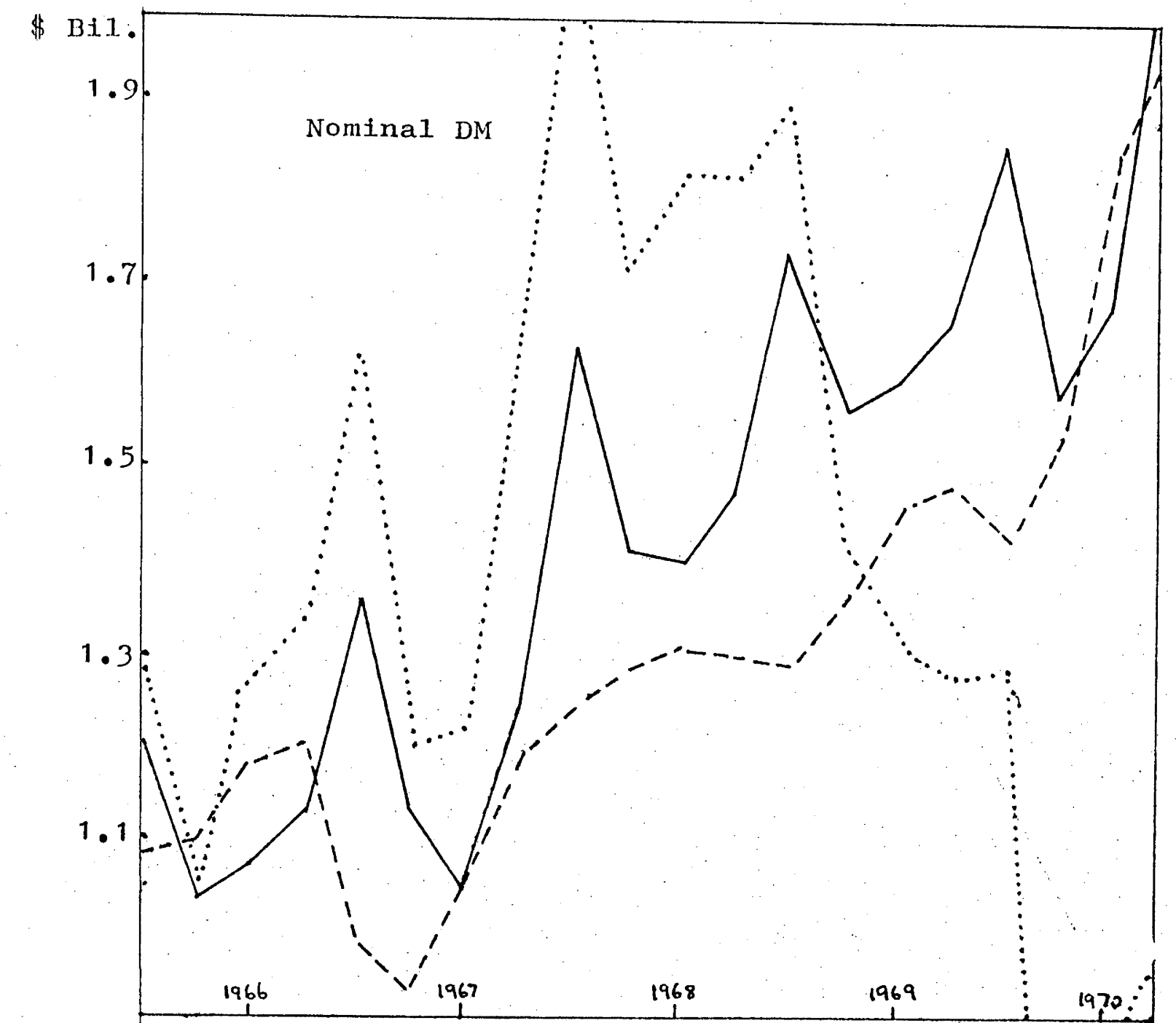

\$Bil.

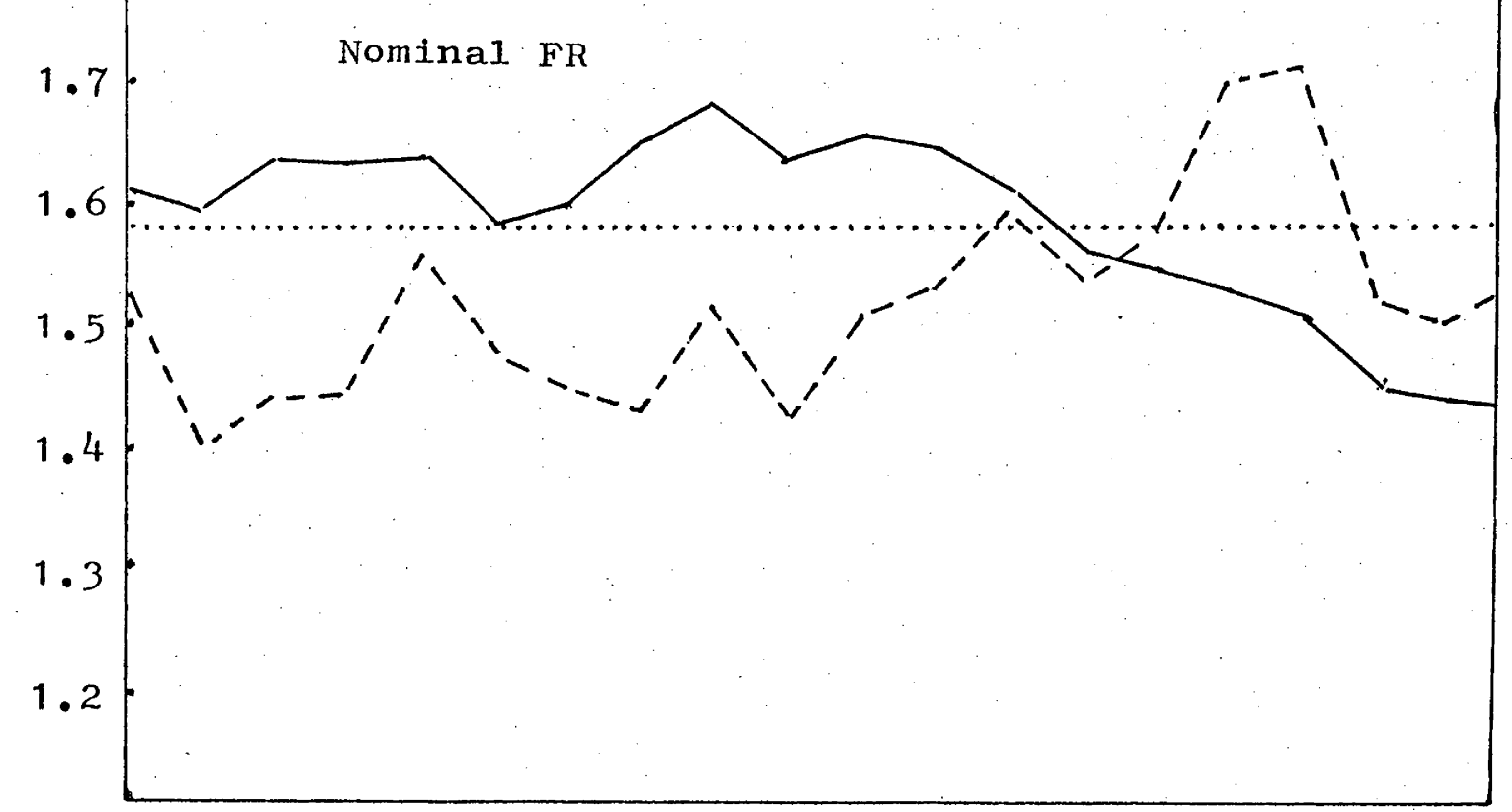

Historical 
performance.

The second experiment, IEB2, consisted of trying to exactly achieve internal and external balance regardless of where the system directs the other endogenous variables. In this situation we have two targets and two controls and provided that the appropriate rank conditions on the matrix of instrument impact multipliers is met, exact fine tuning can take place. If the instrument impact multipliers have rank equal to two then the problem collapses to a simple strongly-Tinbergen target path problem whereby the authorities can hit the desired targets in every period without resorting to anticipation or compromising the targets. The matrix of instrument multipliers relevant to the targets of $\mathrm{Y}$ and $\mathrm{FR}$ is given by

$$
\pi_{0}=\left[\begin{array}{lll}
2.1984 & & 0.1651 \\
0.0512 & \therefore & -.2547
\end{array}\right]
$$

and $\rho\left[\pi_{0}\right]=2$ hence the policy planner is indeed operating within a strongly-Tinbergen framework and optimisation within the linear/quadratic framework is redundant. As a point of comparison IEB2 was carried out in two ways. Firstly; the problem was solved as a dynamic fixed target problem with zero anticipation and secondly, it was solved within the linear/quadratic framework. It has already been pointed out that the linear/quadratic approach is unnecessary. However, it is of some interest to compare the two results, particularly in light of the fact that in the linear/quadratic framework some initial adjustment may take place as the time paths of the targets react to the initial conditions (therefore even with two targets and two instruments it may not be possible to exactly hit the targets until the system settles down) while in the fixed target framework the initial conditions are explicitly included in the solution. The fixed target problem is solved period by period while the linear/ 
quadratic control laws are solved initially over the entire planning period and in this respect may be computationally more efficient than the fixed target approach.

To enable the achievement of internal and external balance within the linear/quadratic framework, both controls were allowed to adjust freely. In terms of the cost function, $G$ and DM were assigned zero weights while $F R$ and $Y$ were weighted heavily using multiple weights to maintain the exact desired trade-off between targets. The relative weights assigned were

$\begin{array}{cccc}\text { Y } & \text { FR } & \text { G } & \text { DM } \\ 1000 & 1000 & 0 & 0\end{array}$

All other state variables were allocated zero costs and allowed to adjust freely. The results illustrate the fact that both solutions are identical (allowing for some insignificant deviations in the linear/quadratic case) which indicates that the optimally derived solution did not need to settle down as a result of the initial conditions. Recall that the targets for $\mathrm{Y}$ and $\mathrm{FR}$ are simple mappings of the initial conditions into target vectors. Limited as the evidence is, a tentative conclusion can be drawn along the lines that as long as the desired target paths are non-extreme mappings of the initial conditions, then the linear/quadratic solution will be identical to the fixed target solution (provided the number of targets and instruments are equal) for all time periods of the planning period. 21 The words "non-extreme" are very important qualifiers to the above statement and are best interpreted as meaning that economically meaningful targets are specified, for example, to specify a target of 108 annual real growth in $\mathrm{Y}$. from the appropriate initial condition for $\mathrm{Y}$ may require the system to settle down before the target is reached. 
As the results of the two solutions are identical, the discussion that follows can be regarded as being applicable to either technique. The results are graphed in Figures 8 to 11 . To keep real $Y$ on target required an expansionary fiscal policy, particularly towards the end of the planning period. This can be seen from Figure 10. Note that only the latter part of the optimal G has been graphed. This procedure was adopted to avoid confusion as the optimal $G$ for the first part of the planning period tracked between the optimal path of IEBI and the target. The need for strong fiscal policy stems from the fact that the target level of $G$ is not sufficient to sustain the target level of real $Y$. In addition, the optimal consumption path falls below its target. As consumption is the largest component of the national income identity, it is reasonable to expect that if it is declining then $\mathrm{Y}$ will tend to decline also unless strong fiscal action is taken to offset the movement in $C$. The divergent movement in the optimal paths of $\mathrm{Y}$ and $\mathrm{C}$ illustrates the important problem for applied control theorists already mentioned above, and that is the consistent specification of identities in terms of the components of a particular identity. It will be remembered that the target value of $Y$ was consistently constructed from the targets of the components of the national income identity. If all components are on target then $Y$ will be on target. However, as we have seen in the earlier simple example and in IEBI and particularly IEB2, the value of $Y$ that is chosen as a target will not necessarily be able to be achieved simultaneously with the target levels of its components. If policy planners are concerned about achieving target levels for all their income variables then high costs must be allocated to the appropriate variables in the cost function. 
the open and monetary sector variables (excluding FR of course) than the corresponding movement in income variables in the achievement of internal balance. The requirement that external balance be achieved implies that capital flows and the current account balance are equal. This has been achieved but at the expense of a stable total monetary base and a time path for the supply of money which exhibits more severe expansions and contractions from the path obtained in IEBI. To maintain external balance, monetary policy has been used more extensively than it was in the first experiment with the optimal path characterised by excessive expansions and contractions. Severe changes in monetary policy are necessary in order to maintain the equality between $\mathrm{CF}$ and $\mathrm{CB}$ and to ensure that $\mathrm{F}$. has a constant effect on the domestic economy. Even though FR is at its target level throughout the planning period it does have a varying indirect effect on the income sector through the excessive use of monetary policy required to keep FR on target. The effect of the monetary sector on $\mathrm{Y}$ does appear to be largely negated by fiscal policy with little in the way of excessive counteractive fiscal policy required to offset the monetary sector. This result is particularly encouraging as it indicates that the internal balance target can be achieved without excessive fiscal policy and without substantially disturbing the application of fiscal policy to account for monetary and external factors. On the other hand the severe stop-go monetary policy is a result not only of the requirements of the purely external variables and the uncontrollable exogenous variables which directly affect the external and monetary sectors; but is also a result of the fiscal policy necessary to achieve internal balance. This result occurs through the strong influence of the income sector on the open and monetary sectors, an influence which is not reciprocated from the open and monetary sectors to the income sector. 
This can be seen from the reduced form coefficients set out in Chapter Four. The monetary instrument not only has to contend with trade-offs in the non-income sectors but has to offset the influence of the income sector as well. The model is not sophisticated enough to reveal all the implications of a stop-go monetary policy and resulting behaviour of the money supply and interest rates. In a more complete model the presence of a severe monetary policy could have serious consequences for financial and asset markets. If so, then it raises the question of whether or not there should be a money supply or interest rate target incorporated into the list of major policy goals of governments.

In contrast to IEBl, interest rates each higher levels in the second experiment. The short rate is forced up by the increase in $Y$ relative to the first experiment and by the fact that generally there is not a large enough increase in $M$ to offset the influence of $Y$. The result is that by the end of the planning period the long rate has risen to 7.67. The higher levels of $\mathrm{RL}$ are not sufficient to generate a significant downward movement in $I$ as the strong fiscal action and high levels of $Y$ are able to keep real $I$ at a desirable level. As with IEBl, the high levels of RL over the last four periods have no influence on the system due to the lag structure but a significant downward shift in I could be expected in the next planning period.

Both monetary and fiscal policy have been strongly applied to achieve the desired targets. The requirement that both controls be allowed to move freely has meant that $G$ and. DM have been able to bear the burden of target achievement without excessive adjustment in all other non-weighted state variables with the possible exception of the total monetary base and the rates of interest. The variability in the total base and the rates of interest largely cancel out in the money supply 
function which accounts for the more subdued performance of $M$ in comparison with these variables. With so many income variables taking on the role of intermediate variables, comparatively little adjustment to complement the controls in the achievement of internal balance is required. The behaviour of the intermediate monetary variables is reversed however from the income variables with the requirement that they move excessively to complement the achievement of external balance.

The optimal stochastic solution results in neither internal or external balance being exactly achieved, which is to be expected. Fifty monte carlo simulations reveal that the optimal paths for both $Y$ and $F R$ track very close to their targets for all combinations of additive shock. An illustrative example is given in Figure 11. The optimal path for FR fluctuates about its target to a greater degree than real $Y$ but the shocks are not sufficient to move FR away from the general area of the target as was the case in IEBI (remember that identical shocks are used for the illustrative examples). The additive disturbances are not sufficient to shift real $Y$ substantially off target with the optimal stochastic path closely tracking its target and deterministic counterpart. The stochastic example is so close to its target that it was not possible to graph a sufficiently distinguishable path from the target in Figure 11 . The behaviour of the other major state variables is also similar to the deterministic case, allowing of course for the impact of the additive disturbances. The results clearly illustrate the self-correcting nature of the optimal control laws in the linear/quadratic framework to additive uncertainty. The fixed target solution is also self-correcting in relating to past shocks as $x_{t-1}$ is explicitly included when we solve for the appropriate values of the controls in each time period. It is worthwhile at this stage to re-emphasise a point made in Chaptex Two and that is that 
it is only in a strongly-Tinbergen world with as many instruments as targets that the dynamic fixed target approach to stabilisation will be able to adjust to additive disturbances from period to period.

The optimal stochastic paths of the control variables provide some interesting results. In the deterministic case strong expansionary fiscal policy was required to achieve the desired goals. The illustrative stochastic results (not graphed) indicate that under uncertainty this need not be the case. The optimal $G$ becomes closer to its target over the final periods of the planning period than the corresponding deterministic G. Monetary policy is once again of a stop-go nature but in the stochastic case it is more severe, particularly in periods fifteen to twenty. Although the direction of monetary policy has not been reversed, it is severely contractionary in the last five periods by comparison with the deterministic results and the target. More importantly, we have a switch in the optimal mix of monetary and fiscal policy from a situation of an expansionary fiscal policy in the perfect information case to a less expansionary fiscal policy and more severe monetary policy in the imperfect information case. The change in the mix of policy can be illustrated by the change in the RMSD value for both control variables when we shift from perfect information to imperfect information.

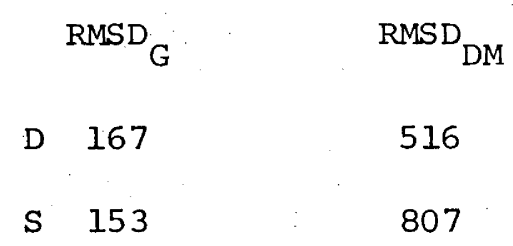

The letters $D$ and S refer to deterministic and stochastic solutions respectively.

The third experiment, IEB3, consisted of attempting to achieve internal and external balance as before, but in this case monetary policy 
was emphasised relative to fiscal policy. To achieve this policy mix a low cost was placed on deviations of DM from its target path while high costs were placed on $G, Y$ and FR. G,Y and FR were weighted such that a one percent deviation from the relevant target would have a thousand times the cost of a similar movement in DM. The relative weights were as follows

$\begin{array}{cccc}\text { Y } & \text { FR } & \text { G } & \text { DM } \\ 1000 & 1000 & 1000 & 1\end{array}$

The computer program accepts the relative weighting specifications and then generates actual weights which will maintain the desired relativity for all target values and all time periods. Once again we have a situation in which the number of targets equals the number of instruments but with the restriction that only one instrument can adjust in a completely free manner. This situation is not completely removed from the real world as it is not uncommon for governments to try and maintain a level of government spending to satisfy social and political needs and then rely on monetary policy to carry the burden of economic stabilisation.

The optimal deterministic paths for $\mathrm{Y}$ and $\mathrm{FR}$ indicate that given a restricted application of fiscal policy and a free application of monetary policy, the dual targets of internal and external balance cannot be achieved simultaneously (see Figures 13 to 15). Initially, real $\mathrm{Y}$ is very close to its target (the first four periods for $Y$ have not been graphed in Figure 13 to avoid unduly complicating the diagram) but as the planning period progresses it begins to fall just below target with the net result being an optimal path for $Y$ which is very similar to the corresponding path obtained in IEBI where $\mathrm{Y}$ and $\mathrm{G}$ were also weighted equally. In contrast to the behaviour of $\mathrm{Y}$, the optimal path of $\mathrm{FR}$ 


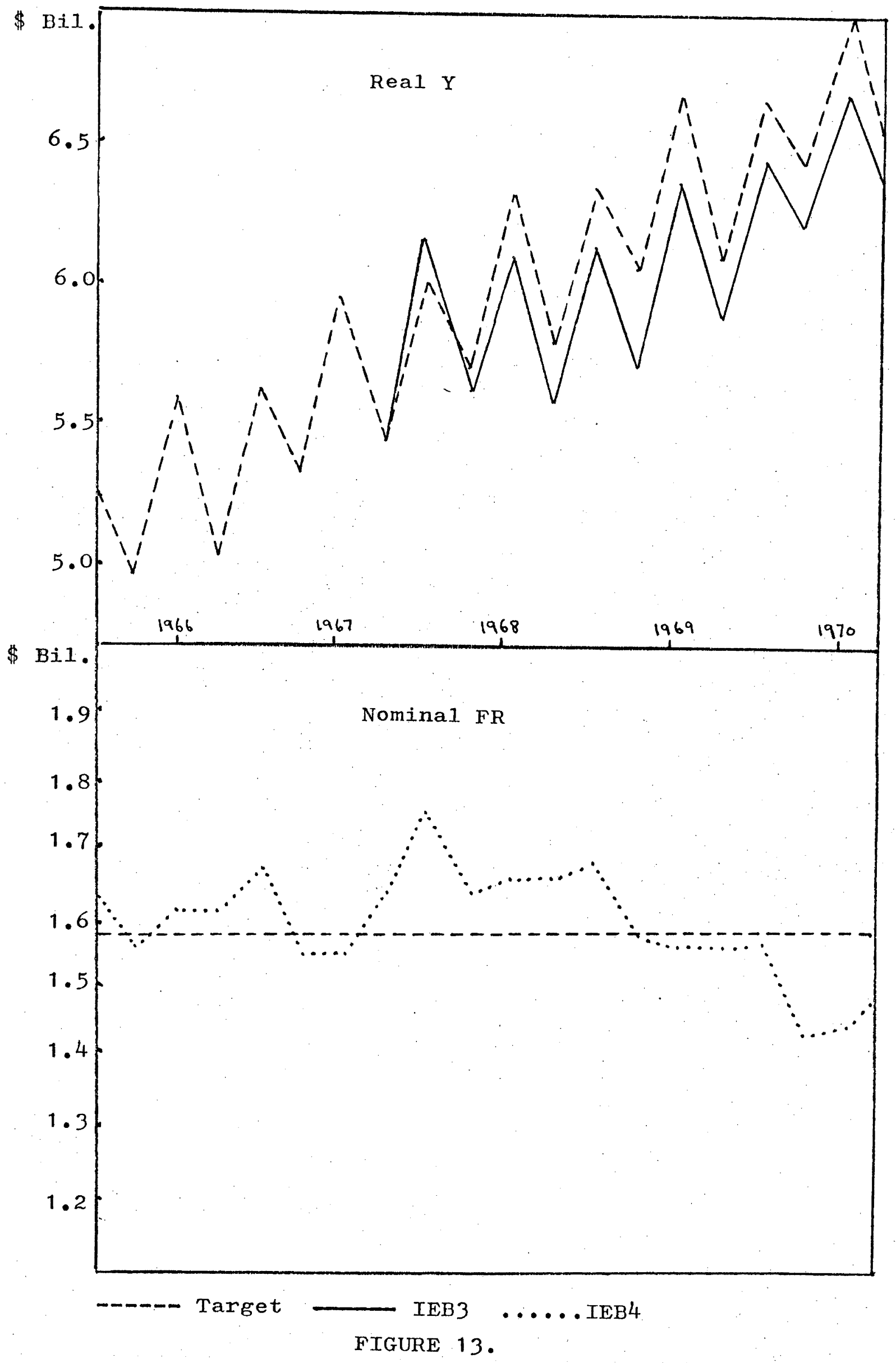




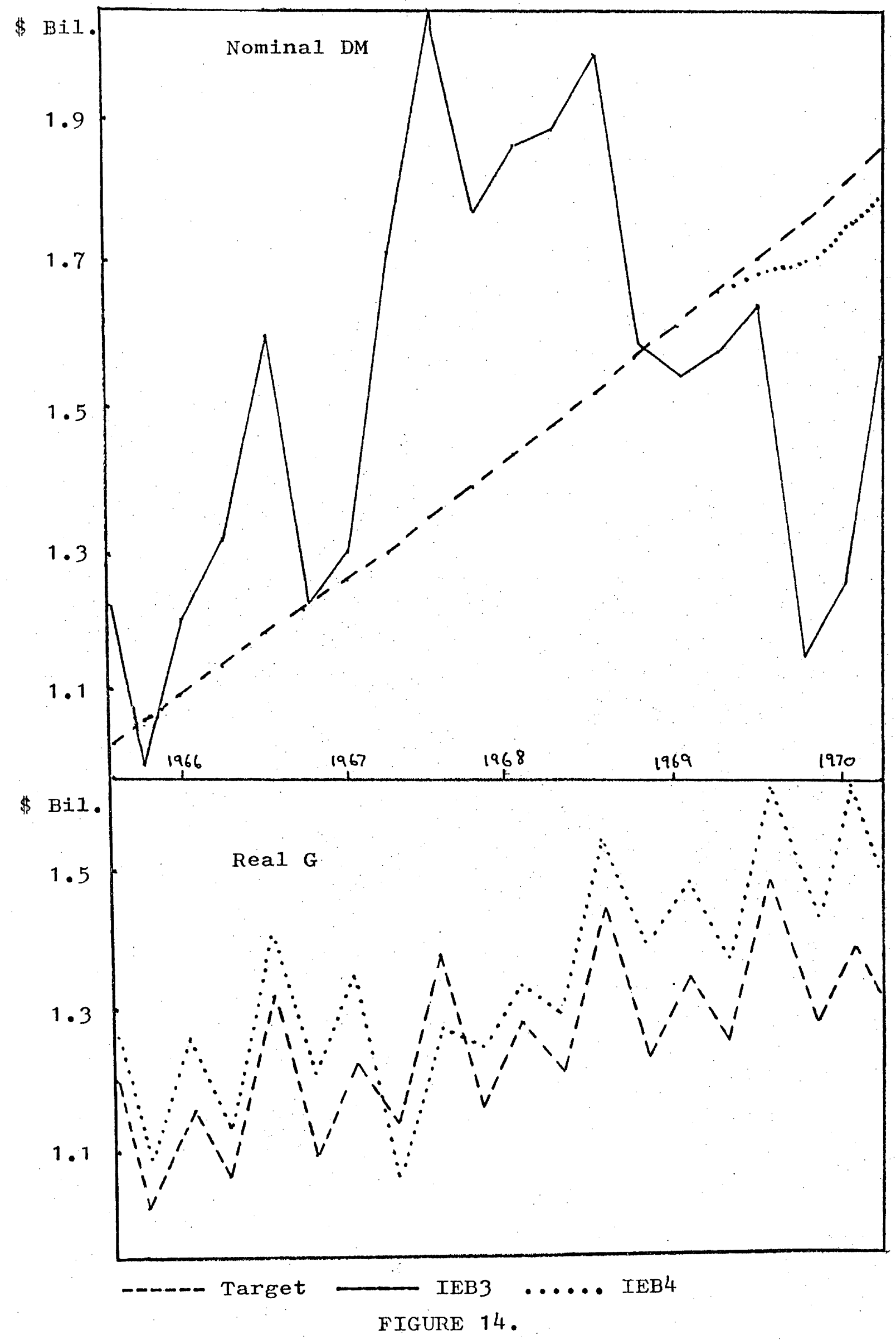




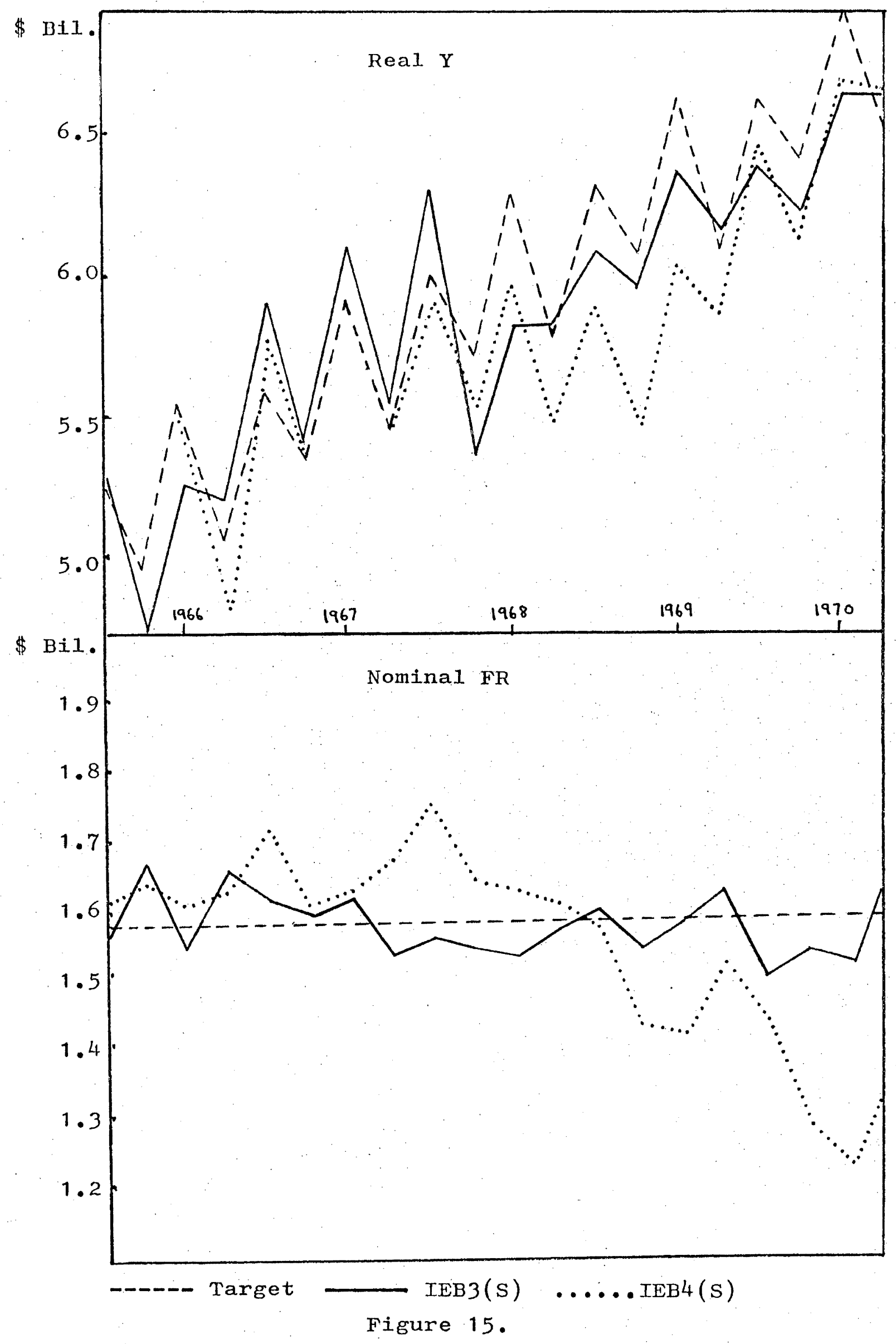


begins just below its target and gradually converges to its target path. The optimal trajectory for $F R$ is so close to its target that in a graphical form it is very difficult to distinguish it from the target and hence has not been graphed. FR performs better than $Y$ because the structure of the model is such that monetary policy has a limited impact. on $Y$ compared with its impact on the open and monetary sectors, $Y$ is only affected through a current low wealth effect on consumption and through the interest rate mechanism and then only with a lag of four periods. If $G$ is forced to follow its target path then it will force the income sector to move in accordance. The trade-off between the targets of $\mathrm{Y}$ and $\mathrm{G}$ that we have seen in IEBI and IEB2 will limit the ability of $G$ to exactly steer $Y$ on target under the current preference allocation but the general movement of the two variables will be similar. Monetary policy is virtually left free to offset the impact of $G$ on the external sector and to ultimately steer FR on target. To achieve external balance, $\mathrm{CF}$ must equal $\mathrm{CB}$. This condition is met at the end of the planning period although the two variables are not on target. A severe stop-go monetary policy is required once again although not quite as severe as the policy specified in IEB2. This is an interesting result as it indicates that the same target can be hit with the use of only one active instrument and the use of that active instrument is less severe than when both instruments are active. The reason for this lies in the fact that the difference in the use of $G$ from an active to a passive instrument when we shift from IEB2 to IEB3 has meant that the monetary instrument has not been required to offset any strong bursts in fiscal policy in order to stabilise the external. sector. By comparison with IEB2, the optimal monetary policy required in this case becomes much more expansionary at the end of the planning 
period (it is still contractionary in relation to the desired time path). The resulting mix of policies is a combination of fiscal policy which is close to its target and a monetary policy which is less severe over particular periods than the corresponding mix in IEB2 where both controls were active, that is, allowed to adjust freely. To some extent this result is counter-intuitive as one would generally expect that monetary policy would need to be more severe to take account of the passive nature of G. The analysis outlined above gives some insight into why this may occur. Another point to consider when attempting to analyse why a particular policy is severe or not is the degree of consistency between targets. A set of very inconsistent targets may well produce a severe monetary policy under the above conditions. Before discussing the stochastic results it should be noted that while FR performs better than $\mathrm{Y}$, the degree of superiority of performance is only marginal and indeed $\mathrm{Y}$ has been effectively stabilised close to its target which is not particularly surprising considering that it was weighted so heavily in the cost function.

The stochastic solutions to IEB3 provide some very interesting results. The behaviour of FR differs considerably from the deterministic solution. See for example the illustrative case in Figure 15. However, as in IEB2 the stochastic path of FR fluctuates about the target and any shocks which tend to move FR off target are compensated next period by the movement of DM. A close comparison of the optimal stochastic paths of FR for both IEB2 and IEB 3 reveals that they are almost identical in all of the fifty monte carlo solutions (remember that identical random shocks were used for each IEB experiment). The example graphed in Figure 15 shows that the optimal trajectory for $\mathrm{FR}$ in IEB3 was only marginally more off target in particular quarters than it 
was in IEB2. This suggests that the monetary instrument, was adjusting almost exclusively to the uncertainty present in the open sector. The optimal path for $Y$ tracked reasonably close to its target and in some instances performed better than the deterministic solution. Figure 15 illustrates a situation in which the stochastic solution tracked close to its target and indeed, toward the end of the planning period, became slightly closer to the target than the deterministic solution. Not too much should be made of this difference as in both cases the differences between the target and optimal path are minimal and are differences which would certainly be disregarded by governments who would most likely claim to have implemented a successful stabilisation program based upon the results obtained here.

The stochastic performance of $Y$ appears to be counter-intuitive as it would be expected that given a restricted use of fiscal policy, shocks to the system would produce significant fluctuations in $\mathrm{Y}$. It must be concluded, at least in relation to the illustrative example, that the shocks have been complementary to $G$ in relation to stabilising $Y$. However, as a general safety first rule, to reduce the impact of uncertainty, the controls should be allowed to adjust more freely than would be the case under perfect information. The larger the shocks the less restriction there should be on the controls and while it has been illustrated that shocks can be stabilising, it is generally not always possible to know the size and direction of shocks beforehand. If some attention is not paid to the effects of shocks then the system may not move in the desired direction.

As could be expected, the optimal stochastic paths of $G$, both real and nominal are very close to their targets. This is due to the high costs allocated to $G$ and because $G$ is not subject to direct 
shocks itself. The failure of $G$ to adjust to the shocks in the system, particularly in the income sector, is a direct result of the nature of the feedback matrices, $F_{t}$ ' and vindicates to some extent the comment contained in Chapter Two of the direction in the movement of the feedback coefficients when costs on controls are increased relative to other costs. Table 6 lists the feedback matrices for selected periods for IEB3. 22 When assessing the impact of feedback effects one should bear in mind the relative magnitudes of the variables of concern. (See the' nominal target paths set out in Chapter. Four and the graphs presented in this chapter for an indication of relative magnitudes.) The important point is that the increase in costs on $G$ in IEB3 compared with IEB2 has meant that the optimal $\mathrm{G}$ in any period is less responsive to lagged values of the income variables and $F R$ and hence will be less responsive to past shocks to the income sector and open sector than was the case in IEB2. In IEB2, G was exceptionally responsive to past shocks. It will also be noticed from Table 6 that while the income coefficients for IEB3 relevant to $G$ have declined in relation to IEB2 (coefficients for: $\mathrm{Y}_{t-1}=.17, \mathrm{C}_{t-1}=-.12$, $I_{t-1}=-.52$, for IEB2 linear/quadratic solution), the monetary coefficients, in particular the lagged interest rates, (with the exception of $\mathrm{RS}_{\mathrm{t}-1}$ and $R L 3_{t-1}$ ) have increased making $G$ more responsive to those variables (coefficients for: $\mathrm{FR}_{\mathrm{t}-1}=-.38, \mathrm{RS}_{\mathrm{t}-1}=-22.61$, for IEB2 linear/quadratic solution). However, as the shocks to those variables are minimal in relation to $Y$, little adjustment is produced in $G$ when we move from a deterministic to a stochastic situation. The major contribution to the formation of $G$ comes from the additive vectors $f_{t}$. In contrast to $G$, it can be seen that DM remains just as responsive to all lagged variables 


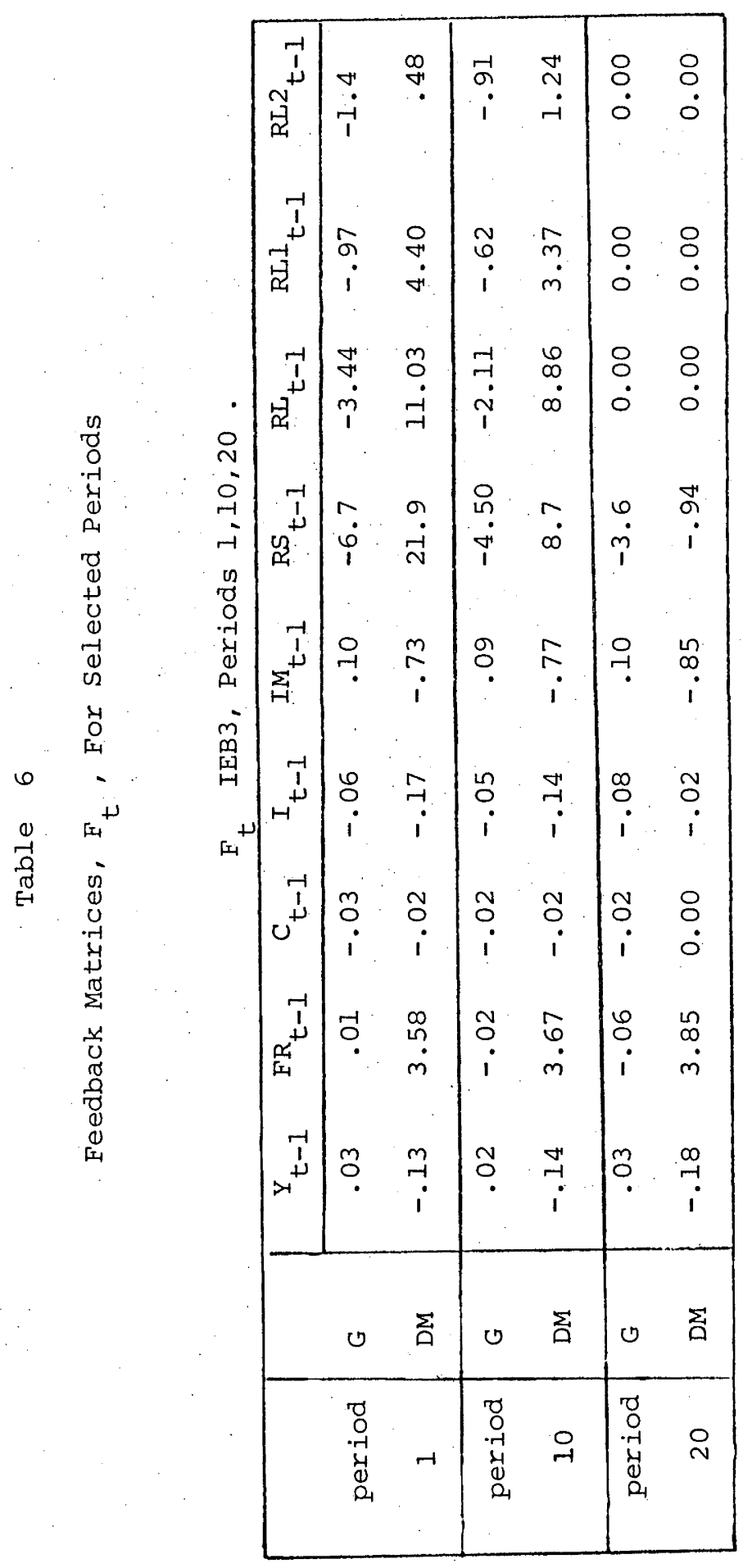




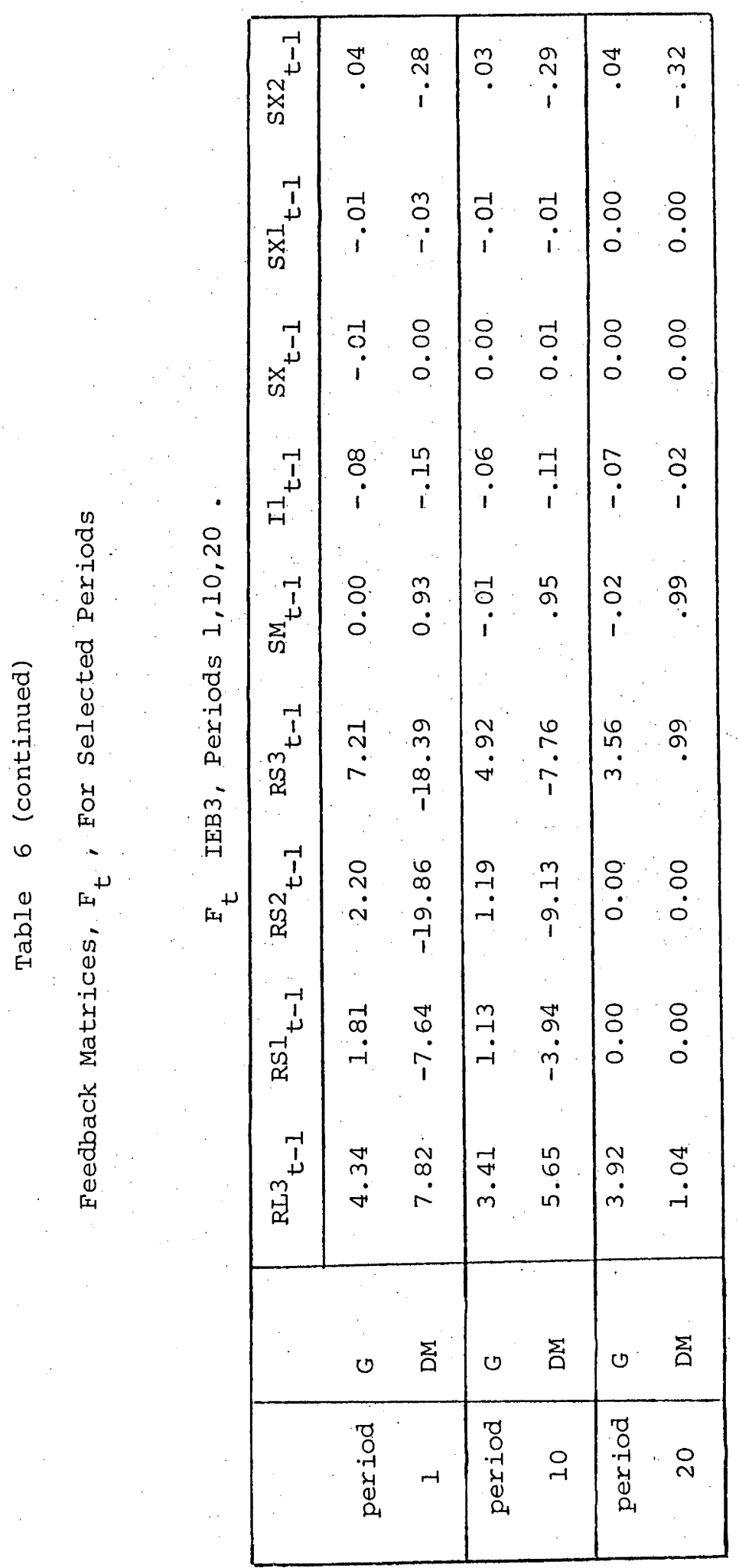


as it was in the corresponding IEB2 case. Note that in the fixed target solution to IEB2, all lagged variables play an integral part. Of particular interest is the dominance of lagged FR in the feedback matrix thus explicitly illustrating why DM adjusts more readily to shocks in the open sector at the expense of the other sectors. The importance of $\mathrm{FR}_{t-1}$ in the feedback matrix also ensures that monetary policy is predominantly assigned to external balance and illustrates how the linear/quadratic procedure effectively employs all information within a reduced form to make a correct policy assignment.

Like G, DM is not subject to any direct shocks itself (by direct shocks we are referring to structural shocks associated with particular variables), yet its behaviour is strongly influenced by the shocks to the endogenous variables of the system as it is free to adjust in accordance with the requirements of the rest of the system, in particular in relation to $F R$ as has been demonstrated above. In comparison with the deterministic case and the target, period 13 onwards is a period of strong contractionary monetary policy. A similar situation occurred in IEB2. However, in that case $G$ was allowed to adjust freely as well and thus was able to take away some of the emphasis on monetary policy. In IEB3 $G$ is unable to adjust so a more severe monetary policy is required to try and maintain the system objectives. This was true for all monte carlo repetitions as well as the illustrative example. The presence of uncertainty in the illustrative example and other unreported stochastic solutions, almost resulted in a reversal in the direction of monetary policy in the last few periods of the stochastic case as compared with the deterministic case. The possibility of this situation occurring suggests that serious consequences could arise for policymakers. In particular, the optimal mix of policy in both cases highlights 
the problems that could result if policy is formulated on the basis of perfect information when in fact, because of uncertainty, an opposite or more severe policy is required to achieve the desired trade-off between objectives. The effects of applying an inappropriate policy are only too well known. The shift in the use of monetary policy from IEB2 to IEB3 (deterministic and stochastic) is illustrated by the appropriate RMSD's as follows:

$\begin{array}{cccc} & & \text { RMSD } & \\ & & & \\ & \text { IEB2 } & & \text { IEB3 } \\ & & & \\ \text { D } & 516 & & 378 \\ \text { S } & 809 & & 860\end{array}$

where the letters $\mathrm{D}$ and $\mathrm{S}$ refer to deterministic and stochastic respectively. Note that the deterministic DM in IEB3 was less severe than IEB2 while the stochastic DM in IEB3 was more severe than IEB2.

For the fourth experiment the emphasis on the control variables was reversed from IEB3. In IEB4, $G$ was allowed to adjust freely while DM was forced to track closely to its target path. As before, high costs were placed on $Y$ and $F R$ and zero costs were assigned to all other state variables and as was the case with all other experiments, multiple cost matrices were used throughout the planning period in order to maintain the desired trade-offs. The relative weighting position (as distinct from the actual weights) was specified as,

$\begin{array}{cccc}Y & \text { FR } & \text { G } & \text { DM } \\ 1000 & 1000 & 1 & 1000\end{array}$

The requirement that $G$ be used extensively rather than DM produced an optimal solution whereby both targets of internal and external balance were not simultaneously achieved. The deterministic results 
somewhat surprisingly reveal that the optimal path for $Y$ was almost identical to that obtained in IEB3. The closeness of the overall result is indicated by the respective RMSDs which are 146 for IEB 3 and 144 for IEB4, a negligible difference. The achievement of similar RMSDs was not due to the fact that one or two opposite and extreme levels of $\mathrm{Y}$ counterbalanced the other values to give the impression of an overall closeness of performance, but was a direct result of both paths being almost identical. The closeness of the two paths is illustrated by the fact that they could not be easily separated graphically and hence real y, IEB4, has not been included in Figure 13. A strong burst of fiscal policy towards the end of the planning period is not sufficient to exactly achieve the internal balance target over the same period although it must be conceded that real $\mathrm{Y}$ has been effectively stabilised at an acceptable level. Real consumption lies below its target as does total personal income and real disposable income. The increasing paths of $\mathrm{Y}$ and $\mathrm{G}$ are not sufficient to keep real consumption on target. Because of the simultaneous nature of the model, the failure of $C$ to reach its target in turn helps prevent $Y$ from consistently reaching its target. The optimal paths of $\mathrm{Y}$ and $\mathrm{G}$ are sufficient however to generate an optimal path for real I which exceeds its target, even though interest rates are increasing severely over time. It should be noted that a strong movement in investment is not sufficient in itself to sustain a corresponding movement in real $\mathrm{Y}$. Complementary movements must take place in consumption and government spending.

The behaviour of $\mathrm{FR}$ is not as acceptable as that of $\mathrm{Y}$. It is apparent from the three preceding experiments that strong monetary policy aimed specifically at the external sector is required to achieve external balance. In this case, the requirement that DM follows its target 
implies that the authorities are aiming for a smooth domestic monetary expansion. In relation to the target of external balance, this is a passive policy and the results clearly show (see Figure 13) that it is incompatible with external balance. A smooth increase in domestic base money will have a continual negative impact on capital flows. An easy domestic monetary situation will mean that less foreign capital will be required. At the same time the optimal paths of $\mathrm{Y}$ and $\mathrm{G}$ generate an increasing time path for imports, thus upsetting the balance between capital flows and the current account balance. IEBI indicated the difficulties in moving $F R$ back on target after it had moved off. The results from this experiment help to confirm that unless corrective monetary action takes place, severe undesirable fluctuations in foreign reserves will continue to occur.

The optimal path for DM does keep relatively close to its target except for the last few time periods where there is a slight deviation which suggests that as $\mathrm{G}$ is unable to keep both variables on target without policy anticipation. Without some movement in DM to complement $G$, the welfare costs associated with FR would have been exceptionally high. Even with the slight deviation in DM from its target the welfare costs compared with IEB3 are very high

$$
\begin{aligned}
& \text { IEB 3 IEB4 } \\
& \begin{array}{lll}
\text { welfare cost (D) } \quad 78.4 & 195.8
\end{array}
\end{aligned}
$$

It can be seen from the above figures that even in a deterministic world, an attempt to achieve both targets without anticipation will result in a welfare loss of nearly $300 \%$ from the case where DM adjusts freely to the case where $G$ adjusts freely. This suggests that if a policy is to be passive then that policy should be fiscal policy as the results of IEB3 
compared with IEB4 show that an excellent degree of stabilisation can be achieved by the use of monetary policy alone provided that the desired paths for $Y$ and $G$ are growing at approximately the same rate and there is an absence of severe price inflation. The increase in the value of the cost function from IEB3 to IEB4 is due almost entirely to FR as $Y$ is virtually identical in both experiments.

Because of the trade-off between. DM and FR, the smooth expansionary action of DM is offset and upset by FR. As a result the total monetary base and the money supply fail to follow a smooth expansionary time path. The time path of the money supply is further complicated by the short rate of interest which influences the money supply directly and at the same time is influenced by the money supply. The smooth expansion of DM, along with $\mathrm{RS}$, produces a reasonably smooth expansion in $M$ for the first half of the planning period. After period ten, the money supply begins to contract and does not return near to its target until the end of the planning period. The contraction is initially brought about by a fall in the short rate of interest generated by a decline in the demand for money (reflected in the nominal level of $\mathrm{Y}$ ) relative to money supply. This type of behaviour was also present in IEB3 but was more severe in the third experiment than the fourth. The shift in the emphasis of the intermediate monetary variables between IEB3 and IEB4 can be illustrated by the relevant RMSDs

$\begin{array}{cccc} & \mathrm{RMSD}_{\mathrm{M}} & \mathrm{RMSD}_{\mathrm{RS}} & { }^{\mathrm{RMSD}}{ }_{\mathrm{RL}} \\ \mathrm{IEB} 3 & 703 & 1.80 & 1.27 \\ \text { IEB } 4 & 362 & 1.71 & 1.23\end{array}$

The differences between the rates of interest are negligible while it is apparent that there is a $50 \%$ improvement in the overall performance of $M$ 
from IEB 3 to IEB4. Even though there is little change in the performance of the rates of interest, their actual values may be of some concern as in both cases the levels become high towards the end of the planning period.

It appears that the goals of internal and external balance cannot be exactly achieved in an optimising framework if one instrument is restricted (although $Y$ has been effectively but not exactly stabilised in all experiments), especially if the restricted instrument is the monetary instrument. As effective as fiscal policy is, it is not sufficient by itself (unless perhaps a fixed target approach is adopted) to move the economy in the desired direction. An appropriate mix of policy is required.

The stochastic solutions provide little additional information about the behaviour of the state variables and the trade-off between $Y$ and FR. $Y$ performs in a manner similar, in the illustrative example, to the deterministic case with the exception of a reasonably large contraction in periods twelve to sixteen (see Figure 15). The fact that $G$ does not move to offset the shocks which contribute to the short contraction is an indication that, unlike DM in IEB3, G adjusts in response to movements in both targets rather than being predominantily assigned to $Y$. The coefficients of the feedback matrices presented in Table 7 confirm this assertion. An interesting feature of the feedback coefficients is that while $G$ responds to both income and external/ monetary variables, the response to $\mathrm{FR}_{t-1}$ declines towards the end of the planning period which is partly a result of scaling the cost weights to avoid cost function bias but at the same time the coefficient for DM increases over time to a point where the coefficient relating $F_{t-1}$ to current $G$ and $D M$ is virtually equal in absolute terms (with different signs). The equality of the size of the coefficients accounts 


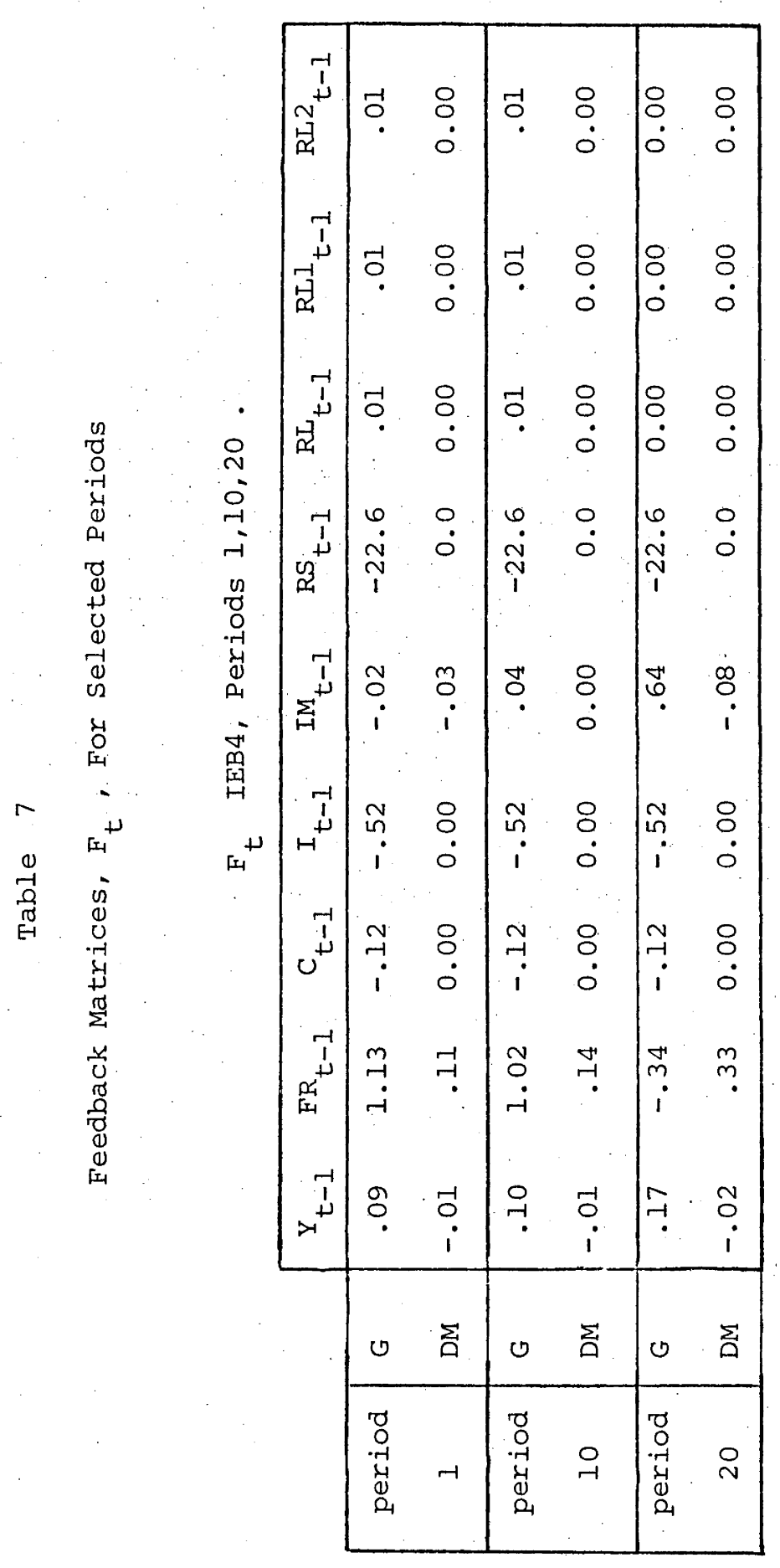




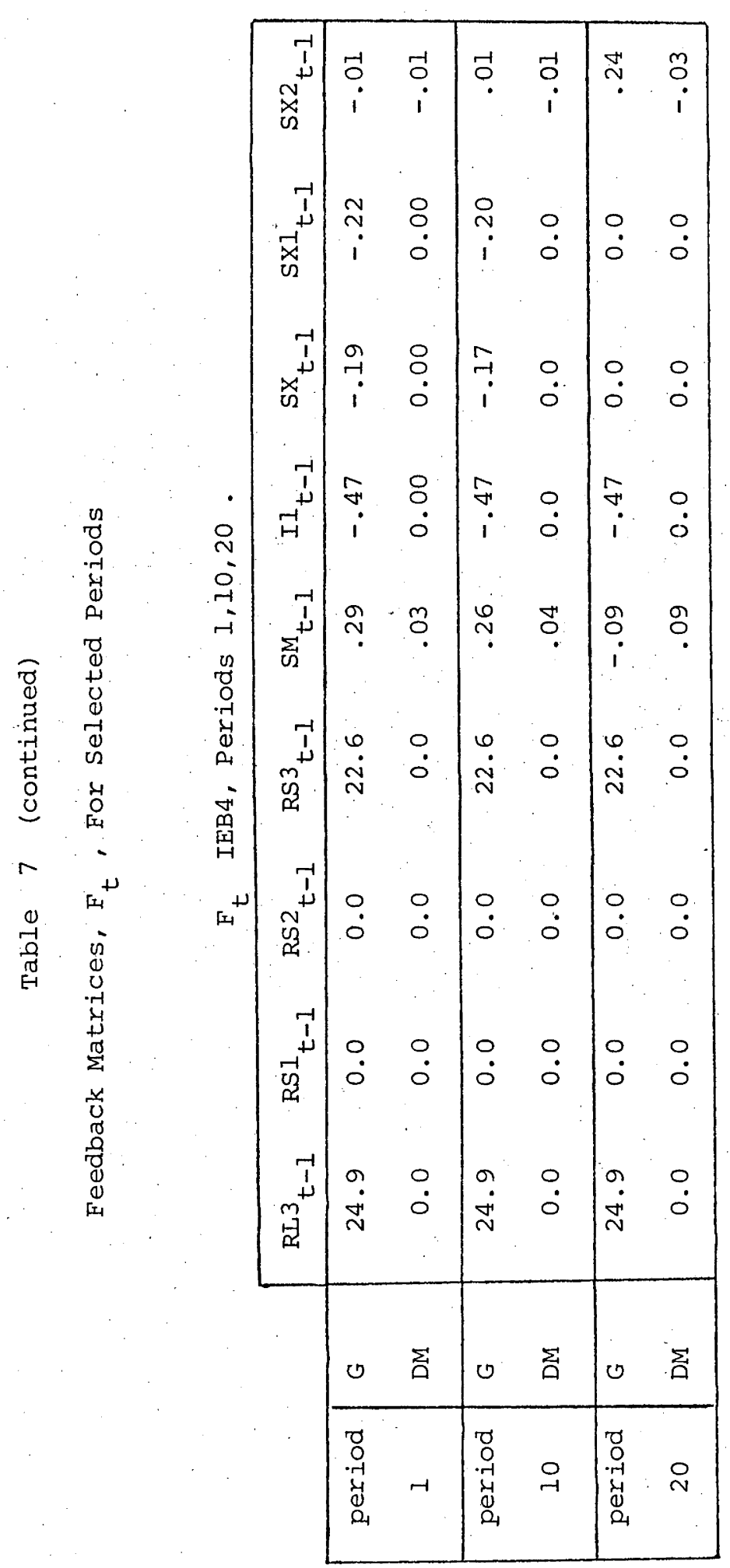


substantially for the deviation of DM from its target towards the end of the planning period in the deterministic solution and the even greater deviations found in stochastic solutions. The response of $G$ to both targets results in a severe movement in $G$ in the illustrative stochastic solution (as it did in other unreported experiments). The RMSDs listed below indicate the shift in policy from deterministic to stochastic.

\begin{tabular}{ccc} 
& RMSD $_{\mathrm{u}}$ & $\mathrm{RMSD}_{\mathrm{DM}}$ \\
$\mathrm{D}$ & 95 & 10 \\
$\mathrm{~S}$ & $\vdots$ & 65 \\
\hline \% Change & 240 & 600
\end{tabular}

The shift in the use of DM must be viewed with caution as the high percentage change is somewhat misleading. The high percentage change is due to the fact that DM showed very little target deviation in the deterministic case. Nevertheless, DM was forced to deviate under uncertainty but compared to $G$, the deviation of $D M$ in the stochastic case was minor. The deviation of DM. from its target in the stochastic solution IEB 3 solution was of a similar percentage difference to the deterministic case as was the situation for $G$ in IEB4 but it can be seen from the feedback coefficients and results for IEB3 that the adjustment was largely as a result of the external sector only. Towards the end of the planning period $G$ declines relative to the deterministic case with the shocks once again being complementary to $G$ in respect to stabilising the income target at a position close to its desired path. It has already been pointed out that DM was forced to move off target in the stochastic solutions and indeed in the illustrative example DM was subject to a noticeable contraction towards the end of the planning period. The 
important result arising from the stochastic solutions is that the more vigorous use of fiscal policy required to maintain internal and external balance in the absence of policy $\mathrm{mix}$ from the deterministic case which is able to keep real $Y$ moving in the right direction but at the expense of the external balance target.

SINGLE TARGET ACHIEVEMENT AND A FURTHER COMMENT ON INSTRUMENT INSTABILITY

The preceding experiments show that the type of unstable behaviour predicted by the instrument instability test for one target, does not necessarily exist when an additional target is introduced and the instruments are required to trade off targets. Of course this result will not hold for all models and all targets but it does suggest that it is possible that even though the underlying structure of the system predicts a rapidly increasing or decreasing time path for a particular instrument aimed at one specific target, the need to trade-off targets and to offset exogenous influences may result in the explosive tendency being neutralised, eliminated or swamped by the additional requirements of the system and instruments. As such, the underlying instrument instability associated with a particular target will not be of any concern to the policy-maker from the point of view of taking some corrective action in the future to prevent an adverse response by the private sector manifested in detrimental changes in expectations and undetected (by the government) structural shifts, for example.

The single target instrument instability tests discussed in the preceding chapter indicated that fiscal policy directed specifically towards the external balance target with DM fixed at its desired level, resulted in an unstable income sector which fed back into the open sector producing a destabilising influence thus requiring unstable compensatory 
responses by $G$ which generated further instability. The identical instrument weightings used in IEB 4 along with the inclusion of $\mathrm{Y}$ in the cost function with FR has removed this unstable instrument behaviour. Similarly, DM directed specifically at $Y$ produced instrument problems which were subsequently eliminated by the inclusion of an additional target. DM also illustrated an explosive tendency when aimed at FR alone (due to the influence of $\mathrm{CF}$ ). This explosive tendency was not detected in the four preceding experiments although it must be conceded that the inclusion of the historical exogenous variables may have been an important offsetting factor. Thus, an appropriate selection of multiple targets can eliminate single target instrument instability as distinct from Holbrook's original concept of instrument instability and Turnovsky's later analysis within the linear/quadratic framework.

The problem of hitting a single target will be examined further within the context of external and internal balance. The experiments discussed will differ from the earlier single target experiments in that target paths growing over time are assigned to $G, Y$ and DM as distinct from constant targets and the historical exogenous variables are included. The experiments are exercises in exact fine tuning and examine in turn the effects of hitting either the external balance or internal balance target on the remainder of the system and also examines whether or not undesirable instrument variations occur within a more realistic framework than the framework initially employed in the single target experiments. The relative cost function weightings for the six experiments are presented below. As before, multiple cost matrices were used to eliminate cost function bias. 
Table 8

IEB5 - IEB10. Relative Cost Weights

\begin{tabular}{lcccc} 
& Y & FR & G & DM \\
\cline { 2 - 5 } IEB5 & 1000 & 0 & .1 & .1 \\
IEB6 & 1000 & 0 & 1000 & .1 \\
IEB7 & 1000 & 0 & .1 & 1000 \\
IEB8 & 0 & 1000 & .1 & .1 \\
IEB9 & 0 & 1000 & 1000 & .1 \\
IEB10 & 0 & 1000 & .1 & 1000 \\
& 0 & & &
\end{tabular}

Small positive costs are associated with the controls when both controls are freely adjusting to overcome the problem of a lack of uniqueness if zero instrument costs were allocated. Small costs were associated with the free instrument when the other is fixed to ensure the exact freedom of adjustment between experiments and to avoid any rank failures which may occur through computational rounding and so on. RMSD results for selected state variables and the instruments are given in Table 9 while selected time paths for $\mathrm{Y}$ and $\mathrm{FR}$ are presented in Figure 16.. Note that the RMSDs cannot be compared between different variables due to different scaling and can only be compared between different experiments for the same variable. The first thing to be noted is that DM should not be assigned to the income target. The RMSD for DM indicates the severity of the policy required which produces the worst overall result for $M B, F R$ and $M$ for the six experiments. The interesting factor is that monetary policy is unstable even though fiscal policy follows its target exactly. We have seen above that if fiscal policy follows its target then $\mathrm{Y}$ will be stabilised very close to its desired path. To close the gap between $Y$ and i.ts target requires an unstable monetary 


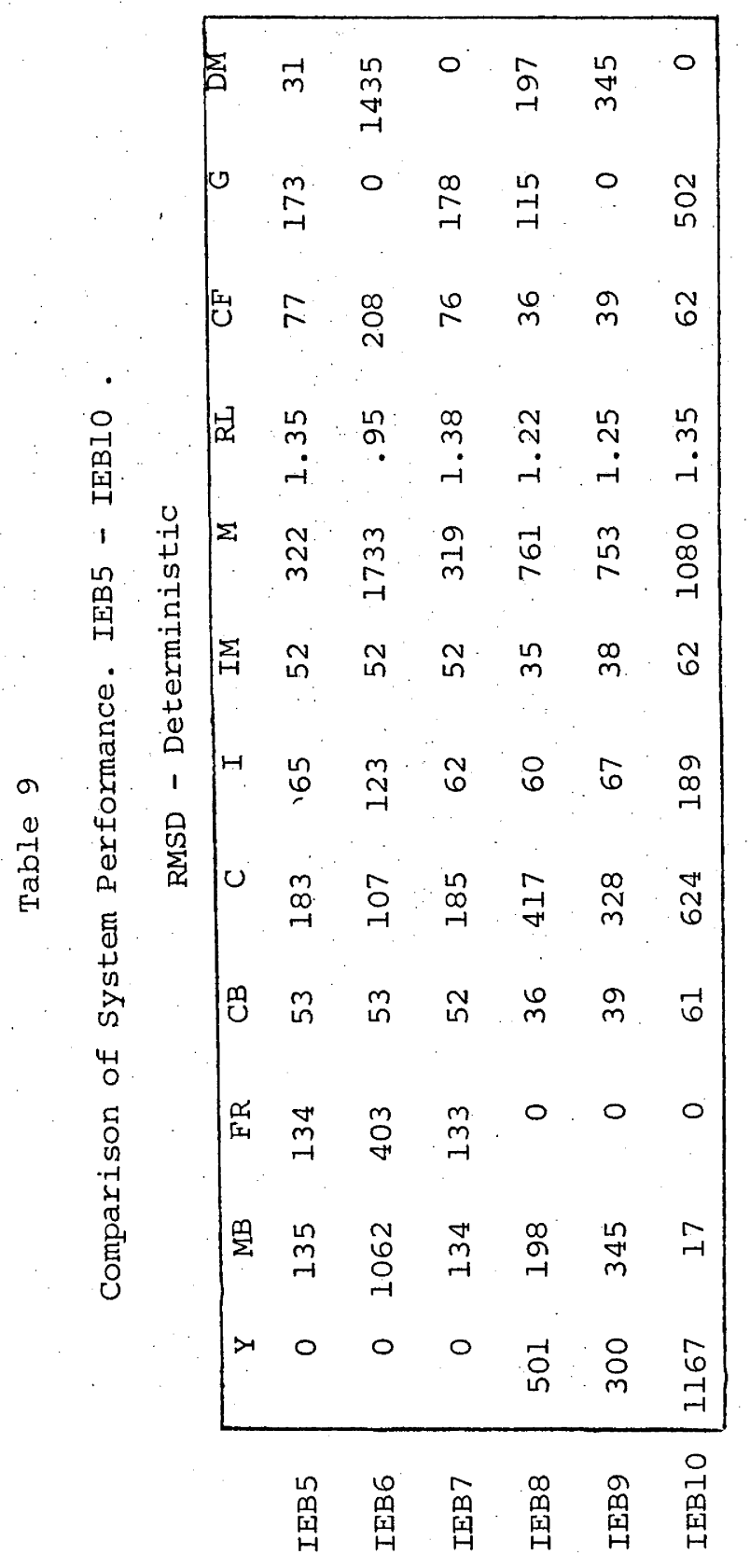




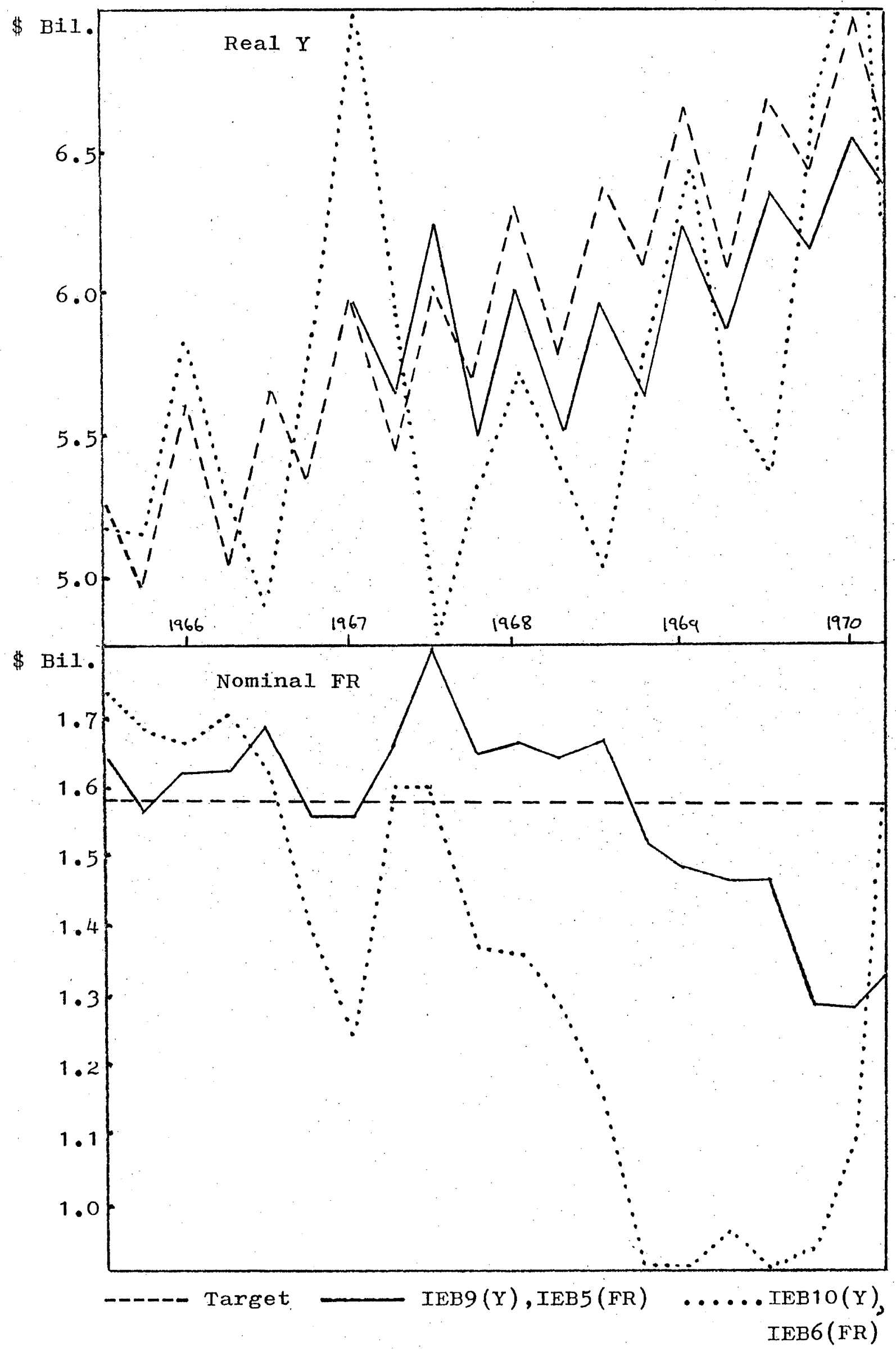

FIGURE 16. 
policy, that is, a small movement in $Y$ requires an exceptionally severe movement in DM. On the other hand fiscal policy should not be exclusively directed towards external balance when the domestic monetary base is allowed to follow a smooth time path. These results are perhaps not surprising as they reflect the concept of effective market classification put forward by Mundell (1962) some time ago and do not differ substantially from the results obtained in the single target instrument instability analysis. The achievement of both $\mathrm{Y}$ and FR individually using both instruments produces a feasible mix of policy with very little monetary policy being used to assist $G$ in steering $Y$ to its target while both instruments are used extensively to achieve the FR target. Reasons for this can be found in the reduced form where it can be seen that $G$ strongly influences all sectors of the system as opposed to the limited impact of DM. Of the six experiments only four can be regarded as feasible in terms of the required policy mix. These are IEB5, IEB7, IEB8 and IEB9. A brief comment on particular policy specifications in light of the results obtained here and the earlier stability results is warranted. Firstly, the optimal monetary policy required in IEB9 is of a severe nature but feasible, with a time path that appears to be somewhat oscillatory. The earlier stability result for the identical cost function specification indicated a severe, almost explosive upward movement in DM. Can the two be reconciled? The answer is yes when it is remembered that in IEB9 exogenous variables are included not only directly in the FR relationship but also in the variables that feed back into $F R$ and in addition $G$ is following an increasing rather than constant time path. The net result is that the optimal monetary policy indicates that DM is not explosive when coupled with $\mathrm{FR}$ when in fact the underlying structure of the system indicates that DM may be explosive. The implications of 
this are only too clear. An incorrect assessment of the stability properties of the system by the policy authorities could lead to an inappropriate cost function weighting. For example, the monetary policy of IEB9 might suggest that DM is not unstable and therefore no corrective action is required. However, an easing in the volatile nature of the uncontrollable exogenous variables in the future may allow the structure to dominate the exogenous variables in the sense that the underlying explosive nature of DM will emerge. The authorities may regard this as a short run perturbation and not take any action. Realisation of the problem may not occur until it is too late due to the original misinterpretation of the dynamic stability properties of the instrument. The second set of important results concerns IEB10 in which the most severe fiscal policy is employed. The optimal $G$ obtained in this experiment is one in which the time path fluctuates severely in a manner which should not be acceptable to any planning authority. However, in the earlier stability experiments it was claimed that given the same cost weightings as IEBIO and constant targets and constant exogenous variables, the optimal fiscal policy exhibited a tendency to move in a significantly severe downward direction. In this case we have a situation in which the underlying tendency of the instrument to seemingly explode downwards has been replaced by a severely fluctuating time path. The conclusion to be drawn here is that the instability has in this case been generated solely by the interaction of the exogenous variables and the requirements of the desired time path for FR and is not a function of the underlying structure. It is clear then that the presence of exogenous variables in the system can lead to incorrect conclusions about instrument instability properties of the system unless a formal analysis is pursued or the exogenous variables have very little impact or are held constant. Although the situation has not 
arisen here, the importance of the exogenous variables in the dynamic behaviour of the system suggests that it would be possible to conclude that instrument instability exists in the system when in fact the system does not reveal any underlying tendency for the instruments to explode over the time period of concern. Even in the case where all endogenous variables are weighted and positive costs are allocated to all instruments, it would be easy to conclude through simulation studies that instrument instability does exist if the movement in exogenous variables is severe over the time span of concern, when in fact pure instrument instability of the type discussed by Turnovsky cannot exist. 23 This could lead to an unnecessary increased weighting of an instrument.

Returning now to the original internal and external balance framework of this chapter, it is reasonable to conclude that aiming for either target on an individual basis will cause distruptions to the rest of the system and in particular aiming for an FR target will result in a trade-off with $Y$ and similarly aiming for $Y$ will produce a trade-off with FR. It is clear that we can do better by aiming for both targets, even with one instrument restricted. Experiments IEB5 to IEBI0 are of course extreme examples of exact fine tuning and should only be treated as polar cases as it is fairly obvious that a government will not continuously focus on a balance of payments target at the expense of the income sector. The experiments are useful for placing outer limits on the trade-off between internal and external balance and indicating how single target instrument instability can be disguised or mistakenly indicated when time variant targets and time variant exogenous variables are present in the system. 
DETERMINISTIC CONTROLS IN A STOCHASTIC WORLD

If optimal control or fixed target approaches to macroeconomic stabilisation are to be used in real world applications then the problems of unforeseen shocks to the system will become important. Carrying out monte carlo experiments will not give us the desired result for a particular cost function specification because we could never know beforehand what the actual disturbances will be. In the linear/quadratic framework the control laws are self-adjusting to additive perturbations but for practical purposes this will not be sufficient as we have argued in Chapter Pwo, because for a variety of reasons, government will not be able to react instantaneously (at least in a quarterly model) to the past periods' disturbances except perhaps in the case of open market operations by the central bank. If control techniques are only to be used for historical evaluation and as part of the learning process in gaining additional information about the behaviour of the system and the reaction to possible policy combinations, then it is feasible to simulate the system. with stochastic disturbances. The use of control techniques for future planning will not be able to account for future stocks or adjust in time to account for them when they occur. The preceding analysis has shown quite clearly that while additive disturbances do not necessarily result in a change in the trade-off between targets, significant adjustment can occur in the instrument mix if instruments are in fact able to adjust in accordance with past shocks. This suggests that a knowledge of the size and direction of past shocks would be of crucial importance in actual stabilisation situations (of course policy planners cannot be sure of current shocks, particularly in the income sector) and if some attempt is not made to incorporate them into the stabilisation plan, serious sub-optimal behaviour could occur. 24 To gain some insight into this 
behaviour, the first four experiments were re-computed in a manner which firstly calculated the optimal controls in a perfect information framework and then implemented the controls in a stochastic environment. The values of the welfare function for the particular target and instrument weightings were calculated with the welfare cost indicating the amount of desired path deviation when deterministic costs are implemented in a stochastic world. Two distinct sets of experiments were carried out. Firstly, the average welfare cost was computed from monte carlo replications and secondly a specific example was computed to illustrate some of the economic implications. The illustrative example employed the same shocks as those used in the internal and external balance experiments to enable a meaningful comparison to be carried out. The experiments are identified as IEBI1, IEB12, IEB13 and IEB14 and correspond to the first four internal and external balance experiments. The general results are presented in the following table. The welfare cost in the monte carlo case was computed in the usual manner by averaging out the welfare costs of all replications. Selected results are graphed in Figure 17.

Table 10

IEBII - IEB14. Welfare Comparison

\begin{tabular}{lccr} 
& D & Illust. & $\begin{array}{c}\text { Monte } \\
\text { Carlo }\end{array}$ \\
\cline { 2 - 4 } IEB11 & .223 & 17.5 & 15 \\
IEB12 & 0 & 1131.8 & 960 \\
IEB13 & 78.4 & 1059.6 & 900 \\
IEB14 & 195.7 & 1514.1 & 1070 \\
\hline
\end{tabular}

Note that "D" refers to the deterministic or certainty solution to IEBI, IEB2, IEB3 and IEB4 and that, the welfare cost for the final three experiments is greater than the first due to the difficult scaling of weights. 


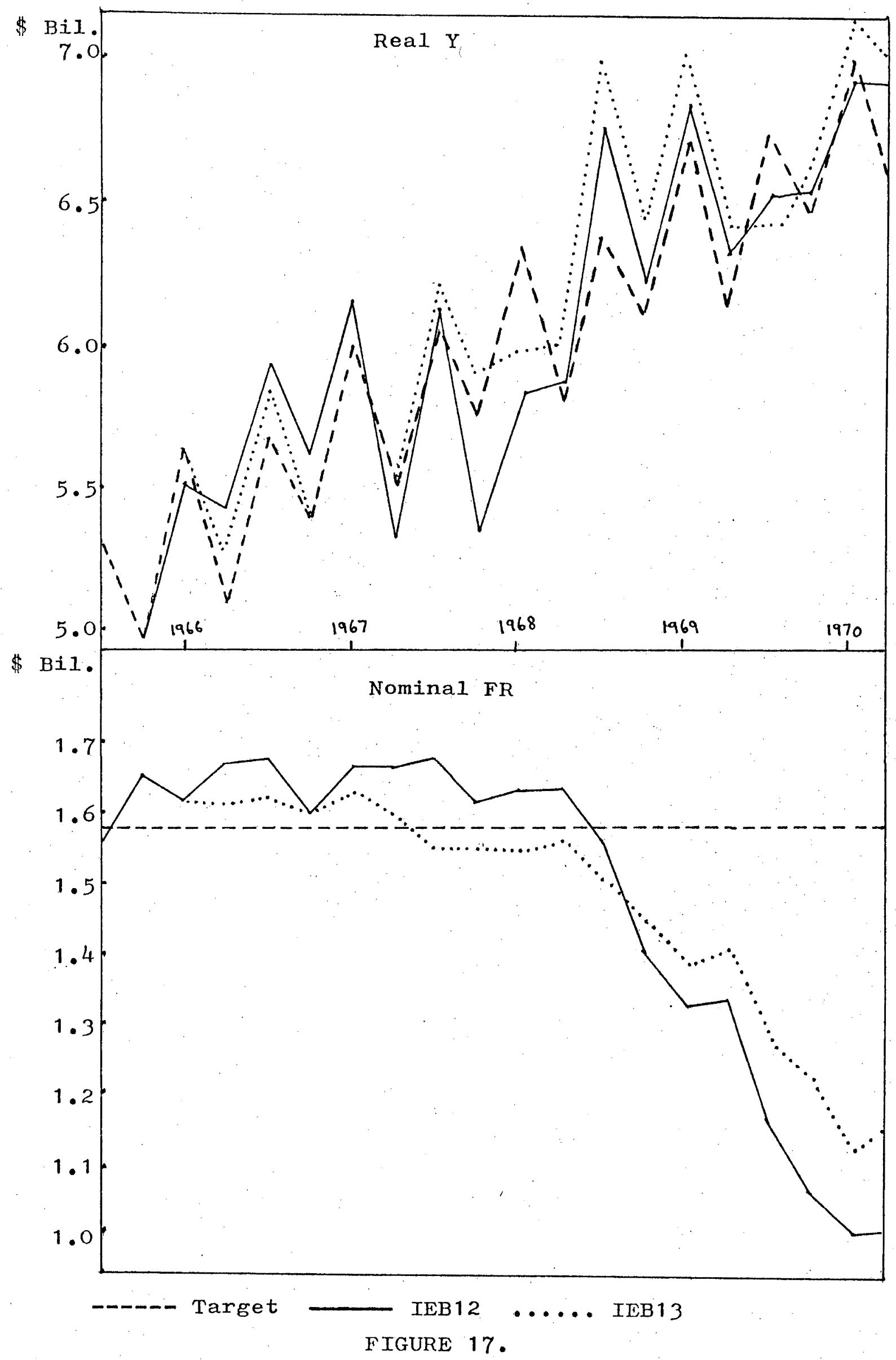


The monte carlo averages lie below the illusxrative costs due to the fact that the same set of weights was employed in each example with those weights producing a solution lying closer to the upper bound replication than to the average. Nonetheless the same general results hold. The increase in the size of the value of the welfare function from deterministic to stochastic should be viewed with caution and not taken too literally or as a general rule as the result will depend on the size of the weights used. Smaller weights will give a smaller increase and vice versa.

The most striking result which can be obtained from inspecting the values in Table 10 is that the implementation of controls developed under the assumption of certainty (whether by choice or necessity) in a stochastic world is potentially disastrous from a stabilisation point of view. All experiments indicate a very significant increase in welfare loss but perhaps the most surprising result is that the case of two targets, two instruments which results in exact deterministic target achievements and near exact stochastic achievement when the controls are allowed to adjust instantaneously to past shocks, is not as acceptable in cost terms as IEB13 when the fiscal instrument is fixed. That is, we can do better by aiming for two targets with only one active instrument than allowing both instruments to be active when past shocks are not incorporated into the solution. The reason for this can be seen from the results of IEB2 where an extensive monetary policy was required in the deterministic case and on even more severe monetary policy was required in the stochastic solution, indicating the high degree of importance attached to being able to adequately compensate for disturbances.

The main cause of the increase in welfare exhibited in Table 10 is the behaviour of FR which, as we have seen, has a tendency to move rapidly off target if additive disturbances are not corrected for. On 
the other hand, $\mathrm{Y}$ is reasonably stabilised in most cases and is a direct result of shocks to the income sector being of considerably smaller relative magnitude than shocks to the open and monetary sectors. The size of the shocks is of course a direct result of the structural estimation where the income sector provides "a better fit" to the data than the open sector. This situation is not too far removed from the real world as it is likely that the open sector would be subject to greater unforeseen shocks than the income sector due to the inability of planners and forecasters to adequately predict what will happen in the world economy and how the world economy will affect Australia.

The results also suggest that a quarterly model may not be sufficient for planning purposes when the foreign sector is incorporated in the model. Reasons for this have been suggested in Chapter Three and further elaboration and evidence is provided in Chapter Eight. The major objection to a quarterly model is that it does not allow monetary policy to operate in the very short run such as monthly or weekly which is the case with actual monetary policy (open market operations) in Australia (and other countries for that matter). The result is that cumulative shocks could occur. It is also likely that the monetary authorities will have sufficient information about shocks contained in the previous quarters' reserves to allow instantaneous adjustment of the monetary instrument for the current quarter, but not so for the income sector and fiscal policy which is subject to a wide variety of lags and implementation problems which require that future levels of spending be determined some time before that level is manifested in the system. The experiments reveal in a graphic way what could occur if the authorities are forced to plan in advance of the planning period and are therefore unable to fully take account of any shocks. Even stochastic 
forecasts of the future will not be adequate as we can only attempt to predict "average" shocks (which of course will converge to zero) and effects when in fact single shocks occur in any given time period. Once again, the analysis would perhaps suggest that optimal control would be better confined to historical evaluation and insight about possible policy combinations, rather than actual planning due to the effects of uncertainty. The results presented above can only be regarded as an extreme example as different instruments will be capable of instantly adjusting to past shocks such as S.R.D. ratios and tax rates but only if the correct information is forthcoming about the value of the lagged state vector, $x_{t-1} \cdot 25$ Further information about the behaviour of the system under deterministic control in an uncertain world will be provided by experiments in following chapters.

\section{HISTORICAL EVALUATION OF OPTIMAL ECONOMIC POLICIES}

Assuming that the model adequately reflects the basic instrument responses of the Australian economy then it is fairly clear from the results that the government could have done better in relation to the targets of internal and external balance by allowing the monetary instrument to be more active. The results of IEBI indicate that the two targets could be stabilised more effectively than the historical performance, even with restricted instruments, when a set of structural coefficients are available to the planner. Of course, the government of the day may have had a different set of preferences in mind and may have been more concerned with stabilising $Y, F R$ and additional targets such as $M$ and $C$. The historical time path of DM indicates, as we have said, that the authorities appeared to be cautious in the application of the monetary instrument. The fact that historically, the monetary instrument was not used in a stop-go manner as severely as the optimal monetary policy in IEB1, IEB2 and IEB3 
(see Figure 12) doesn't necessarily rule out an historical cost function specification of only $\mathrm{Y}$ and FR but could just reflect a lack of information and it should be recalled that the preceding experiments assumed full information about the structure, the uncontrollable exogenous variables and in the stochastic case, certainty about the distribution of the errors, even if the exact value of the stocks were not known until after they occurred.

To gain a depper insight into the historical performance of stabilisation policy it would be beneficial to know the specification of the governments cost function, if indeed it had one, over the period of concern. Theoretically this is a simple matter. We began with a specific set of preferences, a model whose structure was known with certainty and certainty about the exogenous variables. From this information linear feedback equations were obtained. It should be clear that we can work backwards starting with the feedback equations and ultimately determining the initial preferences. However, this procedure would break down in a real world analysis as it would depend on having the absolutely correct model specification and estimation which exactly characterised the economy and furthermore, was known with certainty by the planning authorities of the time. Several attempts have been made to carry out this type of analysis beginning with the early work of wood (1967) and more recently Friedlaender (1973). The results are unconvincing, for example, the work by Friedlaender abstracts from the large number of exogenous variables that are present in the system and assumes that the governments preferences were quadratic, and that the Wharton model is an exact replica of the economy. Even the abstraction from exogenous variables in the system will make the estimation of historical feedback equations untenable. This is clear from the preceding analysis. The feedback 
matrices presented in Tables 6 and 7 show that the optimal control in any period depended substantially on the past behaviour of the system and upon the newly defined auxiliary variables of the state space realisation. What they do not show is that the additive vector, $\mathrm{f}_{t}$ ' is equally as important and this vector varies substantially from period to period as it is largely a function of the exogenous variables. Table 11 indicates the degree of divergence of $f_{t}$ per time period for experiments IEB1, IEB2, IEB3 and IEB4.

Table 11

\begin{tabular}{|c|c|c|c|c|}
\hline & & $\begin{array}{l}\text { Additive } \\
\text { Period } \\
1 \\
\end{array}$ & $\begin{array}{c}f_{t}{ }^{\prime}- \\
\text { Period } \\
10 \\
\end{array}$ & $\begin{array}{l}\text { period } \\
\text { Period } \\
20\end{array}$ \\
\hline \multirow{2}{*}{ IEB. 1} & $\mathrm{G}^{\prime}$ & 1290 & 1456 & 2180 \\
\hline & DM & 970 & 1264 & 476 \\
\hline \multirow{2}{*}{ IEB 2} & G & 1427 & 1592 & 1885 \\
\hline & DM & -3956 & -4283 & -2975 \\
\hline \multirow{2}{*}{ IEB3 } & G & 1110 & 1158 & 1527 \\
\hline & $\mathrm{DM}$ & -3553 & -3930 & -2957 \\
\hline \multirow{2}{*}{ IEB 4} & G & 110 & -113 & 1819 \\
\hline & DM & 891 & 1110 & 1472 \\
\hline
\end{tabular}

How then in a least squares estimation can the correct intercept term be found? The relevant feedback matrices also show how the coefficients of particular lagged state variables can differ considerably over time. For example the coefficient relating $G$ to lagged $F R$ for periods one, ten and twenty in IEB4. A least squares estimation will yield only one coefficient for a particular variable. Chow (1972a) has also attempted a crude historical analysis of policy by working in reverse to the formation of optimal policy by initially estimatịng historical feedback matrices, using the lagged variables of his model as the explanatory variables. The 
analysis cannot be regarded as a significant contribution as the model contains no exogenous variables whereas the simplicity of the model suggests that important areas have been omitted which could have been treated as exogenous and the amount of endogenous feedback is limited. The net result is that the least squares estimated intercept, $f_{t^{\prime}}$ and the optimally derived intercept do not tally as could be expected with a further result being that the feedback coefficients cannot possibly be correct. Furthermore, the least squares version of the historical feedback matrices only explain approximately two percent of the variation in monetary and fiscal policy and while the coefficient of determination should not be considered the beall and end-all of the acceptability of an equation, it does suggest that among other things, either Chow's model is substantially incorrect or that the government did not have a set of quadratic preferences or the same targets as chow (assuming that the model is correct). In either case the results are highly dubious and substantially inferior to Friedlaender's approach. The exclusion of relevant exogenous variables, however, will ensure that the "true" intercept cannot be obtained unless of course the government used the identical model to that proposed by chow to carry out stabilisation policy - a highly unlikely occurrence in view of the exceptionally crude nature of the model. As a further example of the futility of attempting to work in reverse to obtain historical preferences, a similar exercise to Friedlaender's (although Friedlaender did not include the auxiliary variables which are important - see Tables 6 and 7) and Chow's was carried out using the model employed in this study. The results, as could be expected, are worthless due to the multicollinearity between the large number of lagged variables which appear as explanatory variables in the feedback equations for $G$ and DM and for the reasons. outlined above. The results have there fore not been included. An attempt 
to remove the multicollinearity by deleting variables or aggregating variables did not rectify the problem and even if it did, the aggregation and deletion of variables would ensure that the true feedback equations would not be obtained and even if the model was the unique, exact, true replica of the economy, the incorrect feedback matrices would result in incorrect preferences being obtained.

What value then is an attempt to analyse the historical behaviour of stabilisation policy? The attempts to systematically ascertain past policy and target preferences are extremely limited given the existing state of knowledge. The argument outlined above only touches the surface of the problem but serves to indicate the limitations. The best method in the absence of a knowledge of historical preferences is probably to simply compare optimal and historical results, and assuming that the model adequately represents the trade-offs and policy impacts in the system (although the exact structure of the economy may differ from the model), then evaluate the relevant policies in light of the similarities between the general behaviour of historical and computedsituations. To reiterate, using this technique it would be reasonable to conclude that the Australian government did not specifically aim for internal and external balance alone, or if they did they were largely unsuccessful due to the lack of adequate adjustment in the monetary instrument in particular (see Figure 12 for historical DM). Note that comparisons are carried out in terms of deterministic results as presumably historical shocks would be built into the data used to estimate the structural coefficients. $Y$ has not been specifically analysed as it was reasonably well stabilised historically and effectively stabilised in the first four experiments of this chapter.

The results obtained in this chapter show that the simultaneous achievement of internal and external balance under a fixed exchange rate 
regime can only be exactly achieved in the deterministic case and then only if both monetary and fiscal policy are allowed to adjust freely. Even in the presence of additive disturbances, internal and external balance can be almost exactly achieved but only by using both instruments in a rigorous fashion. The results also indicate that the monetary instrument should only be directed towards external balance (rather than internal balance) which is an example of Mundell's (1962) Principle of Effective Market Classification and which concurs with the theoretical results obtained by Turnovsky (forthcoming). In terms of exact fine tuning, it can be seen that the system can be effectively stabilised with one instrument or effectively stabilised with many targets and the instruments weighted equally with the targets, and the closing of the usually small gap between acceptable time paths for $\mathrm{Y}$ and FR and the respective targets required a considerable shift in the use of the instruments, particularly monetary policy. Pindyck (1973a) has shown how it may be necessary in a closed economy for the optimal monetary policy to be a policy which consists of strong bursts. A similar conclusion holds for the open case when the open sector target of external balance is added to the closed target of a specified level of income. The results show that monetary policy, in contrast to fiscal policy, is used in strong expansions and contractions in a stop-go manner, except when monetary policy is explicitly prevented from deviating from its target path.

The introduction of additive disturbances prevents exact fine tuning and results in a substantial welfare loss which concurs with the results of Chow (1972a), Pindyck and Roberts (1974) and Garbade (1975a) for example. In addition, the introduction of additive disturbances resulted in the optimal mix of policies changing relative to the deterministic results with the change in some cases being quite dramatic, 
particularly in monetary policy, although it must be conceded that in many cases (not all) the characteristics or general behaviour of the optimal stochastic policies do not differ greatly from the deterministic results. The emphasis on different instruments can change, for example, the mix of policy from a strong use of both policies to a more extensive use of monetary policy and a less vigorous fiscal policy in the stochastic case. Turnovsky (1975) has shown how the introduction of parameter uncertainty can lead to a switch in the choice of optimal instrument. Although Turnovsky's theoretical work refers to the optimal choice of monetary instrument, it is still possible to make a general comparison. The experiments reported in this chapter illustrate how the presence of additive disturbances can switch the emphasis placed on particular instruments. Of course, the problem does not allow the complete exclusion of one instrument as can be done in Turnovsky's framework but nevertheless there are clear switches in preference between the extensive use of a particular instrument relative to another. In this general aspect, the results provide some empirical support for the theoretical work without resorting to parameter uncertainty.

The problems associated with achieving internal and external balance in either a deterministic or stochastic framework under a fixed exchange regime, are highlighted by the trade-off between the monetary instrument, foreign reserves, capital flows and the total monetary base. Any attempt to neutralise the effect of foreign reserves on the domestic economy necessitates strong monetary action which produces instability in the monetary sector. Under these circumstances, external balance may not be a desirable target. The instability in the monetary sector raises the possibility of incorporating a monetary target, for example the rate of interest, as a major target in the cost functions of policy planners. 
Experiments which feature the incorporation of monetary targets in the cost function along with $\mathrm{Y}$ and $\mathrm{FR}$ are reported in the chapter on the fixed target approach to stabilisation.

The results presented in this chapter have a broader application than merely to the problem of achieving internal and external balance. The results illustrate quite graphically the problems that can arise from imperfect information. The co-ordination and control of economic activities becomes extremely difficult under uncertainty. This is especially so when the presence of uncertainty gives rise to different and sometimes opposite policy applications to those required under certainty. If policy is formulated, whether optimal or non-optimal, on the assumption of full information, then incorrect policies can easily be applied with disastrous results. 


\section{OPTIMAL ACHIEVEMENT OF MONETARY TARGETS}

A common approach to the theory of monetary policy is to treat the money supply as an instrument which the central bank can directly manipulate in the same manner as government spending. Similarly, in many instances it is convenient to treat the rate of interest as an instrument variable. The treatment of the money supply and the rate of interest as instruments has not been solely confined to the theoretical literature. Applied econometric studies have for a long time treated the money supply, in particular, as a control or exogenous variable. (There are of course examples of studies treating both variables as being endogenous but these are in the main restricted to the few very large models that have been constructed.) The treatment of the money supply and the rate of interest as controls is of course a simplified abstraction, even in a deterministic world. Both variables are in fact endogenous and are simultaneously determined by the interaction of other endogenous variables in the system and the genuine monetary and fiscal instruments available to the authorities. In this respect a more appropriate view to adopt in relation to the money supply and the rate of interest would be to treat both variables as proximate targets through which the achievement of what we may term ultimate targets, (income, unemployment, inflation and so on) can be attained by stabilising our proximate targets in accordance with some specified set of target values. Implicit in this approach is the belief that there is some unique link between proximate targets and the ultimate targets in the system. Even in a deterministic world, the simultaneous link between the money supply and the rate of interest may be so significant that the monetary authorities are prevented from achieving 
targets for both, unless both targets are perfectly consistent. In the case of incompatible monetary targets it would be necessary to trade-off targets or drop the desire to hit both targets and aim for either one. If the economy is closed and the only ultimate target is, for example, real income, it could quite reasonably be concluded that either proximate target would be appropriate. When the system is opened up the choice of proximate target becomes complicated by the reaction of the open sector to the achievement of a particular target and more importantly by the feedback of the open sector into the system as a whole, particularly the monetary sector. This may exclude both the money supply and the rate of interest as proximate targets to be directly aimed for.

In this chapter, the choice of proximate target will be studied in the framework of the model developed in Chapter 3. In particular, the trade-offs between $M, R L$ and $M B$ will be studied along with the effects of hitting a monetary target on the ultimate objectives of $Y$ and FR. The results of the preceding chapter suggested that if policy planners wish to maintain internal and external balance then some instability in the money supply and the rates of interest must be tolerated. A further aim of this chapter will be to determine whether or not the reverse is also true. That is, will the achievement of a proximate target produce instability in the internal and external sectors? If instability does result then serious doubt must be placed on utilizing the concept of proximate targets. The use of what may be termed "true" controls to achieve a proximate monetary target should not be confused with the monetary instrument problem, although in some respects they are closely related (Waud (1973)), which first appeared in Pooles' (1970) seminar paper and which has been the subject of a profuse literature since. See for example Moore (1972), Sargent (1971), Turnovsky (1975) and Kareken (1970). It should be noted 
that the instrument problem as defined in Poole and subsequent papers, only becomes non-trivial in a stochastic theoretic framework. The proximate monetary target problem as presented here can be non-trivial in a deterministic world depending on the behaviour of the remainder of the system, particularly the behaviour of the ultimate targets and the degree of adjustment required by the controls. The results that follow support this assertion.

The work of Pindyck and Roberts (1974) (1976) (PR) has probably been the predominant applied study of recent times in relation to studying the effects of hitting a money supply or interest rate target in an optimising framework. This is not to say that $\mathrm{PR}$ have produced the only work in this area, for example Friedman (1975a) and Garbade (1975a) have also addressed monetary problems via an optimising framework, but the work of PR has probably been the most specific and has appeared in journals which has ensured a wide readership by control theorists. For these reasons, the results presented in this chapter are specifically compared with the PR results. Before proceeding to a discussion of the experiments it is worth noting that the PR studies suffer from treating the monetary sector in isolation with the consequence the $\mathrm{Y}$ is treated as exogenous. ${ }^{26}$ Placing the monetary sector into a fully integrated model would mean that every adjustment to $M$ or $R L$ would produce a response in $Y$ which would in turn produce a further adjustment in the proximate targets. The model used in this study is a modest attempt to incorporate feedback from sector to sector and as we shall see in the experiments that follow, the presence of such a feedback results in fiscal policy being able to be manipulated in response to monetary targets as well as income variables, a feature which is absent from PR's analysis. A model which incorporates both monetary and income sectors in a close interrelationship highlights the problems of 
decentralised policy making when any action by fiscal authorities who may be aiming exclusively for an income target, results in monetary adjustments which can shift the money supply, for example, off the monetary authorities target path and vice versa.

One further point needs to be discussed and that is if we have a complete structural system, why bother to aim for proximate targets in the first place? The question is valid but it is a fact of life that monetary authorities persist in adhering to monetary targets, particularly the money supply, regardless of the compatability of such a target with any specified time path for $\mathrm{Y}$, for example. By focusing solely on the achievement of a monetary target information can be obtained firstly about any trade-offs between monetary variables and secondly information can be gained about trade-offs between monetary variables and the remainder of the system.

The experiments that follow focus on the achievement of one particular proximate monetary target. The targets for $M, R L$, RS and MB are those which are specified in Chapter 4 and once again a twenty period time horizon is used. The retention of the exact experimental structure to that employed in the internal and external balance experiments ensures that valid comparisons between experiments can be made. The experiments that follow are identified by the letter $M$ and a numeral designating the number of each monetary experiment.

THE OPTIMAL ACHIEVEMENT OF THE MONEY SUPPLY TARGET

The first experiment (MI) consisted of forcing the money supply. to follow its target and at the same time emphasising monetary policy relative to fiscal policy with the relative weighting in the cost function being - 


$\begin{array}{ccc}M & \text { G } & \text { DM } \\ 2000 & 500 & 1\end{array}$

Following Chapter 4, weights were adjusted each time period to avoid cost function bias and to ensure that the above relativity was maintained for the entire planning period. Even though the weight on $\mathrm{G}$ is high, with the weight on $M$ being four times that on $G$ we would expect some adjustment in G to complement DM.

The optimal results indicate that the achievement of a steady growth in the supply of money is feasible, although the money supply did not hit its target until approximately half way through the planning period. One would expect that under conditions of certainty and instantaneous policy response (within the unit time period), monetary policy could be manipulated to keep $M$ on target. It must be concluded that the relatively greater restriction placed on fiscal policy has been the influencing factor with the adjustment in $\mathrm{G}$ being less than expected. If fiscal policy was allowed to adjust freely then some of the emphasis could be removed from the use of monetary policy, suggesting that fiscal policy needs to be specifically directed towards the money supply target (allocated zero costs in the cost function).

In relation to the target for DM, monetary policy has been used extensively and severely throughout the planning period. The optimal path is characterised by huge expansions and contractions. It is doubtful if the private sector could be persuaded to shift its portfolio composition in such a drastic manner in such a short time, thus throwing some doubt on the feasibility of this action. The severe monetary policy required to achieve the money supply target has important consequences for the remainder of the monetary and open sector variables. Severe upward and 
downward pressures on the term structure of interest rates has occurred. The short rate drops to $2.44 \%$ in period sixteen and then climbs to $10.0 \%$ four periods later. The close relationship between the long rate and the short rate means that the optimal path of the long rate will closely follow that of the short (the behavioural pattern not the values). This does occur with the long rate falling to $3.0 \%$ in period sixteen. Figure 19 indicates the severity of the movement in the long rate. The behaviour of the rates of interest indicate that given the relative importance attached to monetary policy, fiscal policy and the money supply, the pursuit of a money supply target will induce instability into asset markets. The model is not sophisticated enough to capture the full effects of asset market instability but it seems reasonable to suggest that such instability in interest rates could have far reaching effects on the financial sector, particularly through changes in expectations and possible loss of confidence experienced by financiers and investors. If orderly asset markets are deemed to be highly desirable then an interest rate proximate target may be more appropriate.

So far we have ignored the consequences of pegging the money supply on the ultimate targets such as $\mathrm{Y}$ and FR. The optimal path for real $\mathrm{Y}$ is stable and feasible as it tracks reasonably close to its target (Figure 18) with the exception of the final time periods in which monetary policy is restrictive. However, the divergence is not severe as $G$ is close to its target and is of an expansionary nature which tends to partially offset monetary policy. In contrast to $Y, F R$, or the external balance position, is very unstable. This is a result of the severe stop-go optimal monetary policy which in turn results in huge variations in government generated domestic liquidity. As a result of the portfolio equilibrium nature of capital flows, substantial compensating movements 


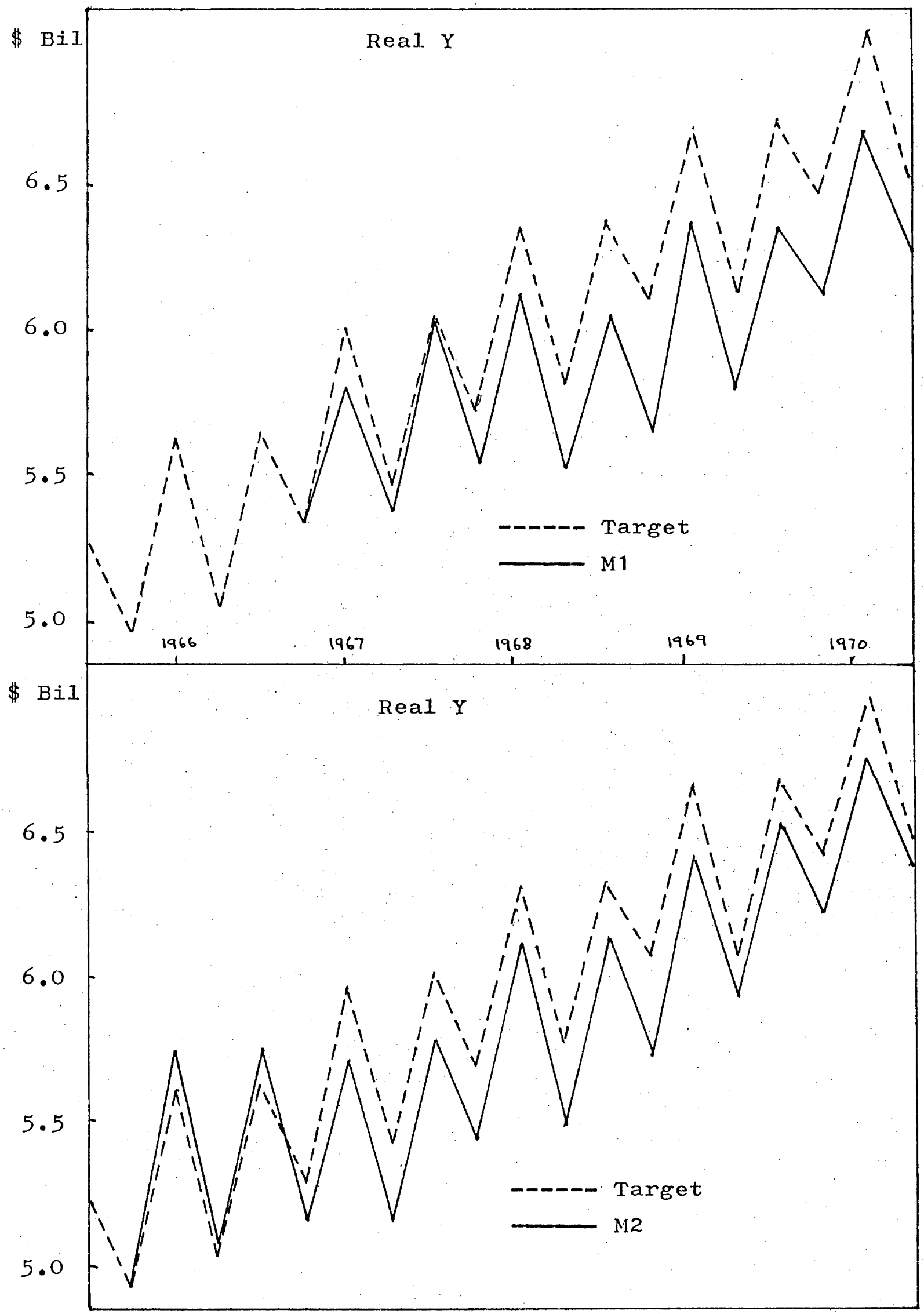

Figure 18. 


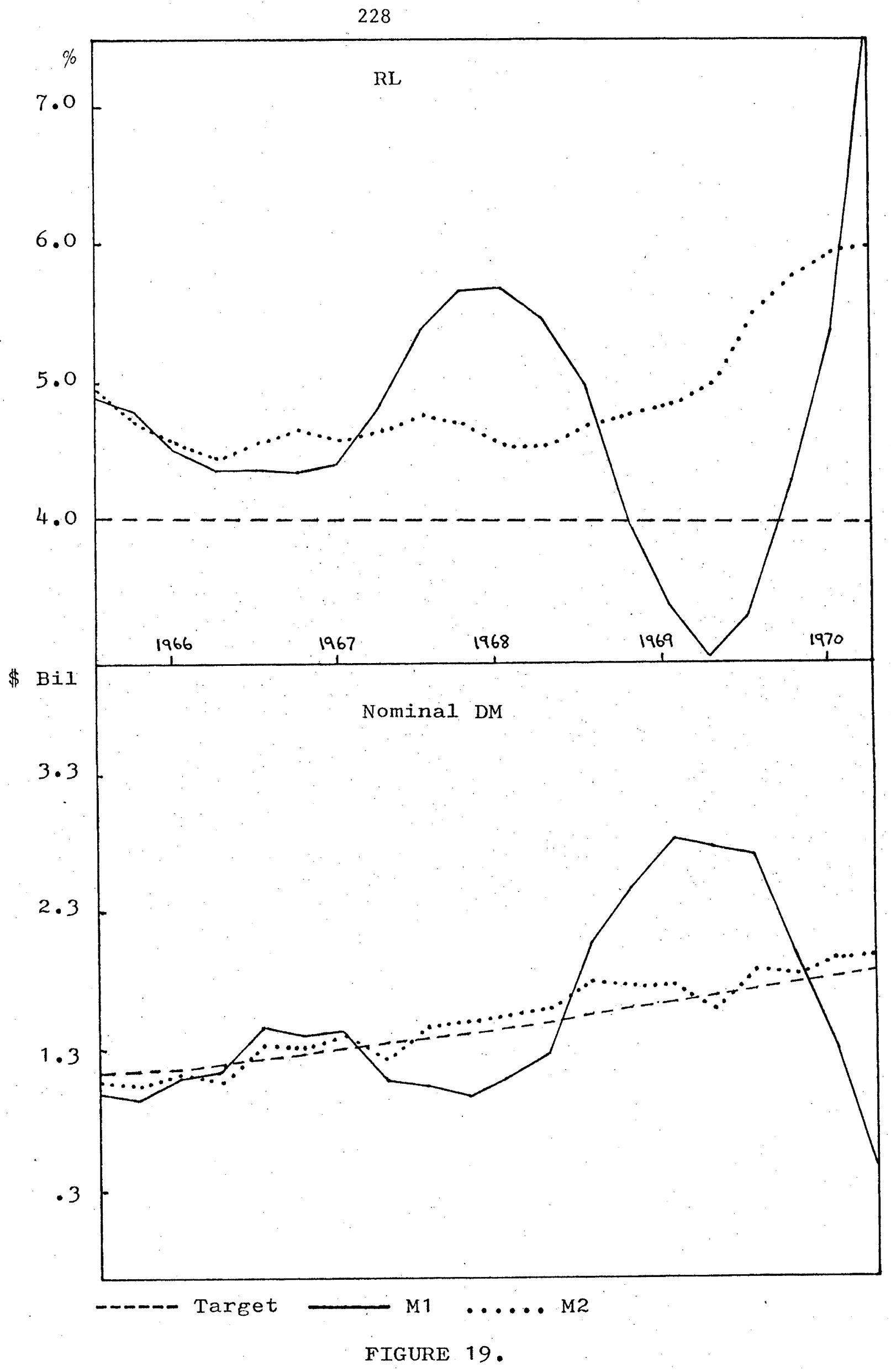


in capital inflow are induced, creating a similar movement in foreign reserves. Note that while monetary policy upsets the external sector, the external sector feedback into the monetary sector induces even more instability in monetary policy as the monetary instrument must then be used to offset this feedback. The instability generated in the interest rates and the open sector seems to indicate that if the authorities wish to hit a money supply target then the reliance on monetary policy to the exclusion of fiscal policy, which intuitively seems to be a reasonable course of action to adopt, is not a particularly satisfactory mix of policy. Fiscal policy is also required.

The second experiment (M2) consisted of once again allocating very high costs to deviations of the money supply from its target but at the same time fiscal policy was weighted equally with monetary policy to try and remove the instability found in experiment one. The relative weights of the variables were as follows -

$\begin{array}{ccc}M & \text { G } & \text { DM } \\ 2000 & 1 & 1\end{array}$

The money supply is exactly on target from the beginning of the planning period to the end. The relaxation of the restriction on $G$ has resulted in a significant shift in the optimal mix of policy from that obtained in experiment one and confirms the earlier assertion that fiscal policy is required in addition to monetary policy to achieve a money supply target in an open economy. The stop-go, excessive and probably unobtainable optimal monetary policy of the previous experiment has been replaced by a relatively smooth expansion of base money, only occasionally interrupted by a more severe expansionay movement whereupon the optimal path returns to a steady expansion almost immediately after. The nature of the optimal monetary policy implies a more restrained intervention by the central 
bank into the asset market and greater stability in the composition of the private sector's portfolios.

The implementation of a more subdued monetary policy has meant that a more extensive and expansionary fiscal policy to that obtained in Ml has been employed (see Figure 20). Recall that in experiment one $G$ was largely on target. In some respects the broad behavioural pattern of $G$ resembles the behaviour of DM in the first experiment, thus in effect, combining with a stable monetary policy to produce a reversal in the application of both instruments. The change in the policy mix has not substantially altered the optimal path for real $Y$. The strong bursts in fiscal policy required to keep $M$ on target, combined with the steady monetary policy, have the effect of keeping $Y$ close to its desired time path. This is a particularly encouraging result as it allows the planning authorities to select a mix of policy which is as complementary to the income sector as it is to the monetary sector. The fact that a 1.58 quarterly increase in the money supply is consistent with policy stability and a time path for real $\mathrm{Y}$ which tracks close to its desired path (see Figure 18) supports the contention that under certain circumstances it would appear to be feasible to focus on a proximate target. The acceptability of such a situation depends on the behaviour of the rest of the system.

A close inspection of the structural equations reveals that there is a trade-off between $D M, F R, C F$ and $M B$ which has a direct impact on the money supply. If the money base and hence money supply are forced to follow a smooth path then substantial adjustment would need to take place in the external sector. In experiment one, FR carried the burden of adjustment over the other non-control and non-proximate target variables. The results of this experiment reveal that the behaviour of 


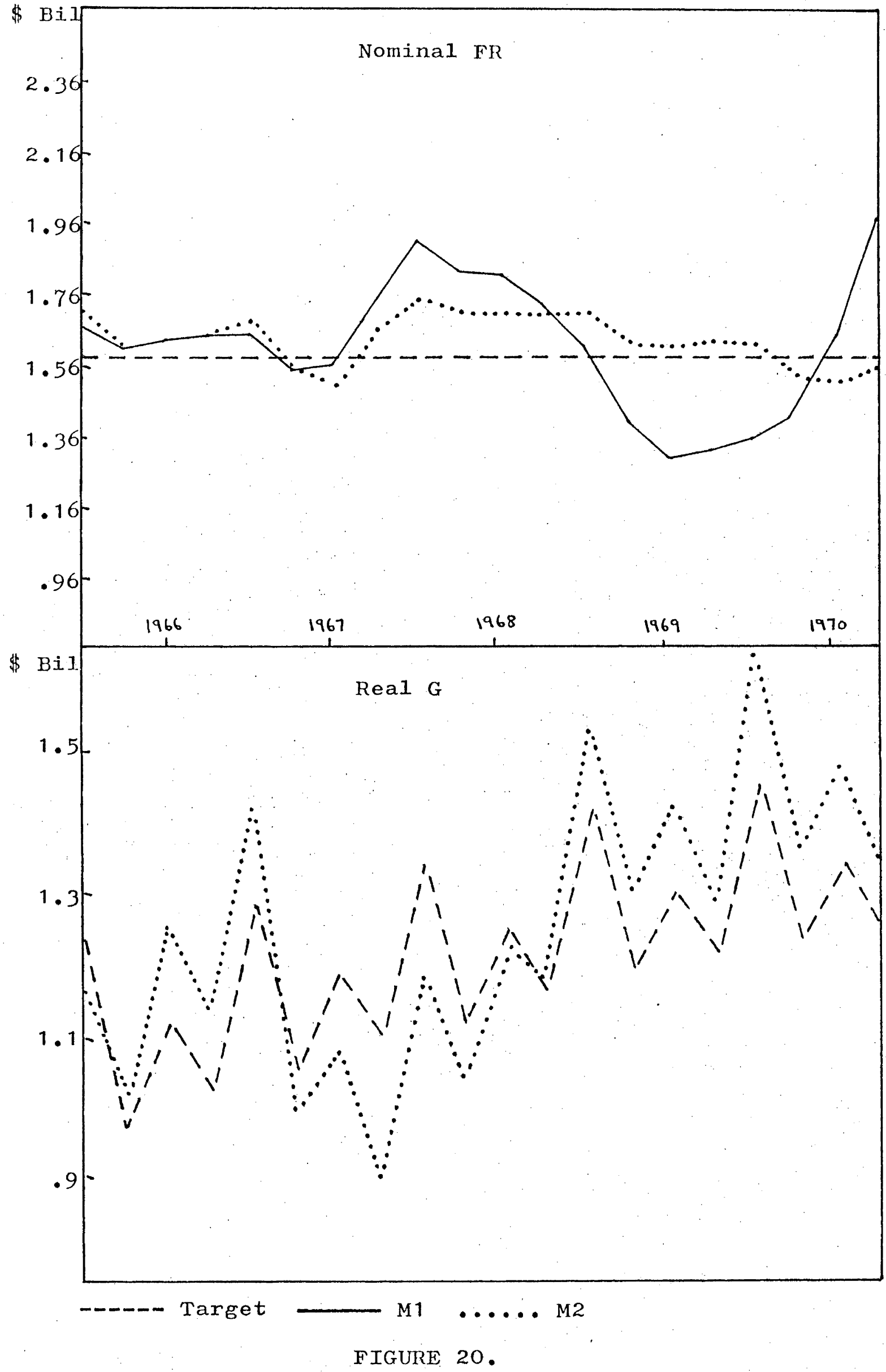


FR has been improved (along with DM) which of course is a direct result of the more subdued monetary policy. Fiscal policy generates a greater degree of fluctuation in the current balance than in the previous experiment but the influence of monetary policy in the capital flows equation ensures that capital flows do not fluctuate erratically as before and contributes to the increased stabilisation of the open sector. As before, the feedback from the open sector to the monetary sector is important but in this case it is complementary to monetary policy in the sense that severe monetary policy is not required. The other area of importance is the behaviour of the interest rates. Figure 19 reveals that the severe cyclical movement which was characteristic of the first experiment has been eliminated. After an initial decline the otpimal path settles down to a continued expansion; a trend which may need to be reversed in the future to overcome any detrimental effects on investment and income. This again raises the question of whether or not an interest rate target would be more appropriate.

The results presented so far indicate that an appropriate weighting of monetary and fiscal policy can produce a mix of policy which will keep the money supply increasing at a constant rate and which is consistent with a stable optimal path for $\mathrm{Y}$, traditionally regarded as the major ultimate target. We can also obtain an acceptable set of time paths for interest rates and external balance although it must be pointed out that a trade-off between $M, R S$ and hence $R L$ does occur as was suggested in the introduction, a trade-off which can become severe with an inappropriate policy weighting. The results also indicate that we can follow a Friedman type rule although we are operating in a non-Friedman world subject to requiring that both fiscal and monetary policy be directed towards the money supply target. This last requirement rules 
out the possibility of setting government spending in accordance with political and social welfare requirements, that is, treating it as passive in relation to the economic requirements of the system as in experiment $\mathrm{Ml}$. THE OPTIMAL ACHIEVEMENT OF THE INTEREST RATE TARGET

Theoretical work on the choice of proximate target generally consists of choosing the money supply or 'the' rate of interest with only one rate being present in the model. The model presented in this paper contains both a short and long rate linked by a simple term structure thus permitting only one rate to be directly controlled. As the long rate, RL, directly influences income it would seem appropriate to focus on this rate for policy purposes. This procedure will of course break the direct simultaneous link between the money supply and the short rate. The term structure ensures that if we force the long rate to follow its target we will also be imposing a highly restricted time path on the short rate, effectively pegging it and indirectly maintaining any trade-offs which may be present in the model.

The third experiment to be reported (M4) assigns high costs to deviations of RL from its target and zero costs to all other state variables. The relative weightings were as follows -

$\begin{array}{ccc}\text { RL } & \text { G } & \text { DM } \\ 2000 & 1 & 1\end{array}$

The results indicate that $\mathrm{RL}$ was on target throughout the planning period with the achievement of the proximate target being consistent with total instability in the income sector. The instability arises from directing fiscal policy through $\mathrm{CB}, \mathrm{CF}$ and $M$ to achieve the interest rate target. Figure 21 illustrates what must be foregone in terms of national income to achieve the interest rate target. We have in fact a reversal in the 


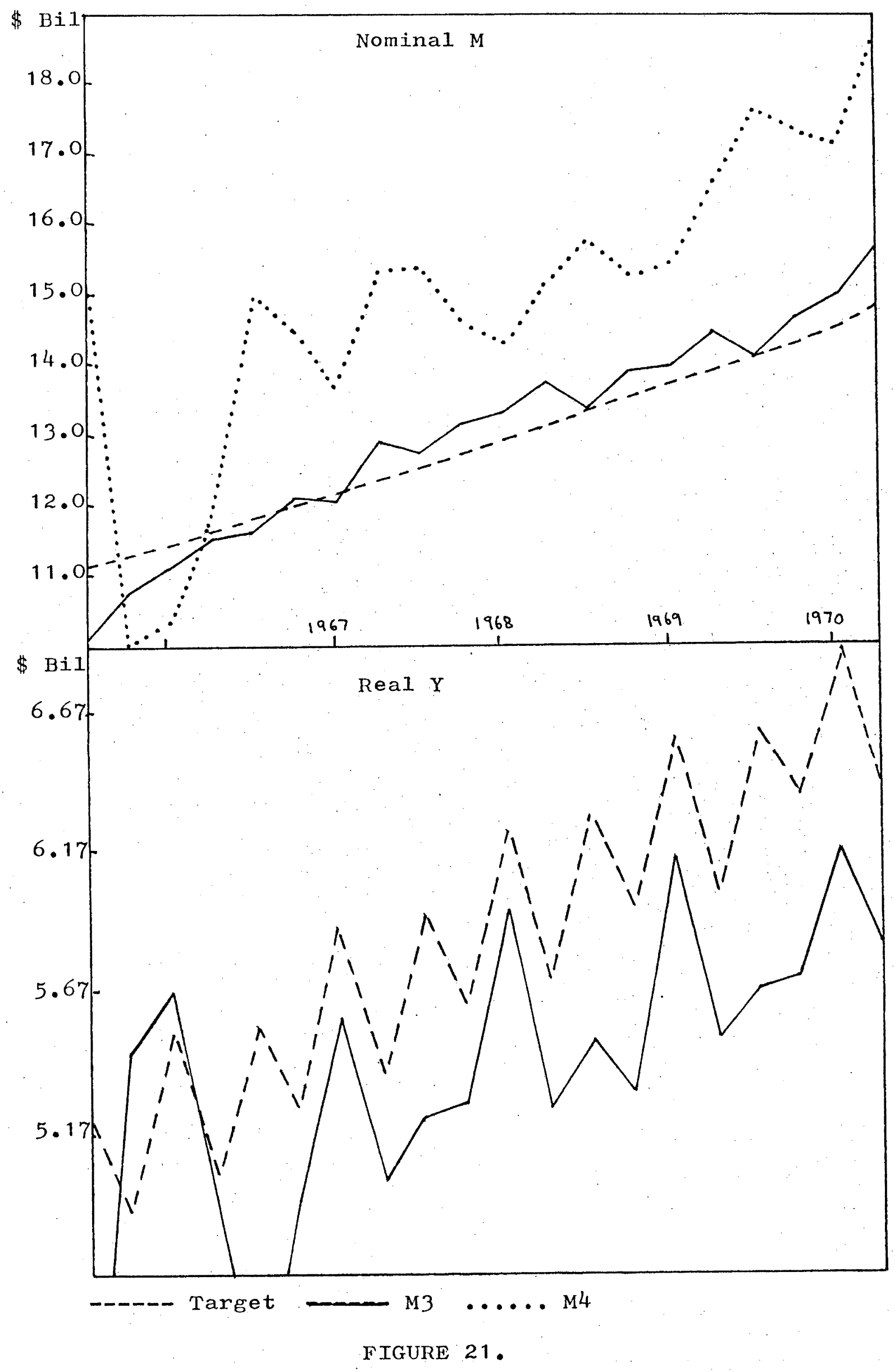


result obtained in $\mathrm{M} 2$ where both instruments were also assigned to the proximate target. Rather than having freely adjusting instruments being consistent with stability as in experiment two, we now have freely adjusting instruments being consistent with instability. The results of the third experiment are indicative of a situation in which the achievement of the interest rate target results in a trade-off between the income sector and the money supply and open sector with the income sector being totally unacceptable. The pegging of the rate of interest has not produced instability in the money supply. In comparison with experiment one this is a more satisfactory result as in the initial experiment the achievement of the money supply target resulted in severe fluctuations in RL. The external balance position, FR, is also acceptable in comparison with the two previous experiments. Of particular importance is the absence of the severe rundown in foreign reserves found in experiment one. The optimal path of foreign reserves begins in a manner which appears to be a forerunner of instability but then becomes almost perfectly stabilised, although at a very high level. Similarly, the optimal monetary policy, after an initial contraction, settles down to move in the general direction of the target suggesting that severe open market operations are largely unnecessary (with the exception of the initial contraction and subsequent expansion). The relaxation in the use of monetary policy is of course a direct result of the excessive and unstable use of fiscal policy, with the subdued monetary policy not generating undesirable feedback responses from the open sector requiring further compensatory and severe monetary policy. The behaviour of $G$ and $Y$ would immediately eliminate the use of the particular policy weighting employed in this experiment but the results do suggest that if policy planners wish to achieve an interest rate target and maintain a stable 
income sector then the remainder of the monetary sector and especially the open sector may need to be sacrificed. The following experiment substantiates this assertion.

One question that arises from this analysis is whether or not it is actually feasible to hold interest rates constant over time in an open growing economy, given a different policy mix from that used above. It may be that there is no optimal $\mathrm{mix}$, or for that matter non-optimal mix, of monetary and fiscal policy which is capable of holding rates constant and stabilising the system at the same time. It is unlikely that a stable rate of interest would be preferred to an unstable income sector. Adherents to monetary policy would argue that monetary policy can be used to keep interest rates down and still maintain system stability. The applicability of this view in practice is, as the results show, conditional upon the complementary use of fiscal policy and the degree of impact of the open sector on the remainder of the system with the significance of the open sector also influencing the use of monetary policy. To gain a more definite picture of the use of monetary policy to peg interest rates we would need to consider the situation in which fiscal policy is purposefully directed away from the interest rate target, that is, fiscal policy is treated as a passive instrument. This procedure was adopted for the fourth experiment.

The fourth experiment consisted of placing high costs on deviations of $\mathrm{RL}$ and $\mathrm{G}$ from their respective targets while leaving DM free to adjust. The relative weighting in the cost function was as follows -

$\begin{array}{ccc}\text { RL } & \text { G } & \text { DM } \\ 2000 & 1000 & 1\end{array}$


The optimal path for RL closely tracks its target with the exception of minor deviations at the beginning of the planning period. The delay in the exact achievement of the target is due partially to the fact that monetary policy only operates on the long rate through the short rate and the importance of the dynamic characteristics embodied in the long rate equation. The delay is also a result of the inability of fiscal policy to adjust as in experiments $\mathrm{M} 2$ and $\mathrm{M} 3$ where the proximate target in question (alternatively the money supply and the long rate) was achieved exactly from the beginning of the planning period. This is an important result, when combined with the results of $\mathrm{M} 2$, as it indicates that in an open economy monetary policy alone is insufficient to achieve monetary targets exactly due to the close two-way relationship between the monetary and open sectors. Assistance from fiscal policy is also required.

An improvement in the performance of the income sector has accompanied the change in emphasis on instruments. The optimal path for real $\mathrm{Y}$ is very close to its target, which once again is not surprising as $G$ is very close to its target (compare with Ml). The burden of adjustment is carried by monetary policy, the optimal path of which is characterised by an initial extreme contraction, producing similar. behaviour in the money supply with the time paths of both variables being more undesirable than the corresponding time paths in the third experiment. In contrast to real $\mathrm{Y}$, the optimal path for foreign reserves exhibits some instability, initially induced by the severity of monetary policy and thereafter compounded by a combination of monetary policy and extremely erratic and totally unacceptable capital flows. The time path of $\mathrm{FR}$ begins at a low level, rapidly climbs to a high level then falls significantly. This behaviour is likely to trigger speculation in 


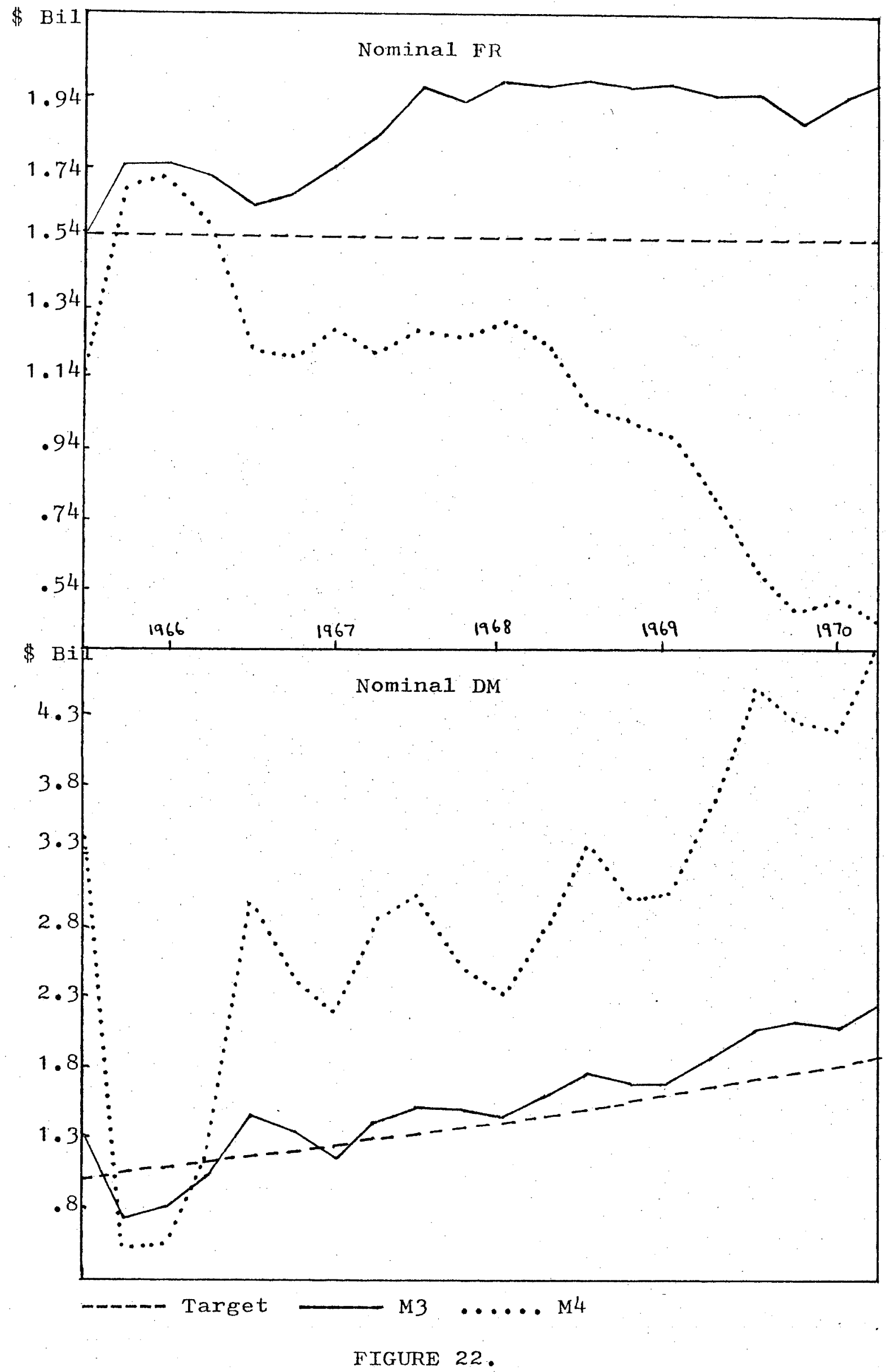


relation to exchange rate adjustments further complicating the open and monetary sectors and placing additional pressure on the monetary instrument.

The direction of $G$ away from the interest rate target has improved the income sector at the expense of the open sector. This is in contrast to experiment three where the open sector was improved at the expense of the income sector. Other experiments were performed in which the costs associated with $G$ were gradually adjusted such that the desired policy mix varied between the two extreme cases presented in M2 and M3. As fiscal policy was progressively directed away from the interest rate target (greater emphasis placed on the achievement of its own target) the optimal path for fiscal policy and real y became more stable and economically feasible. The stabilisation of the income sector had detrimental effects on the monetary and open sectors. As Y became more stable an upward pressure was placed on $\mathrm{RL}$ through an increased demand for money reflected in the nominal value of $Y$. The removal of this upward pressure entailed severe monetary policy resulting in violent. repercussions throughout the remainder of the monetary sector and the open sector. Therefore we have a trade-off between, for simplicity, internal and external balance when we wish to include $R L$ as an explicit component of the welfare function. If the rate of interest is to be treated as a target the planning authorities must decide in which sector the system and community as a whole can best tolerate instability and then apply the instruments accordingly.

\section{THE OPTIMAL ACHIEVEMENT OF THE MONETARY BASE TARGET}

The interest rate and money supply experiments indicate that while the achievement of $R L$ and $M$ targets can be consistent with a 
reasonable stabilisation of income, considerable fluctuations exist in other variables, particularly in foreign reserves. In this section, the total monetary base will be explicitly aimed for and the resulting behaviour of the system will be observed and compared with the $\mathrm{M}$ and $\mathrm{RL}$ experiments. As before, the experiments should only be viewed as exploratory polar cases as for most practical policy implementations the money base target would most likely be included in the planners' welfare function along with $\mathrm{Y}$ etc. Before proceeding directly to the experiments a number of observations on the previous performance of $M B$ should be made. Up to this stage of the study, MB has only been explicitly included in the welfare function for one experiment, IEBl. In that case the results were reasonable compared with IEB2, IEB3, IEB4 (although IEB4 gives the best result) and the RL and $M$ experiments. The respective RMSD's are given below in Table 12:

TABLE 12

$\mathrm{RMSD}_{\mathrm{MB}}$

$\begin{array}{lcc} & \text { D } & \text { S } \\ \text { IEB1 } & 169 & 184 \\ \text { IEB2 } & 516 & 743 \\ \text { IEB3 } & 376 & 775 \\ \text { IEB4 } & 99 & 124 \\ \text { M2 } & 454 & 246 \\ \text { M2 } & 130 & 168 \\ \text { M3 } & 485 & 660 \\ \text { M4 } & 1235 & 1715\end{array}$

The results show that given particular instrument weightings, the fluctuations in the optimal path of $M B$ can be quite severe. 
Surprisingly, the best overall result is obtained for IEB4 where the monetary instrument was forced to follow its desired path. The restriction of DM of course resulted in the FR target being substantially compromised. However, the combination of DM and FR produced an effectively stabilised monetary base compared with other results. The second best solution was obtained for M2 with both instruments adjusting freely. In this case, both DM and FR followed severely fluctuating time paths which largely cancelled each other out to give a stabilised total monetary base. Focussing solely on the IEB experiments one could possibly conclude that because the trade-off between FR and DM is so strong (see the structural and reduced form equations), the more vigorous monetary policy becomes the more destabilised MB becomes, even when $\mathrm{FR}$ is on target. However, the monetary target experiments show that an exceptionally vigorous monetary policy (and a destabilised external balance position) coupled with a strong fiscal policy both directed at the supply of money is also consistent with a stabilised MB (compared with other experiments). Obviously the choice of target is important but the diversity of results makes it extremely difficult, if not impossible to make any a priori assessments about the effect of stabilising MB on the remainder of the system. The results of the $M$ and $R L$ experiments were relatively predictable. In all the IEB experiments when $M$ and $R L$ were treated as intermediate variables, the achievement of $\mathrm{Y}$ and $\mathrm{FR}$ was consistent with optimal paths for $M$ and $R L$ which tended to exhibit severe movements, if not for the entire planning period at least for significant subsets. The achievement of $M$ and RL largely produced the reverse reaction, particularly in FR (depending on the particular instrument weighting used). 
for $M B$ with both instruments free to adjust. The relative weightings were as follows:

$\begin{array}{rlc}\text { MB } & \text { G } & \text { DM } \\ 2000 & 1 & 1\end{array}$

Recall that the above instrument weighting was consistent with a stabilised $Y$ when $M$ was aimed for and a destabilised $Y$ when RL was aimed for. In this case, the perfect stabilisation of $M B$ results in an effectively stabilised $Y$ as in the money supply case (see figure 23). The time path for real $Y$ is initially very close to its target, in fact for practical policy purposes it would be regarded as being perfectly stabilised up until period 12. A comparison of the RMSD's for $\mathrm{Y}$ for the money supply experiment, M2, and the first money base experiment show that a base target is inferior to an $M$ target in respect to stabilising $\mathrm{Y}$.

$$
\operatorname{RMSD}_{Y}
$$

M2 174

M45 $\quad 225$

Once again a result which would gratify the monetarists although it must be reiterated that the transmission process modelled here would perhaps differ to a monetarists conception of the world. However, the results do suggest that they could be right in a 'Keynesian' world as well. It should be stressed that this result should not be accepted as a hard and fast rule as clearly considerable additional research would need to be carried out with alternative model specifications before the general applicability of such a conclusion could be accepted. 27

The presence of a strong trade-off between $M$ and the rates of interest was. indicated by the $\mathrm{M}$ and $\mathrm{RL}$ target achievement experiments. Does the achievement of an $M B$ target result in severe movements in $M$ and 


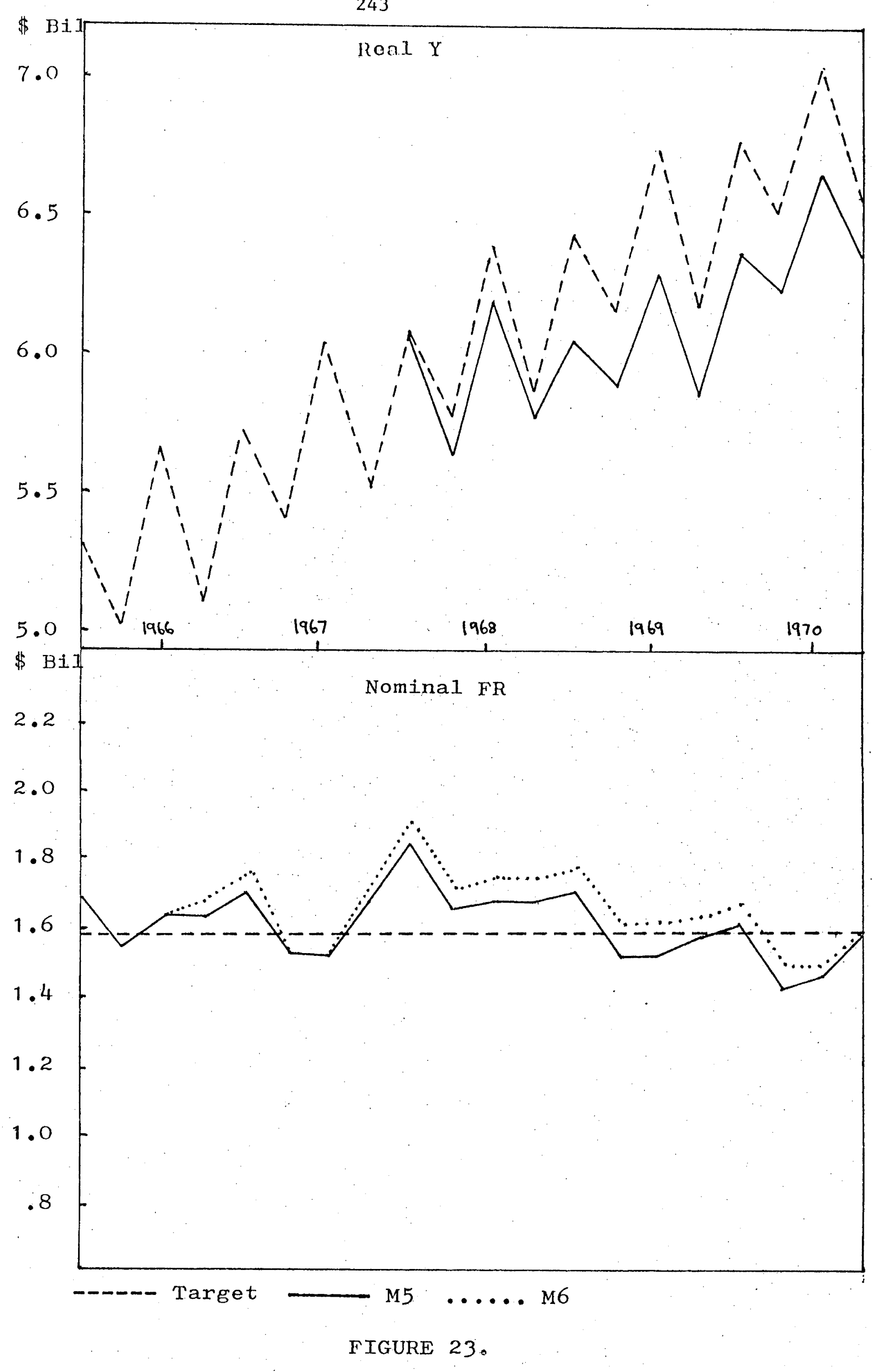


RL? Figure 24 illustrates the optimal paths for $M$ and RL. The time path for $\mathrm{RL}$ is very similar to those obtained in the IEB experiments and is less severe than the time path obtained for the money supply case when fiscal policy was substantially restricted but is clearly less acceptable than that obtained in the freely adjusting instrument, money supply case. The problem of financial and asset market stability once again becomes important and if it was deemed to be desirable to eliminate excessive swings in the rate of interest then $\mathrm{RL}$ would need to be explicitly included in the cost function but not as the sole component as we have seen that the exact achievement of an interest rate target can be destabilising. The optimal money supply path is effectively stabilised for the first half of the planning period but begins to contract in period 12. This contraction is not brought about by the money base, obviously, but results from a downward movement in the short rate of interest. The result illustrates a dilemma of monetary policy in that even if the authorities are able to successfully stabilise the total monetary base, a smooth monetary expansion will not necessarily result due to the profit maximization response of the trading banks to the rate of interest. ${ }^{28}$ The presence of a strong trade-off between $M B$ and RL will naturally result in one variable being sacrificed unless both are explicitly aimed for and even then they can only both be achieved in a strongly-Tinbergen world. To enable both targets of $\mathrm{MB}$ and $\mathrm{RL}$ to be achieved in a fixed target framework we require that the rank of the matrix of instrument multipliers be equal to two. The appropriate matrix is given by

$$
\left[\begin{array}{cc}
.0512 & .7453 \\
.0007 & -.0003
\end{array}\right]
$$




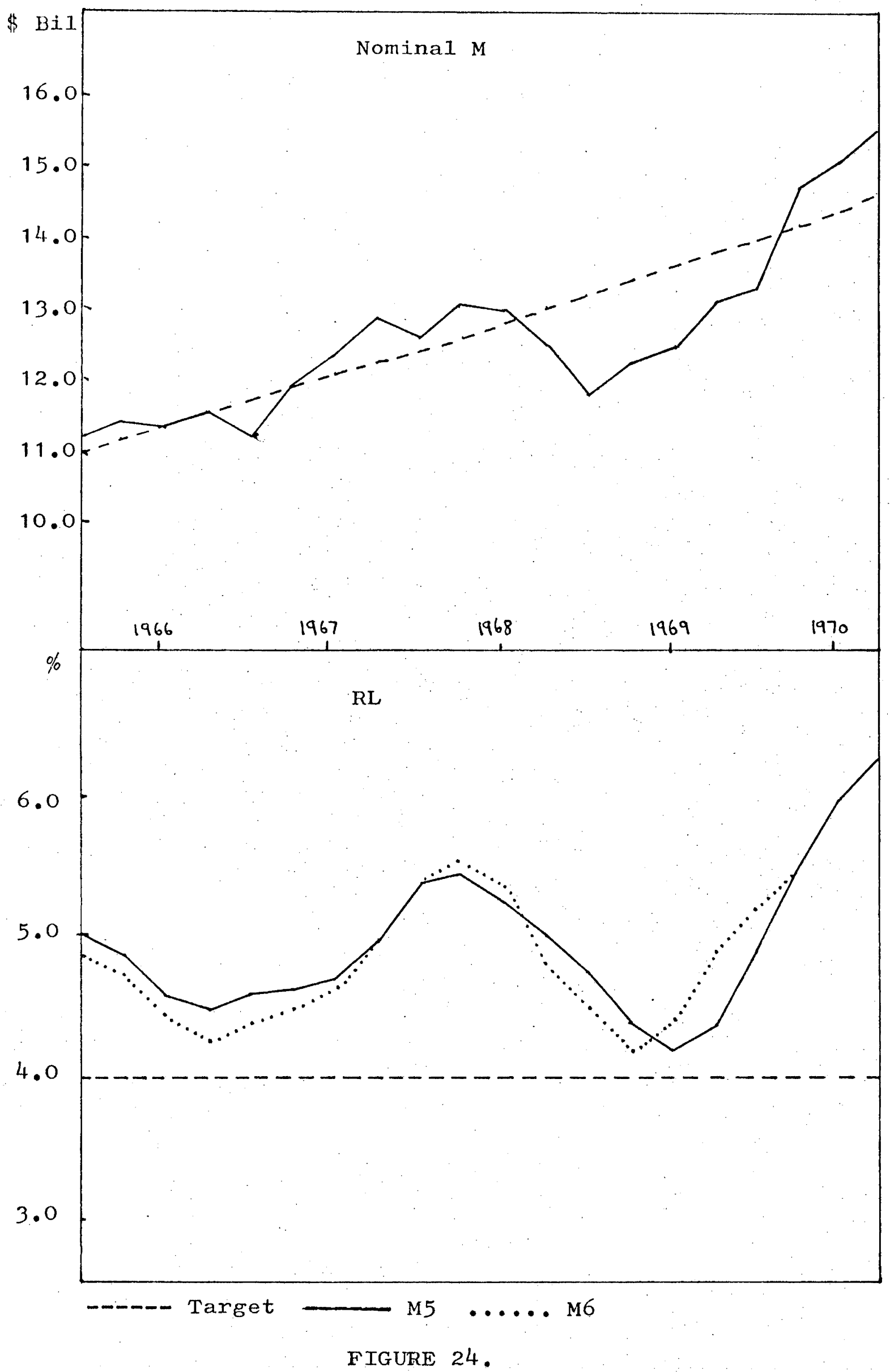


which has rank equal to two. Solving for both targets in the fixed target framework yields results which are totally unacceptable, for example, the RMSD for $\mathrm{Y}$ is 1076 which is completely destabilising compared with the RMSD results listed earlier. In addition, the required values of the controls are not feasible with government spending taking on negative values in some quarters. The results suggest that the monetary authorities can do little to overcome the dilemma of a smooth base time path and a fluctuating interest rate time path. 29 The more closely the two variables are controlled, the more destabilised becomes the rest of the system. A smooth money supply expansion can only be obtained by allowing both. $M B$ and the rate of interest to freely adjust in a compensatory manner.

The other endogenous variable of importance is FR and the optimal path for this variable is certainly worse than all the IEB experiments as could be expected (remember that a different scale on the vertical axis was used for the IEB experiments) with large fluctuations which would most likely produce speculative capital flows. The RMSD for FR is 95 while the RMSD for the 'best' FR from the money supply and rate of interest experiments was 93 - a negligible difference. We have then a situation in which the achievement of a money base target is consistent with a stable income sector and a fluctuating external balance sector which is certainly not totally destabilised and no worse than the best result obtained from the achievement of other monetary targets. Is such a target configuration feasible in terms of the policy required to achieve it? The answer is yes, and indeed, the optimal policy $\mathrm{mix}$ required to achieve the target exhibits some particularly interesting characteristics. Firstly, fiscal policy has been entirely neutral in that for the entire planning period it was almost exactly on target even 
though it was left free to adjust. The reason for this can be found in the reduced form where it can be seen that the impact of $G$ on $M B$ is equal to 0.0512 while the impact of DM is equal to .7453 and as such monetary policy completely swamps fiscal policy. In addition, to ensure policy uniqueness, G and DM were assigned positive weights (although extremely small) and as the impact of $G$ is so insignificant, for $G$ to be active would have required an exceptionally severe policy (and probably not feasible) over the entire planning period thus creating high welfare costs even with a low weight. The net result has been a passive use of fiscal policy. Under these circumstances it would not be unreasonable to conclude that in the absence of any assistance from fiscal policy, monetary policy would be used in a very severe fashion as it was under similar conditions in previous experiments. Figure 25 indicates that this is not the case and indeed the optimal monetary policy required for this experiment is the least severe of all the monetary experiments. To enable a comparison with the IEB experiments, the optimal DM has also been graphed with the same scale on the vertical axis as in the IEB experiments. A comparison of this graph and the first four IEB optimal monetary policies reveals that the time path for DM for M5 is less severe than the policies to achieve external balance. It should be clear that the next experiment with G fixed is largely redundant as it could reasonably be expected that the results will be very similar to $M$ due to the passive nature of fiscal policy in that experiment. The cost function weighting for the final experiment was as follows:

$\begin{array}{ccc}\text { MB } & \text { G } & \text { DM } \\ 2000 & 1000 & 1\end{array}$

and indeed the results are almost identical to the previous experiment as is shown by the following RMSD's. (deterministic). 


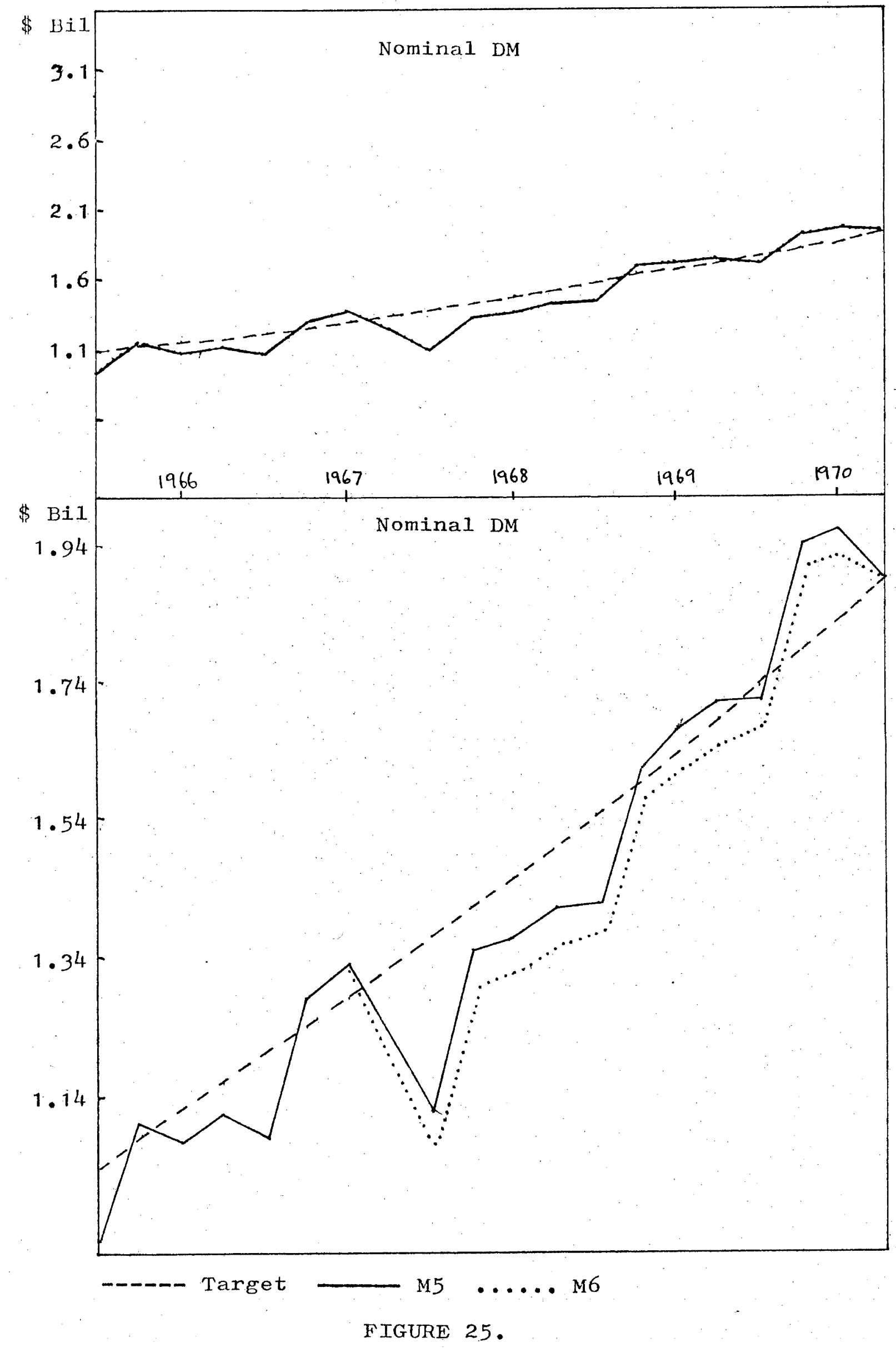


TABLE 13

\begin{tabular}{lcc}
\multicolumn{2}{c}{} & \multicolumn{2}{c}{ RMSD Comparisons - MB Target } \\
& RMSD $_{\text {M5 }}$ & RMSD $_{\text {M6 }}$ \\
Y & 381 & 353 \\
FR & 95 & 110 \\
C & 370 & 365 \\
M & 587 & 600 \\
RL & 1.1 & 1.1 \\
CF & 101 & 102 \\
G & 25 & 0.0 \\
DM & 95 & 115
\end{tabular}

The important result to come out of the money base experiments is that MB can be forced to follow a steady growth path which is consistent with a stable income sector and an external balance sector which is not as acceptable as the optimal FR's obtained in the IEB experiments but is not totally destabilised and is as good a result as the 'best' $F$ R obtained in earlier monetary experiments. Furthermore, to achieve the base target the optimal monetary policy required to hit a money supply or interest rate target and as such the excessive shifts in domestically created liquidity have been eliminated implying greater stability in the composition of the private sectors' portfolios. One of the reservations associated with earlier experiments was that the optimal monetary policies were of dubious feasibility due to the implication that the private sector would need to shift rapidly between extreme portfolio compositions, that is, from virtually no assets to saturation levels and vice versa. This dilemma has been eliminated. The absence of any significant fiscal policy effect is also important as it allows the monetary authorities to independently aim for an MB target regardless of what the fiscal 
authorities are doing in the income sector. Hence, the phasing and complementarity of monetary and fiscal policy becomes less important in this case. This is certainly the reverse of the money supply and rate of interest examples. The insignificant feedback of the income sector to the monetary base resembles the PR framework mentioned earlier and it is clear that only in the case of a dominant monetary policy and passive fiscal policy is it reasonable to treat the income sector as exogenous in relation to the monetary sector. The analysis carried out above also illustrates that it is only feasible to treat income as exogenous in relation to a subset of the monetary sector as it is clear that the influence of $\mathrm{Y}$ and $\mathrm{G}$ can vary between individual variables within the monetary sector itself.

\section{INSTRUMENT INSTABILITY}

The monetary experiments are very amenable to an analysis of instrument instability within the framework of Chapter 4 (instability with one target) as in each case only one target is explicitly included in the cost function. Of particular interest is the result that in only one case are predictions summarised in Table 5 of Chapter 4 realised in the six single target experiments discussed in detail. That experiment is the achievement of the money supply target with $G$ fixed and DM free. A surprising result is that the significant downward explosive nature of the monetary instruments associated with the $\mathrm{MB}$ target and detected in the instrument instability experiments has been eliminated. Instead, the optimal monetary policy is the most stable of all the policies required for experiments $\mathrm{Ml}$ to $\mathrm{M6}$. The switch from a severely unstable to a stable behaviour once again illustrates the problems facing policy planners. The underlying structure suggests that the monetary instrument is 
inherently unstable when assigned exclusively to a monetary base target but the inclusion of uncontrollable exogenous influences and the specification of a numerically increasing target path has resulted in the instrument appearing to be stable. The implications of this are of considerable importance as policy planners may well be inclined to weight the potentially unstable instrument to avoid any explosive behaviour. This action would be unnecessarily restrictive and would prevent the achievement of the desired target when the target could be exactly achieved with non-structural influences forcing the instrument of concern to act as if it were stable. ${ }^{30}$ The behaviour of DM in relation to the rate of interest is also important and lends support to the conclusions obtained in the preceding chapter. The analysis of Chapter 4 produced the result that DM is structurally stable with $G$ fixed in relation to $R L$, allowing for a four period adjustment at the beginning of the planning period to compensate for the influence of the initial conditions. The results obtained in this chapter indicate that DM is used in a very severe manner in relation to $\mathrm{RL}$ and gives the appearance of instability and could lead to the weighting of DM for an incorrect motive. The stability results briefly discussed in this and the previous chapter illustrate the importance of disentangling pure structural instability from induced instability or stability. A failure to do so could lead to completely inappropriate responses by the planning authorities.

\section{STOCHASTIC EXTENSIONS}

As with the internal and external balance experiments, the monetary experiments were carried out in a stochastic framework in addition to the deterministic framework already discussed in some detail. The results of the stochastic monetary experiments deserve some attention 
as the structure of the model could have an important influence on the stochastic responses of the system. The structure of the model excludes lagged effects of the supply of money and the monetary base. Thus, the optimal controls will not adjust to past shocks in those variables but to past shocks in other possibly largely unrelated variables. This is in contrast to the situation in the IEB experiments where both $Y_{t-1}$ and $\mathrm{FR}_{t-1}$ figured predominantly in the feedback matrices and $G$ and $D M$ were able to adjust to past shocks in the target variables. Tables 14 to 16 contain the feedback matrices for selected periods for experiments M2, M4, and M5. Note that for M2 (money supply target with both instruments free) the influence of past state variables on the controls is spread fairly evenly indicating that shocks from all sectors of the model could have a reasonably significant effect on the stochastic adjustment of G and DM. As in Chapter 5 the magnitude of variables needs to be considered when feedback influences are being discussed. Of particular interest is the influence of $\mathrm{RS}_{t-1}\left(\mathrm{RS}_{t-4}\right)$ and $\mathrm{RS}_{t-1}$ on both controls which is probably the most significant of all. The close relationship between $M$ and the rates of interest which was apparent in the earlier discussion of the monetary experiments means that any movement in the short rate will have a definite distinct influence on $M$ of a substantial degree which leads quite naturally to adjusting the instruments in response to past movements in RS. $\mathrm{FR}_{t-1}$ also plays an important role in the formulation of numerical values of the controls which once again is a reasonable result due to the influence of $\mathrm{FR}$ on the total monetary base. While the feedback effects are apparently spread fairly evenly over the whole range of lagged state variables, the behaviour of particular coefficients over the planning period is of considerable interest. Firstly, while the lagged effect of income consistently has a positive effect on $G$, lagged 


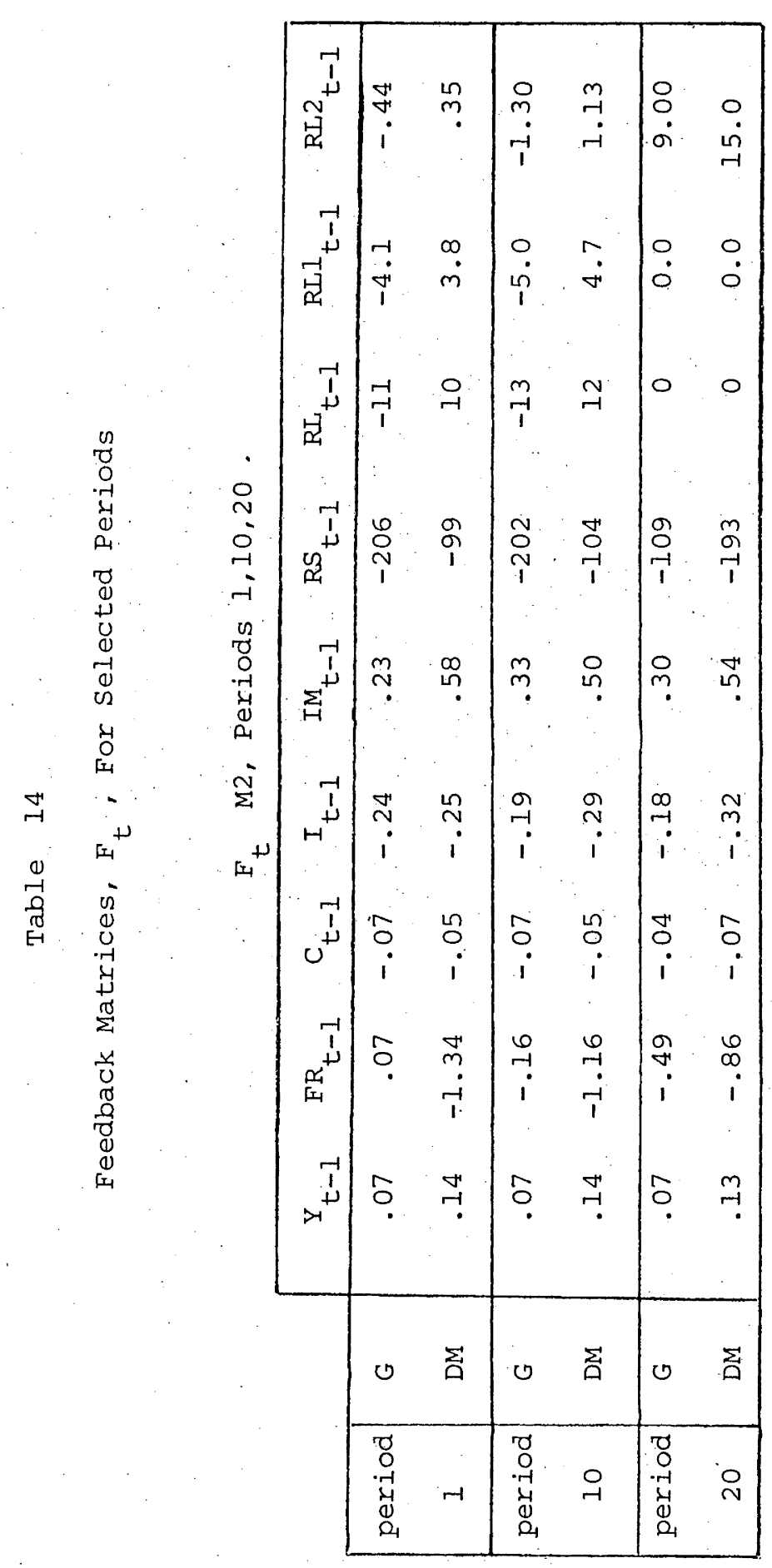

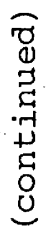




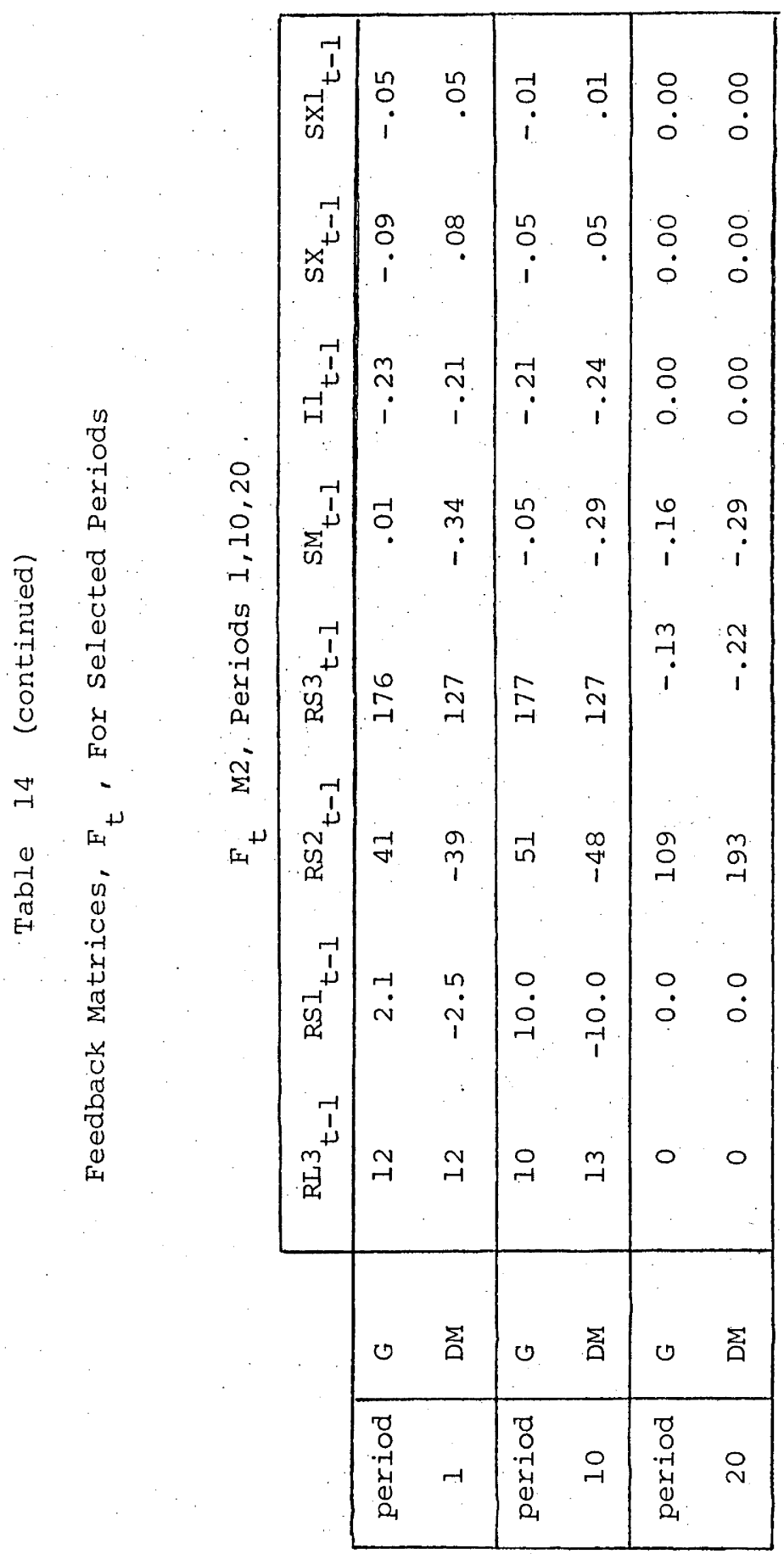


$C$ and I have negative effects on G. The effect of this is as follows. An increase/decrease in $\mathrm{Y}_{t-1}$ will generate an upward/downward movement in $G$ while the reverse will occur in $C$ and $I$ and as $C$ and $I$ are components of $\mathrm{Y}$, the income effect on $G_{t}$ could be negated depending on the relative magnitudes of variables at time $t-1$. Thus, while income variables appear in the feedback matrices, the response of the controls will be more dependent upon the monetary and open sectors if the income effects are in fact negated. (The income sector will of course influence the behaviour of the omnetary variables through the system structure.) Secondly, the coefficient of $\mathrm{FR}_{\mathrm{t}-1}$ behaves in a strange manner in relation to $G_{t}$. Initially, $F R_{t-1}$ has a positive impact on $G_{t}$. Thus, if $\mathrm{FR}_{t-1}$ has fallen, presumably reducing $M B$ and $M$, then $G$ is increased the next period which will force $\mathrm{Y}$ up and hence $M$ through the demand for money and interest rate mechanism. However, as can be seen from Table 14, the effect of $F R_{t-1}$ is reversed in later periods with a decrease in $F R_{t-1}$ leading to a decrease in $\mathrm{G}$ and ceteris paribus a decrease in $\mathrm{Y}$ and $\mathrm{M}$. Switching effects are largely the result of the target specifications and the scaling of weights to eliminate cost function bias. ${ }^{31}$ such behaviour of the feedback coefficients causes no problems in a world of certainty but could be important when stochastic shocks are introduced or accurate forecasts of the exogenous variables have not been made with the net result that the targets may no longer be feasible (especially where identities are concerned) and the correct current and immediate past state vector cannot be accurately predicted. Under such conditions a modification of targets may be required or a change in the sign of the previously computed feedback coefficients may direct the system away from the desired position. The change in magnitude and sign of the feedback coefficients once again illustrates the difficulty in trying to 
empirically estimate short-run historical feedback matrices. The convergence of $\mathrm{F}_{t}$ to a steady state solution would provide a more amenable framework for such an analysis but the use of multiple cost matrices precludes this. In any case, historical policy has tended to be constructed on a short run basis and a steady state approach to its analysis may be inappropriate. It would appear to be advantageous to have lagged values of each endogenous variable explicitly included in the structure of the model to ensure that the instruments always respond to past movements in the target of concern (of course the degree of response will largely depend on the importance of the lagged variable in the system). The feedback matrices presented in Table 15 illustrate the importance of the presence of lagged values of the variable being controlled. It can be seen that the lagged value of the long rate completely dominates the formulation of the optimal monetary policy although $\mathrm{FR}_{\mathrm{t}-1}$ is of some importance also. With the introduction of additive disturbances, it would appear to be certain that the monetary instrument would adjust in a completely complementary manner to past shocks in $\mathrm{RL}$ with the result that the long rate would track very close to its target. The response of $G_{t}$, in contrast, is spread more evenly over the entire range of lagged state variables. Also note that the switching effect is not present in this case. Finally, Table 16 contains the feedback matrices for the case where $M B$ is the target, and as $\mathrm{MB}_{t-1}$ does not appear in the model, the controls will only respond to structural disturbances in other, possibly largely unrelated, variables. As with the case of a money supply target, the feedback effects are distributed more evenly than in the rate of interest case and once again the switching of feedback effects occurs although, not in such a serious way compared with the coefficients in Table 14 . 


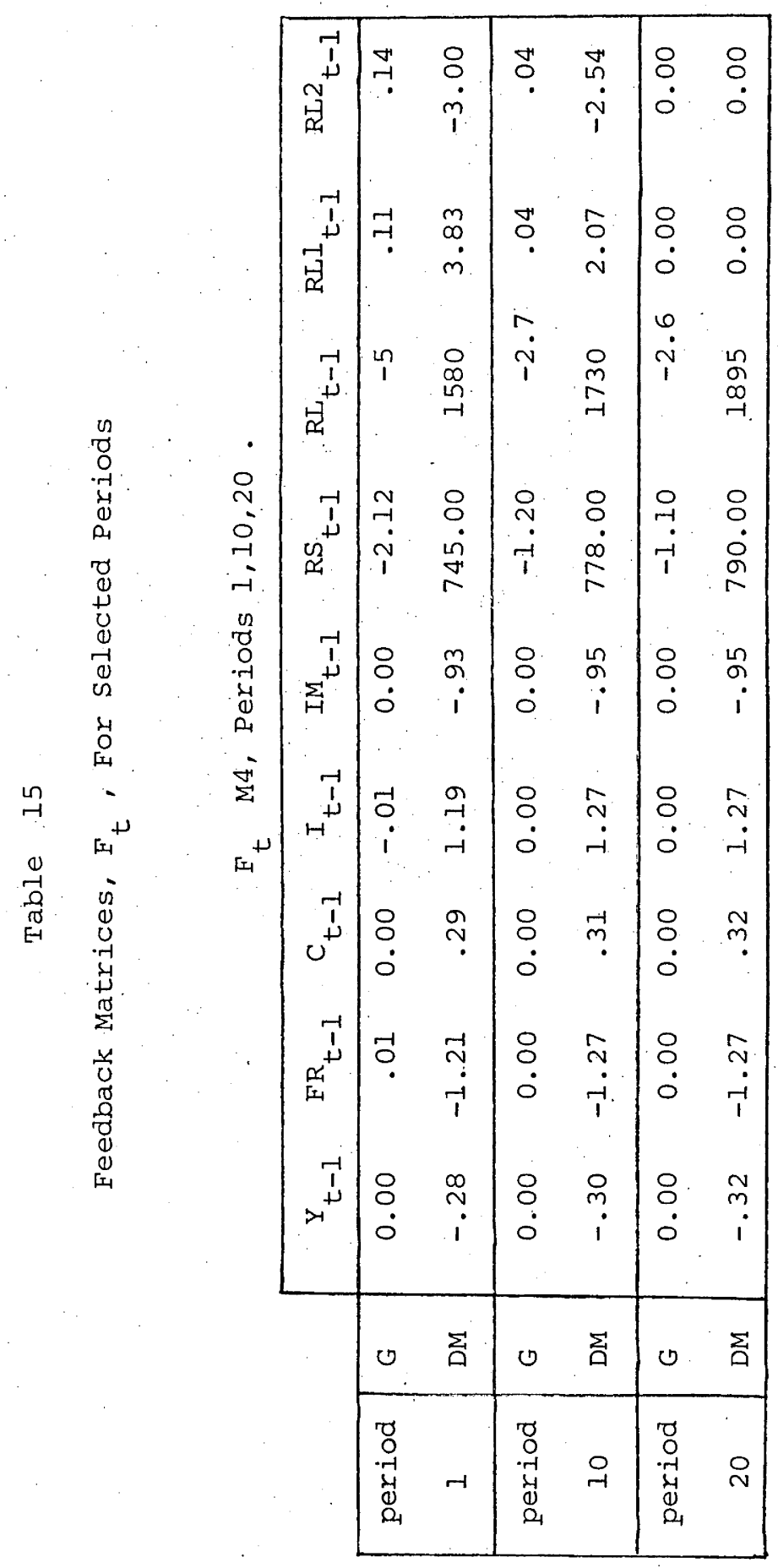

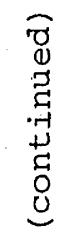




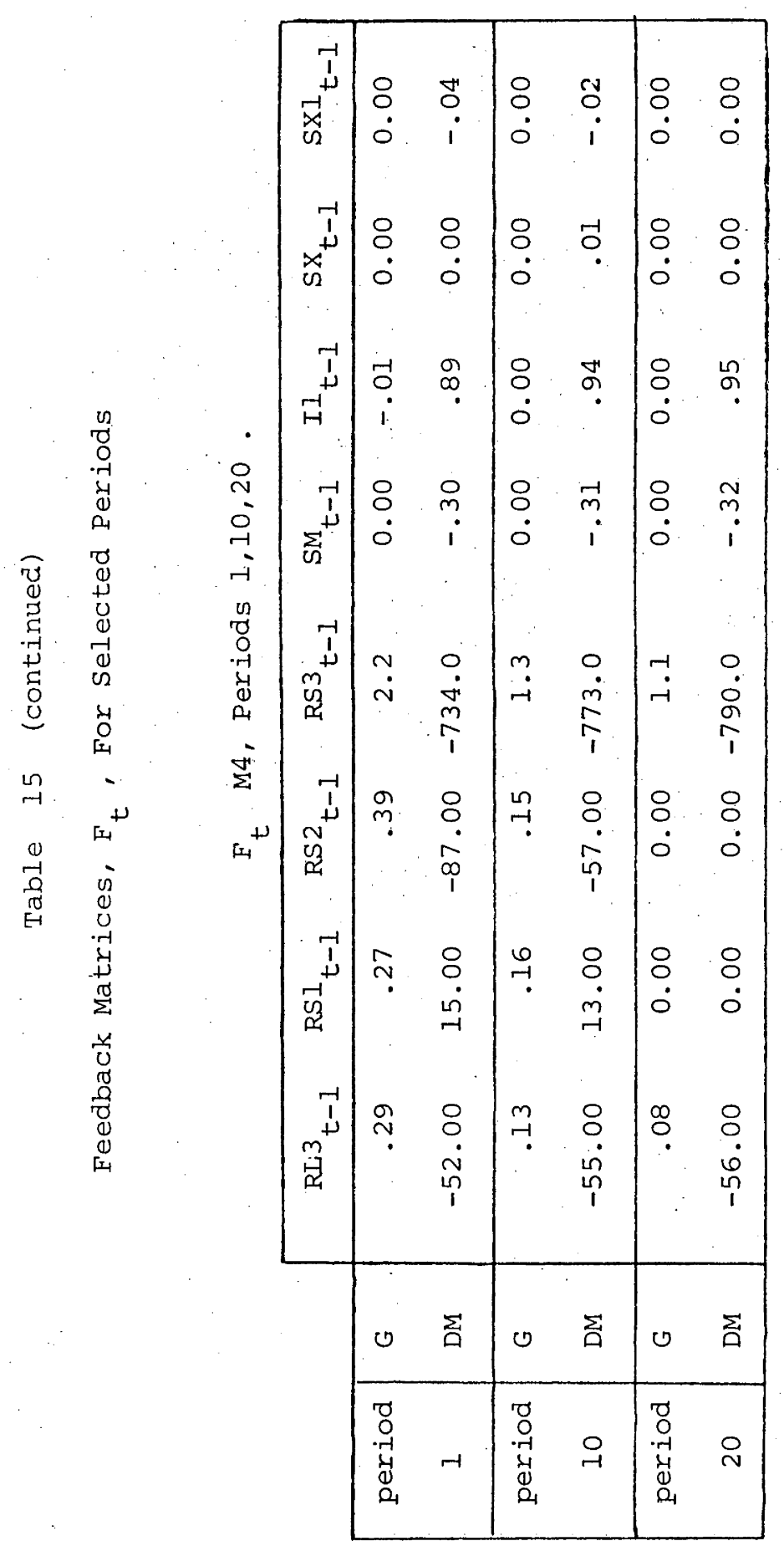




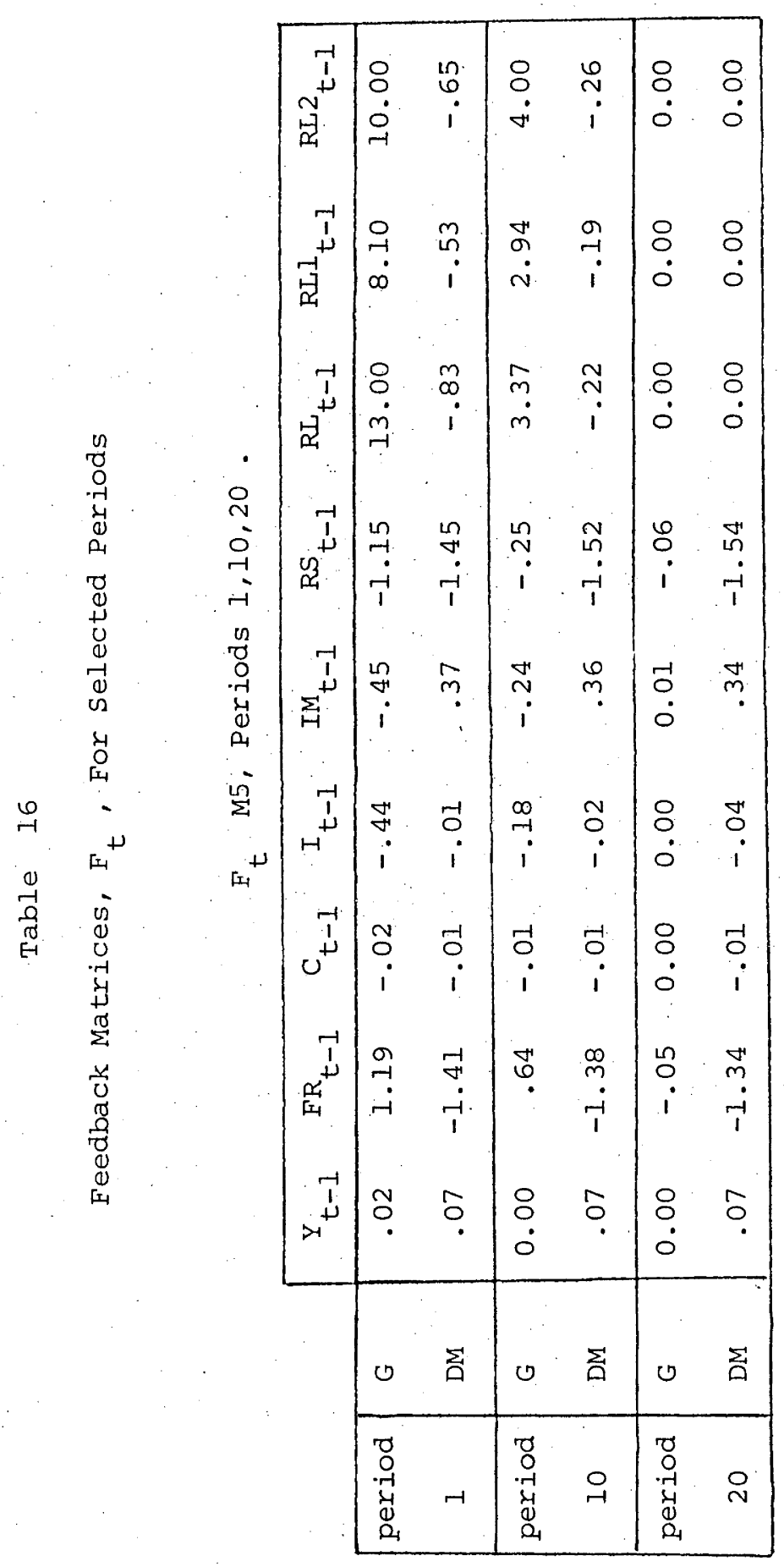

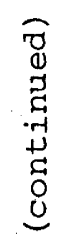




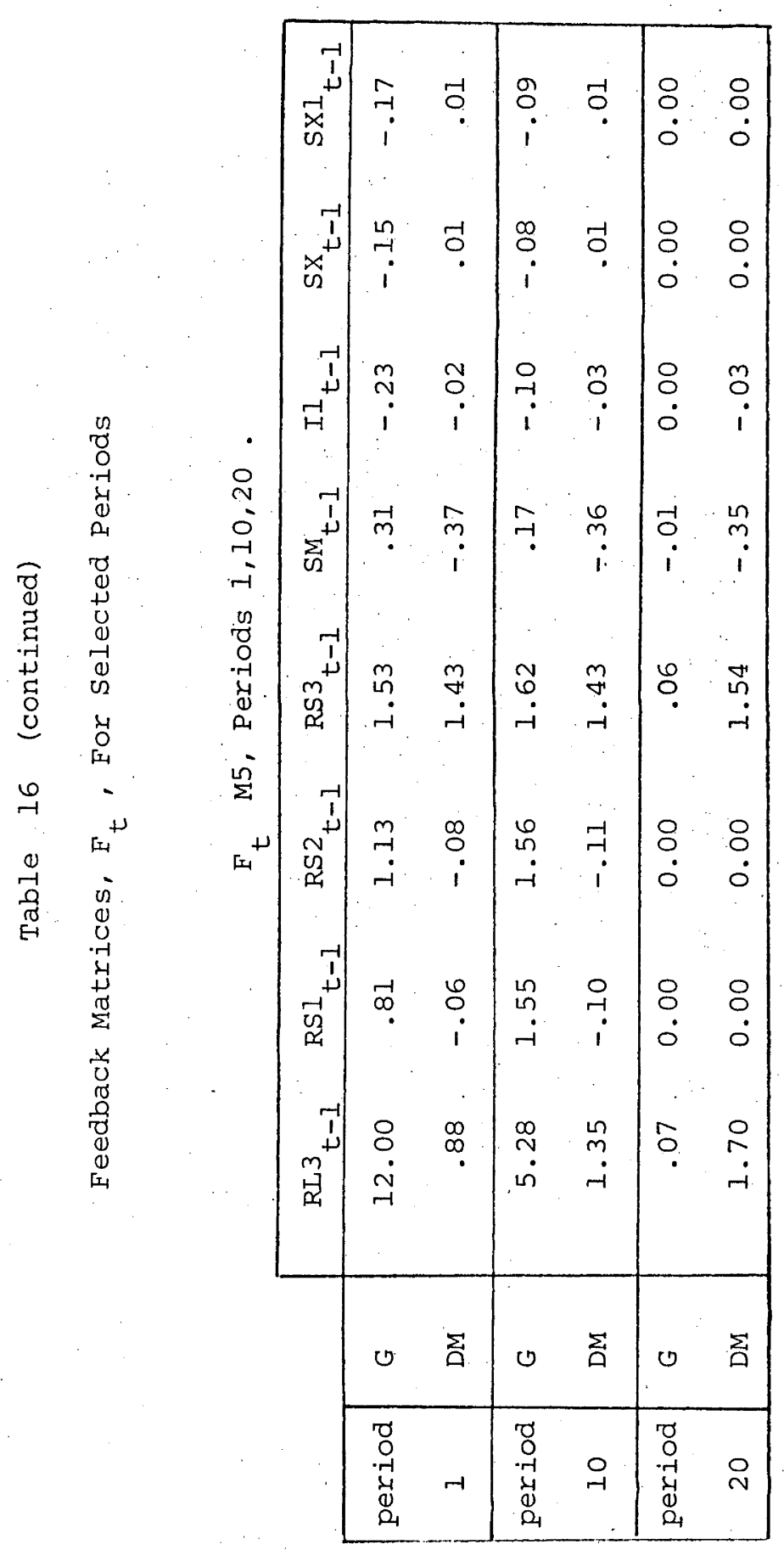


Keeping in mind the properties of the relevant feedback matrices, we now turn to a stochastic analysis of the monetary experiments. For illustrative purposes only, the same set of single shocks, as opposed to monte carlo repetitions, as those employed in the IEB experiments will be used. The results are graphed in Figures 26 and 27 . The results confirm the analysis of Chapter 5 in that the optimal control laws are selfadjusting to stochastic disturbances in a manner which is complementary to the achievement of the relevant targets. In particular, the absence of lagged values of the target has not hindered the achievement of optimal paths for $M$ and $M B$ which fluctuate about their respective targets. We know from previous experiments that FR in particular is prone to react adversely to additive disturbances but this has not resulted in severely destabilised time paths for $M$ and $M B$ even given the switching of feedback effects. However, the stochastic time path for $M$ does exhibit some large percentage deviations from its target whereas the time path for RL tracks much closer to its target. This is best illustrated by comparing the welfare loss for both variables. The welfare loss for $M$ in $M 2$ is 10.73 while the welfare loss for RI in M4 is 7.94. (Note that the use of time varying cost matrices and exact scaling of weights to a common bench-mark makes a comparison of welfare feasible even between variables of such divergent magnitude.) The results illustrate the extremely robust nature of the linear/quadratic technique in that the presence of additive disturbances need not be destabilising even when the controls are adjusted to shocks in variables which have little influence on the target of concern 32 This adaptability in adjustment is a feature which is absent from the dynamic fixed target approach to stabilisation.

An analysis of the behaviour of the remainder of the system under uncertainty is also important. In particular, it is desirable to 


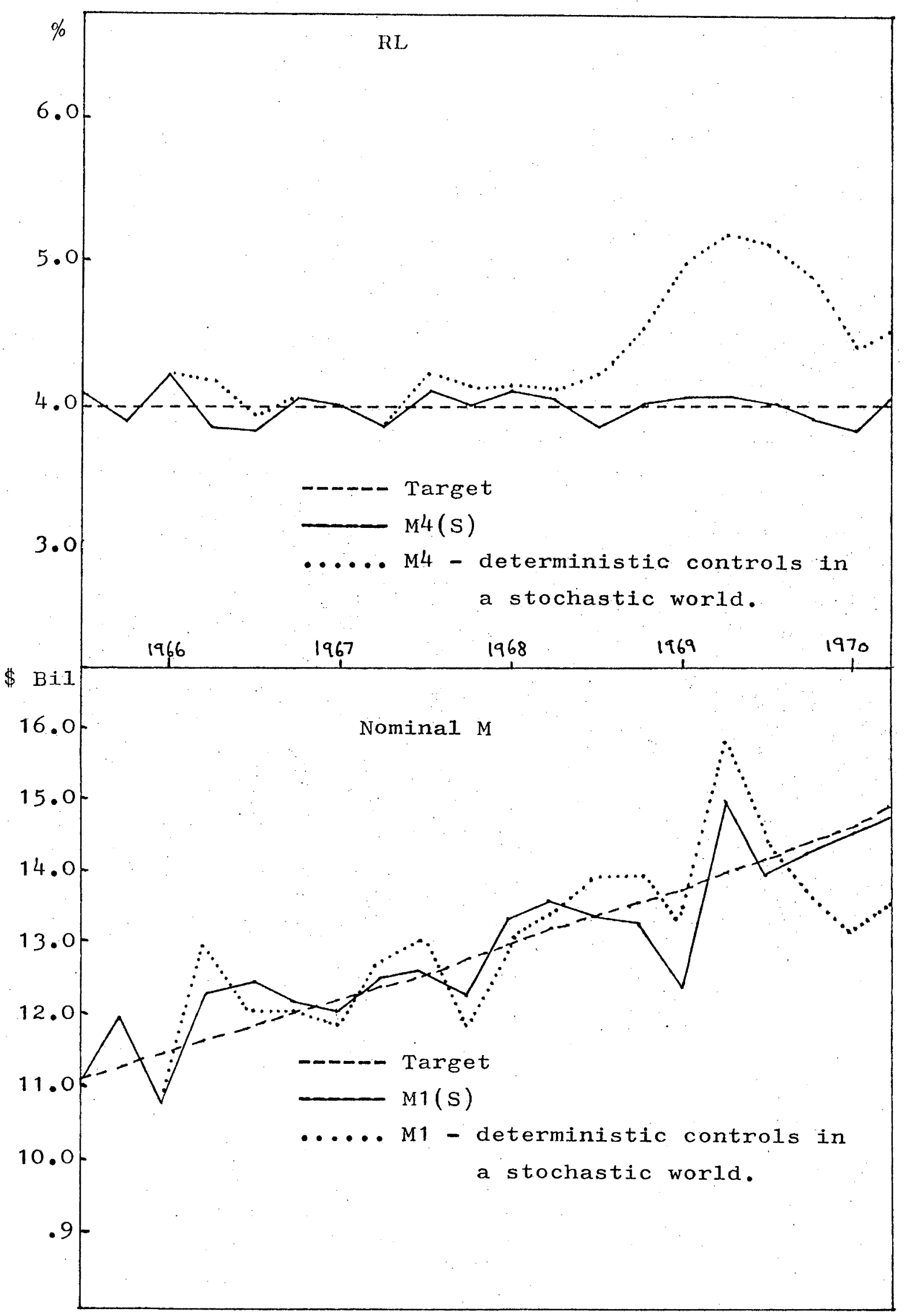

FIGURE 26. 


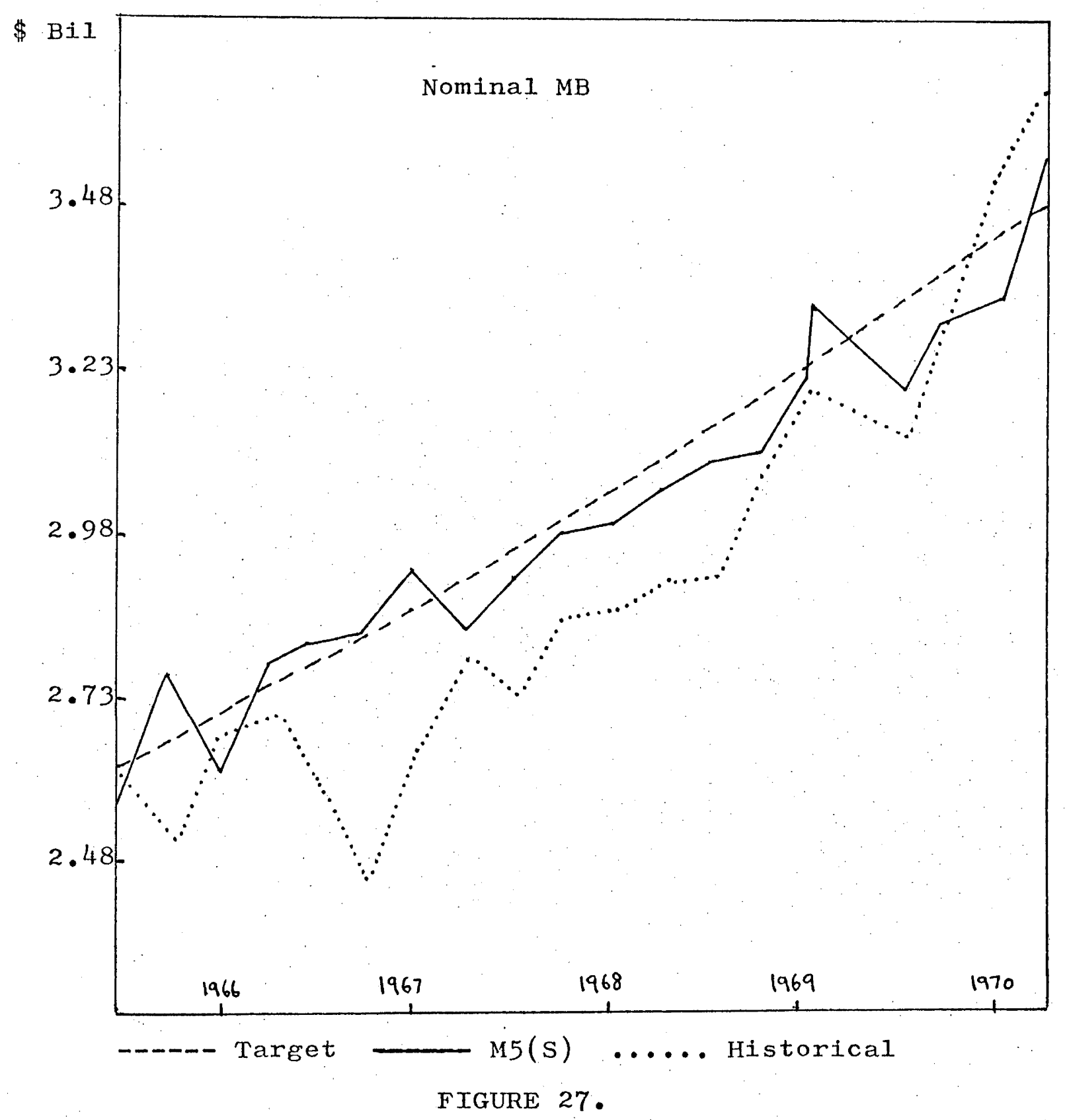

Note: The time path for MB with deterministic controls in a stochastic world is almost identical to the purely stochastic case where the instruments are allowed to adjust. 
ascertain if the presence of uncertainty results in a reversal in the performance of the state variables not incorporated in the cost function. of course, it could be argued that any change in the bahaviour of the non-weighted variables is of no concern - if it was then those variables would be explicitly included in the cost function. However, it should be remembered that the experiments carried out in this chapter are meant to be polar cases designed to illustrate what would happen to the remainder of the system if indeed monetary targets were aimed for. That is, is the achievement of a monetary target feasible in terms of its effect on the rest of the system? In this context a comparison is valid. A comparative summary of the deterministic and stochastic results is given in Table 17 .

The results broadly indicate that the basic trade-offs have been retained within the stochastic framework and although the monetary targets have been able to adequately adjust for past shocks, the remainder of the variables have in general performed in an unsatisfactory way in comparison with the deterministic results. Two notable exceptions to this are $M B$ in $M I$ and $Y$ in $M 3$. The optimal stochastic path for $M B$ actually is a substantial improvement over the corresponding deterministic path. Similarly, there is a slight improvement in the performance of $\mathrm{Y}$ in $\mathrm{M} 3$ when uncertainty is introduced. The improvement in $\mathrm{Y}$ does not represent a major shift in system performance or change in relative performance of the system between experiments as the resulting time path is still far more severe than time paths for other experiments and of a nature which would not be regarded as feasible. When the improvements in $\mathrm{Y}$ and $\mathrm{MB}$ are viewed in the context of the overall results it is clear that no adjustment in the choice of monetary target on the grounds of significant system responses would be made. That is, a money supply target would still be preferable to a rate of interest target in 


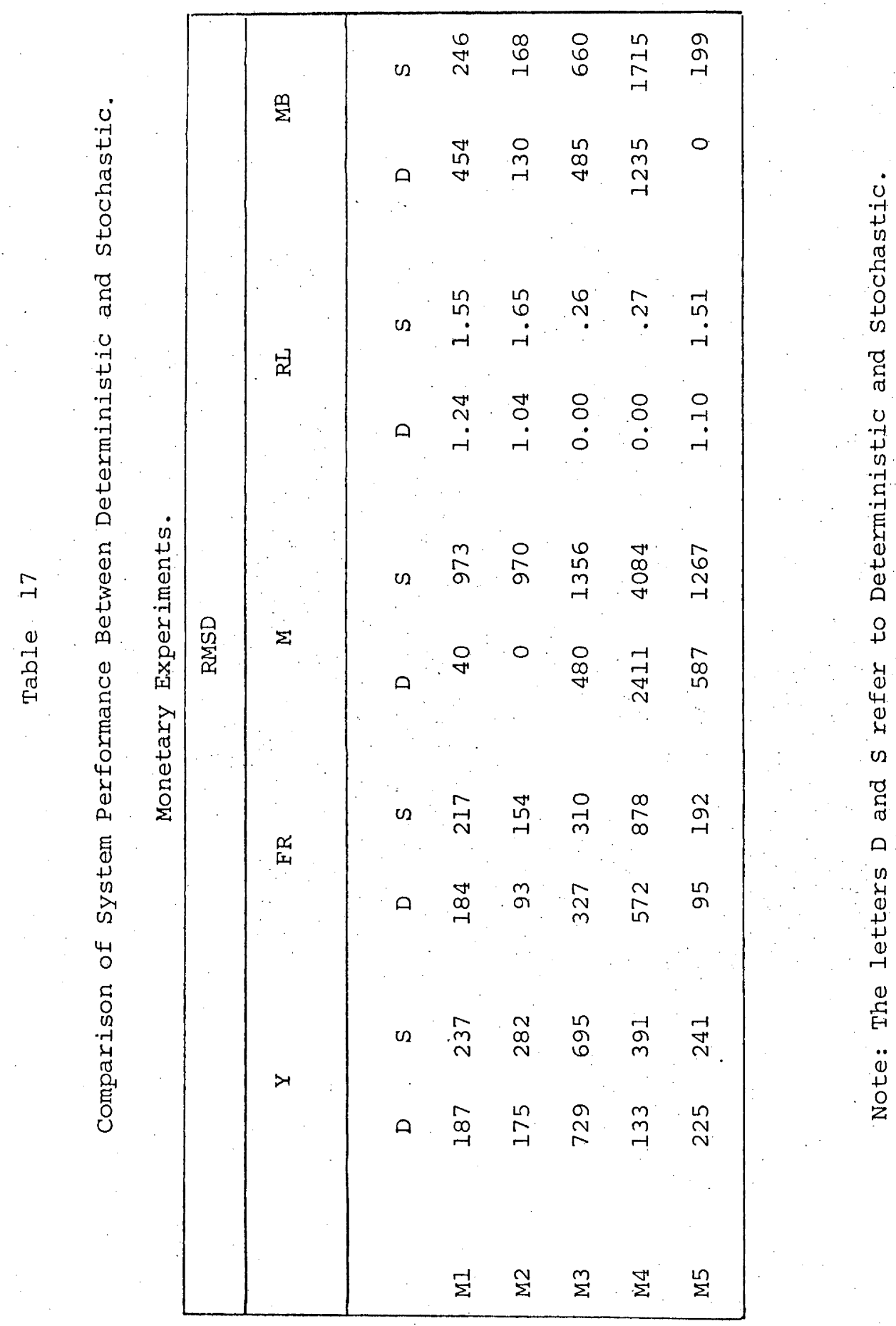


relation to the variability in $\mathrm{Y}$ and $\mathrm{FR}$ which occurs when uncertainty is introduced. Further research needs to be conducted using parameter uncertainty to ascertain whether or not uncertainty can induce a swi.tch in the choice of monetary target to be aimed for. At the theoretical level, Turnovsky (1975) has shown how the introduction of parameter uncertainty can result in a switch in the choice of monetary instrument and while it must be conceded that the empirical work carried out here falls outside of the instmument problem it should be remembered that some similarities do exist (indeed Turnovsky recognises that the instruments employed in his study are really proximate targets) between the instrument problem and the choice of proximate target. The theoretical work then, provides some foundation for more extensive empirical research on this topic.

The analysis of the preceding chapter showed that under certain conditions of uncertainty, a significant switch in the emphasis of instruments could occur from a certainty situation. The results in Chapter 5 indicated a significant switch in both instruments, except when either instrument was explicitly prevented from deviating from its target path. The stochastic monetary results, while generally supporting that contention, do not reveal such a significant effect particularly in the use of the fiscal instrument. The result of most importance is the relaxation in the use of monetary policy in $\mathrm{Ml}$ where the variation in DM about its desired path fell by 50 per cent from the deterministic results. This represents a clear switch in the use of monetary policy but it is not compensated for by a corresponding increase in the use of fiscal policy. It can be concluded from this that uncertainty has been complementary in relation to the use of monetary policy, that is, the money supply can be stabilised very closely to its target (see Figure 26) without resorting 
to the severe and possibly unobtainable monetary policy required in the deterministic case which leads to the surprising result that the required policy becomes more feasible under uncertainty. This certainly is not a general proposition but the results indicate the possibility and is a result in the same vein as that obtained by Macrae (1972) who demonstrated that the use of learning techniques in the formulation of control policies may lead to a loss of stabilisation compared with a situation of no learning. One would generally assume that certainty and learning would be preferable to uncertainty and no learning. The development of formal optimisation procedures for selecting policies has begun to reveal the fact that policies or situations which appear to be counter-intuitive are in fact 'best'. A further example of this type of result can be found in Turnovsky (1975) where it is demonstrated that a pro-cyclical monetary policy is optimal - once again a result which appears to be counter-intuitive on the surface and would most likely not be made by a policy planner deciding on policy without any formal optimisation or even non-optimisation control technique.

The stochastic responses of the instruments in the remaining experiments are predictable. In each case the introduction of uncertainty leads to a more vigorous use of policy with the greatest variation occuring in the monetary instrument. As with the IEB experiments, situations occur in which the direction of policy is almost reversed from the deterministic case particularly in M2 and M4. This would suggest that the implementation of controls computed under conditions of certainty would be disastrous if implemented in an uncertain situation. To test this assertion, the six monetary experiments were repeated with the modification that the deterministic controls were used in a stochastic simulation. The results are graphed in Figure 26. Note that the time 
paths for MB have not been presented. This is because the stochastic results and the deterministic controls in a stochastic world framework results are almost identical. Once again a surprising result in view of the fact that a significant switch in the optimal mix of policy occurred from the deterministic to the stochastic case. One could conclude that particular shocks to the system are largely complementary or minimised by the system structure in relation to the $M B$ target, thus enabling the effective stabilisation of the system with deterministic controls. Further experiments were conducted with different sets of randomly generated additive errors with the results largely duplicating the illustrative example presented. In any case, the example is sufficient to provide a counter example to any generalised statements along the lines of "the formulation and execution of policy under supposed conditions of certainty will be disastrous or destabilising in an uncertain world". The money supply results are also of a similar nature although not to the same degree as the $M B$ results. Figure 26 reveals that for most periods (with the exception of the final three) the money supply tracks very closely to the purely stochastic optimal path. This implies that the restriction placed on fiscal policy has a nil effect on the performance of the system when deterministic controls are implemented in an uncertain world. In contrast to $M$ and $M B$, the sub-optimal path of RL with deterministic controls in a stochastic world, diverges significantly from its purely stochastic counterpart and the desired target. The time path is initially very close to the RL target but begins to systematically diverge about half way through the planning period. Once again, the difference between purely stochastic results are negligible. As a result $\mathrm{M} 3(\mathrm{~S})$ has not been graphed. The behaviour of RL can be traced back in part to the original structure of the 
system where the lagged effect of RL plays a significant role in the long rate equation. Thus, the high level of dependence of $\mathrm{RL}_{t}$ on $\mathrm{RL}_{t-1}$ can result in shocks in $\mathrm{RL}_{t-1}$ moving $\mathrm{RL}_{t}$ off target and even in the absence of any future shocks, it could be expected that $\mathrm{RL}_{t}$ will continue to systematically diverge from its target. The presence of additional shocks, depending on the size and direction, could be expected to keep $\mathrm{RL}_{t}$ away from its target in the absence of any compensatory movement in the instruments. The results of the purely stochastic experiments show quite clearly that if the instruments are free to adjust to past shocks then the presence of uncertainty has an almost negligible effect on the behaviour of $\mathrm{RL}_{t}$. The excellent compensatory stochastic instrument adjustment is a direct result of the dominance of $\mathrm{RL}_{t-1}$ in the appropriate feedback matrices (see Table 15). A direct comparison can be made between the $\mathrm{RL}$ results and the results obtained for FR under the same conditions in the IEB experiments. It was found that FR behaved in a similar manner to $\mathrm{RL}$ (stochastic with instrument adjustment) due to the presence of $\mathrm{FR}_{t-1}$ in the identity for $\mathrm{FR}_{t}$. The results would seem to suggest that the implementation of controls derived from conditions of certainty in an uncertain environment could be destabilising if the target(s) of concern is/are a function of the lagged target, with the qualification that the lagged dependent variable has a coefficient greater than say .5 . The generality of such a proposition cannot be established from one applied model but the results seem to be important enough for more research to be carried out as it is clear that if control techniques, and in particular the linear/quadratic technique, are to be used as an aid for policy specification by governments who will not have complete knowledge of system disturbances to incorporate into the policy specification procedure, then the choice of model specification will 
become crucial. Given the present state of knowledge concerning what is the 'right' specification of a particular model, it appears that the above problem may not be adequately overcome in a real world application. One could only hope for a solution similar to that obtained for $M$ and $M B$. Perhaps the ideal situation would be to have lagged dependent variables with small coefficients to avoid the strong dependence found in this and the preceding chapter. Such a situation cannot be guaranteed without 'rigging' the data and problem to give the desired results. The resulting structural estimation would of course then be worthless.

The results indicate that, given the model and given adequate adjustment to additive uncertainty, policy planners should choose the supply of money as the appropriate monetary target to aim for and then only if fiscal policy, monetary policy and the rate of interest are allowed to adjust in a completely free manner. That is, fiscal policy is specifically directed towards achieving the monetary target as opposed to being specifically directed towards stabilising the income sector. The choice between the supply of money and the monetary base as the appropriate target rests mainly on the behaviour of the rate of interest as the trade-off between $\mathrm{MB}$ and $\mathrm{RL}$ is very pronounced. The conclusion reached here is opposed to the conclusion obtained by $P R$ who found that the use of a monetary model with an exogenous income sector led to the choice of the rate of interest as the appropriate target. While certain institutional factors may partially account for the difference in the results, it is important to bear in mind that the experiments discussed in this chapter show quite clearly that in the case of the money supply and the rate of interest, the feedback into and from the income and open sector in relation to the money sector is quite important - a factor which has not 
been considered in PR's analysis. The results obtained here cannot be regarded as the final conclusive evidence on the choice of monetary target. The final selection will clearly depend on the richness of the model and the particular targets towards which we attempt to steer the system. At least the results obtained here concur with a portion of the very limited amount of empirical work which has been carried out in the above framework. For example, Shaprio and Holbrook (1970) also concluded that the supply of money was the appropriate monetary target to aim for. The model employed in that study was even more simple than the model used here, but nonetheless illustrated the importance of specifying an interaction between sectors of the economy. A further result of some interest and importance is the reversal in the direction of fiscal policy required when we switch from the money supply target to the rate of interest target and at the same time maintaining an effectively stabilised income sector. In the case of the money supply we can specifically direct fiscal policy towards the monetary target and maintain income stability while in the interest rate case to achieve income stability, fiscal policy must be directed away from the monetary target; a result that is not intuitively clear.

As we have seen, the pegging of the interest rate as opposed to the money supply may be destabilising or even impossible in an open growing economy. A more appropriate interest rate target to aim for may be one that is growing over time which would perhaps allow the target to be achieved without resorting to the severe policy actions set out in this chapter. If the achievement of proximate monetary targets, given various instrument weightings, is likely to produce instability in other sectors then doubt must be placed on the usefulness of aiming for proximate targets in the first place; unless of course policy planners 
feel that they can exert greater control over monetary variables than say income variables due to a lack of information for example (control over monetary variables implies excellent knowledge of the open sector which in itself is a doubtful proposition). Nonetheless, we have seen that a reasonably acceptable result can occur, although perhaps not as successful as being able to aim directly for the achievement of ultimate targets.

The results of the experiments presented in this chapter (and the previous chapter) have a much wider application than merely to the optimal choice of proximate monetary targets. They also illustrate a graphic divergence between the theory of optimal macro stabilisation and the application of this theory to empirically estimated models. The theoretical procedure, especially in the case of fully stochastic models, is to assume the existence of optimal controls. Analytical results for the optimal control vector are then obtained. This approach makes no distinction, and in fact is unable to make a distinction, between economically viable and economically impossible controls. The actual constraints in a system may mean that a control which can be found to exist may be totally unfeasible such as government spending in M3. On this basis, many theoretical propositions may not hold in an empirical framework. A theorist using a simple analytical model could quite easily conclude that in a non-stochastic world it does not matter which monetary target we choose. The results of this chapter clearly show that this need not be the case. 


\section{STRONGLY-TINBERGEN ANALYSIS}

Having looked at the linear/quadratic approach to stabilisation, the analysis now shifts to the fixed target framework and extends the linear/quadratic approach to a wider variety of target configurations for comparative purposes. A convenient point to begin the analysis is with the strongly-Tinbergen situation of as many instruments as targets and where the satisfaction of the instrument rank condition unifies the target path and target point approaches to stabilisation. Each target can be hit in each time period to result in a target path achievement which of course is constructed from a series of exactly achieved target points. The policy planner has the option of doing nothing for a given period of time and then steering the system instantaneously (within the unit time period) to a target path or a target point with the choice of a target path or target point depending largely on political considerations. The stronglyTinbergen framework would enable a government to pursue a course of action designed to attract one sector of the community but sacrificing another sector of the economy in doing so and then stabilising the economy of the appropriate point in time to achieve maximum political gain. The model presented in this study only contains a fiscal and monetary instrument - but in practice a government has a wide variety of instruments available to it and in most cases it would be possible to achieve the necessary conditions for a strongly-Tinbergen solution - as many instruments as targets. Thus, it would not be unreasonable for a government to pursue a target package consisting of an income, balance of payments position, money supply and unemployment target and given policy existence, a solution will be possible. Indeed, the pursuit of the four targets outlined above would be feasible in terms of the number of instruments from which 
a government is able to choose. The problem at the application level is that while policy may exist, the trade-offs between variables and selected target levels may be so strong that the required policy is not feasible. Consider a simple Phillips curve analysis where a government seeks to achieve price and unemployment targets given two instruments with independent affects on both targets. Low target levels: for both variables may be able to be achieved but perhaps only with explosive, negative or other undesirable features in the instruments. Indeed, to obtain a feasible instrument package it may be necessary to specify politically or socially unacceptable target levels (5\% inflation and $10 \%$ unemployment for example) and as such the existence of a strongly-Tinbergen situation should not be viewed as a panacea in relation to the achievement of an economy exactly stabilised at a desirable level.

The limited amount of strongly-Tinbergen analysis already considered in this study illustrates to some extent the problem outlined in the preceding paragraph. In particular the exact achievement of internal and external balance (IEB2) illustrates the degree of adjustment in the monetary instrument required to achieve that target mix. The structure of the model and the feedback between the income, open and monetary sectors contributed to the behaviour of the controls and indeed the same general type of behaviour was found to exist with a wide variety of target specifications for both $Y$ and FR. Similarly, the monetary analysis of chapter six illustrated that both a money supply and rate of interest target could be simultaneously achieved in a strongly-Tinbergen framework but only with disastrous results for the controls. It is safe to say that most economists would not specify the money supply and rate of interest as simultaneous targets due to the inherent trade-off between those variables established by conventional theory. However, many economists would also argue that 
the money supply and the rate of interest would not be compatible with policy existence. This need not be the case as many models, particularly those with an endogenous money supply will yield existence and the fundamental problem is really the inherent structural relationship of the model giving the undesirable trade-offs. Of course, a failure of policy existence with as many instruments as targets is the extreme or polar case of variable trade-offs. For example, a situation in which the achievement of one variable automatically fixes the level of the other target with no possibility of independent instrument manipulation. This proposition will become clearer within the context of an examination of the stronglyTinbergen properties of the model.

Recall that the Tinbergen properties of a model can be simply determined by an analysis of the reduced form (impact) instrument multipliers. A rank analysis for the model of Chapter Three is given in Table 18. Note. that with fifteen exogenous variables and two instruments we can obtain 105 sets of target configurations where the number of targets equals the number of instruments. Many of these lack any real interest from an economic point of view (why aim for a Total Personal Income target and a Disposable Income Target in the absence of tax adjustments) and many others are not compatible with policy existence. Specifically; only endogenous variables of major concern are considered in detail - Y, FR, M and RL. The results indicate a large number of rank failures. The reason for a majority of these rank failures lies not with the value of the structural parameters but with the specification of the behavioural equations and identities. For example, consider targets of $\mathrm{Y}$ and imports, IM. The coupling of these two targets results in an instrument rank failure directly as a result of the appearance of only one endogenous variable in the import function (Y). The remaining variables are either exogenous or lagged endogenous. Thus, the achievement 
of a level of $\mathrm{Y}$ fixes the level of IM and the two cannot be achieved independently. This is an obvious example of an extreme or polar trade-off between variables. In many cases the structure will be more complex and the reason for the rank failure will not be as obvious. While the behavioural relationships for particular variables in the model are very simple and lead directly to rank failures in many cases, larger and more complex models can, and do, suffer from a similar constraint. This is particularly so for short-run models which contain many lagged endogenous variables within particular behavioural relationships thus precluding the achievement of particular combinations of targets and perhaps severely restricting the options of authorities. Of course, such an analysis would depend upon the correct specification of the model which gives rise to the important potential consequence of a government being advised that its targets cannot be achieved, when in fact they can. Friedman would argue that this type of outcome justifies the use of "rules".

The assessment of the instrument rank properties specified in Table 18 was made on the basis of a quantitative analysis of the impact multipliers and an inspection of the structure. Pure reliance on the numerical values of the appropriate multipliers can be misleading due to problems of rounding either by the researcher, the computer or in the format of the output obtained from the computer. The multipliers listed in Table 18 are given to four decimal places and it would appear at first sight that this is sufficient. Consider RL and RS. A rank analysis based on four decimal places gives a rank of two (the determinant is exceptionally low however) which is clearly incorrect as it can be seen from the structural equations that the targets are not independent. It is a relatively easy matter to detect the error when only a small model is involved but it could be extremely difficult to detect such an error in a large complex model. 


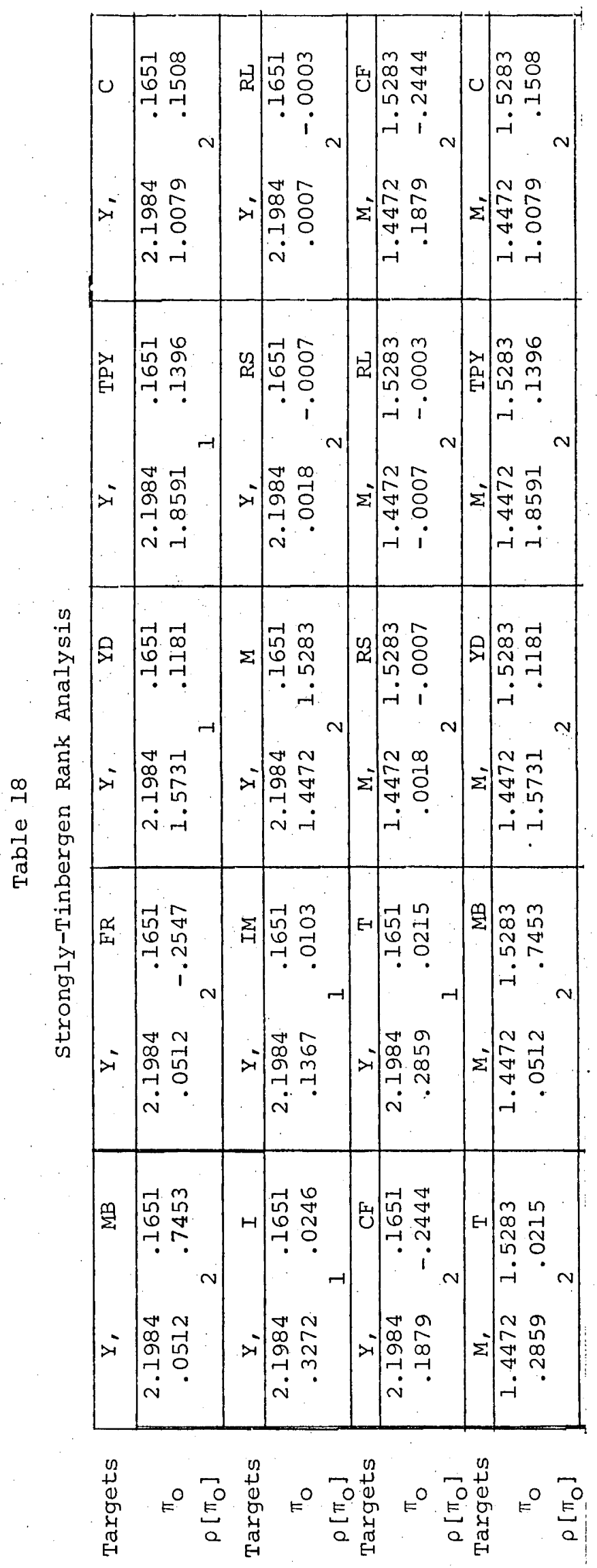

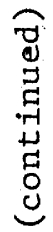




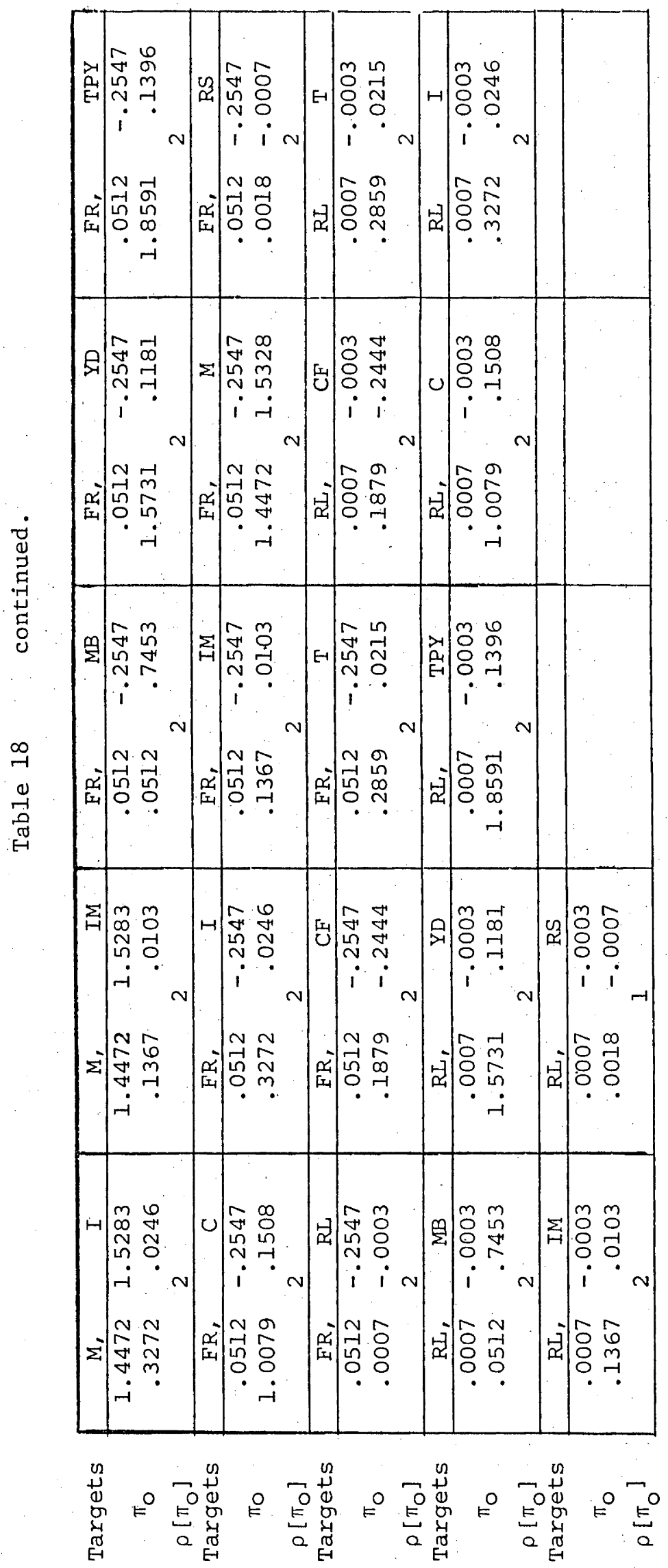


At another level, rounding could produce rank failures in a situation where the targets and instruments of concern are linearly independent, resulting in the abandonment of an otherwise feasible target set. The problems caused by rounding are potentially quite severe and are particularly important in a target path analysis with more targets than instruments as. we shall see below. The small magnitude of many instrument multipliers adds to the problems of rounding and also poses an additional problem. The low values of particular multipliers result in very low values for determinants which can make matrix inversions questionable, particularly if rounding has produced near linear dependence between instruments. The problem is not as severe in a strongly-Tinbergen framework in the case of two or three instruments but once again can be quite severe in the target path framework where the number of time indexed instruments may be as large as forty or fifty with a matrix to be inverted of dimension forty or fifty.

Having looked at some of the problems associated with an applied strongly-Tinbergen analysis we now turn briefly to an analysis within the context of the model. The target pairs included in each experiment should be viewed as natural extensions to the internal and external balance and monetary experiments of previous chapters although a less detailed analysis will be presented here. The target groupings and the general feasibility results are presented in Table 19. The results of Table 18 are used to establish existence. The experiments in this chapter are identified by the prefix FT followed by a numeral indicating the sequence of the experiment. The designation of feasible or infeasible is made on the basis of the required policy mix and or the behaviour of the remainder of the non-target endogenous variable. Note that the target groupings which specify an income target and one monetary or open target are all feasible while groupings of monetary targets and open targets are with one exception, 
Table 19

Strongly-Tinbergen Target Pairs

\begin{tabular}{lllll} 
& & & Feasible & Infeasible \\
FT1 & Y & FR & $\checkmark$ & $\checkmark$ \\
FT2 & Y & M & $\checkmark$ & \\
FT3 & Y & MB & $\checkmark$ & $\checkmark$ \\
FT4 & M & RL & & \\
FT5 & M & FR & $\checkmark$ & $\checkmark$ \\
FT6 & MB & RL & & $\checkmark$ \\
FT7 & Y & CF & $\checkmark$ & $\checkmark$ \\
FT8 & FR & CF & & $\checkmark$ \\
FT9 & FR & RL & & $\checkmark$ \\
FT10 & RL & CF & $\ddots$ & $\checkmark$ \\
\hline
\end{tabular}

infeasible. In each infeasible case the controls took on negative values or fluctuated to such an extent that the resulting time path could not be regarded as a feasible policy option. Stochastic experiments were also performed but the general results remained unchanged under uncertainty. A detailed analysis of the results of the feasible solutions is largely redundant as identical or similar results have already been obtained. For example, FTI is identical to IEB2 and FT2 and FT3 are similar to the monetary experiments in the previous chapter where G was fixed (and Y tracked close to its target), with DM allowed to adjust. In this case both controls are perfectly free to adjust but the inclusion of $\mathrm{Y}$ as a target produces a policy assignment in which $G$ is predominantly directed towards $\mathrm{Y}$ thus giving the same indicative behaviour (but stronger in relation to desired $G$ ) as the optimal monetary experiments. Recall that in particular monetary experiments $G$ was restricted and $\mathrm{Y}$ tracked 
reasonably close to its target. In many cases the gap between the target level and the optimal $\mathrm{Y}$ was very small. The results of this chapter illustrate that to close the gap between actual and desired levels requires what appears to be a proportionately larger adjustment in $G$ in spite of the fact that the impact multiplier for $G$ in relation to $Y$ is relatively large. For example, see figures 28 and 29. This type of behaviour was found in the internal and external balance experiments when the system was shifted from attempted exact stabilisation (IEBI) to a position of exact fine tuning in IEB2. The overall results show that in terms of the model, exact fine tuning requires strong and sometimes excessive adjustment in both fiscal and monetary policy when in fact a high degree of stabilisation can be obtained by sacrificing exact fine tuning (IEBl for example) and opting for less adjustment in the mix of policy. This would be of particular importance if the parameters of the system are likely to adjust through the private sectors response to the government's policy actions. A less active policy package may result in less parameter response and a less volatile adjustment in the private sectors expectations. The problem of excessive instrument adjustment when exact fine tuning is required suggests that a similar and pexhaps more severe result will occur in a non stronglyTinbergen fixed target framework where perhaps four or five targets can be. exactly achieved by varying only two instruments, rather than just hitting two targets as we have done here. Related to this issue is the fact that a large number of target pairs are not consistent with feasible policies and the coupling of these targets with feasible pairs may result in the total target package being unattainable in relation to negative or other infeasible policy levels. On the other hand, it would be desirable for the introduction of policy anticipation to result in the feasible pursuit of targets which are unattainable in a strongly-Tinbergen framework. 


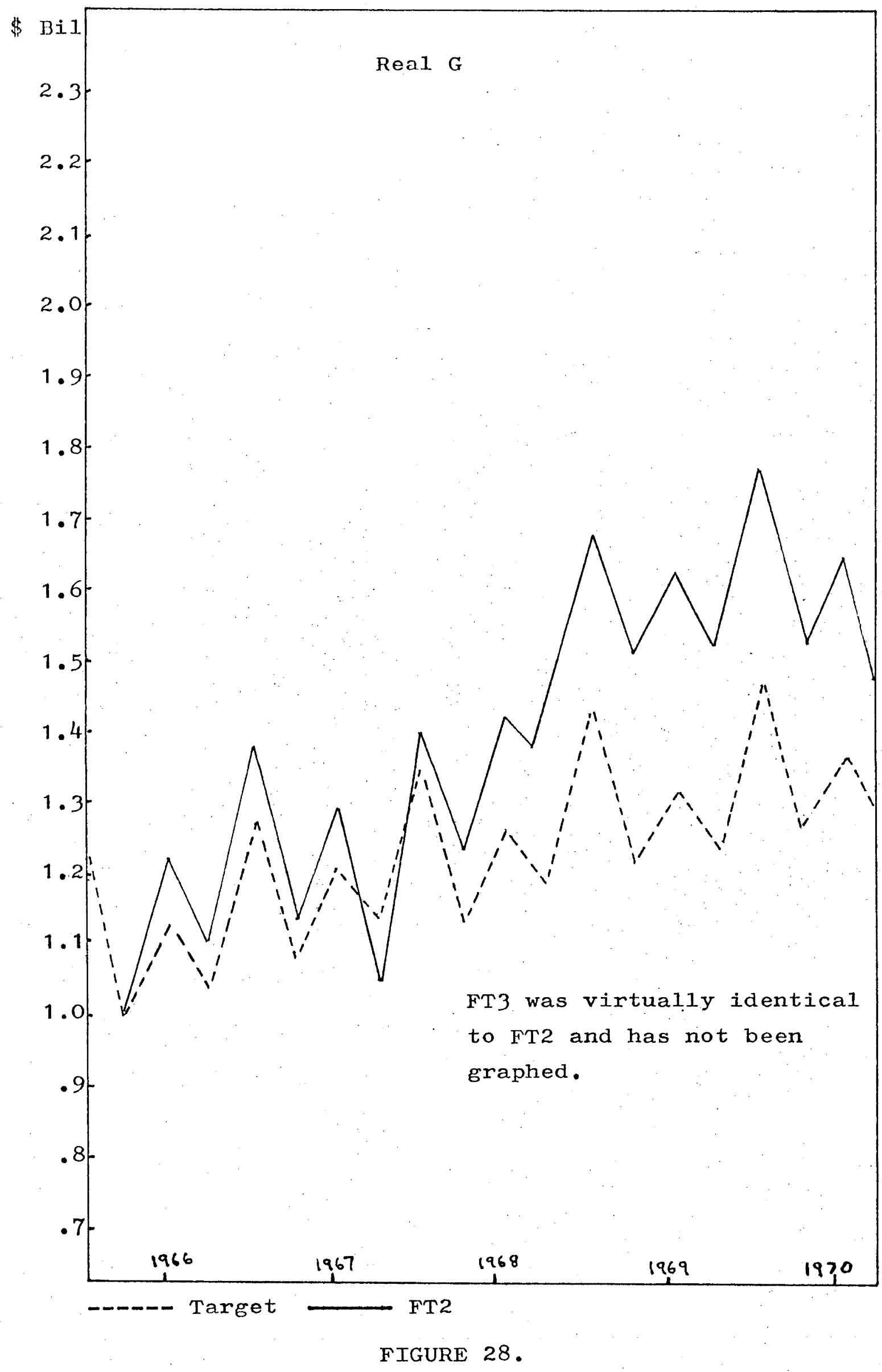




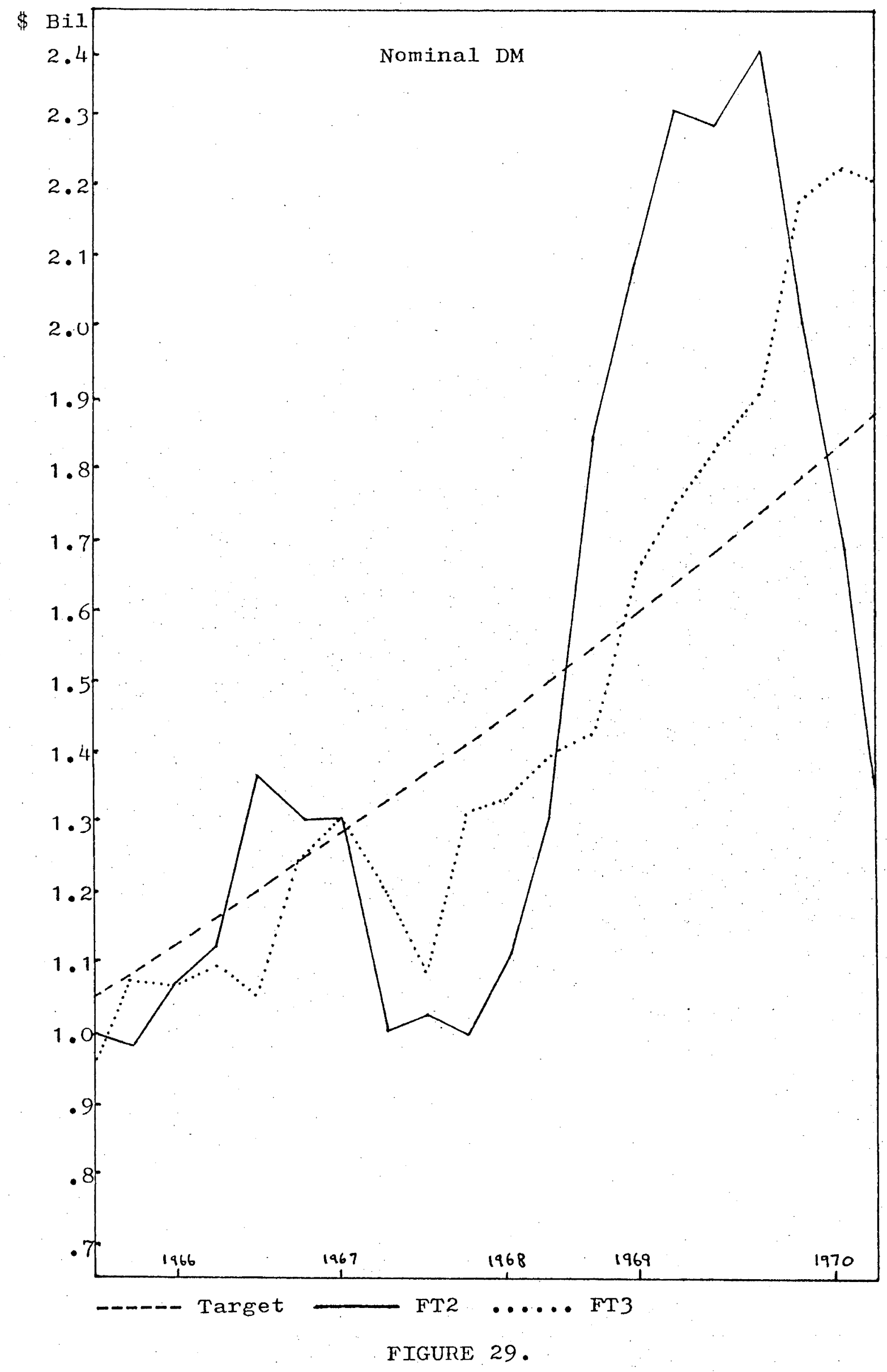


Unfortunately, the results so far suggest that the policy lead target path problem may produce undesirable results given the range of independent targets available. This question will be pursued in some detail in the remainder of this chapter.

Before turning to a target path and target point analysis it is appropriate to briefly comment on a further area of comparison between the linear/quadratic and fixed target approaches. Recall that in a stronglyTinbergen framework, the initial conditions are incorporated in the solution procedure. Not so however, in an optimisation framework where the control laws are computed independently of the initial and subsequent states of the system. Thus it could be expected that in an optimising framework with as many independent targets as instruments, some settling down of the system may occur as the system adjusts to the initial conditions. The result being that the targets may not be exactly hit until after the planning period has commenced (assuming no stochastic disturbances). The detailed discussion of IEB2 in chapter five illustrated that only a negligible difference occurred between the optimisation and fixed target solutions. This result followed directly from the relationship between the target values of $\mathrm{Y}$ and $\mathrm{FR}$ and the appropriate initial conditions and from the presence of $\mathrm{Y}_{\mathrm{t}-1}$ and $\mathrm{FR}_{\mathrm{t}-1}$ as significant components of the feedback matrices. A similar comparison between experiments FT2 to FT10 with corresponding linear/quadratic solutions was made with the result that in most linear/quadratic solutions some settling down was required. This tendency was possibly reinforced by the non-appearance of $\mathrm{M}, \mathrm{MB}$ and $\mathrm{CR}$ in the relevant feedback matrices. In most cases the divergence between optimal path and target path was slight and in all cases the target variable of concern was exactly on target by the mid. point of the planning period. Thus, the two techniques are largely interchangeable with either technique giving the same general indicative 
results. If the targets were extremely divergent from the initial conditions then the linear/quadratic solution could become substantially inferior to the fixed target solution.

\section{TARGET PATH AND TARGET POINT ANTICIPATION}

The dynamic analogue to Tinbergen's famous counting rule can be used to establish a priori the dimensions of the required controllability matrix and necessary lead for given numbers of targets and instruments. The counting is given by

$$
" k "=k(s+T+1) \geq " m " \equiv m(T+1)
$$

where it will be recalled from Chapter Two that " $k$ " and " $m$ " represent the number of time indexed controls and targets respectively, $s$ is the policy lead, $\mathrm{T}+\mathrm{l}$ is the target path and consequently $\mathrm{k}+\mathrm{T}+\mathrm{l}$ equals the planning period. The choice of $\mathrm{s}$ and $\mathrm{T}+\mathrm{l}$ given the targets and instruments rests crucially on the length of the planning period. To maintain consistency with the linear/quadratic experiments the policy horizon will also be set equal to twenty periods. Given two instruments we are then able to establish some properties of the fixed target experiments. Firstly consider four targets. From the necessary conditions it is immediately established that the number of time indexed instruments is equal to the number of "true" and "natural" instruments times the length of the policy horizon which equals 40 in this case. It then remains to determine the length of $\mathrm{s}$ and $\mathrm{T}+\mathrm{I}$. which will then give us " $m$ ". The establishment of $s$ and $T+1$ can be pursued by a process of elimination by specifying various policy leads until the necessary conditions are met. A simpler procedure is to simply multiply the 
number of targets by specific numbers (less than the length of the planning period) until equality with " $k$ " is established or the closest integer to it is obtained. This will then give $\mathrm{T}+\mathrm{l}$ fxom which the value of $\mathrm{s}$ follows immediately. The process is exceptionally simple in the case of two instruments and four targets: "k" $=40$ and it is obvious from the necessary conditions that $\mathrm{T}+1$ must equal 10. Thus $\mathrm{s}=10$. This implies that provided the controllability matrix for specific targets has full rank $(\rho[\hat{R}]=40)$ then the entire four targets can be exactly achieved provided the policy planner begins to manipulate his instruments ten periods in advance. Now consider three targets. Once again the number of time indexed instruments equals 40 . With three targets the closest integer less than 40 we can obtain will be $3(T+1)=3 \times 13=39$. Thus equality in the necessary conditions will not hold. The dropping of one target now allows the policy planner to hit his targets for three additional periods with a policy lead of only seven periods. However, as " $m$ " and " $k$ " are not equal we will be unable to solve for " $m$ " unless the controllability matrix of dimension " $k$ " $x$ " $m$ " is adjusted such that it is of order " $m$ " [and full rank is obtained]. In our example, this can be achieved by dropping one time indexed instrument as clearly we have a surfeit of instruments. The deleted or slack instrument can then be assigned a value a priori, treated as given and then included with the uncontrollable but known exogenous variables. The choice of a suitable "instrument" to delete can be quite crucial and we shall consider this question below. The above analysis of the necessary. conditions illustrates some important factors associated with fixed target solutions. Firstly, the more targets we have relative to natural instruments the smaller in length will be the required target path and the longer will be the necessary policy lead given a specific policy horizon. As the analysis assumes a fixed structure and fixed structural parameters, the 
extension of a planning period to obtain a greater degree of exact fine tuning could result in destabilisation, particularly if the structure shifts, as it is likely to do, during the process of policy implementation. This problem is also faced in the linear/quadratic framework but in that case the problem can be minimised by reducing the length of the policy horizon and updating our knowledge of the system structure. A reduction in the length of the policy horizon in the fixed target framework will result in the abandonment of our original target path objective and of course, given our present state of knowledge, there is no provision for updating our knowledge of the system structure through a learning process given the nature of the fixed target solution procedure. Returning briefly to the strongly-Tinbergen framework we can see that only in this special case is the specification of a specific planning horizon largely irrelevant and a learning process is feasible as decisions are made from period to period. Secondly, the more targets we have relative to natural instruments and the longer we require our target path to be, the more difficult computation becomes as the dimensions of the controllability matrix will increase dramatically making inversion extremely difficult, if not impossible. This will become apparent below as particular solutions are considered. For this reason and because the preceding analysis has established that not all endogenous variables represent independent targets and some of those that are cannot be combined in an economically meaningful fashion, the maximum number of natural targets considered in this portion of the study will be 4. The policy lead and target path requirements for this situation have already been established and should be stressed again that the dimensions of the controllability matrix will be $40 \times 40$ - a matrix which could be difficult to invert. Ideally, we would like to have at our disposal as many instruments as possible as this would 
allow us to hit more targets with the same policy lead or extend the length of the target path with a considerably reduced policy lead for the same number of targets. As yet no mention has been made of the necessary conditions for target point problems. The counting rule requirements for target point problems are relatively simple and the matrix inverted will always be equal to the number of natural targets. A discussion will be developed in conjunction with the solution presented below. Having discussed and established the necessary conditions for the fixed target study of this chapter, we can establish one other property related to the model before proceeding to a discussion of sufficiency for particular target configurations. Recall from chapter two that the CayleyHamilton Theorem placed an upper bound on the pursuit of varying lengths in the target path by specifying a maximum policy lead after which no additional linearly independent time indexed instruments could be obtained. In relation to the state space version of the model this constraint confines the analysis to a policy horizon of 52 periods and $s=26$ (assuming four targets) and allows considerable scope for the extension of the target path over and above that obtainable with a twenty period horizon. However, the solution of the problem with a 26 period lead would be prohibitive as it would require the inversion of a matrix of order 104. This may be possible if the elements of the matrix are all non-zero and greater than plus or minus one but would most likely not be possible in the context employed here due to the structure of the controllability matrix and the tendency for a majority of the dynamic multipliers to disappear to zero. Once again this will become apparent when various target packages are analysed below. It is also highly unlikely that the pursuit of a prolonged policy strategy over 52 periods would be economically feasible in a quarterly model and would certainly be impossible in an annual model although a monthly or weekly 
model would possibly be feasible. The use of a monthly model such as the monetary model employed by Pindyck and Roberts [1964] [1976] would perhaps allow for the maximum degree of target path achievement until constrained by the limits set by the Cayley-Hamilton Theorem. The upper bound placed upon the length of policy lead does allow in theory for considerable scope in the variation of the length of the target path but computational realities make it somewhat prohibitive. As such, no attempt will be made here to achieve the maximum degree of anticipation and target path achievement. Indeed, an analysis over twenty periods is very close to being computationally prohibitive.

The next stage in the solution procedure is to compute the dynamic instrument and exogenous variable multipliers which are required in the solution procedures of both target path and target point problems. The dynamic multipliers can be computed using any state space realisation provided of course that the technique is consistent with the state space realisation. As a variant of the Chow-Turnovsky realisation has been used, Equation (4.17) of Chapter Four will be used. The resulting dynamic instrument multipliers for each endogenous variable are listed in Tables 20 and 21 with a summary of the cumulative multipliers given in Table 22 . As could be expected, the majority of multipliers for particular variables fluctuate between positive and negative values. Note that the numerical values of the multipliers are very small, particularly in the case of the rates of interest. As the target path controllability matrices will consist of a large number of zeros a priori, the presence of non-zero elements which are close to zero could result in inversion difficulties. The multipliers also give some indication of the stability properties of the system referred to in the instrument instability analysis of Chapter Four. Bearing in mind that the multipliers represent the effect over time of 


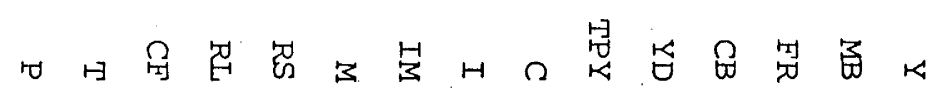

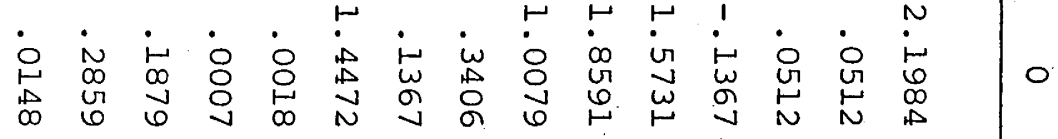

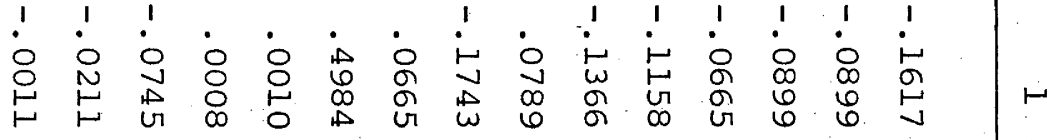

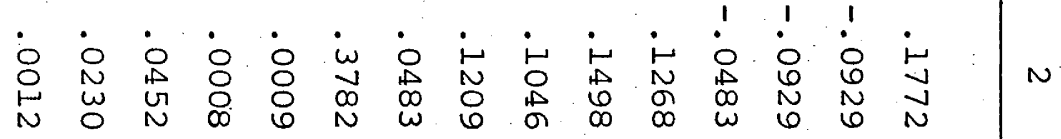

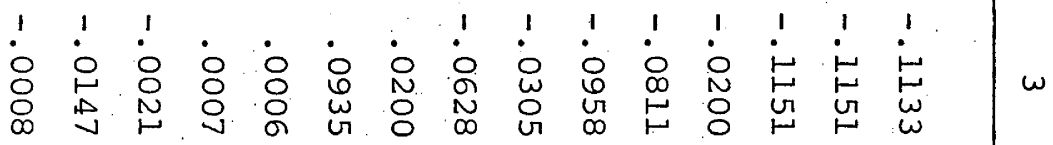

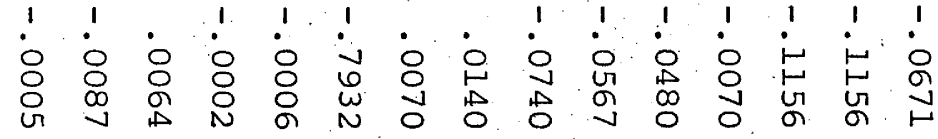

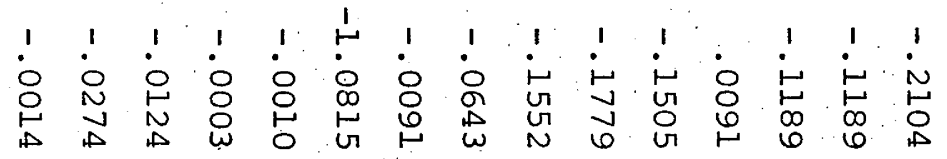

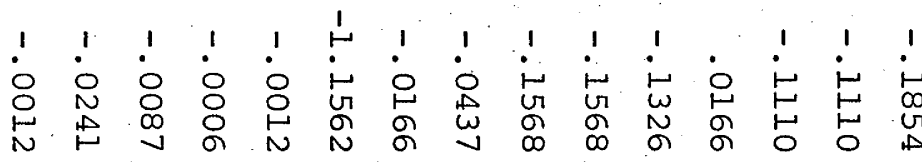

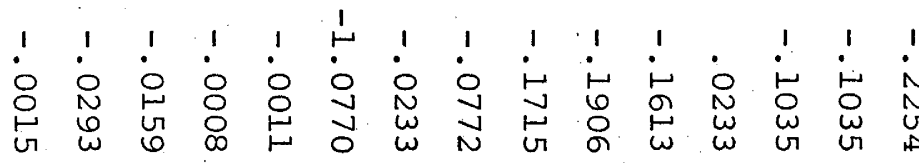

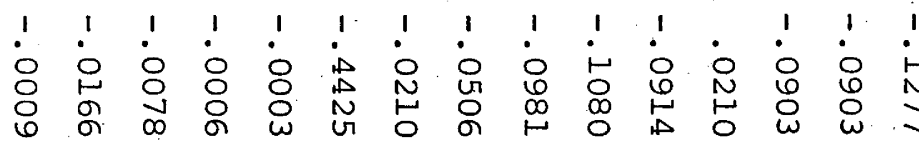

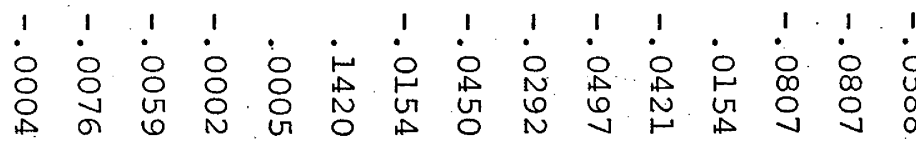

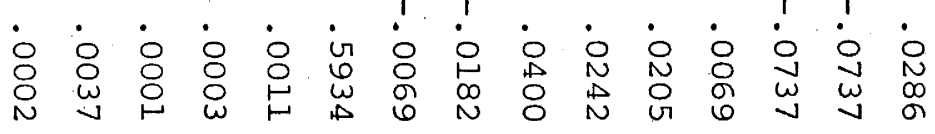




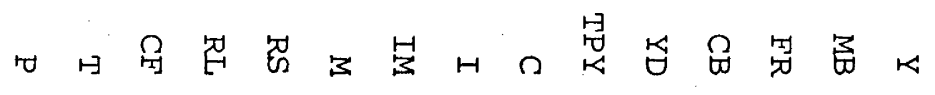

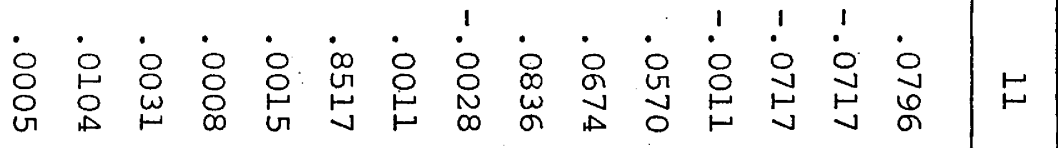

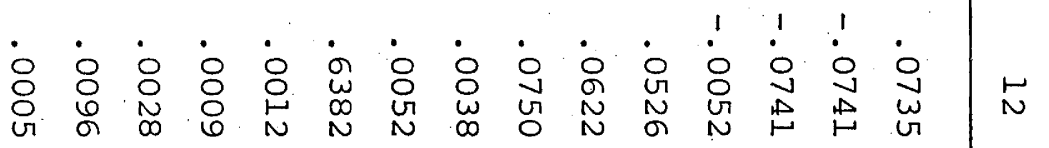

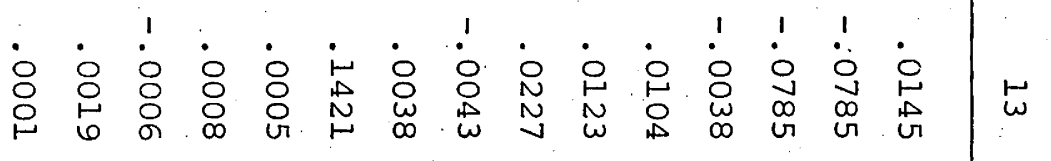

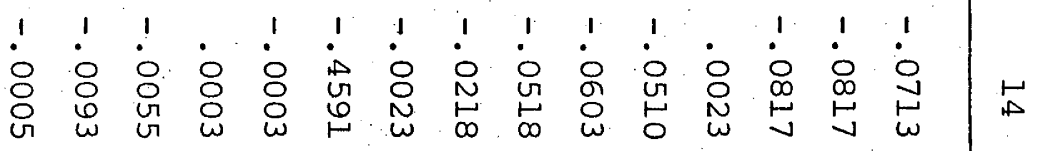

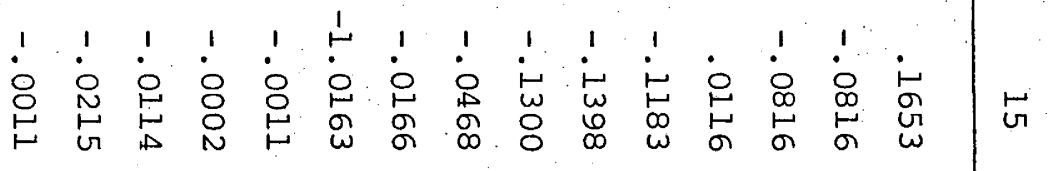

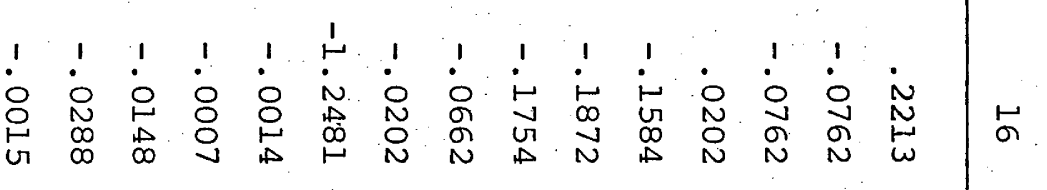

点

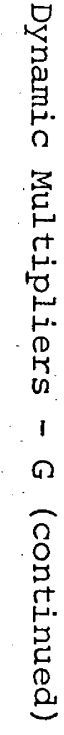

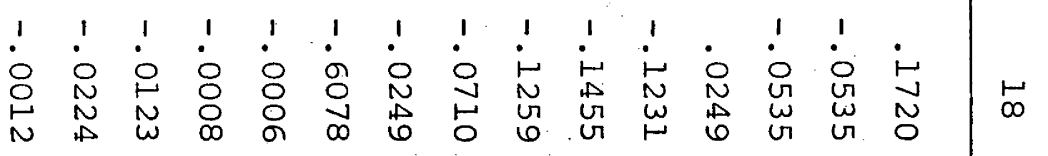

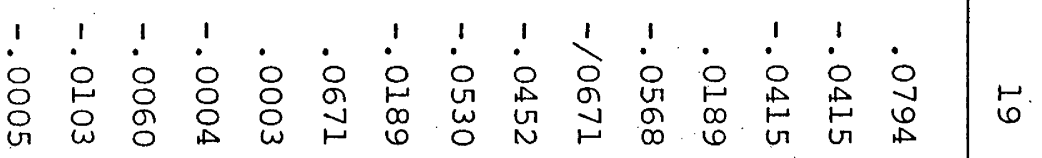

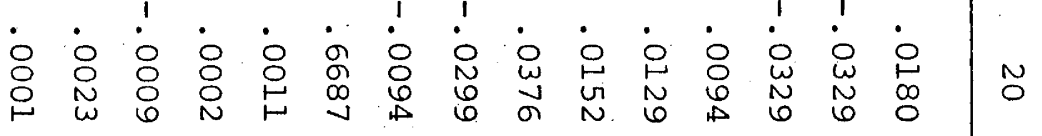




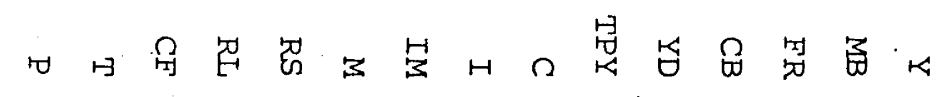

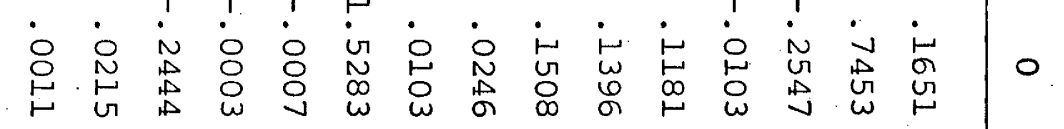

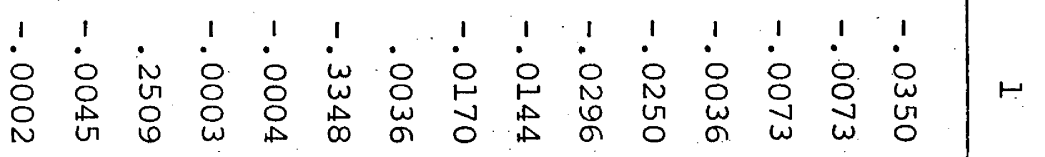

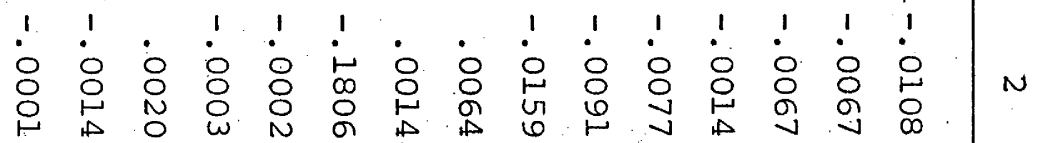

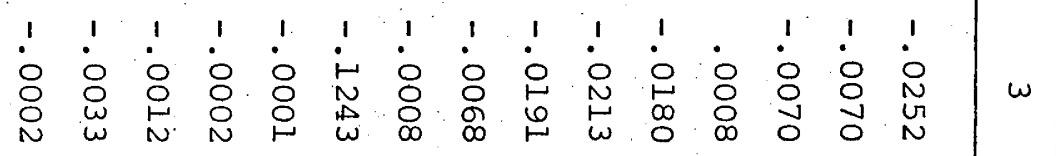

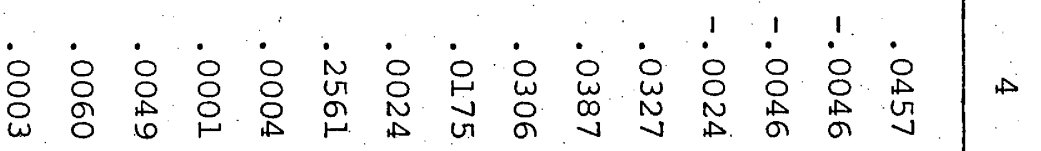

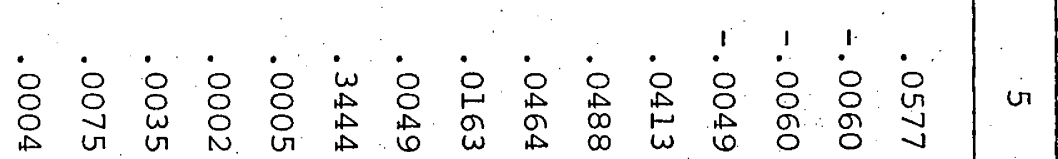

暿

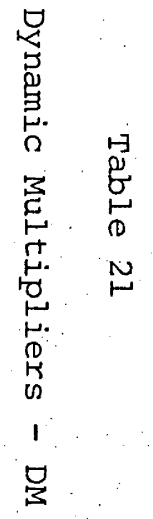

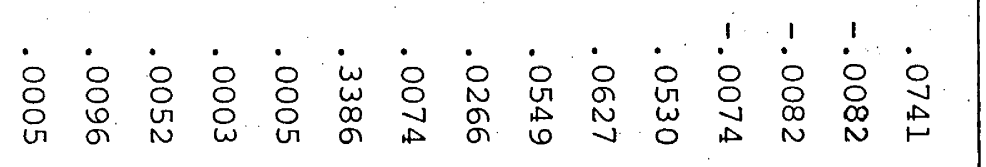

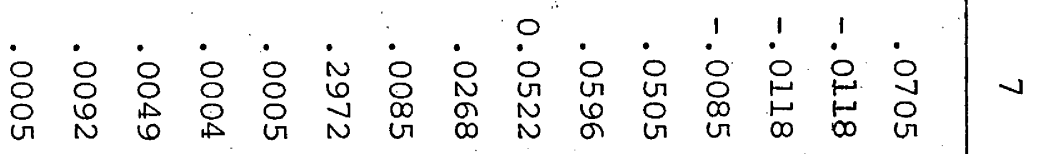

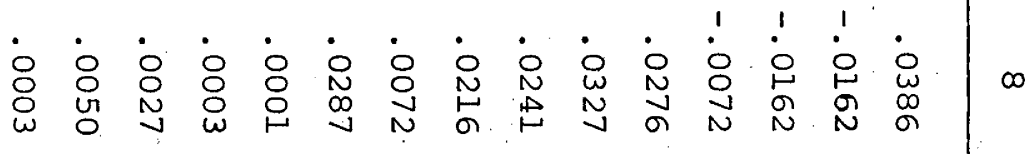

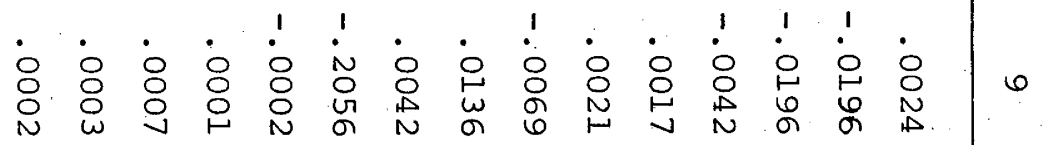

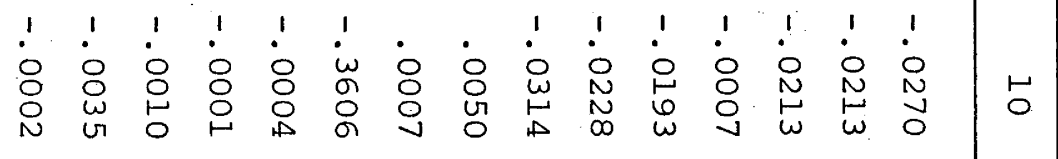




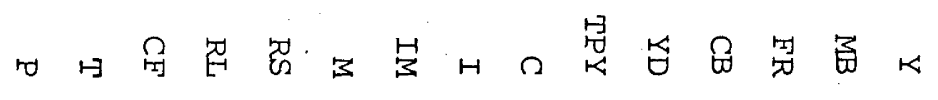

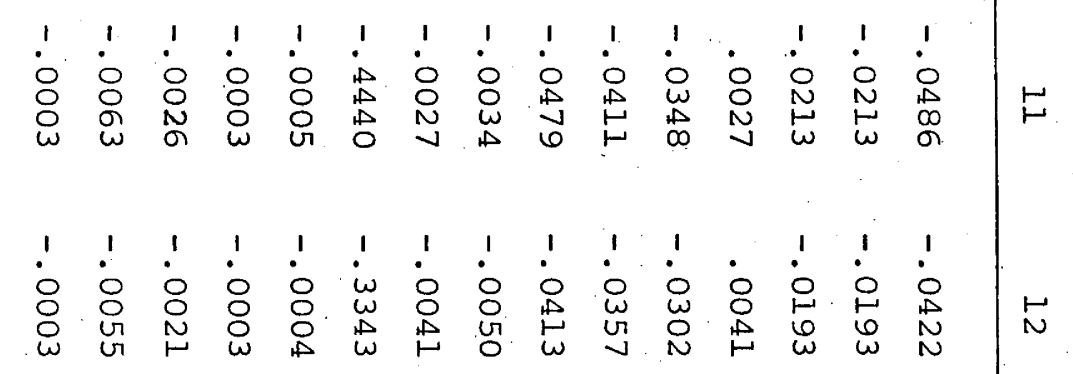

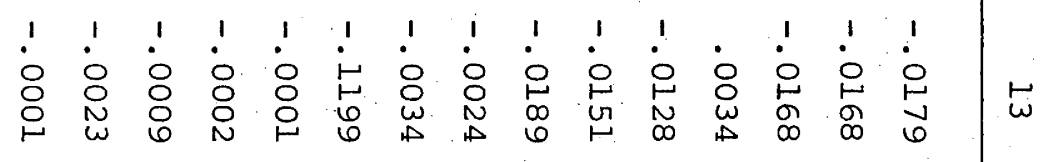

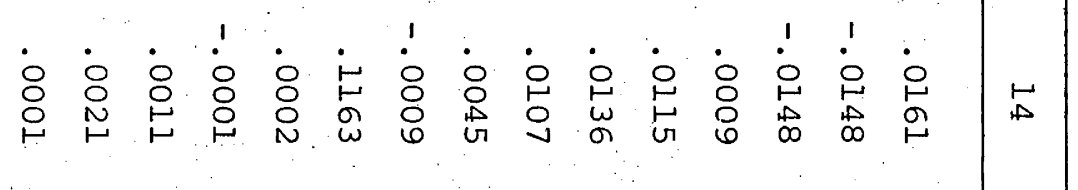

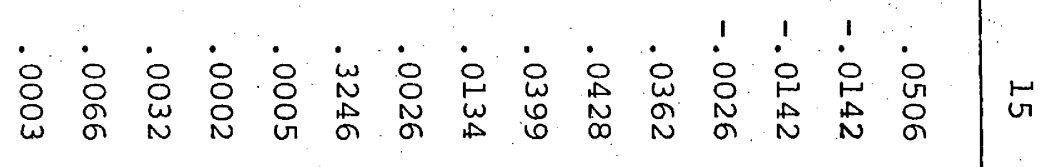

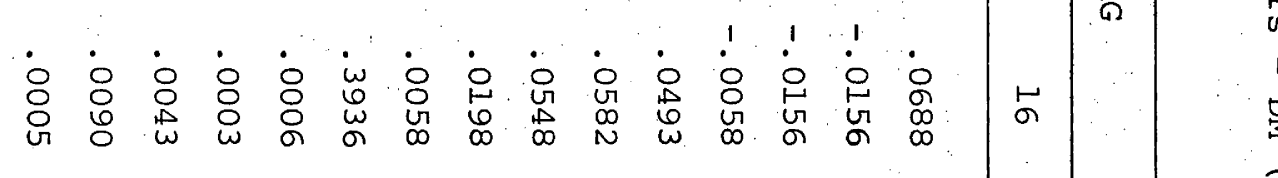

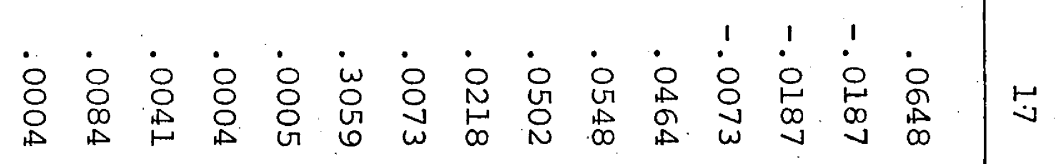

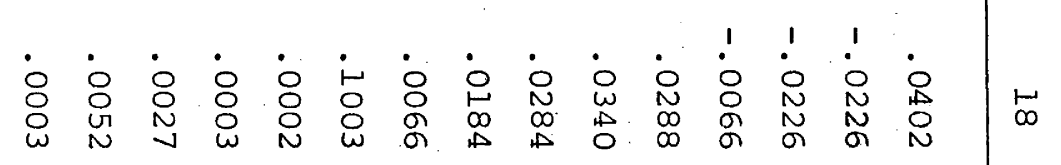

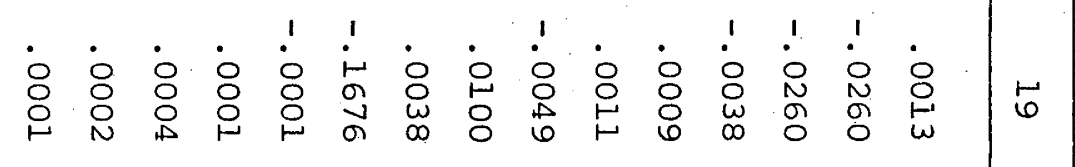

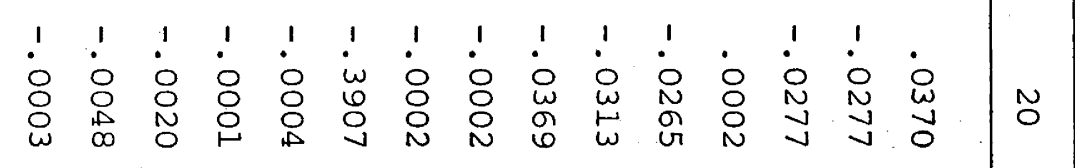




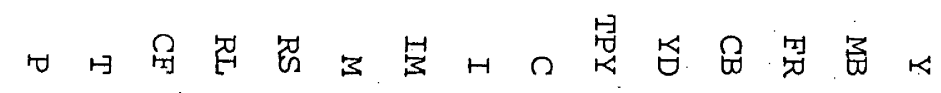

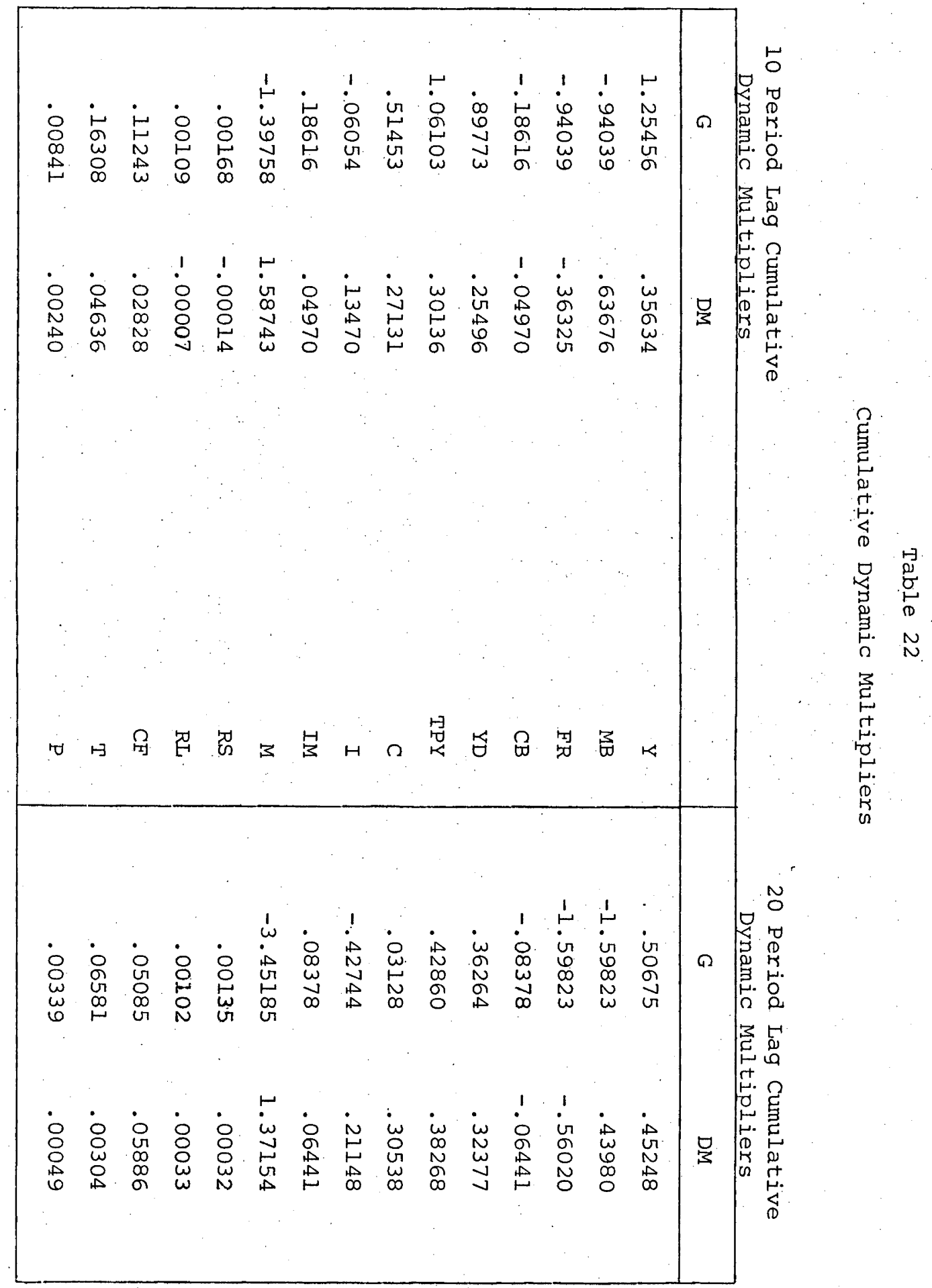


a unit impulse in a particular instrument, if the cumulative effect of the impulse tends to increase over time then it will indicate a degree of instability in the endogenous variable of concern. If the multipliers clearly blow-up over time then this will certainly indicate the presence of severe instability. Conversely, if the cumulative effect declines and the multipliers tend to zero then this will indicate stability in the particular endogenous variable referred to. An inspection of the multipliers suggests that a degree of instability could exist in the open and monetary sectors. The cumulative multipliers support this, particularly in relation to $\mathrm{FR}$ and $\mathrm{MB}$. This tendency is of course a direct result of the presence of FR in the model, and the fact that the identity for FR contains $\mathrm{FR}_{\mathrm{t}-1}$. If $\mathrm{FR}_{\mathrm{t}-1}$ and $\mathrm{FR}$ begins to expand rapidly without any offsetting impact from monetary and fiscal policy then $M B$ and $M$ will react accordingly. In any case, the tendency for a unit policy impulse to have an increasing effect does not appear to be particularly serious. Indeed, the tendency for particular multipliers to increase over time will be of some assistance in the computation of the required instrument vector as it will eliminate near zero elements which could be cause for concern in a large matrix. The tendency for particular multipliers to increase over time is not characteristic of both instruments. For example, fiscal policy has a reasonably rapid declining influence on income and consumption. On the other hand monetary policy has a marginally increasing cumulative effect on $\mathrm{Y}$ and on $\mathrm{C}$ due to the influence of $\mathrm{M}$ and $\mathrm{FR}$. Fortunately, the low value of the wealth effect in the consumption function dampens the monetary effect. The tendency for multipliers to increase or decrease over time will of course affect the nature of the results. Finally, the instrument multipliers provide a graphic example of how at the impact level, a set of targets may be subject to linearly independent instrument effects but subsequent linear 
dependencies may appear in the lagged multipliers. An obvious example of this are the multipliers for $\mathrm{FR}$ and $\mathrm{MB}$. The fiscal multipliers differ at the impact level but due to the lag and. system structure of the model, become identical after one time period has elapsed creating strict linear dependence with the fiscal instrument. This effect is obvious here and the cause can be readily traced back to the structural properties of the model, but in a larger, more complex model it may be difficult to detect, particularly if the multipliers are very small and rounding has occurred.

The purpose of the following experiments was twofold. Firstly, they will provide the first computational experience with target path anticipation problems and as such are valuable in their own right. Secondly, they were intended as an extension of the earlier internal and external balance and monetary experiments, at the same time providing a comparison with linear/quadratic techniques. The experiments consisted of analysing the achievement of firstly four sets of targets and then three sets of targets, beginning with an analysis of policy existence and providing the rank conditions were met, moving on to the actual computation of the policy vector. As with the earlier experiments in this chapter, they are identified by the pre-fix FT. The target variables for each experiment are listed in the following table. Identical target groupings were employed in the target point solutions.

The solution for FTll requires a (40x40) controllability matrix and from the system dimensions given in Chapter Two, the matrix of exogenous variable multipliers will be of dimension $(40 \times 200)$ and the dimension of $\hat{\mathrm{P}}$ will be $(40 \times 26)$. The solution to the problem is formidable in terms of the matrices to be manipulated. The dimensions given above were not restrictive in terms of computation on the Australian National University's Univac 1100 
Table 23

Targets - Target Path Anticipation Problem

\begin{tabular}{|c|c|c|c|c|c|c|}
\hline & $\dot{Y}$ & FR & $\mathrm{C}$ & $\mathrm{CF}$ & M & $\mathrm{RL}$ \\
\hline FTII & $\checkmark$ & $\sqrt{ }$ & $\checkmark$ & $\checkmark$ & & \\
\hline FT12 & y & $\checkmark$ & $\checkmark$ & & & \\
\hline FT13 & $\checkmark$ & $\sqrt{ }$ & & $\checkmark$ & & \\
\hline FTl4 & $\sqrt{ }$ & & $\sqrt{ }$ & $\checkmark$ & & \\
\hline FT15 & $\checkmark$ & $\sqrt{ }$ & & & $\checkmark$ & $\checkmark$ \\
\hline FT16 & $\checkmark$ & $\checkmark$ & & & $\checkmark$ & \\
\hline FT17 & $\sqrt{ }$ & $\sqrt{ }$ & & & & $\checkmark$ \\
\hline FT18 & $\sqrt{ }$ & & $\sqrt{ }$ & & $\checkmark$ & \\
\hline FT19 & $\checkmark$ & & $\checkmark$ & & & $\checkmark$ \\
\hline
\end{tabular}

but it is highly likely that for much larger models the dimensions of the matrices could exceed the available core size available at a particular computer installation. In this case a long-winded but effective technique can be adopted. The matrices of exogenous variable and initial condition multipliers can be partitioned to form smaller matrices. The resulting matrices can then be multiplied with the relevant subset of exogenous variable forecasts or set of initial conditions and stored on tape. Each subset of the matrices can be handled in this way with the final result being obtained by incorporating the results of subsets into one final vector. Usually, the storage of a large vector will not be prohibitive. Unfortunately, little can be done about the controllability matrix $\hat{R}$. It is generally not possible to break it up into partitions which do not interact with the remainder of the matrix thus making it impossible to carry out separate independent inversions. This type of solution would only be possible when sectors of a model are completely unrelated and are 
subject to the actions of controls which do not appear in any other sector. Given this situation, a target problem could be solved as two or more separate problems. The high degree of simultaneity of modern macro models would most likely preclude this from happening. The size and the nature of the magnitude of the dynamic instrument multipliers poses the problem of satisfactorily computing the desired inverse, even when the matrix has full rank. To illustrate the problems associated with $\hat{R}$ and to illustrate how how a particulax controllability matrix is set up, the complete controllability matrix for FTll is presented on the following pages. The original computer output has been presented to avoid any typing errcrs and the elements have been rounded to five decimal places. At first sight it would appear that it would be a formidable task to set up the data for such a matrix to be fed into the computer. once again, an extremely efficient simplifying procedure can be adopted. Recall that the construction of the matrix $\hat{R}$ if of the form

$$
\left[\begin{array}{lllll}
\pi_{0} & \pi_{1} & \pi_{2} & \ldots & \ldots \\
\cdots & \pi_{0} & \pi_{1} & \cdots & \ldots \\
\cdots & \ldots & \pi_{0} & \ldots & \ldots
\end{array}\right]
$$

This structure is evident in the printout of $\hat{R}$ for FTll. The setting of the matrix can then take two distinct paths, depending on the availability of computer core. If the computer is very large and the dimensions of the problem number of targets, number of instruments and the length of the planning period) are not prohibitive then $\hat{R}$ can be formed in the same computational process as the dynamic multipliers and it should be clear that only the instrument multipliers for the number of lags equal to the column dimension are required. Similarly, if the matrix has to be inserted into the computer as data, only the abovementioned multipliers need to be 
included. (7.1) shows that it is a simple matter to construct $\hat{R}$ using a simple algorithmic procedure. The first $k(T+S+I)$ columns are filled along with the first $m$ rows by the insertion of the appropriate $k(T+s+1)$ period point controllability matrix. Next, the computer is directed to insert a $k(T+s+1)-k$ point controllability matrix in rows $m+1$ to $2 \mathrm{~m}$ beginning in column $\mathrm{k}+1$ and ending in column $\mathrm{k}(\mathrm{T}+\mathrm{S}+1)$. The above process is repeated until the complete dimensions of the matrix $\hat{R}$ are fulfilled. The process only requires the manipulation of a much smaller point controllability matrix.

Turning now to $\hat{\mathrm{R}}$ for FTll we can observe a number of problems. Firstly, the first two columns only have positive entries for the first four rows giving 36 zero entries for remainder of the elements. Given the low magnitudes of the multipliers with perhaps the exception of the fiscal impact on $\mathrm{Y}$ and $\mathrm{C}$, the close proximity of other variables to zero could have the effect of producing an inverse of doubtful accuracy. Indeed, the large number of zeros in the matrix could pose severe inversion problems. It is not until the eighteenth column that the zeros disappear. The tendency of a fiscal impulse on $Y, C$ and $C F$ to tend to zero has the effect of producing very small values to appear in the extreme right hand columns of the matrix. Once again this could lead to inversion problems due to the close proximity of the elements to zero. The size of the matrix will amplify this problem during computation due to the need to carry out arithmetic procedures with a large number of small numerals; as a result rounding errors are likely to occur which could easily produce a rank failure. The problem became even more severe when $\mathrm{RL}$ was included as a target. It is clear even from a cursory examination of $\hat{R}$ for FTll that it would be almost impossible to detect a rank failure a priori and it is equally clear that the nature of the elements of $\hat{R}$ could prevent the 


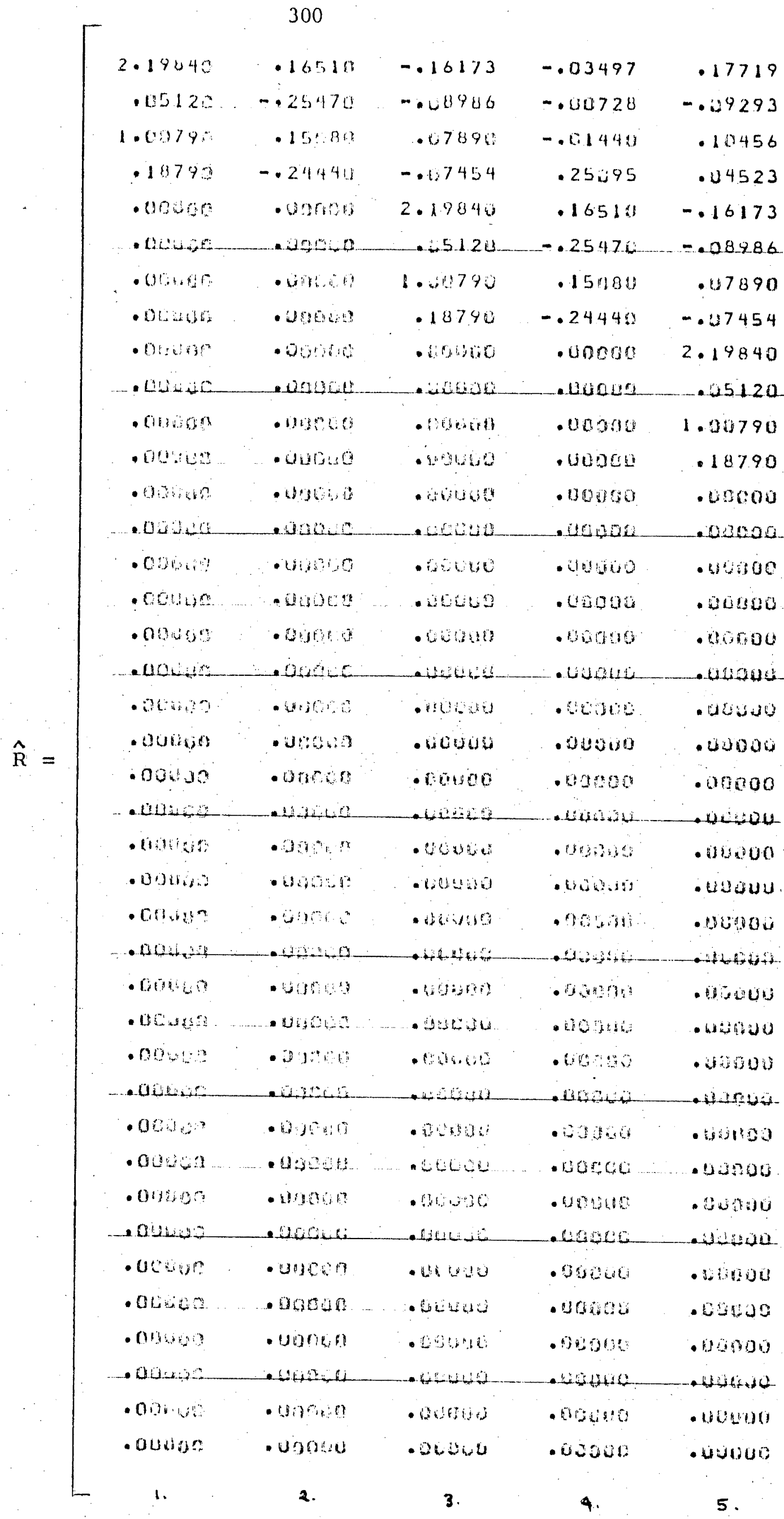




\begin{tabular}{|c|c|c|c|c|}
\hline-0.11 & -.11 .328 & -.52 & -.06719 & $\cdot 0$ \\
\hline-00065 & $-.115-5$ & - $\sin 72$ & -.11565 &.- \\
\hline-.01546 & -03148 & -11914 & -.147405 & .0306 \\
\hline .02198 & -000212 & -14117 & .00644 & .1048 \\
\hline. .103497 & .17719 & $-\ldots i$ & -11328 & -.4251 \\
\hline-.01228 & -19293 & -40065 & -11505 & -.01 \\
\hline $.0144:$ & 10456 & $\cdots 1586$ & -.03048 & -.1191 \\
\hline $.25: 45$ & 0.94523 & .64 & -.05212 & -.0011 \\
\hline .16510 & $-16: 73$ & -.3497 & .17719 & -.0100 \\
\hline-.25420 & $=.18286$ & - & -.09293 & $=.1$ \\
\hline .1568 & .07890 & -0.3144 & .10456 & -.01 \\
\hline .2444 & -.07454 & .25495 & .114523 & .4119 \\
\hline - ondurit & $2.1984 \%$ & .16510 & -.1 & - \\
\hline .0030 & $-2: 5: 20$ & $-.2547 \mathrm{~s}$ & -.1 & - H. H \\
\hline .00408 & $1 \cdot 01799$ & .15080 & .07890 & - \\
\hline . ODELEL & 18 & -.24440 & -.1 & 0 \\
\hline $00418=$ & - $15+100$ & - ockbon & 2.19840 & \\
\hline oostisia & QUOMLL & .tpitisull & .051218 & -.2547 \\
\hline $.00<5$ & - 43 & - actos & 1.00790 & .1 \\
\hline - opuar & to & .60050 & .18790 & -.1 \\
\hline .00503 & - vanas & .40000 & - asingn & - 10000 \\
\hline Dis: & 236 & - UBLULO & - 55aua & - Sicia \\
\hline - $010 \mathrm{sin}$ & - untrit? & - 40000 & & \\
\hline .00 sus & - $\operatorname{sic} 00$ & - GLugle & - 80 ancs & \\
\hline $.0004 ?$ & - Lis?l? & - artuss & - Cuntia & $\theta$ \\
\hline 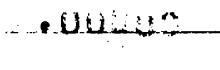 & 2900 & - $\operatorname{coctros}$ & - L & .1 \\
\hline $.00,35$ & - 10000 & - 30400 & 14 & \\
\hline - conting & - 4548 & -42940 & - bastog & .0 \\
\hline - Dristato & - $\cos 20 t$ & $-120 \cos 3$ & andis & \\
\hline - Ouland & . Viser & . . . . n & - LEDILE & .0100 \\
\hline - 1013 & - & & & \\
\hline .03400 & a 4000 & & - vatadias & \\
\hline - Olonary & - Dominget & 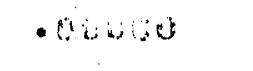 & 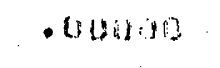 & \\
\hline .011 & 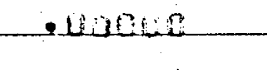 & -6 & $-.000 \mathrm{in}$ & \\
\hline - grais? & - 10048 & - 2000 & .00000 & .0030 \\
\hline - OLisis? & - BDOu & - $4 L U \Delta 0$ & $.00190:$ & - $4:$ \\
\hline - $110,3,0$ & - oundor? & - hougat & - octarso & $.450 u$ \\
\hline & -19020 & - ucugan & .5003 & .0135 \\
\hline - 093264 & - ung:ir? & .00500 & .00270 & -310 \\
\hline 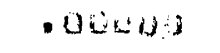 & - Uital S & 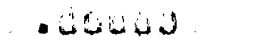 & Bus & \\
\hline
\end{tabular}




\begin{tabular}{|c|c|c|c|c|}
\hline-.21035 & .05773 & -.18536 & .07411 & -.22537 \\
\hline-.11309 & -.04597 & -.11 .97 & -.00815 & -13349 \\
\hline .15522 & .94636 & -.15834 & .015483 & -.17147 \\
\hline-.111238 & - 1352 &.$- \sin 872$ & .00519 & -.01585 \\
\hline$-.0: 673$ & .034575 & $-.21+35$ & .155773 & -.18536 \\
\hline-.11565 &,- 02456 & -.11889 & -.00597 & -.11097 \\
\hline-.8745 & .03063 & -.15522 & .04636 &. .15834 \\
\hline .00644 & .190486 & -.1238 & .09352 & -.00872 \\
\hline-.11328 & -012516 & $-.15671 r$ & .04575 & -.21035 \\
\hline-11505 & -.111722 & -.11565 & -.090456 & -.11889 \\
\hline-01248 & -01914 & -.07455 & .03063 & -.15522 \\
\hline-112212 & -011117 & $\cdot \sin 644$ & - 00486 & -.01238 \\
\hline .17719 & $-41: 8:$ & -11328 & -02516 & -.06710 \\
\hline-09293 & $-.2 \log 6$ & -.11565 & -.00722 & -.11565 \\
\hline .10456 & -01566 & -.03 .148 & -.01914 & $-.10) 7405$ \\
\hline .04523 & .01294 & -10212 & -.00117 & .013644 \\
\hline-.16173 & $-1,3487$ & .177 .9 & -.11080 & -.11328 \\
\hline-08966 & $=.20728$ & -29293 & -0.13665 & -.11505 \\
\hline $.0789 ?$ & -2144.3 & .14456 & -.01586 & -.43448 \\
\hline-007454 & .25395 & .14523 & .00198 & -.110212 \\
\hline 2.19640 & $.1651 \%$ & -.16173 & -.03497 & .17719 \\
\hline .05120 & -.2547 & $=28286$ & $=.10728$ & -.49293 \\
\hline i. $\operatorname{lin}(179)$ & $.153: 83$ & .47890 & -01440 & .10456 \\
\hline .18798 & -.24440 & -.07454 & .25 .75 & .14523 \\
\hline .00259 & - 60406 & 2.19040 & .165113 & -.16173 \\
\hline - buars & C & .45120 & -.25471 & -.18986 \\
\hline - $\operatorname{lon} a$ & - Drfign & 1.10074 & .15080 & .07890 \\
\hline - Crowas & - 39cuc & .18790 & -.24449 & -.07454 \\
\hline - Conto & - & $-2 \cos$ & .6507 & 2.19840 \\
\hline 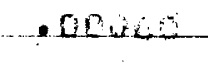 & a 1014 & - & -0040 &.$\$ 5120$ \\
\hline - Obsur & - vinesa & 04003 & - Udstse & 1.00790 \\
\hline - OODLO & - $y=96$ & - $C 0(150)$ & .4000 & .18790 \\
\hline .00000 & - Ugrax & - Cbibigi & - 14000 & - wanon \\
\hline- Cobiso & $\rightarrow 00100$ & - Leilus & $-\operatorname{Ler} 2 u$ & .40504 \\
\hline - frotage & - byongen & - Govara & .00250 & .00000 \\
\hline - (rublis & - Utsucit & - Uchiss & - 40000 & - 00000 \\
\hline - cilcitas & - yotikn & $-60 t+0$ & .0009 & $.00 \mathrm{cos}$ \\
\hline a Cincis & - Goul & Q UECLO & - Duren & - anuto \\
\hline - Mrander & - Gmarar & - bracisar & - Uriobsh & - Ucilou \\
\hline - oubua & $-000 L O$ & .0000 & - 00000 & .00000 \\
\hline 11. & 12. & 13. & 14 & 15 \\
\hline
\end{tabular}




\begin{tabular}{|c|c|c|c|c|}
\hline .07653 & -.12771 & - 43862 & -.05878 & .03244 \\
\hline-01176 & -0.0727 & -162.0 & -.08073 & -.01963 \\
\hline .05219 & $-.1598 k 1$ & .02415 & -.112918 & -.184694 \\
\hline .00491 & -0019781 & $\cdot \operatorname{sig} 274$ & .00586 & .06074 \\
\hline .07411 & -.22537 & .27053 & -.12771 & .03862 \\
\hline-00315 & -10349 & -21176 & -.99329 & -.01620 \\
\hline .054838 & $-.17: 47$ & .15219 & .09811 & - 42415 \\
\hline - 0,519 & - is 1585 & .20491 & -.010781 & .000274 \\
\hline .05773 & -.11536 & .47411 & -.22537 & .07053 \\
\hline-000547 & $-.11: 77$ & $-\operatorname{sic} \theta 15$ & -10349 & $=41176$ \\
\hline .04636 & -.15834 & .45488 & -.17147 & .015219 \\
\hline .05352 & -00872 & .50519 & -.01585 & .02491 \\
\hline .04575 & -.21335 & .05773 & -.18536 & .197411 \\
\hline$=00456$ & -11889 & $=\sin 597$ & -.11092 & -.40815 \\
\hline $.03: 03$ & $-155 ; 22$ & .4636 & -.15834 & .135488 \\
\hline .036486 & -011238 & .60352 & -00872 & .06519 \\
\hline-.112516 & -.0671 & .04575 & -.21735 & .45773 \\
\hline$-0412=2$ & -11565 & $=20456$ & -11889 & $=01592$ \\
\hline-.01914 & -.11745 & $\therefore 03063$ & -.15522 & .04636 \\
\hline-.00117 & .03644 & .30486 & -.01238 & .00352 \\
\hline-.01080 & -11328 & -.02516 & -.06710 & .0 4575 \\
\hline-01865 & $-.115,5$ & $=-r_{4} 732$ & -11565 & $=24456$ \\
\hline-.01586 & -13448 & $=01914$ & - . 3740,5 & .03063 \\
\hline .00198 & -.015212 & -00117 & .10644 & .90486 \\
\hline-.03497 & .17719 & -0.1680 & -.11328 & -.12516 \\
\hline-0.0728 & -.12293 & $=01065$ & -.11525 & -141702 \\
\hline-.14149 & .1456 & -.41586 & -.133648 & -.01914 \\
\hline .25 .95 & .94523 & - LEL 198 & -.09212 & -.04117 \\
\hline .16515 & -16173 & -.3497 & .17719 & -.01080 \\
\hline$=25472$ & -18986 & $=.4228$ & -.49293 & -.013665 \\
\hline .15182 & .67098 & -.1440 & .10456 & -.1586 \\
\hline$-.2444 \pi$ & -0.07454 & .25095 & .04523 & .12198 \\
\hline - laficares & $2.1984 n$ & .16510 & -.16173 & -.113497 \\
\hline - Condes & .25120 & -.25470 & -.03986 & -.05728 \\
\hline - conatios & 1.00796 & .15080 & .07890 & -.01440 \\
\hline - Govars & .18790 & -.24448 & -.07454 & .25995 \\
\hline $.00 \mathrm{cos}$ & - Liflas a & .00004 & 2.19846 & .16510 \\
\hline OIOLLU & - L LIIILil & $\rightarrow$ ULLibi & .55126 & -.25470 \\
\hline - nombi & - enisst: & - 6oboge & 1.011798 & .15080 \\
\hline - ogoger & - vued: & - dousu & .18790 & $-.2444 u$ \\
\hline 16. & 17. & 18. & 19. & 20. \\
\hline
\end{tabular}




\begin{tabular}{|c|c|c|c|c|}
\hline .02864 & -.0272 & - 7965 & -.04863 & .07353 \\
\hline-.017373 & -.52133 & -.127171 & -.42130 & -.07411 \\
\hline .04012 & -.03136 & .88360 & -.114786 & $.1,7496$ \\
\hline$\cdot \arg 4 \cdot 14$ & $-0 \sin 11.3$ & -10314 & -.60263 & .00281 \\
\hline-.115878 & .61244 & $\cdot 22864$ & $-.1 \geq 2782$ & .07965 \\
\hline-18.873 & -131763 & $=.7323$ & -.12133 & -.47171 \\
\hline-.02918 & $-00,694$ & $.040 \leqslant 2$ & -.03136 & .08360 \\
\hline$-.91: 586$ & .45074 & - 20014 & -.00103 & .00314 \\
\hline-.12171 & - 13962 & -.5888 & .00244 & .02864 \\
\hline$-.09 \times 29$ & $-.41 \sin$ & -.28673 & -01963 & -.07373 \\
\hline-.09811 & .02415 & -.12918 & -.01694 & .04002 \\
\hline-.00781 & .00274 & -0.0586 & .001974 & .00014 \\
\hline-.22537 & .47053 & -.12771 & .13862 & -.05878 \\
\hline-113349 & -.1176 & $=049429$ & $=.01620$ & -.08073 \\
\hline-.17147 & .15219 & -.9981 & .02415 & -.92918 \\
\hline-01585 & -100491 & -20781 & .85274 & -.0586 \\
\hline-.185 .36 & .17411 & -.22537 & .17053 & -.12771 \\
\hline$-11 \operatorname{lis} z$ & -.30015 & $=.16349$ & -.11176 & -.19029 \\
\hline. .15834 & .05488 & -.17147 & .115219 & $=09811$ \\
\hline-.00872 & .00519 &. .01585 & .00491 & -.00781 \\
\hline-.21035 & .05773 & -.18536 & .07411 & 0.22537 \\
\hline$=11889$ & -.045997 & $=11497$ & $-2 \sin 15$ & $=11349$ \\
\hline-.15522 & .04636 & -.15834 & .15488 & -.17147 \\
\hline-01230 & .03352 &.$- \sin \beta 72$ & - 4519 & -.1585 \\
\hline$m \cdot 10671:$ & .04575 & -.211335 & .65773 & -18536 \\
\hline-.11565 & -.1456 & -11889 & -.100597 & -11097 \\
\hline$-.074 k .5$ & -103963 & -.15522 & .194636 &. .15834 \\
\hline .00644 & .4498 & $=.1238$ & - 1432 & -.11872 \\
\hline-.11328 & -.02516 & $-.067 \times 0$ & .104 .575 & -.21035 \\
\hline$=11505$ & -24072 & -.11505 & -.00456 & $=11889^{\circ}$ \\
\hline-.03348 & .01914 & $-.674,5$ & .0363 & -.15522 \\
\hline-.00212 & -.9117 & $\therefore .044$ & - 04t 86 & $\because .1238$ \\
\hline .17719 & -.01080 & -.11328 & -.12516 & -.016710 \\
\hline-.09293 & -0.06665 & $=i 15 \times 5$ & $-.04 Z: 2$ & -11565 \\
\hline .11756 & -.01566 & -0.36148 & -.11914 & -.117405 \\
\hline $.04 \vdots 23$ & .04198 & $\Rightarrow 212$ & -.00117 & - 03044 \\
\hline-.16173 & -.03497 & .17719 & -.011080 & -.11328 \\
\hline-1083986 & -29728 & -19243 & -.04665 & -11505 \\
\hline .07897 & -.01443 & .10456 & -.01506 & .0 .03048 \\
\hline-.07454 & $\cdot 25: 95$ & .04523 & .00198 & -.11212 \\
\hline 21. & 22. & 23. & 24. & 25. \\
\hline
\end{tabular}




\begin{tabular}{|c|c|c|c|c|}
\hline-.04215 & .01453 & -.11789 & -.07130 & .01606 \\
\hline-.1733 & $-0.5755 !$ & -.41678 & $=.08173$ & -.01477 \\
\hline-.0 .4129 & 0.12 .267 & -.11889 & -.05177 & .01068 \\
\hline-0.11214 & -00558 & $-\operatorname{1n} 1006$ & -00552 & .00110 \\
\hline-.14863 & .07353 & -.04215 & .01453 & -.1789 \\
\hline-12133 & -.07411 & $\therefore \ln 143$ & $-.0785 \mathrm{E}$ & -01678 \\
\hline-.04736 & .07446 & 0.6 .4129 & .112267 & -.01889 \\
\hline$-.002 b 3$ & -0112281 &.$- \ln 2.14$ & -.010058 & -00086 \\
\hline$-.0 .27,1.2$ & .07765 & $\therefore 4403$ & .07353 & -.04215 \\
\hline-013133 & $=.42171$ & $=12130$ & -.117411 & -201433 \\
\hline-03136 & $.03936 \%$ &.- .14786 & .117496 & -.14129 \\
\hline-.01103 & - 100314 & $-\sin 263$ & .19281 & -.010214 \\
\hline .012244 & .02864 & -112762 & .137965 & -.04863 \\
\hline-.11963 & -.47373 & -.42133 & -0.7171 & -.12130 \\
\hline-.196694 & - 1402 & -13136 & .08360 & $=.34786$ \\
\hline .01474 & -20014 & -06103 & .810314 & -.100263 \\
\hline .03862 & -.15878 & 10244 & .02864 & -112702 \\
\hline$-0162 r$ & -243473 & $=.111463$ & -0.07373 & $-.12+33$ \\
\hline .02415 & -.42918 & - 1:E694. & $.1: 4: 39: 2$ & $=.133136$ \\
\hline .00274 & -.43586 & .40574 & .00014 & -.00103 \\
\hline .117553 & -.12771 & .133862 & -.05878 & .00244 \\
\hline-0.12176 & -.139029 & -.11020 & -08173 & -.41963 \\
\hline .135219 & -0.17811 & 112415 & -.12918 & $=.110094$ \\
\hline .00481 & -.01078 & .103274 & -.02586 & .00074 \\
\hline .07411 & -.22537 & .370 .53 & -.12771 & .3362 \\
\hline-003015 & $=13342$ & -1176 & $=09029$ & -11620 \\
\hline .05488 & -.17147 & .15219 & -.09811 & .12415 \\
\hline .60519 & -01585 & .00491 & -.00781 & .109274 \\
\hline .115773 & -18536 & .07411 & -.22537 & .07053 \\
\hline$=00597$ & $=211092$ & $=0815$ & -.0349 & -11176 \\
\hline .04636 & $-.15+34$ & 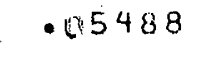 & -.17147 & .05219 \\
\hline .00352 & -010872 & - 4519 & -.1585 & .03491 \\
\hline .174575 & -.211935 & .55773 & -.113536 & .0 .7411 \\
\hline-.019456 & -11009 & -.4527 & -.11297 & $=00015$ \\
\hline .03163 & -15522 & .04636 & -.15834 & $\cdot 45488$ \\
\hline .00486 & -.01238 & .44352 & -.04872 & .04519 \\
\hline$-.025: 6$ & -.116719 & .04575 & -.2135 & .45773 \\
\hline-.00722 & -.11565 & $=0456$ & $=11889$ & $=04597$ \\
\hline-.01914 & -.074 .5 & .4363 & -.15522 & 0.04636 \\
\hline-.00117 & .10364 & .00486 & -.01238 & .00352 \\
\hline 26. & 27. & 28. & 29 & 30. \\
\hline
\end{tabular}




\begin{tabular}{|c|c|c|c|c|}
\hline-.16529 & - 050963 & -.22134 & .06883 & -.22423 \\
\hline-008161 & $=.41116$ & -17616 & -.01561 & -06610 \\
\hline$-13 \mathrm{kin} 1$ & .03994 & -.17541 & .015478 & -.17376 \\
\hline-.111144 & .4325 & -.61478 & .00432 & -.1522 \\
\hline$-.0713 \pi$ & .01616 &. .16529 & .050063 & -.22134 \\
\hline-.98173 & -11477 & -.28161 & $=01416$ & $=.07616$ \\
\hline-.05177 & $.31196 B$ & -.13001 & $.1399 \omega$ & -.17541 \\
\hline-.0552 & $.0 \vdots 114$ & -.31144 & .06325 & -.91478 \\
\hline .01453 & -0417139 & -.7130 & .0116126 & -.16529 \\
\hline-.07852 & -.01670 & -.18173 & -.01477 & -.08161 \\
\hline .02267 & -.01889 & -.15177 & .01068 & -.13001 \\
\hline$-\cos 58$ & -.914186 & $-\because 6552$ & .00118 & -001144 \\
\hline .07353 & -.154215 & .31453 & -.01789 & -.07130 \\
\hline$=0.0411$ & $=-11933$ & -23850 & -.11678 & -.18173 \\
\hline .137446 & $-\cdot 14: 29$ & .02267 & -.01889 & -.05177 \\
\hline .00281 & -00214 & $-\cdot \cos 58$ & -.00086 & -.00552 \\
\hline .117465 & $=04863$ & .7353 & -.04215 & .1453 \\
\hline$\because 47 i 21$ & $=02130$ & -0.2411 & $=01933$ & -.07850 \\
\hline .018360 & -.114786 & .07496 & -.04129 & .12267 \\
\hline-00314 & -.40263 & .00281 & -.00214 &. .00058 \\
\hline .12864 & -.0272 & .07965 & -0.04863 & .157353 \\
\hline-.47323 &.- .22133 & -27121 & $=1213:$ & $=.57411$ \\
\hline $.04 k 2$ & -03136 & .08360 & -004786 & .07496 \\
\hline$-\log 14$ & $=\operatorname{ved} 13$ &. $\operatorname{lin} 314$ & -.04263 & - 40281 \\
\hline-.175878 &. $\sin 244$ & .22864 & $-.0,2702$ & .117965 \\
\hline-.08473 & -01963 & $=7233$ & -.12133 & -.52171 \\
\hline-1.2918 & -.113694 & .64032 & -.83136 & .48363 \\
\hline-119536 & - 64074 & .20114 & -.00103 & .24314 \\
\hline$\cdots 12771$ & $.4 i 3862$ & -0.51378 & .01244 & .02364 \\
\hline-09629 & $-1162=$ & $=.2 x+3$ & -.111963 & -.67373 \\
\hline-.09811 & .12415 & -.12918 & -.00694 & .64002 \\
\hline-.130781 & .1274 &.$- \operatorname{lig} 586$ & - 34574 & $.30 \cup 14$ \\
\hline-.22537 & .07553 & -.12771 & .03862 & -.15878 \\
\hline$=.119349$ & $-.011: 26$ & $=1922$ & -.11620 & -.8073 \\
\hline-.17147 & .05219 & $=19811$ & .02415 & -0.02918 \\
\hline - 11585 & .31491 & - . 107 is 1 & .50274 & -.40586 \\
\hline-.18536 & .07411 & -.22537 & .07053 & -.12771 \\
\hline$=.11297$ & -.11015 & -.11349 & -.31176 & $=.99029$ \\
\hline-.15834 & .05483 & -.17147 & .0 .5219 & -009811 \\
\hline-.00872 & 145519 & -.21585 & .40491 & -.00781 \\
\hline
\end{tabular}

32

33.

34.

35. 


\begin{tabular}{|c|c|c|c|c|}
\hline .06479 & -.17253 & .044121 & -.07937 & .00127 \\
\hline$-.018 \% 3$ & -05354 & -12.257 & $=04145$ & -.02597 \\
\hline-0.50221 & -12591 & .42837 & -.31520 & -.01492 \\
\hline .012413 & -01230 & $\cdot .6273$ & -.600677 & -160036 \\
\hline .06883 & -.22423 &. .56479 & -.17203 & .64021 \\
\hline .01561 & $=.46613$ & -.11873 & -05354 & $=.02257$ \\
\hline .05478 & -.17 .376 & -135621 & -.12591 & .132837 \\
\hline .00432 & -01522 & $\cdot 34413$ & -0.11235 & .40273 \\
\hline $.05 i+63$ & -.22134 &. .168383 & -.22423 & .06479 \\
\hline-0.1416 & -.07616 & $=1501$ & -00610 & -.41873 \\
\hline $.0394 \pi$ &. .17541 & .05478 & -.17376 & .45021 \\
\hline - 010325 & -.41478 & .09432 & -.1522 & .10413 \\
\hline .01606 & -.16529 & $\therefore 5863$ &. .22134 & .06883 \\
\hline$=01477$ & $-4 B L 61$ & $=.1416$ & $=.07616$ & -.1561 \\
\hline $.01: 633$ & -.13341 & -13946 & .17 .541 & .05478 \\
\hline .90110 & -.41244 & - 425 & -.01478 & .03432 \\
\hline-.191789 & -.07130 & $\because 1606$ & -.16529 & .15063 \\
\hline-11678 & $-\operatorname{lis} 8173$ & -01477 & $=08+61$ & -01416 \\
\hline-.01389 & -.15177 & .511368 & -.13501 & .33990 \\
\hline-.180 .86 & -.00552 & .00110 & -.01144 & .00325 \\
\hline-.34215 & .01453 & -.41789 & -.07130 & .01606 \\
\hline-14733 & $-237850^{\circ}$ & $=1678$ & $-B B \perp 73$ & $=.11477$ \\
\hline-.04129 & 19226.7 & 0.038139 & -.05177 & .151068 \\
\hline-.110214 & $=.40558$ & $=014866$ & -.00552 & .40110 \\
\hline-114863 & .117353 & $-.42 ! 5$ & .01453 & $=31789$ \\
\hline$-.021 .3=$ & -.117411 & $\because-1933$ & $-12785 \mathrm{E}$ & $=51078$ \\
\hline$=.04766$ & .07496 & -.44129 & .112267 & -11889 \\
\hline-1111203 & $\cup 4281$ & $\because-1214$ & $=.00058$ & -.40086 \\
\hline-1127.12 & .07965 & -.44863 & .07353 & -.184215 \\
\hline$=22133$ & -.512121 & $=2130$ & $=32411$ & $=611933$ \\
\hline-.013136 & .08369 & $\because .4486$ & .117496 & -.54129 \\
\hline - 190103 & - $453: 4$ & -40263 & - bu2 201 & $-012 i 4$ \\
\hline .092244 & .112864 & -.12702 & .017965 & -.04863 \\
\hline-01903 & $=.17373$ & -.2133 & -.42171 & $=.112130$ \\
\hline-.00694 & $.94 \pi 3: 2$ & -.13136 & .138360 & -.134786 \\
\hline$\cdot(19): 74$ & .00014 & - . od d I 3 & .90314 & -.00263 \\
\hline .03862 & -05878 & .012244 & - 12864 & -.02702 \\
\hline$=C 1 \& 28$ & -218273 & $=1963$ & -10373 & -02133 \\
\hline .02415 & -.02918 & - . 4094 & $.041: 2$ & -.63136 \\
\hline .00274 & -.00586 & .001574 & .00314 & -.40103 \\
\hline 36 & 37. & 38. & 39. & 40. \\
\hline
\end{tabular}


computation of $\hat{\mathrm{R}}^{-1}$ even when the choice of targets is consistent with strict policy existence. The problems of creating linear dependencies due to rounding would be enhanced by computing the dynamic multipliers and printing out the results rounded to say five decimal places and then constructing $\hat{R}$ based on those rounded figures. A more efficient technique would be to perform all computations together to ensure that the target number of decimal places are considered, depending on the capacity of the computer. In any case, the results may not be completely accurate as the determinant will be very close to zero in most cases.

Before attempting to compute the fixed target policy vector for each set of targets, the question of existence was analysed and "checked". For example, the matrix given above was inverted and then subjected to the traditional inverse test

$$
I=\hat{R}^{-1} \hat{R}=\hat{R R}^{-1}
$$

It was found that the unit matrix was obtained correct to six decimal places for FTll and in the cases where RL was included, correct to five decimal places. This indicates for FTll at least, that $\hat{R}$ has full rank although it must be conceded that rounding could have obscured any underlying linear dependencies. As a further check, the problem was then solved and the subsequent policy vector was substituted back in to generate the time paths for $\mathrm{Y}, \mathrm{FR}, \mathrm{C}$, and $\mathrm{CF}$. The computed and desired targets corresponded with a remarkable degree of accuracy.

The results of FTll were not encouraging. In fact, they could be classed as disastrous. While the solution generated the required time paths for the target variables, the time paths of the controls required to achieve the targets were completely outside the bounds of feasibility. Figure 30 illustrates the general behaviour of the controls. 


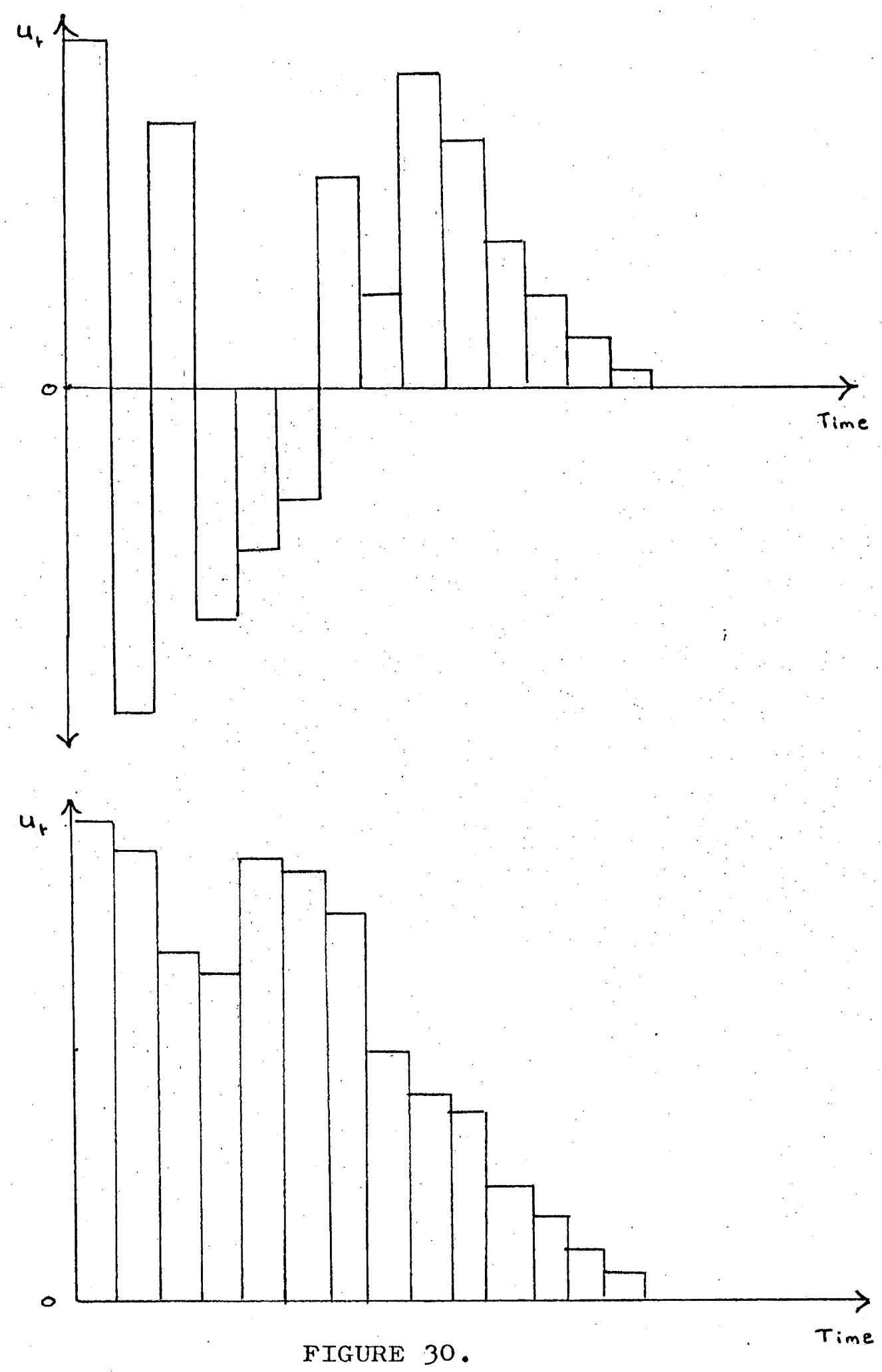

Target Path Instrument Response. 
The actual levels of the controls have not been graphed due to the wide divergence between the maximum and minimum levels of $\mathrm{G}$ and $\mathrm{DM}$ in each case. The basic tendency for the monetary controls is to fluctuate between positive and negative with the fluctuations beginning to settle down as the end of the planning period approached. The initial behaviour of the controls had the effect of fixing the initial levels of the target variables at levels far removed from their respective desired values with the target variables eventually converging on their respective desired paths. The nature of the results naturally eliminates any detailed discussion of the results as was carried out in chapters five and six but are nonetheless important in their own right. A clue to the reason behind the type of solution obtained can be found in the results of previous optimisation and strongly-Tinbergen experiments. The results of these experiments clearly show that given the model, a high degree of instrument adjustment is required to achieve exact fine tuning. The previous experiments where exact fine tuning was achieved either had the number of instruments being greater than or equal to the number of targets. Here, the number of targets is double the number of instruments and this has resulted in the amplification of the behaviour of the instruments under exact fine tuning found earlier. The results are also consistent with the internal and external balance solutions where it was found that monetary policy in particular was required to be manipulated in a severe stop-go fashion.

FTll in its original experimental framework required a target path of ten periods. Given that we could expect severe instrument adjustments, it might be reasonable to expect that the severity of the adjustment could be modified by reducing the planning period and the period over which the system is to be held exactly on target. The reduction in the length of the planning period also has the effect of 
reducing the size of the controllability matrix making the inverse and solution more accurate. A number of alternative planning period lengths were tested. Specifically, $(\mathrm{T}+\mathrm{S}+1)$ was set equal to $18,16,14,12$ and 10 periods. The policy lead and target path requirements for four targets and the above planning periods are summarised below.

Table 24

Necessary Conditions for Alternative Planning Periods

\begin{tabular}{cccc}
$\mathrm{T}+\mathrm{S}+1$ & $\mathrm{~S}$ & $\mathrm{~T}+1$ & $\begin{array}{c}\text { Dimension of } \\
\hat{\mathbf{R}}\end{array}$ \\
\hline 18 & 9 & 9 & $(36 \times 36)$ \\
16 & 8 & 8 & $(32 \times 32)$ \\
14 & 7 & 7 & $(28 \times 28)$ \\
12 & 6 & 6 & $(24 \times 24)$ \\
10 & 5 & 5 & $(20 \times 20)$ \\
\hline
\end{tabular}

The above necessary conditions of course hold for any grouping of four targets. The four target problem of FTll was solved over each of the above planning periods with the overall result that the relevant target path was achieved in each case but with levels of controls which once again displayed a considerable degree of fluctuation and lack of feasibility. Despite the failure of the solutions to provide results amenable to a meaningful economic analysis, the results do display an important general tendency. As the planning period was reduced, the degree of fluctuation in the controls became less severe. It could be argued that an improvement in the degree of accuracy of the computed inverse was responsible for such an outcome. To test this proposition the resulting inverses and inverse tests were examined very carefully. It was found that from a 14 period horizon down, the inverse became very accurate with the same general results. As a further test, the 
planning period was reduced further with inversions as accurate as that obtained in longer time paths of 14 periods or less. Once again the same general conclusion was obtained. Note that as the planning period is reduced in length we are considering smaller and smaller subsets of the controllability matrix presented above. The submatrices will converge on the top left hand corner of the matrix presented above and it can be seen that the number of zero elements will be reduced as will the number of near zero elements.

Another factor contributing to the nature of the results could be that too many targets were being aimed for in the four-target case. For experiments FTl2 to FTl4, the target set was reduced to three. The experiments are of particular importance in relation to the internal and external balance framework as the internal and external balance separation is maintained and to some extent reinforced due to the complementarity of the third target. The achievement of $\mathrm{C}$ with $\mathrm{Y}$ and FR would strengthen the internal sector as $\mathrm{C}$ is the major component of $\mathrm{Y}$ and the target of $\mathrm{Y}$ is consistent with $\mathrm{C}$ in terms of the identity, (as we have seen this does not guarantee the achievement of both in the linear/quadratic framework) and vice versa for the open sector. The close relationship between $\mathrm{Y}$ and $\mathrm{C}$ on the income side and $\mathrm{FR}$ and $\mathrm{CF}$ in the open sector suggests a priori that the adjustment in the instruments to achieve those targets would not be as severe as before. A strongly-Tinbergen analysis of the above target configurations also supports this.

The appropriate controllability matrix for the three target case can be found from the four target controllability matrix by deleting the appropriate rows. For Y, FR and C every fourth row is deleted while for $\mathrm{Y}, \mathrm{FR}$ and $\mathrm{CF}$ the third row and subsequent fourth rows are deleted and at the same time additional sets of dynamic multipliers are added for 
the appropriate targets to give the new matrix its appropriate row dimension. While the listed $\hat{R}$ will not give the full details of the appropriate three target $\hat{R}$ it nonetheless gives a clear indication as to its basic construction and properties and illustrates quite clearly that we could expect a more accurate inverse to result.

The choice of three targets with two instruments over twenty periods requires adjustment in the necessary conditions. The dynamic counting rule gives the target path a dimension of thirteen periods with a lead of seven periods. The dimension of $\hat{R}$ becomes $(39 \times 40)$. Clearly we have more time indexed instruments than is required and unless one "instrument" is deleted, $\hat{\mathrm{R}}$ will not have a regular inverse. The choice of "instrument" to drop could be quite crucial as it becomes a known exogenous variable and the value selected will influence the final result. For the initial experiments, the monetary "instrument" corresponding to the last column of the matrix listed above (column 40) was dropped and assigned a value equal to its historical level. This had the effect of allowing any level of DM to be set at the beginning of the planning period. The solution to FTl2 once again resulted in a vector of controls which could not be regarded as feasible. Similar results were obtained for FT13 and FT14. Experimentation with different deleted "instruments" did not change this basic result although the choice of instrument and the time period in which it was deleted did change the time paths of the overall control vector. The only encouraging factor to emerge was the fact that the resulting time paths for the instruments were considerably less severe than those required in the four target case. Surprisingly, the most feasible result was obtained for the target grouping, Y, C and CF. A reduction in the length of the planning period produced results similar to the four target case in that the fluctuations in the 
instruments over the entire planning period and the endogenous variables in the anticipation period were considerably less than the four target case.

Experiments FTl5 to FT19 incorporate monetary variables with income and external balance targets. For all experiments that follow, the counting rule conditions applicable to FTll to FTl4 also apply. The conclusions and results obtained from the previous fixed target experiments suggest that a similar set of results would be forthcoming for FTl5 to FT19. This indeed is the case with the added qualification that the results are more severe in relation to the required time paths for the instruments. The coupling of $M$ and $R L$ could be expected to produce some degree of fluctuation in the controls as is suggested by the strongly-Tinbergen experiments. However, the dropping of either $M$ or $\mathrm{RL}$ in a three target framework does not eliminate the severity of the control or initial endogenous variable behaviour. The results of the monetary experiments of Chapter Six suggested that if a monetary target was aimed for then the external balance position would need to be sacrificed. The strong trade-off between $M$ and FR is sufficient (in FTl6 for example) to upset the behaviour of the remainder of the system, particularly in the anticipation period. The time path for. FR fluctuates severely in the anticipation period reaching excessively high and low values thus raising the possibility of speculative capital movements. (Similar but less severe behaviour also occurred in FTll to FTl4). As with FTll to FTl4, the monetary fixed target experiments were carried out with a shorter planning period. The results reinforce those which have already been discussed. The overall results of the target path experiments indicate that the need to exactly hold targets to a desired set of paths requires excessive instrument adjustment [not unstable in the context of instrument instability]. This suggests that the achievement of a point objective may be more feasible, although it would 
be harder to justify a point objective on political and economic grounds.

The achievement of point objectives was carried out using

identical target groupings to those employed in experiments FTll to FT19 and are identified as experiments FT20 to FT28. Point controllability matrices for $\mathrm{Y}, \mathrm{FR}, \mathrm{C}, \mathrm{CF}$ and $\mathrm{Y}, \mathrm{FR}, \mathrm{M}, \mathrm{RL}$ are given below. Matrices for groupings of three targets can be obtained by deleting the appropriate row. Recall that the order of the matrix to be inverted in this case is equal to $\mathrm{m}$, the number of natural targets. Thus, for four targets a $4 \times 4$ inversion is required and it can be seen from the matrix relevant to $Y, F R, C, C F$ that it is a simple matter to establish full rank before computation. Even after fourteen periods it is a simple matter to obtain a matrix of full rank. (See appropriate matrix). The point controllability problem offers considerable scope for manipulating instruments over a variety of different time periods. For example, in the fourteen period case the necessary instrument adjustment could fluctuate between the extreme cases of taking all necessary action at the beginning of the policy lead or leaving it until the final periods. What is gained in flexibility may be lost to some degree in the need to assign values to the large number of slack time indexed instruments as the values chosen for those instruments will significantly affect the ultimate values of the instruments which are solved for. Perhaps the ideal situation would be to only consider the minimum lead necessary (see Chapter Two). In the case of four targets we would require one period lead and all the time indexed instruments would be required thus avoiding the need to assign values to slack instruments. The minimum lead approach was adopted for the two initial target point experiments in which both sets of targets were specifically aimed for. Unfortunately, the results once again must be regarded as infeasible, particularly monetary policy. The only encouraging aspect is that the required monetary and fiscal 
Point Controllability Matrix - Y, FR, C, CF •

\begin{tabular}{|c|c|c|c|c|c|c|c|c|}
\hline & \multicolumn{2}{|c|}{$\pi_{0}$} & & \multicolumn{3}{|c|}{$\pi_{1}$} & \multicolumn{2}{|c|}{$\pi_{2}$} \\
\hline $\mathrm{Y}$ & 2.1984 & .1651 & & -.1617 & -.0350 & $!$ & .1772 & -.0108 \\
\hline $\mathrm{FR}$ & .0512 & -.2547 & & -.0899 & -.0073 & ! & -.0929 & -.0067 \\
\hline C & 1.0079 & .1508 & 1 & .0789 & -.0144 & $\begin{array}{l}1 \\
1\end{array}$ & .1046 & -.0159 \\
\hline $\mathrm{CF}$ & .1879 & -.2440 & i & -.0745 & .2510 & $!$ & .0452 & .0020 \\
\hline & & 3 & & & & & & 5 \\
\hline & -.1133 & -.0252 & i & -.0671 & .0458 & 1 & -.2104 & .0577 \\
\hline & -.1151 & -.0070 & 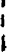 & -.1157 & -.0046 & 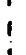 & -.1189 & -.0060 \\
\hline & -.0305 & -.0191 & $\vdots$ & -.0741 & .0306 & i & -.1552 & .0464 \\
\hline & -.0021 & -.0012 & 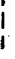 & .0064 & .0049 & ! & -.0124 & .0035 \\
\hline & & & & & & & & 8 \\
\hline & -.1854 & .0741 & ! & -.2254 & .0705 & $\begin{array}{l}1 \\
1\end{array}$ & -.1277 & .0386 \\
\hline & -.1110 & -.0082 & 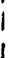 & -.1035 & -.0118 & i & -.0903 & -.0162 \\
\hline & -.1583 & .0549 & $i$ & -.1715 & .0522 & i & -.0981 & .0242 \\
\hline & -.0087 & .0052 & 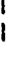 & -.0159 & .0049 & 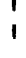 & -.0078 & .0027 \\
\hline & & & & & 10 & & & 11 \\
\hline & -.0588 & .0024 & 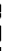 & $.0286^{\circ}$ & -.0270 & i & .0797 & -.0486 \\
\hline & -.0807 & -.0196 & 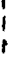 & -.07 .37 & -.0213 & $\vdots$ & -.0717 & -.0213 \\
\hline & -.0292 & -.0070 & 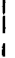 & .0400 & -.0314 & 1 & .0836 & -.047 .9 \\
\hline & -.0059 & .0007 & 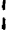 & .0001 & -.0010 & 1 & .0031 & -.0026 \\
\hline & & 12 & & & 13 & ] & & 14 \\
\hline & .0735 & -.0422 & & .0145 & -.0179 & 1 & -.0713 & .0161 \\
\hline & -.0741 & -.0193 & 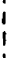 & -.0785 & -.0168 & i & -.0817 & -.0148 \\
\hline & .0750 & -.0413 & & .0227 & -.0189 & 1 & -.0518 & .0107 \\
\hline & .0028 & -.0021 & & -.0006 & -.0009 & 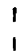 & -.0055 & .0011 \\
\hline
\end{tabular}


Point Controllability Matrix - Y, FR, M, RL.

$$
\begin{aligned}
& \begin{array}{r}
\mathrm{F} \\
\mathrm{M}
\end{array} \quad\left[\begin{array}{rrrrr:rr}
2.1984 & .1651 & -.1617 & -.0350 & .1772 & -.0108 \\
.0512 & -.2547 & -.0899 & -.0073 & -.0929 & -.0067 \\
1.4472 & 1.5283 & .4984 & -.03348 & .3782 & -.1806 \\
.0007 & -.0003 & .0008 & -.0003 & .0008 & -.0003
\end{array}\right. \\
& \pi_{3} \pi_{4} \pi_{5} \\
& \begin{array}{rr|rrrrr}
-.1133 & -.0252 & -.0671 & .0458 & -.2104 & .0577 \\
-.1151 & -.0070 & -.01157 & -.0046 & -.1189 & -.0060 \\
.0935 & -.1243 & -.7932 & .2561 & -.0815 & .3444 \\
.0007 & -.0002 & .0002 & .0001 & -.0003 & .0002
\end{array} \\
& \pi_{6} \quad \pi_{7} \\
& \begin{array}{rr|rrrrr}
-.1854 & .0741 & -.2254 & .0705 & -.1 .277 & .0386 \\
-.1110 & -.0082 & -.1035 & -.0118 & -.0903 & -.0162 \\
-1.1562 & .3386 & -1.0770 & .2972 & -.4425 & .0287 \\
-.0006 & .0003 & -.0008 & .0004 & -.0006 & .0003
\end{array} \\
& \pi_{9} \pi_{10} \quad \pi_{11} \\
& \begin{array}{rr|rrrrr}
-.0588 & .0024 & .0286 & -.0270 & .0797 & -.0486 \\
-.0807 & -.0196 & -.0737 & -.0213 & -.0717 & -.0213 \\
.1420 & -.2056 & .5934 & -.03606 & .8517 & -.4440 \\
-.0002 & .0001 & .0003 & -.0001 & .0008 & -.0003
\end{array} \\
& \pi_{12} \\
& \pi_{13}{ }_{14} \\
& \left.\begin{array}{rrrrr:rr}
.0735 & -.0422 & .0145 & -.0179 & -.0713 & .0161 \\
-.0741 & -.0193 & -.0785 & -.0168 & -.0817 & -.0148 \\
.6382 & -.3343 & .1421 & -.1199 & -.4591 & .1163 \\
.0009 & -.0003 & .0008 & -.0002 & .0003 & -.0001
\end{array}\right]
\end{aligned}
$$


policies are positive during the policy lead and target point time periods - a significant improvement over the target path results. To gain some insight into the sensitivity of the results, the policy period in both cases was extended until the fourteenth period was reached. Various combinations of slack instruments were examined firstly being assigned their target levels set out in Chapter Four, and secondly, being set at their historical levels. The overall feasibility of the results did not change but the experiments did indicate that the choice of values for the slack instruments can have a significant effect on the values of the computed control levels.

The experiments were repeated in a three target framework which eliminates the need to hit both $\mathrm{M}$ and $\mathrm{RL}$ which is a significant constraint on the solution. The dropping of $M$ or $R L$ leaving only $Y, F R, M$ or $Y$, FR, RL as the targets produced a substantially better result than the four target case but once again the policy values were not feasible and the system exhibited a tendency to fluctuate severely during the anticipation period. Note that with three targets and the minimal lead, policy planners will always require one slack instrument which makes the solution somewhat more sensitive than the four target case with minimal lead. The solutions to $Y, F R, C$ and $Y, F R, C F$ provided the best results in terms of feasibility of all the target path and target point experiments (excluding the strongly-Tinbergen approaches). The time path for $G$ with a wide variety of anticipation periods displayed a high degree of feasibility for all time periods while unfortunately the monetary instrument fluctuated between extreme and feasible positive solutions. For example, the solution to $\mathrm{Y}, \mathrm{FR}, \mathrm{C}$ with a one period lead resulted in the target point being achieved with control results for the first two periods of - 


\begin{tabular}{|c|c|}
\hline & G \\
\hline Period 1 & 747 \\
\hline Period 2 & 1168 \\
\hline
\end{tabular}

The high values of DM relative to the historical initial conditions would most likely eliminate this solution at a practical level as it would require a huge increase in domestic liquidity and corresponding shift in the private sector's portfolios. In fact the time path for DM became more excessive in terms of high and low values as the planning period progressed. That is, the target point was achieved every second period but the anticipation period was characterised by a strong movement in monetary policy, giving excessively fluctuating values for DM. This of course has a disruptive influence on the remainder of the system, particularly on FR, giving one period exactly on target and the one period significantly off taxget. The behaviour of FR suggests that exchange rate speculation could also be a problem. The results nonetheless confirm earlier experiments in that the exact achievement of internal and external balance requires a severe stop-go monetary policy - a point which is adequately emphasized by all the target path and target point experiments. The majority of the target point results confirm the target path results in that exact fine tuning results in infeasible policy requirements (remember that target path achievement implies target point achievement at the beginning of the target path) and suggest that the target path results for large controllability matrices were not a result of the possible unstable and inaccurate nature of the computed inverse. The highly accurate inverses consistent with the target point problem (see the relevant controllability matrices) which produce similar behaviour, support this contention. 


\section{LINEAR/QUADRATIC COMPARISON}

Experiments FTll to FTl9 were repeated in a linear/quadratic framework which implies infinite impatience by the policy planner in trying to achieve all objectives. The results would serve to indicate if the choice of targets would yield infeasible results in both stabilisation frameworks. The target groupings selected for detailed analysis are given in the following table. The weights for each variable are also listed.

Table 25

Targets - Linear/quadratic Comparison

$\begin{array}{lcccccccc} & \text { Y } & \text { FR } & \text { C } & \text { M } & \text { RL } & \text { CF } & \text { G } & \text { DM } \\ \text { FT20 } & 1000 & 1000 & 1000 & 0 & 0 & 1000 & 1 & 1 \\ \text { FT21 } & 1000 & 1000 & 1000 & 0 & 0 & 0 & 1 & 1 \\ \text { FT22 } & 1000 & 1000 & 0 & 1000 & 1000 & 0 & 1 & 1 \\ \text { FT23 } & 1000 & 1000 & 0 & 1000 & 1000 & 0 & 1 & 1\end{array}$

The controls were assigned a weight of one to avoid any inversion problems created by approximations, rounding and so on. All targets are in fact linearly independent and the allocation of a weight of one to the controls has the same effect as a zero weight. As before, a twenty period planning horizon was used along with multiple cost matrices to eliminate cost function bias.

The first experiment, FT20, emphasized the internal and external balance targets with $M$ and $R L$ taking on the function of intermediate variables in the sense of the definition of Chapter Four. Major results are graphed in figures 31 to 34 . The time path for $Y$ is almost perfectly stabilised in relation to its target. Any target deviations are minimal 


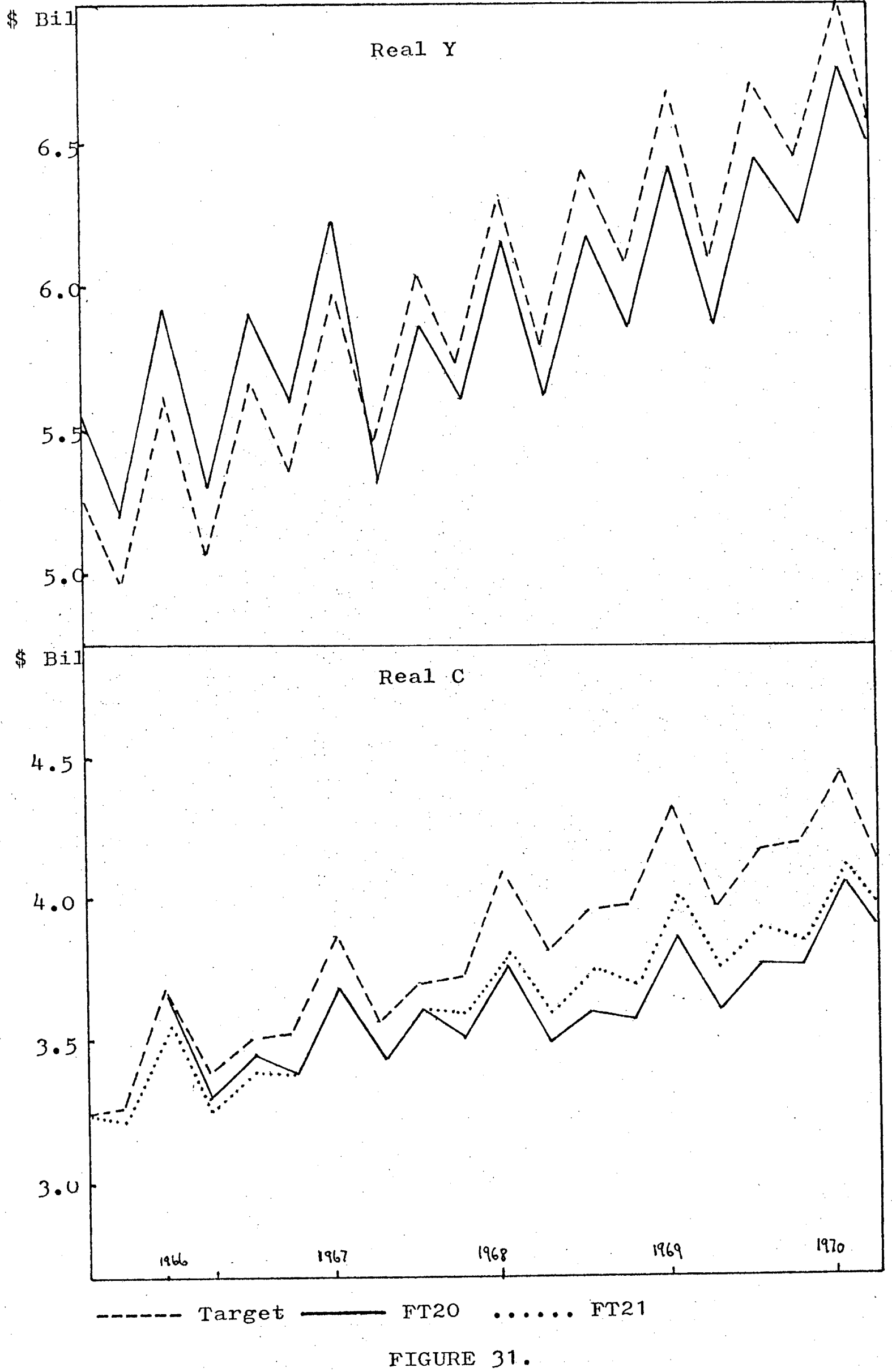




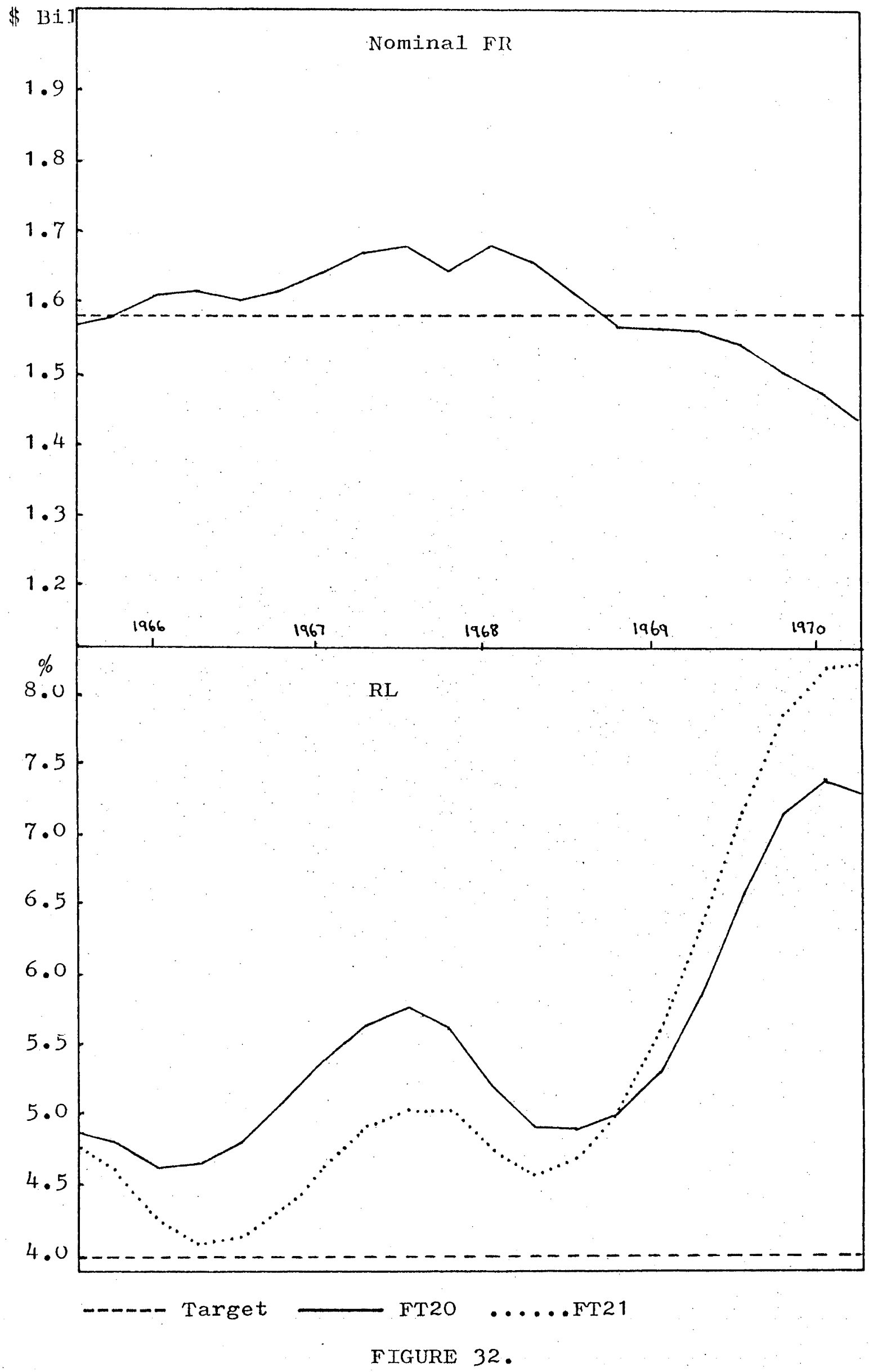




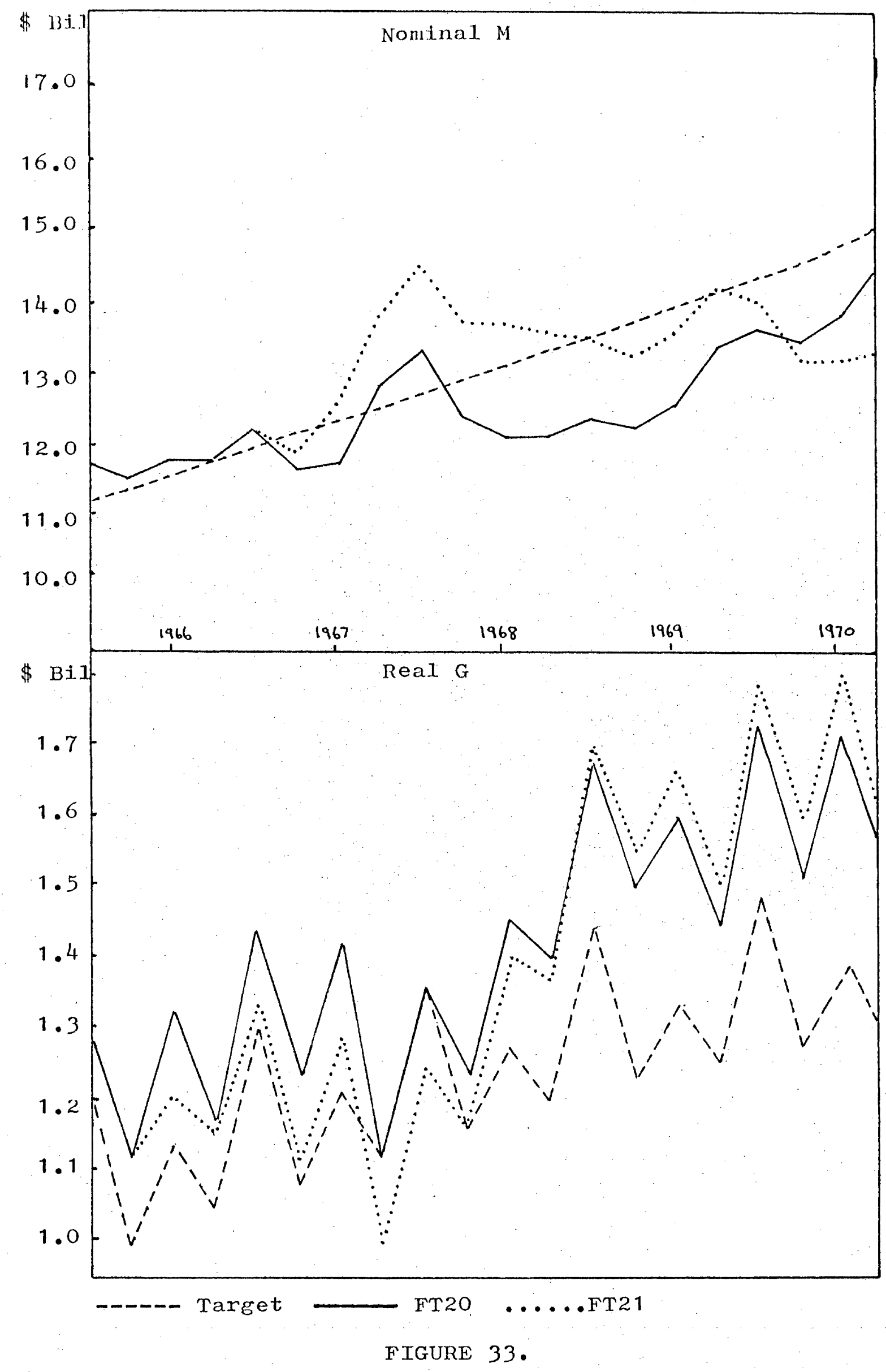




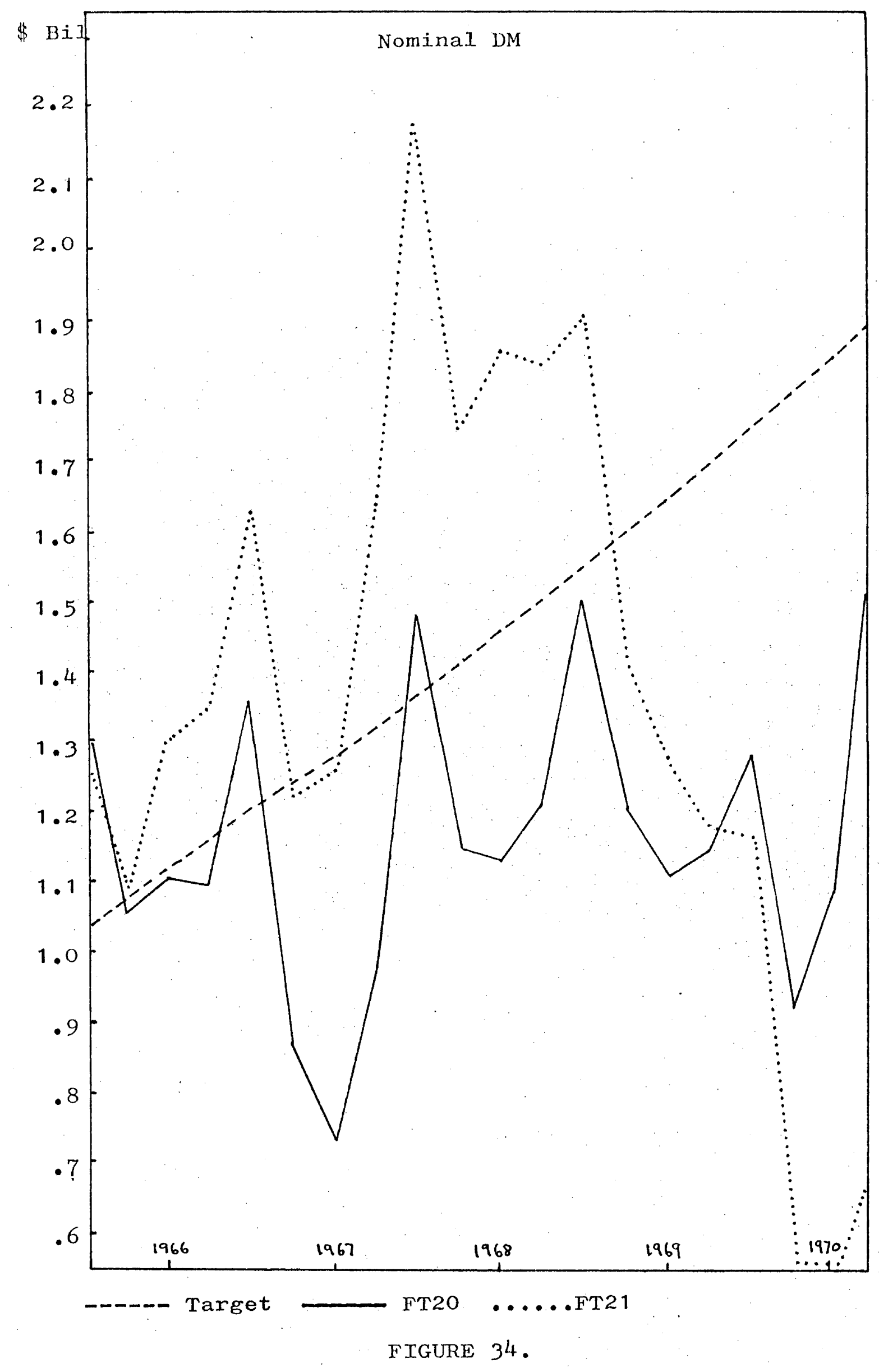


and would be of little or no concern to a policy planner in a real world situation. The optimal path for $F R$ is not as acceptable as that for $\mathrm{Y}$ although for the majority of the planning period it tracks reasonably closely to its desired path. Similarly for CF. The target which exhibits the greatest degree of fluction or divergence from its target is $\mathrm{C}$. The optimal path begins on target and then begins to systematically diverge below the desired level. It is clear that if $\mathrm{C}$ was exactly on target then the time path for $\mathrm{Y}$ would exceed its target. Recall that the target for $Y$ was specified consistently in terms of $\mathrm{C}$ and other components. Similarly, the target for FR was specified. consistently in terms of the components of the FR identity, $\mathrm{CB}$ and $\mathrm{CF}$. The results clearly show that targets which appear to be consistent at the identity level may not be consistent when a component of the identity is explained by a behavioural relationship.

The divergence of $\mathrm{C}$ from its target can be partially explained by the behaviour of the money supply which was allowed to adjust freely. It can be seen from the relevant graph that the money supply was particularly restrictive in relation to its desired time path with a severe contraction occurring in period ten. The sharp downturn in the supply of money filters through the income sector via wealth effects on consumption. Like the supply of money, the rates of interest fluctuate considerably with the long rate reflecting a "wave like" time path similar to those obtained in the internal and external balance experiments of Chapter Five with the long rate reaching a level of 7.4 at its peak. The level itself may not be too excessive but the graph of $\mathrm{RL}$ reveals that the optimal time path accelerated towards the peak in a comparatively short period which poses the problem of asset market instability and once again raises the question of whether or not some 
attempt should be made to stabilise interest rates which do respond adversely to the achievement of internal and external balance.

The results so far do not diverge that significantly from the fixed target results in that the targets are reasonably well stabilised and the intermediate variables exhibit behaviour similar to that obtained in the fixed target experiments when the target path was exactly achieved. However, the exact achievement of all targets in the fixed target framework allowed for even greater adjustment in the rates of interest and the supply of money and leaving aside questions of control feasibility, the linear/ quadratic results are preferable on these grounds. Turning now to the question of the required optimal mix of policy we can see immediately from the graphs that fiscal and monetary policy are more acceptable in this case than the fixed target framework although the results do tend to support the fixed target results. The optimal fiscal policy is exceptionally strong and expansionary in relation to its target and indicates that government spending has been almost exclusively assigned to the achievement of $\mathrm{Y}$ and C. The strong movement in $G$ over the latter half of the planning period reflects an attempt to offset the restrictive monetary policy operating on $\mathrm{C}$ and to a lesser extent on $\mathrm{Y}$. While the strong use of $\mathrm{G}$ has effectively stabilised $Y$, it is not sufficient to close the gap between optimal and desired $C$ and in fact the results suggest an exceptionally severe fiscal policy would be required to do so - a result which supports the fixed target solutions. A more severe optimal fiscal policy would have repercussions on the external and monetary sectors (see reduced form) thus requiring even greater adjustment in $\mathrm{G}$ and a significant adjustment in DM. Indeed, even without any further adjustment in G, the optimal monetary. policy is particularly severe and once again is of a stop-go nature. The general nature of the optimal monetary policy is contractionary which 
accounts for the restricted money supply and shifts rapidly between what can be loosely termed "high" and "low" values which could place extreme pressure on asset markets and in fact it may be difficult, as in. the monetary experiments, to persuade the private sector to shift its portfolios in such an extreme fashion in such a short time thus throwing doubt on the feasibility of such a policy mix.

Experiment FT21 involved the dropping of the CF target. As a consequence, the overail target performance was considerably improved. The time paths for $Y$ and FR were almost exactly on target for the entire planning period (a much superior solution to the fixed target case) and as such have not been graphed. As an indication of the increase in performance the RMSD for real Y fell from 138 for FT20 to 60 for FT2l while the RMSD for nominal FR for FT2l equalled 6 - a figure not significantly different to zero. While $\mathrm{Y}$ and FR improved considerably, only a marginal improvement occurred in C - once again illustrating the problem of consistently specifying targets. The slight improvement in $\mathrm{C}$ results directly from the more expansionary monetary policy with the significant improvement in $M$ being offset in the consumption function due to the low impact of financial wealth on $\mathrm{C}$. The other intermediate variable of importance, $\mathrm{RL}$, behaves in a more severe manner than in the previous experiment reaching a maximum value of $8.2 \%$.

The earlier IEB experiments indicated that the closer the system. moves towards exact achievement of internal and external balance, the more severe becomes the optimal policy mix. This is graphically supported and illustrated in this experiment with monetary policy becoming particularly severe moving between extremes of a significantly easy monetary policy in the middle portion of the planning period to a very restrictive policy at the end. On the other hand fiscal policy is used in a strong fashion 
as in the previous experiment but is marginally more expansionary. The dropping of one target has allowed for a greater overall controllability of the remaining targets but at the expense of a more severe mix of policy. It is interesting to compare this result with the target path and target point results. Given the same target combinations it was found that the dropping of one target (either $\mathrm{C}$ or $\mathrm{CF}$ ) had the effect of producing a less severe and more feasible policy mix - the reverse result to the linear/quadratic optimisation approach. The optimisation experiments also illustrate the advantages of reducing the gap between the number of targets and instruments. The target path approach as we have seen provides its most powerful application to situations of more targets than instruments with consequent severe repercussions for the use of the instruments. The results presented so far suggest that solely within the framework of internal and external balance, infinite impatience by the policy planner in trying to achieve his objectives and the resulting target compromise is considerably superior to anticipating a target path and achieving exact fine tuning:

The remaining two experiments to be discussed in detail feature the incorporation of monetary targets along with internal and external balance objectives. Fixstly consider FT22. The exact achievement of both $M$ and RL in a strongly-Tinbergen framework is of course not feasible due to the required policy mix and the inclusion of both targets with other targets does not enhance feasibility when anticipation is allowed. (Note that feasibility in this context does not refer to existence). The monetary target experiments of Chapter 6 illustrated the degree of trade-off beteen $M$ and $R L$ suggesting that the trading off of targets in an optimising framework would produce a situation whereby both targets would be significantly compromised. The results presented in figures 35 to 38 show the outcome to be contrary to the above and c ontrary to earlier evidence. 


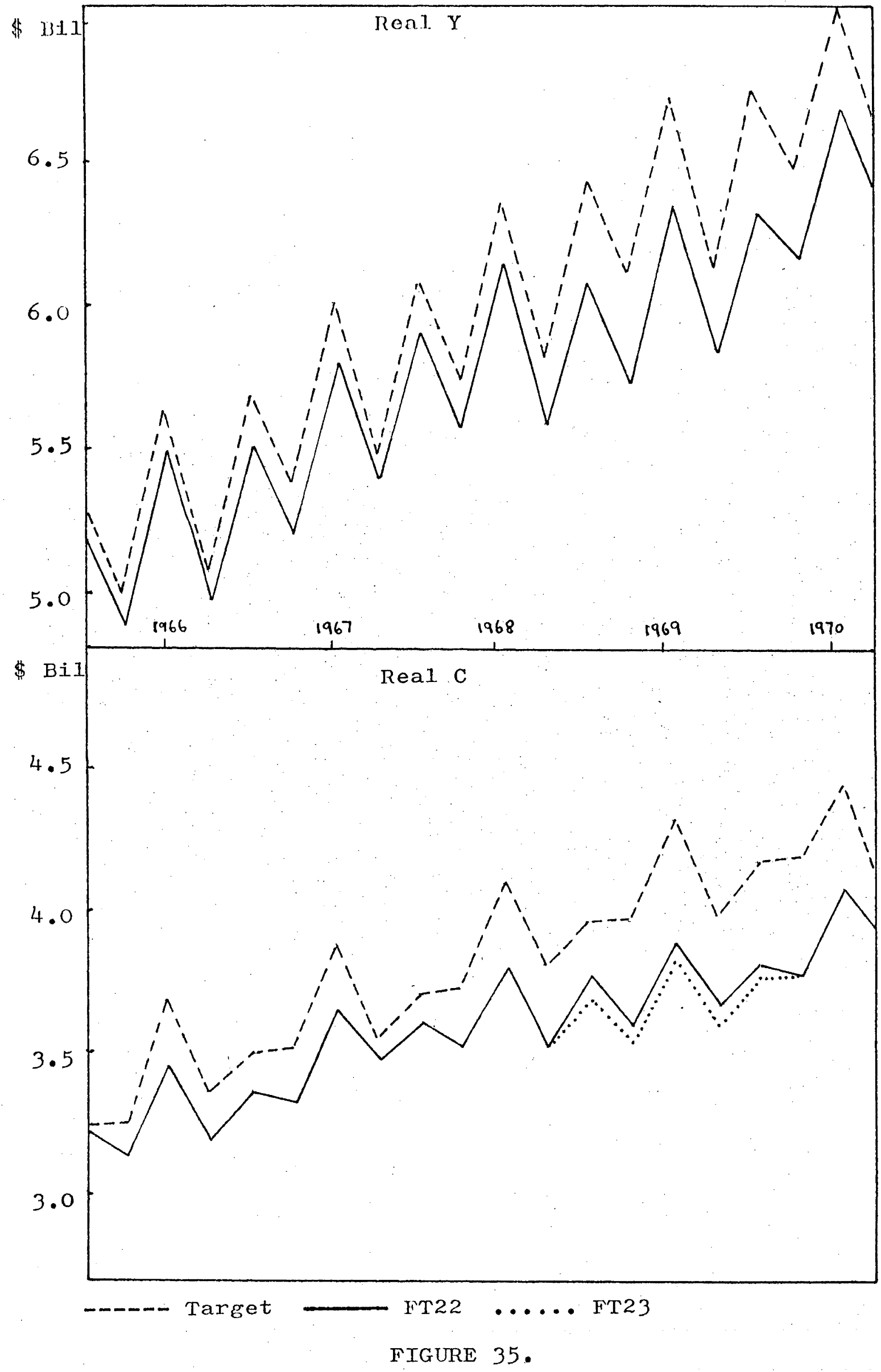




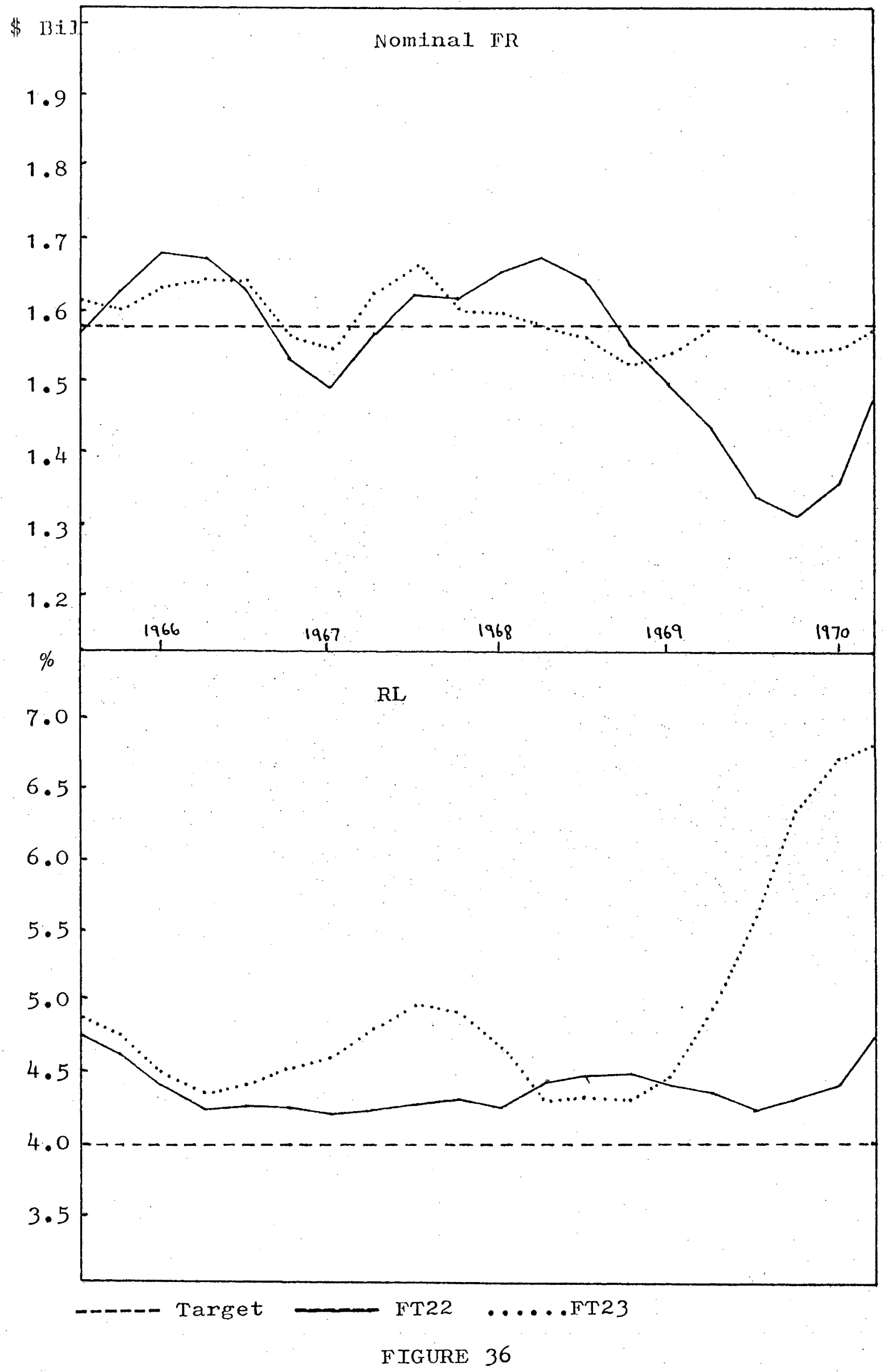




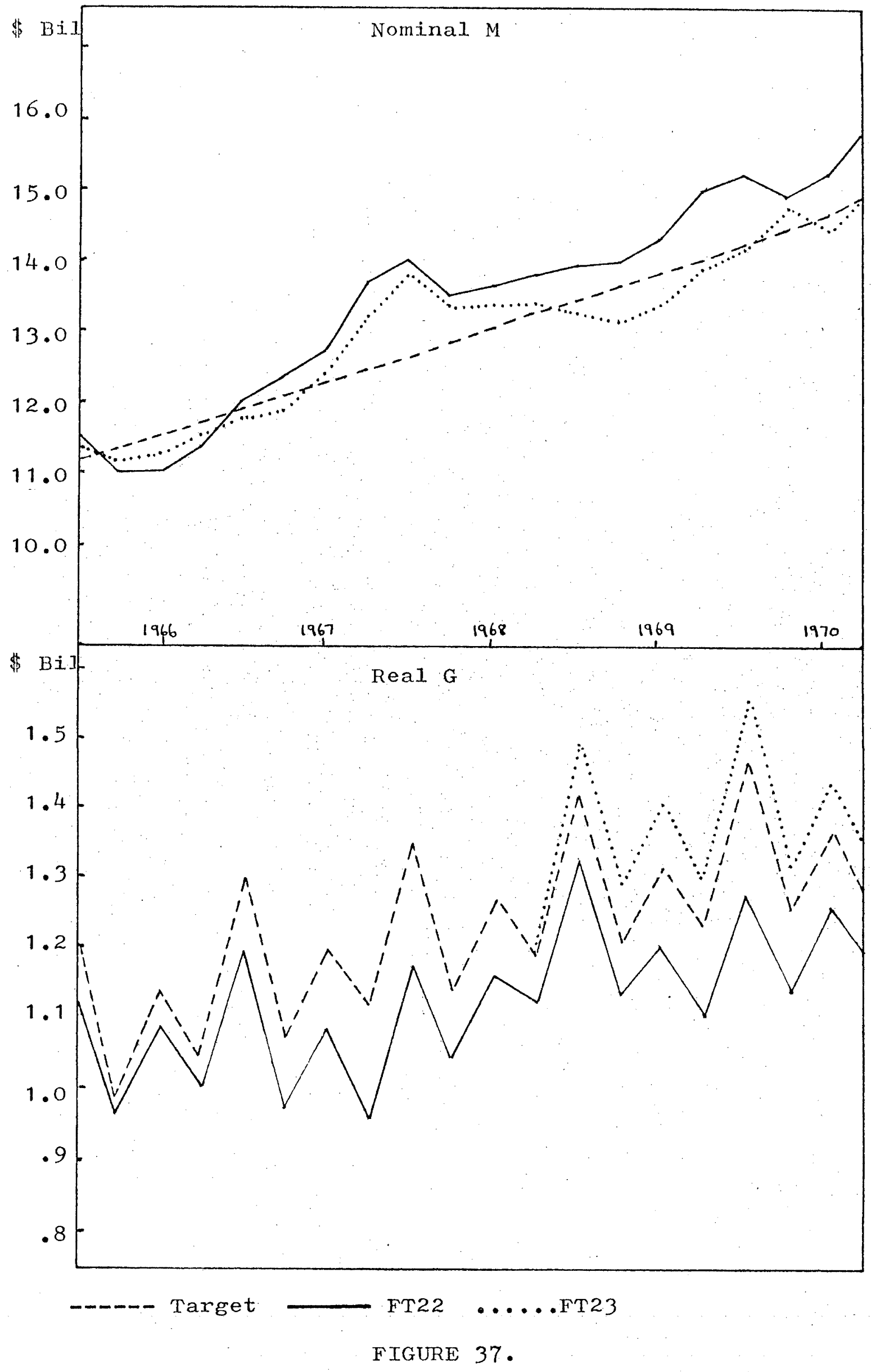




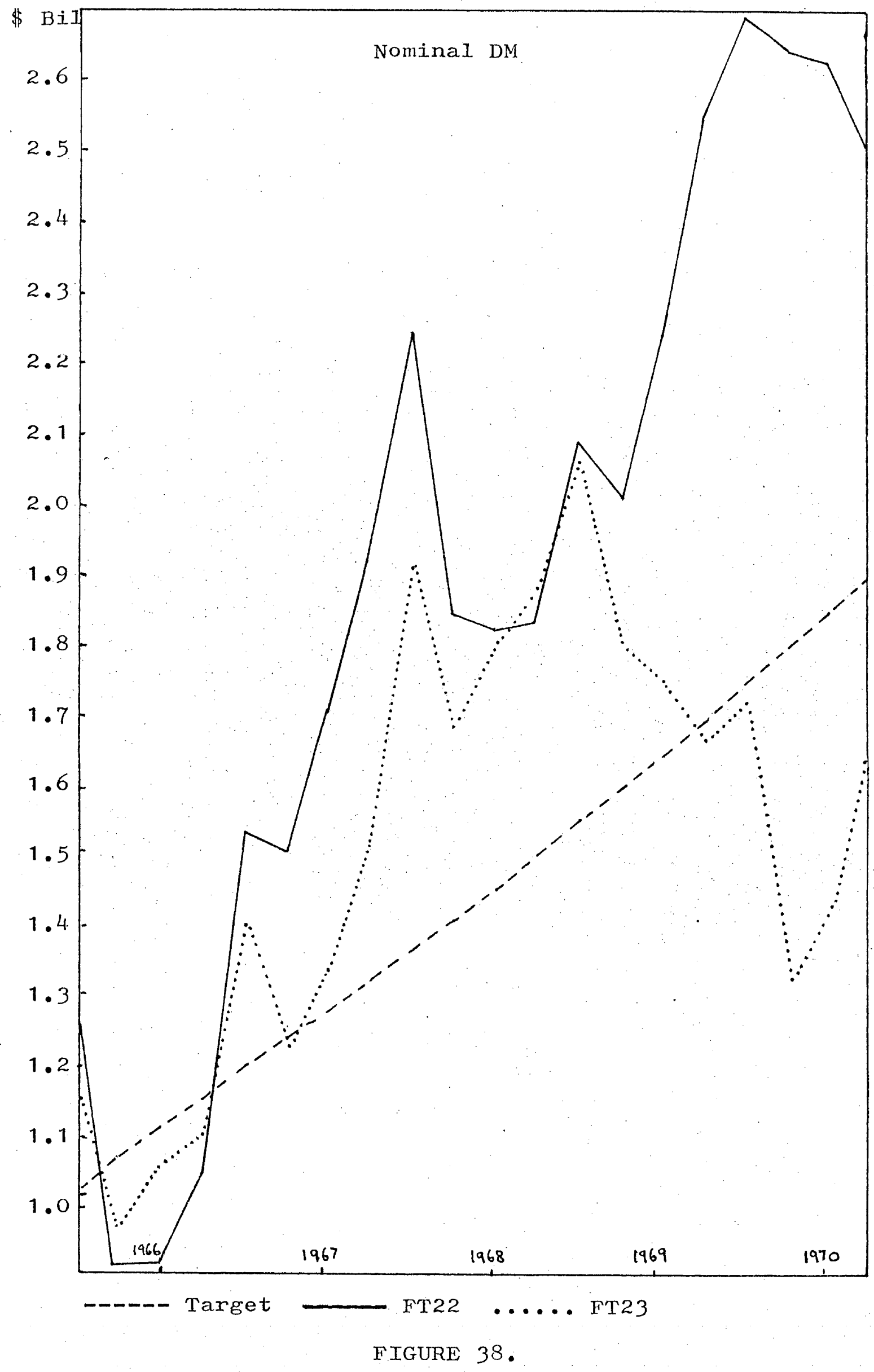


The money supply in FT22 is more effectively stabilised than in the two previous experiments where $M$ was an intermediate variable but at the same time RI has been very effectively stabilised. Indeed, allowing for some settling down of the solution in response to the divergence between the target for $\mathrm{RL}$ and the initial conditions for $\mathrm{RL}$, the optimal path presents a situation where RL has been effectively pegged. It would appear that the trade-off predicted by both the model, the earlier optimisation experiments and the fixed target experiments had been neutralised. Recall that in Chapter Six the achievement of a money supply or interest rate target produced a vigorous reaction in the balance of payments position. This result has occurred again indicating that the trade-off between $M$ and $R L$ has been partially absorbed by FR and is a direct result of the cost minimisation nature of the linear/quadratic approach. As all weights have been scaled to give identical costs for given percentage deviations, costs are minimised by restricting $M$ and $R L$ and letting $F R$ adjust in a more free manner. If the full effects of the trade-off between $M$ and $R L$ had been allowed to fully manifest itself, considerably higher costs would have been obtained. This is not to say that the trade-off has been eliminated as large percentage deviations in $\mathrm{M}$ still occur. In fact, the trade-off has been reflected more in $M$ than RL - possibly because of the presence of $R L_{t-1}$ in the function for $R \mathbf{L}$ which helps to keep $R L$ on or near target once the solution settles down to a reasonably well stabilised time path. The results illustrate how an optimising solution minimises costs by restricting the movement of variables which are likely to produce high costs, thus siphoning off any trade-off into another closely related variable - a result of particular importance when monetary targets are of concern. 
The behaviour of the income sector also deserves some comment. It can be seen from the graphs that once again income has been effectively stabilised and this result follows naturally from the monetary experiments of chapter 6 whereby it was demonstrated that a well stabilised income target was consistent with the achievement of a monetary target. The incorporation of $\mathrm{Y}$ as an explicit target along with monetary targets merely reinforces and confirms the earlier results. Once again the optimal path for $C$ exhibits large percentage deviations and as before the reason can be traced back to the optimal mix of policy. The optimal fiscal policy is significantly contractionary compared with the policies generated in FT20 and FT21 with the optimal path lying below the desired level for the entire planning period. On the other hand, monetary policy is exceptionally expansionary and severe with a massive increase in domestically created liquidity occurring towards the end of the planning period. The nature of the optimal policy is sufficient to squeeze out capital inflow in many periods. The severe expansionary monetary policy is not sufficient to produce a similar movement in the supply of money as the reaction of foreign reserves is partially offsetting and because fiscal policy is contractionary. Fiscal policy has a significant influence on the monetary sector and a restricted level of nominal income reduces the demand for money keeping interests effectively pegged at a low level. The low levels of the rates of interest contribute to the offsetting of the expansionary monetary policy. The net result is, as we have seen, a time path for $C$ which is significantly more depressed than Y. (Y lies just below its target). The deletion of the targets $\mathrm{C}$ and $\mathrm{CF}$ and the inclusion of $\mathrm{M}$ and $\mathrm{RL}$ has resulted in a switch in the emphasis on the use of the instruments from a situation of strong fiscal and monetary policy to a situation of less severe fiscal policy and a more severe monetary policy. While $\mathrm{C}$ has been deleted from the cost function, 
the overall performance of $\mathrm{C}$ is as good as in FT20 when $\mathrm{C}$ was weighted heavily. Thus, in FT2l five targets are effectively stabilised in comparison with the earlier results with the added advantage that while the relatively poor performance of $\mathrm{C}$ in FT20 and FT2l contributed substantially to total welfare costs, the same performance was maintained in FT22 with zero welfare costs (contributed by C). However FR performs less satisfactorily in FT22 than FT20 and CF in FT20 tracked very close to its target and while an added bonus in relation to $\mathrm{C}$ is gained in FT22, the overall performance is significantly worse than FT20 with total welfare costs being given by

$$
\begin{aligned}
& \text { welfare cost } \mathrm{FT} 20=3.84 \\
& \text { welfare cost } \mathrm{FT} 22=14.24
\end{aligned}
$$

which merely confirms the degree of trade-off inherent in attempting to achieve a combination of monetary and open sector targets and clearly suggests that an income and balance of payments target configuration or a configuration including other income variables and one other monetary target are preferable as regards the welfare loss generated by the quadratic cost function.

The final experiment to be discussed in detail consisted of dropping the interest rate target and attempting to simultaneously achieve $\mathrm{Y}, \mathrm{FR}$ and $\mathrm{M}$ without any policy anticipation. The major results are also graphed in figures 35 to 38 . Once again the conclusions of the monetary experiments of Chapter Six are verified (in relation to the model) in that a high degree of stabilisation has been obtained for real Y. The proximity. of the optimal path for $\mathrm{Y}$ to that of FT22 makes it difficult to graph and has not been included in figure 35. However, the dropping of RL has resulted in an improvement in the performance of $\mathrm{Y}$ illustrated by comparing the relevant RMSDs for real $\mathrm{Y}$ in both experiments. 


$\begin{array}{ll} & \text { RMSD }_{\mathrm{Y}} \\ \text { FT22 } & 197 \\ \text { FT23 } & 148\end{array}$

The improvement in $\mathrm{Y}$ has been matched by an even more significant improvement in the balance of payments position with optimal FR becoming exceptionally well stabilised, particularly towards the end of the planning period. The stabilisation of $F R$ and an improvement in the performance of the money supply has resulted in a marked deterioration in the behaviour of $\mathrm{RL}$ (see figure 36 ). This is a direct result of the trade-off between monetary variables and by letting RL take on the function of an intermediate variable, $F R$ and $M$ are able to be more effectively stabilised.

The dropping of RL from the cost function has the result of completely reversing the emphasis in the use of the instruments. Fiscal policy is more expansionary but the significant switch has occurred in the use of monetary policy with the direction of policy being substantially reversed towards the end of the planning period. Monetary policy is still severe and of a stop-go nature but the overall use of DM has been less severe than in FT22 as is indicated by the appropriate RMSDs and figure 38 . $\mathrm{RMSD}_{\mathrm{DM}}$ FT22 585

FT23 280

This result concurs with the fixed target solutions where it was found that the deletion of either $\mathrm{M}$ or $\mathrm{RL}$ as a target resulted in a relaxed use of monetary policy and once again is a direct result of the trade-off between $M$ and the rates of interest. 
STOCHASTIC EXTENSIONS

All fixed target and linear/quadratic experiments presented in this chapter were repeated within a stochastic framework. Firstly, within a monte carlo framework to obtain some sampling statistics and secondy specific illustrative sets of stocks were used and the subsequent system performance analysed in relation to instrument and target sensitivity. It was found that the same general results held for both monte carlo and illustrative single shock experiments. The failure of the target path and target point solutions to provide feasible results in a deterministic framework means that a detailed stochastic analysis of the resulting time paths is equally fruitless from an economic point of view. Suffice to say that the presence of additive uncertainty to which the instruments are unable to adjust (except in strongly-Tinbergen situations) results in the target path or target point being severely compromised and throws some doubt on the use of fixed target techniques, particularly when there is a high degree of uncertainty, on the other hand, strongly-Tinbergen solutions respond exceptionally well to additive shocks. This is of course a result of the instruments and outputs being computed for each unit time period and as the solution explicitly incorporates the past state vector at each stage of the solution, the instruments are able to adjust for past shocks. A similar result was obtained for the linear/quadratic solutions under similar circumstances and when the number of instruments exceeded the number of targets. The results of experiments from earlier chapters confirms this and as such a graphical exposition of the results has not been presented. The strongly-Tinbergen approach to stabilisation can be superior to a flexible target approach to the same problem as in some cases an optimisation approach will require a solution to settle down towards the targets. This need not always be the case as was 
illustrated by IEB2 in Chapter Five.The degree of settling down will depend on the lag structure of the system and upon the relationship between the initial conditions and the desired target paths of the outputs. A safety-first rule would be to apply a strongly-Tinbergen solution in place of an optimisation solution whenever the number of targets equals the number of instruments and of course provided policy exists. Uniqueness and existence for strongly-Tinbergen solutions and optimisation solutions are readily established from the reduced form when $m=k$ and in the case of a simple model, the conditions can be established from an inspection of the structural equations.

The stochastic results of the optimisation experiments are predictable based on the earlier work. The results clearly show that income and external targets are preferable in terms of welfare loss to income, external and monetary targets under uncertainty. The requirement that there be more targets than instruments results in the system being unable to adjust in a manner which allows the optimal stochastic paths to fluctuate about their desired levels (see IEB2 and strongly-Tinbergen results). The "best" result in terms of welfare loss from certainty to uncertainty increased in excess of 4008 . The welfare loss corresponding to FT22 and FT23 was considerably higher with the optimal path for FR being liable to decerase or increase rapidly due to the presence of $F R_{t-1}$ in the identity for FR. Similarly, the supply of money exhibited large percentage deviations. In all cases, $\mathrm{Y}$ performed exceptionally well under uncertainty, largely because the income sector is insulated to some extent from the open and monetary sectors due to the low coefficient of financial wealth in the consumption function. This result is reinforced by the fact that shocks to the income sector through C, I, TPY and so on are typically smaller than external shocks for example. The variation in 
the magnitude of the shocks reflects the real world situation where unanticipated uncertainty can arise in the open and hence monetary sector as a result of external facts being beyond the control of the domestic authorities. A comparison of the results obtained in this chapter and those of chapters 5 and 6 suggests that in the presence of an important open sector and given close linkages between this sector and the remaining sectors of the economy, the authorities should keep the relationship between instruments and targets as near as possible to equality to avoid excessive welfare loss due to uncertainty. The behaviour of the controls under uncertainty is also largely predictable based on the earlier experiments (the same set of random shocks was employed in each experiment to enable feasible comparisons to be carried out). As could be expected, the introduction of uncertainty resulted in a switch in the emphasis on particular instruments, particularly on the fiscal instrument which was employed in a relatively sedate manner under certainty but which became considerably more vigorous after the introduction of stochastic shocks. The divergence between the optimal instrument paths for deterministic and stochastic frameworks once again raises the problem of implementing a stabilisation strategy based on certainty when policy planners are forced to plan in advance of the planning period and are unable to allow the instruments to adjust in accordance with unanticipated shocks. Sufficient evidence on this is given in earlier chapters and hence will not be discussed in detail here. The RMSDs for $G$ and DM under certainty and uncertainty are given below (illustrative shocks) to illustrate the divergence in the optimal time paths and the switch in emphasis on instruments that can occur indicating the problems that could arise in implementing a successful stabilisation policy under uncertainty. 
Table 26

Instrument Performance under Certainty and Uncertainty

$\begin{array}{cccc}\text { RMSD }- \text { G } & \text { (Real) } & \text { RMSD }- \text { DM } & \text { (Nominal) } \\ \text { D } & \text { S } & \text { D } & \text { S } \\ 190 & 225 & 410 & 396 \\ 204 & 156 & 593 & 885 \\ 84 & 200 & 585 & 680 \\ 40 & 60 & 280 & 580\end{array}$

The conclusions to be drawn from this chapter are essentially clear cut. Given the model, target configurations and the numerical levels of target paths, a fixed target approach to stabilisation is inferior to a flexible target optimisation approach where targets are traded off rather than exactly achieved. The exact achievement of a target path or target point where the number of natural targets exceeds the number of natural instruments, places undesirable pressure on the available instruments. Even in strongly-Tinbergen situations infeasible solutions can arise when we attempt to achieve targets from different sectors of the system. The choice of targets employed in this study may well have been a major factor in producing extreme paths for the controls. A selection of all income targets for example may produce a set of feasible instrument time paths. Unfortunately, linear dependencies between income variables prevented a detailed examination of this aspect although the achievement of $\mathrm{Y}$ and $\mathrm{C}$ in a strongly-Tinbergen framework was consistent with a feasible set of instrument values. Additional computational experience needs to be obtained with larger models to ascertain whether or not alternative target configurations are feasible. However, the results do suggest that exact fine tuning will be inferior to attempted 
fine tuning.

The linear/quadratic flexible target solutions to the specified target configurations indicate that it is feasible to include internal and external balance targets along with monetary and other income targets. The results are very encouraging as the experiments reveal that $\mathrm{Y}$ and FR can be closely stabilised in many cases with the greatest degree of divergence occurring in other targets, for example in C. Although the trading off of targets allows for a generally acceptable overall stabilisation result, severe policy actions are required although not as severe as the fixed target solutions. It is the closing of the gap (in many cases a small gap) between optimal and desired paths in the fixed target framework which turns a severe but feasible optimal policy into a more severe and infeasible non-optimal fixed target policy. 


\section{CHAPTER VIII}

\section{S'TABILISATION AND SPECULATIVE CAPITAL FLOWS}

This chapter develops a special case of forecasting error in relation to uncontrollable exogenous variables. In particular, problems of unanticipated foreign exchange speculation are examined with specific reference to the applicability of dynamic stabilisation techniques. The results of the previous chapter suggest that a detailed analysis within the context of the fixed target framework would be largely fruitless due to the lack of feasibility of the results. As such, the emphasis will be placed on the linear/quadratic technique although it is possible to draw some very general conclusions about the fixed target approach.

The process of foreign exchange speculation essentially arises out of the utilisation of a fixed exchange rate approach to the balance of payments. If an adjustment in the exchange rate is predicted, either rightly or wrongly, then speculators will begin to move capital either into or out of the country of concern, depending on which way the exchange rate is expected to adjust. Assessments of exchange rate adjustments are usually based on the direction in which foreign exchange reserves have been moving over a particular time period. The fact that an exchange rate adjustment is expected is quite sufficient in most cases to ensure that such an adjustment does in fact occur, even if the authorities had not been contemplating such a policy decision. That is, the actions of speculators can force the expected adjustment. The problem faced by the authorities is to try to identify when speculation is occurring which may involve the difficult task of disentangling speculative capital movements from short run perturbations. A failure to correctly identify capital movements as speculative could lead to disastrous balance of payments problems with a 
corresponding effect on the money supply. Alternatively, a hasty exchange rate adjustment to eliminate what has been incorrectly identified as speculative movements could also result in unfavourable repercussions throughout the internal and external sectors of the economy.

The model as we have developed it captures the essence of this speculative interaction as can be seen from the dynamic simulation results presented in Chapter 2 (Figure 3). The massive increase in foreign reserves which occurred towards the end of the estimation period was a. direct result of speculative movements. The previous experiments conducted with the model yield some very interesting results in relation to potential speculative situations. Firstly, when FR is incorporated in the cost function (mainly with $\mathrm{Y}$ ) and controls are allowed to adjust in a free manner, $\mathrm{FR}$ is so effectively stabilised that no speculation would be likely to occur. In some instances this result holds even when the use of one instrument is restricted. Of more importance however is the result that the incorporation of $F R$ in the cost function with freely adjusting instruments is not conducive to inducing speculative movements even when additive uncertainty is introduced. The introduction of uncertainty results in a greater degree of fluctuation in foreign reserves about the desired time path but the short run shocks are not sufficient to "trigger" speculative capital flows due to the adaptive nature of the linear/ quadratic approach. The implications of this are quite straightforward and very important - if speculation is to be avoided under a fixed exchange rate regime then the authorities should exercise a considerable degree of control over the balance of payments. In the case of a country like Australia, control over the balance of trade is quite difficult due to the volatile and largely unpredictable nature of exports so attention must be focussed on capital flows. The comparative optimisation 
experiments of Chapter 7 also yield similar results. The inclusion of FR in the cost function with more than one other target also resulted in FR being stabilised at levels which would preclude any speculative reaction in relation to an anticipated revaluation. (The model assumes that speculation was triggered in 1971 at a level of $\$ 1779$ million).

The monetary experiments reveal a different reaction in the level of foreign reserves with at least one optimal path being characterised by exceptionally severe upswings and downswings (other paths exhibited behaviour of a less severe nature). Indeed, the majority of monetary experiments create conditions conducive to speculative capital movements. The situation of severe upswings (or downswings) which stabilise out at some higher or lower level presents a particular problem for the authorities. If an optimal plan is being followed then it would be necessary to publish full details of the expected movement in foreign reserves. By doing so the government may be able to prevent speculative movements from occurring by pointing out that after some initial adjustment in reserves, reserves would become stabilised. A failure to do this could of course result in the optimal strategy being destroyed although it could not be guaranteed that the publication of the desired strategy would have the desired results. The ability of the government to convince the private sector that no exchange rate adjustment will occur is extremely limited as is illustrated by recent events towards the end of 1976 when constant appeals by the government failed to prevent speculation. Although the monetary experiments of Chapter 6 are only to be viewed as polar cases, they do suggest that the pursuit of a monetary target could lead to an unstable time path for foreign reserves. It is interesting to note that since the 1976 Liberal budget there has been an attempt to steer the money supply as close as possible to a rigid target and let interest rates adjust. Towards the end of 1976 
balance of payments problems began to emerge which were primarily a result of capital flows - a result anticipated by the model although it must be stressed that the model only allows for an upward surge in reserves with. a resulting revaluation. Nonetheless, looking at the predictions of the model in reverse gives an accurate forecast.

The general conclusion to be gleaned from the preceding optimisation experiments is that so long as the balance of payments position is incorporated in the policy planner's cost function as a major target (high weight), speculative capital movements can be avoided along with a corresponding explosion in foreign reserves and resulting in a massive expansion in liquidity. This indicates that under appropriate conditions the Iinear/quadratic technique can be an effective stabilisation tool in an open economy operating under a fixed exchange rate. What of the fixed target approach? The experiments conducted with FR as a target of course resulted in the FR target being exactly achieved over the specified target path. However, the need to anticipate the target path resulted in extreme values of $\mathrm{FR}$ being required during the policy anticipation period. This would most likely lead to speculative capital movements and a subsequent revaluation or devaluation with the net result that the exact fine tuning goal of the stabilisation plan would be compromised. Not only would the foreign reserves target be compromised but so would an income target for example, due to the wealth effects in consumption with wealth being affected by the behaviour of foreign reserves influencing the supply of money. While the nature of the computed controls associated with the fixed target approach would exclude the use of this technique given the model and targets selected for this study, the results nevertheless do indicate that the use of optimal stabilisation techniques would be superior to a dynamic Tinbergen approach when a fixed exchange rate is built into the 
system. Unlike the optimisation approach, the possibility of unanticipated speculation arises in the fixed target framework even when the balance of payments position is an explicit target. The only case where a fixed target approach would be acceptable would be in a stronglyTinbergen framework with as many instruments as targets. As we have seen, this situation does not require any anticipation and foreign reserves could be exactly stabilised in each time period of the policy horizon. (See IEB2, Chaptex 5.)

The existence of a potential foreign exchange speculation situation poses considerable problems for policy planners wishing to plan policy consistently within some formal framework. The major problem being that it is very difficult to anticipate when speculative movements will be triggered and to recognise such an occurrence before it becomes a serious destabilising factor. A large upswing in reserves may occur over a short period of time which is a result of large capital movements. The capital movements may not be the result of speculation and the policy planner may involse unnecessary counteractive measures. Similarly, the reverse may occur with equally disastrous results. A failure to correctly identify the appropriate trigger level of reserves also has implications for the linear/quadratic and fixed target approaches. Both techniques require forecasts of the uncontrollable exogenous variables over the length of the planning period. Given this constraint it would seem to be desirable to be able to incorporate speculative movements within the overall plan. One method of doing this would be to select the components of the welfare function or target package and perform a computer simulation. If the simulation indicates the likely presence of spcculation then the plan can be reviscd to incorporate speculative movements. The revised plan could then be implemented. One would need 

an extremely accurate model and a profound faith in its forecasting abilities to be able to pursue such a course of action. The procedure also suffers from the requirement that the trigger level of reserves must be known beforehand and as we have argued this would be extremely difficult as the trigger level will be a function of political and other non-economic variables. Despite its limitations the above procedure still remains an option available to a planner with exceptionally good information about the evolution of the system and a high degree of control over the system.

The optimisation and fixed target experiments of previous chapters have shown that severe policy movements are required for exact fine tuning in a situation of no unanticipated speculation. The complete offsetting of speculative capital movements may require such extreme policy movements that it is not feasible both economically and politically to restore stability to the balance of payments and money supply without undertaking some degree of exchange rate adjustment. This possible outcome is supported by the historical adjustment of monetary policy whereby massive open market operations were carried out towards the end of 1972 to try and sterilise the incoming capital flows. The level of the domestic monetary base fell from \$1984 million in the first quarter of 1970 to $\$ 689$ million in the last quarter of 1972 . The actions of the reserve bank were not sufficient to perfectly sterilise the incoming capital and it is reasonable to assume that perfect sterilisation would have required a considerably more severe monetary policy than that which was carried out. This type of policy behaviour would place limitations on the incorporation of speculation in the overall plan as suggested in the previous paragraph. 
the period leading up to the 1972 revaluation also reveal another important problem. It is clear from the data that the Reserve Bank was aware of the speculation problem for some time yet no supporting action was forthcoming from the government. A not unreasonable conclusion would be that political expediency was the reason for the inaction. This is supported by the fact that a general election was scheduled for December 1972 which the Liberals subsequently lost with the necessary revaluation occurring almost immediately after the change of government. Such purely political actions are almost impossible to adequately capture in a model or comprehensive plan. Alternative scenarios could be constructed for differing political reactions but it would be difficult to ascertain which plan to implement and it would not be possible to jump from one plan to another given the need to recompute the desired plan over perhaps a shorter time period. The implementation problem discussed in Chapter 2 would also place constraints on this type of action. It should be pointed out that the incorporation of political decisions is extremely difficult in many cases and is not solely confined to the problem of adjusting exchange rates to inhibit speculation. The importance of exchange rate adjustments to primary industry in Australia makes the issue perhaps more contentious than in other countries and is one factor which is likely to severely complicate any attempt to systematically plan economic policy without resorting to a freely adjusting exchange rate.

The preceding discussion paints a fairly gloomy picture for the use of formal quantitative stabilisation, techniques in an open economy operating under a fixed exchange rate. However, the nature of the linear/quadratic solution appears to offer a potential solution to the problem. The feedback nature of the optimal control laws allows the current controls to adjust to past disturbances. Given this situation, policy 
could be expected to adjust to speculative forces which appear in $x_{t-1}$ but will not be able to adjust to current speculative movements. The exact offsetting of speculation arising in the previous time period could be compromised by the current period situation and if the unstable feedback from capital flows into foreign reserves is particularly severe, explosive behaviour in foreign reserves could still occur. The previous optimisation experiments have illustrated that the composition of the feedback matrices can change substantially between target selections and cost function weightings with the same set of targets. Thus, the degree of dependence of the controls on the lagged value of $\mathrm{FR}$ (the crucial component of $\mathrm{F}_{\mathrm{t}}$ in relation to speculation) can vary to a considerable degree with the result that in some cases the degree of feedback may not be particularly significant which would preclude any effective response in the controls to speculative capital movements. Ideally, one would like $\mathrm{FR}_{\mathrm{t}-1}, \mathrm{CF}_{\mathrm{t}-1}$ ' and $m_{t-1}$ to appear in the feedback matrices as the unstable behaviour resulting from unanticipated speculation is predominantly reflected in these variables. The model only incorporates $\mathrm{FR}_{\mathrm{t}-1}$ and the inclusion of $\mathrm{CF}_{t-1}$ and $\mathrm{M}_{t-1}$ without reason could be regarded as "fixing the results". Indeed, the practice of incorporating lagged values of all endogenous variables explained by behavioural relationships is a common feature of applied optimal stabilisation studies (Pindyck (1973a), Garbade (1975a)) and serves the purpose of spreading the response of the controls to past disturbances over the entire system (as well as ensuring a good fit to the data) and lets the controls respond directly to past shocks in the target of concern. In many cases such a model specification is not justified on any theoretical grounds and if it is not adopted then we have the situation whereby controls adjust to past shocks in variables which have little effect on the target of concern. The reader is 
referred to the discussion in Chapter 6 where monetary targets are achieved through control response to other variables. Given that policy planners would be unable to exactly predict when speculative capital movements are triggered or separate speculative movements from non-speculative movements in the initial stages of an upswing or downswing in reserves, or adequately plan for political inertia, it remains to be seen whether or not the controls can adequately adjust to speculative movements in much the same manner in which they adjust for additive disturbances. If controls are able to effectively adjust then the need to incorporate speculative movements and political decisions within the overall optimal plan will be redundant. The remainder of this chapter will focus on this issue.

\section{APPLIED RESULTS}

Before proceeding to the applied results it should be stressed once again that the results from Chapter 7 indicate that unanticipated speculative capital movements could have serious destabilising effects, even if the results obtained hud been feasible. The results suggest that the use of fixed target techniques should not be pursued when the possibility of unanticipated speculative capital flows exists. For this reason it is permissible to ignore the policy lead fixed target framework in the applied experiments that follow, with the linear/quadratic technique being of prime concern.

The overall format of the experiments is to specify a particular "trigger" level o. reserves and then re-run all previous experiments in which the trigger level was achieved. The re-run experiments allow for the speculative trigger to be activated which then sets off the unstable feedback from capital flows into foreign reserves requiring the controls 
to adjust accordingly, depending on the extent of feedback from FR $\mathrm{F}_{\mathrm{I}}$ in the feedback matrices. The resulting performance of the system can then be compared with the non-speculative results along with the ability of the controls to adequately adjust to offset the speculative capital flows. Note that the model only allows for speculative capital inflow which induces pressure for a revaluation. This is because the model was only estimated over a time period up to the 1972 revaluation and hence does not contain a proxy for speculative capital outflow and the corresponding pressure for a devaluation. Some of the earlier experiments contained results in which the time path of foreign reserves exhibited a significant downswing, the results of which cannot be directly analysed in a speculative framework due to the limitations of the model as we have seen. However, it is reasonable to assume that the unstable feedback from capital flows will approximate a mirror image effect in relation to upswing results and any broad conclusions obtained from the experiments wiil be applicable in general terms to a situation of anticipated devaluation.

The specification of an appropriate trigger level is essentially arbitrary as speculation could occur given a multitude of different levels of past reserves. Speculation could occur after a prolonged increase in reserves over time or after a short-run perturbation combined with media or other non-economic pressure which is able to persuade speculators that a revaluation is imminent. Regardless of how it occurs or at what level, it could be expected that the general reaction of the controls will be the same from case to case. To this end we will specify a trigger level of reserves which will invoke speculative capital movements regardless of whether or not reserves have been climbing steadily towards the trigger level or hit the trigger level in a short period of time. Two 
trigger levels were selected for the experiments. Firstly, a level of $\$ 1800$ million was selected and secondly, a level of $\$ 1900$ million was tested within the structure of the model. $\$ 1800$ million was selected as it is a convenient bench mark to start from given its close proximity to the historical (assumed by the model) level of $\$ 1779$ million. Only those previous experiments in which the trigger level was reached and which exhibited feasible control paths were selected. Thus, M3 (rate of interest target) was not included although the trigger level of $\$ 1900$ million was exceeded. The purpose of testing two separate triggers was to ascertain whether or not a higher initial trigger level would be consistent with greater instability than the $\$ 1800$ million case and perhaps result in an infeasible control path as opposed to a feasible control response at a lower initial level of reserves. The experiments are identified by the prefix SPEC and followed by a numeral indicating the number of the particular speculative experiment. The specific cost function weightings are set out in Table 27 wiere the letters $D$ and $S$ once again refer to deterministic and stochastic. ${ }^{33}$ where a stochastic experiment is designated it refers to the illustrative shocks discussed in Chapter 5, unless otherwise indicated. In mose cases both deterministic and stochastic solutions are relevant for a particular speculative experiment: However, in a minority of cases only the stochastic solutions are examined as the deterministic solution failed to achieve the required trigger level of foreign reserves. In all such cases the deterministic solutions failed to achieve the required level by a very small amount and in any real world situation it would be reasonable to assume that speculative capital movements would be triggered under conditions of both certainty and uncertainty. Note that Table 27 contains three different sets of experiments for each cost function specification. The three sets of experiments consist of examining the effects of 
Table 27

Speculation Experiments - Cost function Specification

\begin{tabular}{|c|c|c|c|c|c|c|c|c|}
\hline & $\mathrm{Y}$ & $\mathrm{MB}$ & M & G & DM & D & $S$ & $\begin{array}{l}\text { Trigge } \\
\text { level }\end{array}$ \\
\hline Spec $1,13,25$ & 1000 & & & .1 & .1 & & $\sqrt{ }$ & 1800 \\
\hline Spec $2,14,26$ & 1000 & & & .1 & 1000 & $\checkmark$ & $\checkmark$ & 1800 \\
\hline Spec $3,15,27$ & & & 1000 & .1 & .1 & & $\checkmark$ & 1800 \\
\hline $\operatorname{SPEC} 4,16,28$ & & & 1000 & 500 & .1 & * & * & 1800 \\
\hline SPEC $5,17,29$ & & 1000 & & .1 & .1 & $\checkmark$ & $\checkmark$ & 1800 \\
\hline $\operatorname{SPEC} 6,18,30$ & & 1000 & & 1000 & .1 & $\checkmark$ & $\checkmark$ & 1800 \\
\hline SPEC $7,19,31$ & & & 1000 & 1000 & .1 & $\sqrt{\prime}$ & $\checkmark$ & 1800 \\
\hline $\operatorname{SPEC} 8,20,32$ & 1000 & & 1000 & .1 & .1 & $\checkmark$ & $\checkmark$ & 1800 \\
\hline $\operatorname{SPEC} 9,21,33$ & 1000 & 1000 & & .1 & .1 & $\checkmark$ & $\sqrt{ }$ & 1900 \\
\hline $\operatorname{SPEC} 11,23,35$ & & & 1000 & 1000 & .1 & $\sqrt{ }$ & $\sqrt{ }$ & 1900 \\
\hline SPECl2, 24, 36 & 1000 & 1000 & & .1 & .1 & & $\checkmark$ & 1900 \\
\hline
\end{tabular}

* Experiment conducted with deterministic controls in a stochastic system..

speculation over varying time periods. By varying the time period over which speculative capital flows into the system we will be able to ascertain whether or not a cessation of such flows will enable the controls to adjust in such a manner as to allow the system to return to a position similar to the non-speculative case. That is, can the original optimal paths for the target variables be regained without the need for replanning and if re-planning is required, will it be significant in terms of the optimal plan? 
The first set of experiments (SPEC 1 to SPEC 12) consisted of allowing speculation to continue unabated to the end of the planning period, beginning in the appropriate period when the trigger level is reached (approximately period 10 in all cases). The purpose of the experiments is to detcrmine whether or not speculative flows can be sterilised in a linear/quadratic framework with the controls adjusting solely in response to the size of the feedback coefficients in the matrices $F_{t}$ relating $u_{t}^{*}$ to $F R_{t-1}$. The general feasibility results are set out in Table 28.

Table 28

General Feasibility Results - Unchecked Speculation

\begin{tabular}{|c|c|c|c|c|}
\hline Exp. & Typc & Trigger & Feasible & Infeasible \\
\hline SPEC 1 & $S$ & 1800 & $\checkmark$ & \\
\hline SPEC 2 & $D+S$ & 1800 & $\sqrt{ }$ & \\
\hline SPEC 3 & $\mathrm{~S}$ & 1800 & $?$ & $?$ \\
\hline SPEC 4 & * & 1800 & & $\checkmark$ \\
\hline SPEC 5 & $D+S$ & 1800 & & $\sqrt{ }$ \\
\hline SPEC 6 & D & 1800 & & $\sqrt{ }$ \\
\hline SPEC 7 & $D+S$ & 1800 & & $\sqrt{ }$ \\
\hline SPEC 8 & $D+S$ & 1800 & & $\sqrt{ }$ \\
\hline SPEC 9 & $D+S$ & 1800 & & $\sqrt{ }$ \\
\hline SPEC 10 & $D+S$ & 1900 & & $\sqrt{ }$ \\
\hline SPEC 11 & s & 1900 & & r \\
\hline SPEC 12 & S & 1900 & ? & ? \\
\hline
\end{tabular}

* Experiment conducted with deterministic controls in a stochastic system. 
The major result to emerge is that eight of the twelve policy selections are clearly infeasible. This is due to the fact that the controls did adjust to past disturbances but as the planning period progressed, the feedback from $\mathrm{FR}_{t-1}$ became so severe that the required monetary policy was forced to become negative. Even though extreme policy adjustment has occurred in SPEC 4 to SPEC 11 for both D and S, foreign reserves still tend to blow up towards the end of the planning period. (See figures 39 to 41 ). This illustrates quite graphically that the Reserve Bank would find it almost impossible to sterilise speculative capital movements which are allowed to continue to enter the system unabated. This is supported by the structure of the model. Of particular interest is the result that the presence of speculative capital flows overwhelms the effect of random additive disturbances in relation to the time path of foreign reserves. This is evident in the stochastic time paths presented in figures 40 and 41 . In the absence of unanticipated speculative capital flows the optimal paths for FR tend towards a significant downturn towards the end of the planning period. This is especially so in Figure 41. The shocks are unable to generate a similar downturn in the presence of speculation which indicates that while other forms of uncertainty may create a tendency to steer the balance of payments towards a substantial downturn, speculation will dominate and reverse the direction of the system.

The results of SPEC 1 and SPEC 2 are feasible in terms of the mix of policy and the presence of speculation results in an improvement in the performance of the target ( $\mathrm{Y}$ in both cases). The RMSD for $\mathrm{Y}$ in SPEC 1 with no speculation is 125 while the presence of speculation yields an RMSD of 97. The improvement is slight but it illustrates the interesting situation that speculation and additive disturbances can be 


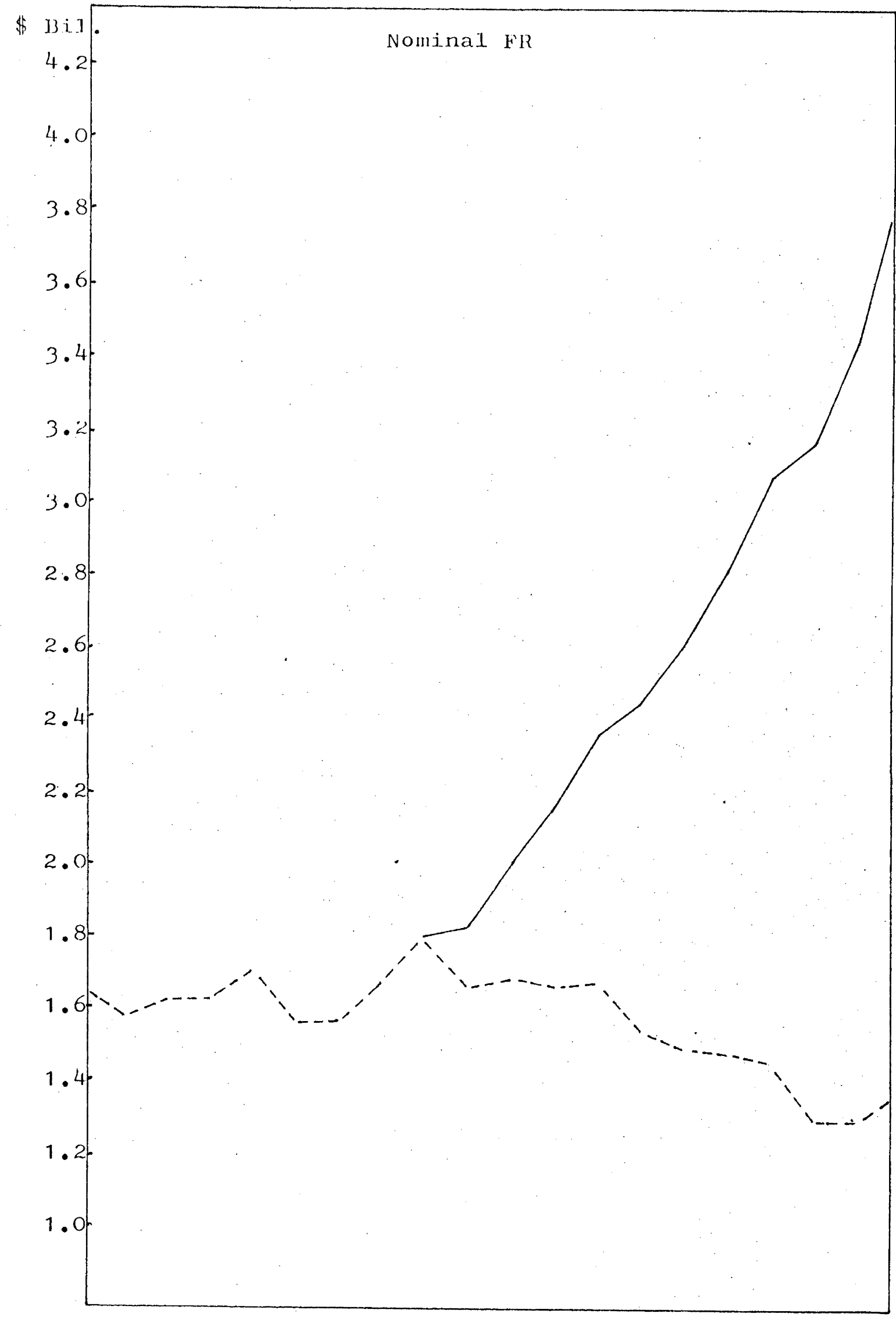

SPEC2(D) No speculation

SPEC2(D) speculation

FIGURE 39. 


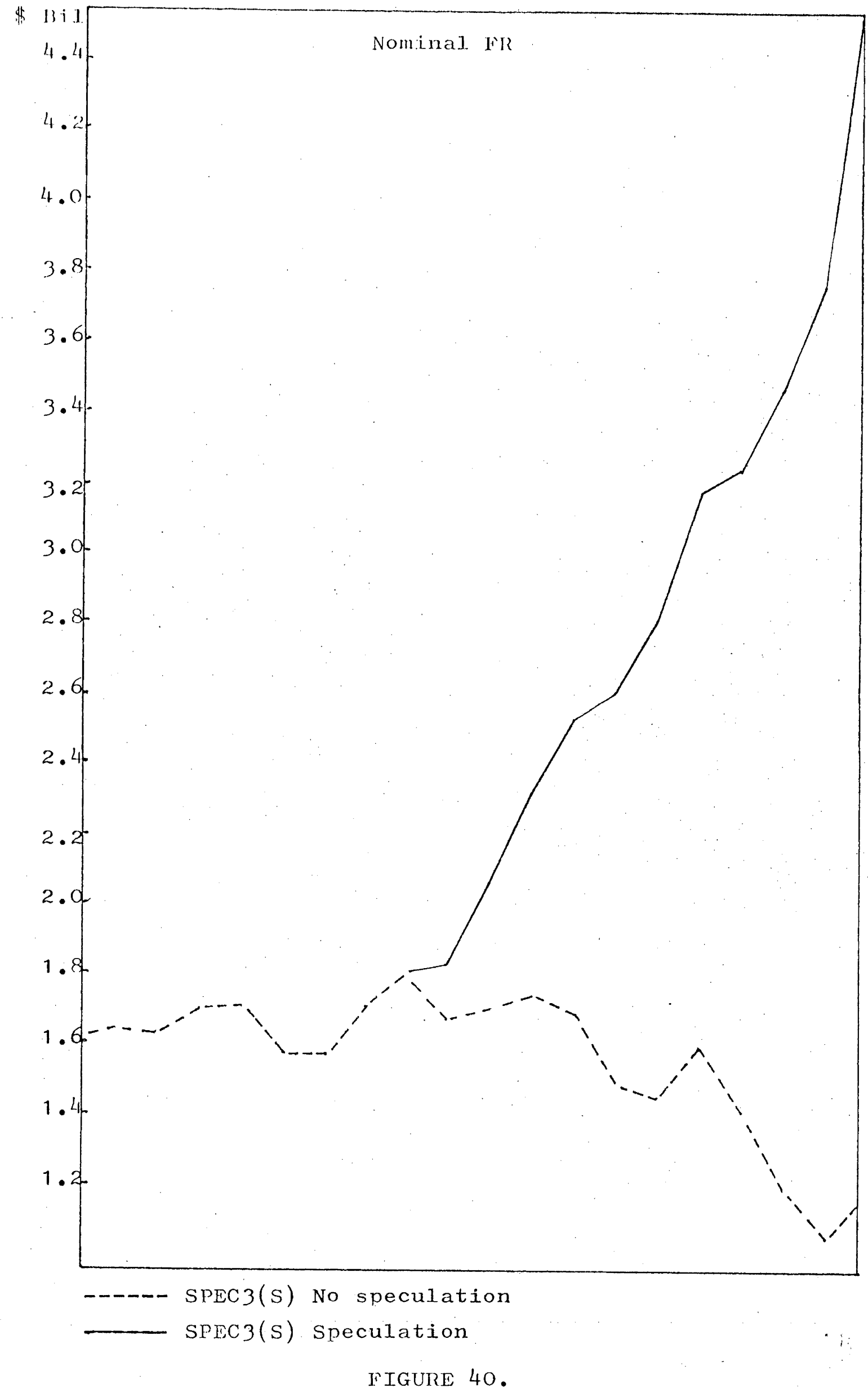




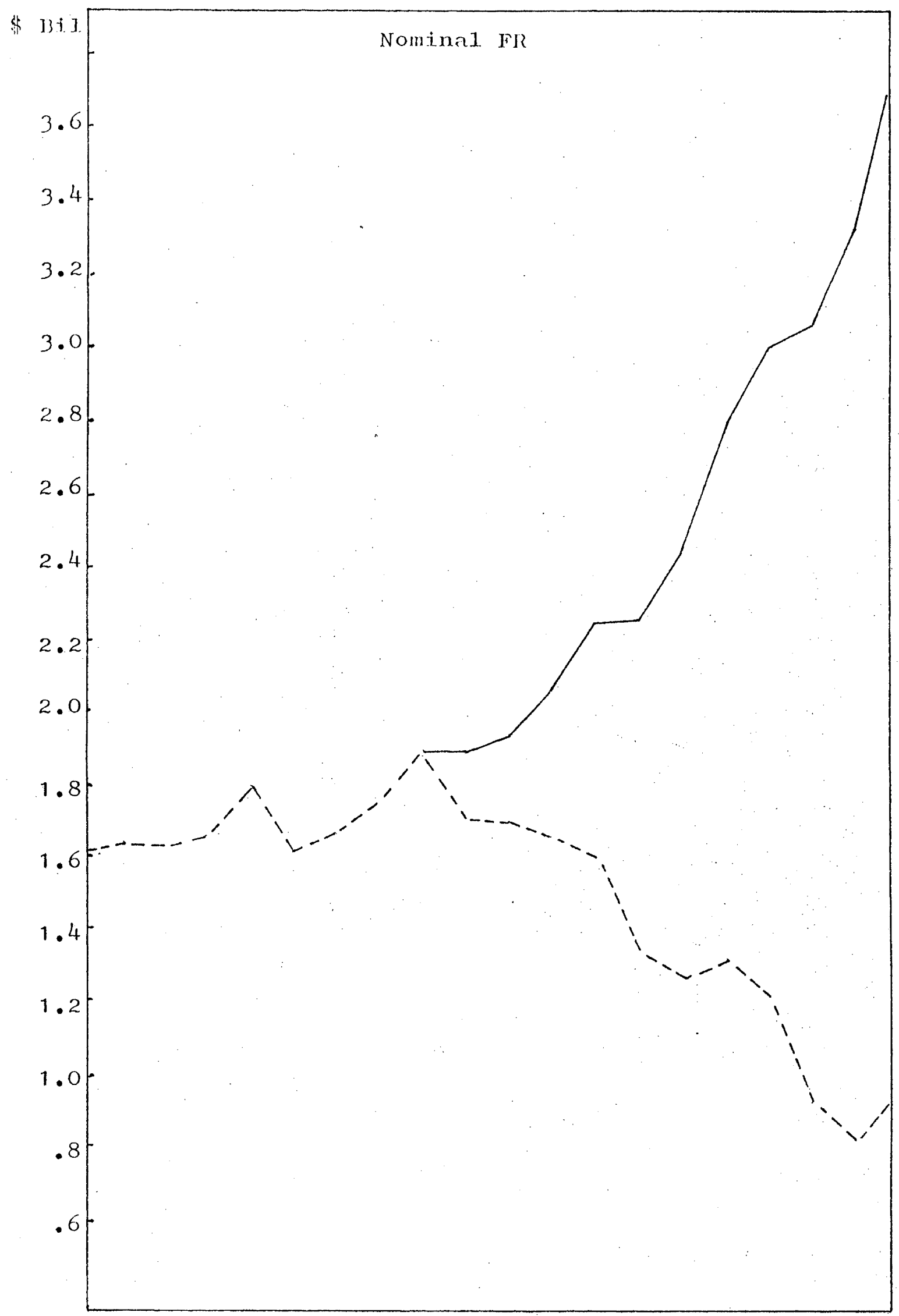

SPEC12(S) No speculation

SPEC12(S) Speculation 
complementary to the target of concern, perhaps even cancelling each other out to some extent. The time paths for speculation and no speculation do not exhibit a tendency to replicate each other but deviate from the target in different periods and with different magnitudes. Nevertheless, the general overall performance is almost identical. SPEC 2 was also feasible with the use of DM being restricted thus implying the need for $G$ to adjust to offset speculative forces on income. The RMSD for deterministic and stochastic real $G$ is given below.

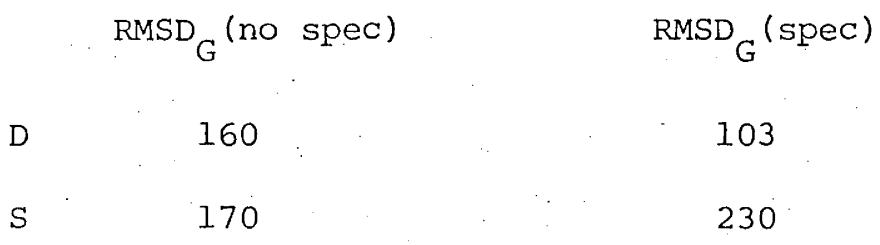

Once again the results are surprising as the deterministic solutions reveal that less fiscal policy is required in the speculative case with the result that the performance of $\mathrm{Y}$ virtually remains identical to the non-speculative case. In this case speculation has been complementary to the achievement of the target. Note that all experiments which were classified as infeasible consisted of cost function specifications which incorporated either $M$ or $M B$ as a major target and as FR has considerable influence on those variables, the feedback response was greater as was the degree of compromise with the non-speculative results. Finally, SPEC 3 and SPEC 12 have been classed as questionable outcomes. In both cases fiscal policy was allowed to adjust freely and in both cases the adjustment was relatively severe with highly depressed levels of government spending. However, the income sector was not depressed due to the upsurge in reserves and the supply of money which has a positive effect on income through the financial wealth effect on consumption. The feasibility of the results rests on a value judgement concerning the level of government spending. 
Itf a high level of social welfare and other government services are desirable then the results will not be feasible, but if the opposite is true with a high degree of emphasis on the private sector then the reverse will be true in relation to the nature of the optimal policy. A further result of some interest is that the questionable result of SPEC 12 is drerived from the same cost function specification as SPEC 9 yet SPEC 9 is clearly infeasible. SPEC 12 employs a higher trigger level which is set off in a later period than the 1800 trigger. The higher trigger level results in a slightly less cumulative feedback effect as it operates over a shorter period. This accounts for the improvement in the results. The use of a higher trigger level in other experiments did not appear to have. had any substantial effect on the general overall results.

The two final sets of experiments examine the effect of a cessation of speculative flows after a short period of operation. The implementation of this type of experiment does involve some problems however. Historically, the cessation of unanticipated speculative capital flows, whether inflow or outflow, has occurred through an adjustment in the exchange rate which naturally has had an effect on the Australian dollar value of foreign reserve holdings. The model presented in Chapter 3 does not contain any provision for exchange rate adjustments largely because the exchange rate was fixed over the estimation period and did not contribute significantly to the performance of the model. The fact that Australia did operate a fixed exchange rate explains why the estimation period ended with the 1972 revaluation. Adjustments in the exchange rate have typically been associated with a plethora of other controls designed to remove, hinder, or offset further speculative capital flows. For example, in 1972 the revaluation was accompanied by restricting the deferment of payments abroad for imports, services, dividends and interest and limiting 
pre-payments for exports. In addition, surveillance over inter-company accounts was increased and restrictions on borrowing from certain types of desposits held in Australia by overseas residents were applied along with restrictions on non-resident investment in fixed interest securities. Such controls have not been incorporated in the model and indeed their incorporation would have been difficult at the level of aggregation employed. How then do we interpret a cessation of speculation within the context of the model and the following experiments? A close inspection of the data reveals that the large 1972 revaluation had the result of firstly stopping speculation and secondly producing a revaluation of $\$ 232$ million in the Australian dollar value of reserve holdings. Given that foreign reserves stood at $\$ 5000$ million prior to the revaluation, the shift in the Australian value only constituted a small percentage change. The size of the percentage change would largely eliminate any significant adjustment in the general indicative behaviour of the economy due solely to the revaluation of foreign reserve holdings in Australian dollars. (There would be a significant change due to the cessation of speculation however). In the context of the experiments carried out in this study, the cessation of speculation after a short period with reserves at a level considerably less than $\$ 5000$ million would only require a small adjustment in the exchange rate with a corresponding slight adjustment in the Australian dollar value of foreign reserves. The net result (leaving aside a cessation in speculation) of a small exchange rate adjustment in terms of the general indicative behaviour of the model would be small and as such can be abstracted from for the purpose of a general exposition. 34 The above argument does have some empirical basis in light of the historical behaviour of the economy but if the reader is unhappy with the approach the cessation in speculation can be viewed as occurring as a 
result of strong government action in convincing speculators that the exchange rate will be defended. As before, if such an event can be carried out before speculation has been allowed to generate an apparently destabilising increase in $F R$, the approach remains quite valid.

To reiterate, the purpose of the experiments is to examine the feasibility of allowing the system to adjust to a short burst of unanticipated capital inflow due to exchange rate speculation and the ability of the system to get back on target or back to or close to the original optimal path without resorting to re-planning. The experiments consist of examining the sane trigger levels as before but incorporate two cut off points. Firstiy, the effects of a cessation of speculation after four periods is examined (SPEC 13 - SPEC 24) followed by a two period analysis (SPEC 25 - SPEC 36). As before, speculation was triggered around the middle of the planning period due largely to the impact of the exogenous variables on the system at that tine. The general feasibility results for both cut off points were identical and are presented in Table 29.

Table 29

Speculation - 4 and 2 period Cut-off

\begin{tabular}{|c|c|c|c|c|}
\hline $\begin{array}{c}\text { Exp. } \\
\text { SPEC } 13,25\end{array}$ & $\begin{array}{c}\text { Type } \\
\text { S }\end{array}$ & $\begin{array}{c}\text { Trigger } \\
1800\end{array}$ & $\underset{\checkmark}{\text { Feasible }}$ & Infeasible \\
\hline JPEC 14,26 & $\mathrm{D}+\mathrm{S}$ & 1800 & $\sqrt{ }$ & \\
\hline SPEC 15,27 & S & 1800 & $\sqrt{ }$ & \\
\hline SPEC 16,28 & * & 1800 & & $\sqrt{ }$ \\
\hline SPEC 17,29 & $\mathrm{D}+\mathrm{S}$ & 1800 & $\checkmark$ & \\
\hline SPEC 18,30 & $\mathrm{D}$ & 1800 & $\checkmark$ & \\
\hline SPEC 19,31 & $\mathrm{D}+\mathrm{S}$ & 1800 & & $\checkmark$ \\
\hline JPEC 20,32 & $\mathrm{D}+\mathrm{S}$ & 1800 & $\checkmark$ & \\
\hline SPEC 21,33 & $\mathrm{D}+\mathrm{S}$ & 1800 & $\checkmark$ & \\
\hline SPEC 22,34 & $D+S$ & 1900 & $\checkmark(S)$ & $\checkmark(D)$ \\
\hline IPEC 23,35 & $D+S$ & 1900 & $\sqrt{ }(S)$ & $\checkmark(D)$ \\
\hline SPEC 24,36 & $S$ & 1900 & $\checkmark$ & \\
\hline
\end{tabular}

* Experiment conducted with deterministic controls in a stochastic system. 
The introduction of a sudden halt to speculative capital movements after a short period of time has resulted in a greater number of solutions being feasible compared with the situation in which speculation was allowed to continue unabated. of particular interest are the solutions to SPEC 22, SPEC 24, SPEC 34 and SPEC 35 in which the deterministic solutions are clearly infeasible due to the nature of the required time paths of the controls, whereas the stochastic solutions are feasible. Once again we have a situation in which general additive uncertainty has been complementary to uncertainty resulting from unanticipated speculation in relation to requiring a less severe monetary policy than the policy which prevailed in the absence of additive shocks. This type of counterintuitive result has been a feature of many experiments carried out in this study and supports the more generalised theoretical work already cited. It is clear that uncertainty need not necessarily be a hindrance to policymakers or be destabilising.

The ability of the system to get back on target is reflected in Figures 42 and 43 where selected experiments have been graphed. The graphs of SPEC 17, SPEC 29, SPEC 15 and SPEC 27 reveal a rapid shift back to the original optimal path without speculation and this behaviour of the target variables was reflected in all of the cut-off experiments and yields a minimal welfare loss from the non-speculative case. Thus, a brief unanticipated speculative capital movement in capital flows takes on the characteristics of a brief perturbation to the system after which the system is able to quickly recover and return exactly to its desired path. This response was not forthcoming when speculation was allowed to continue unhindered. While the target variables return to the original optimal path, the controls do not. In fact, the presence of a short burst of speculation when monetary targets are incorporated in the cost function 


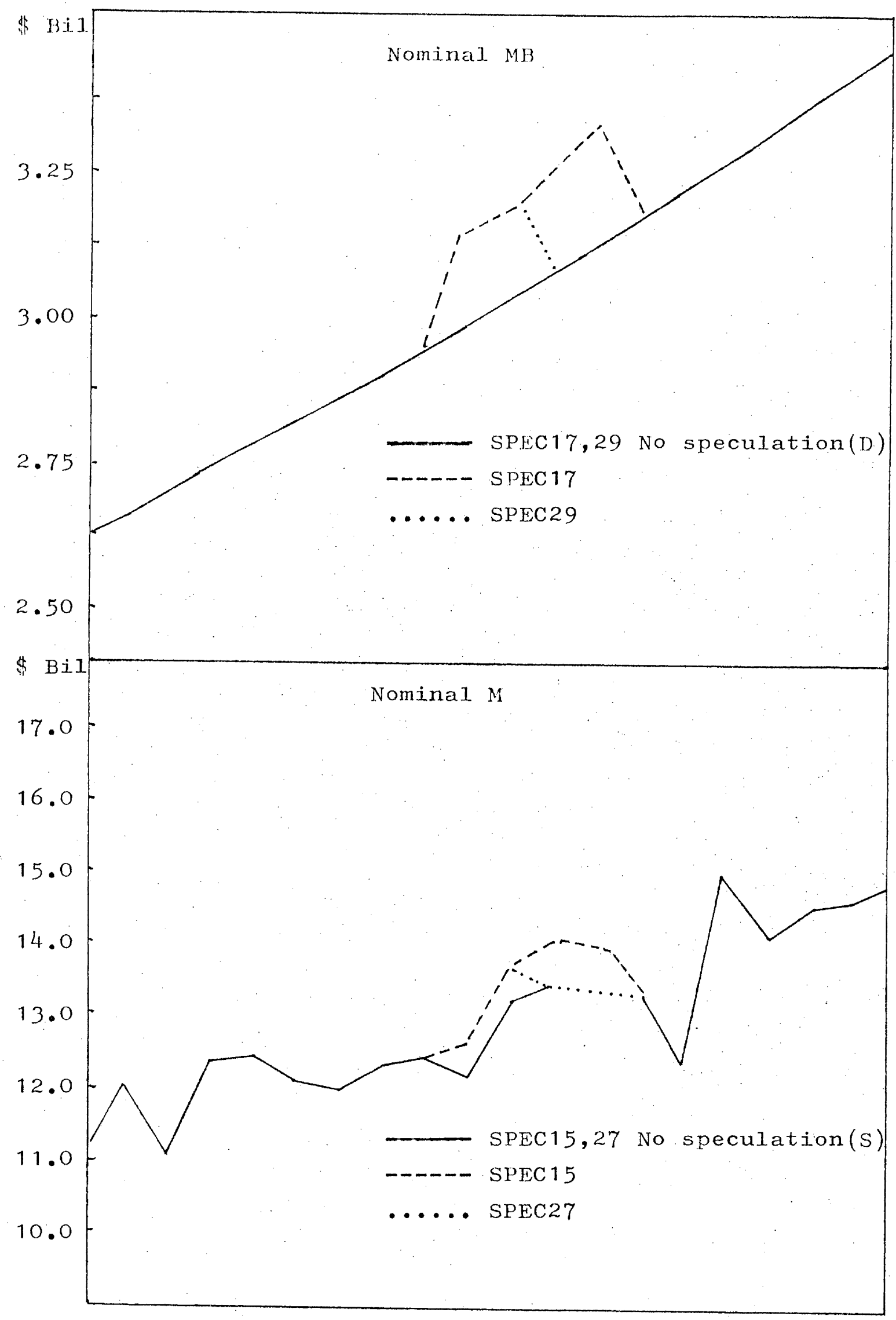

FIGURE 42. 


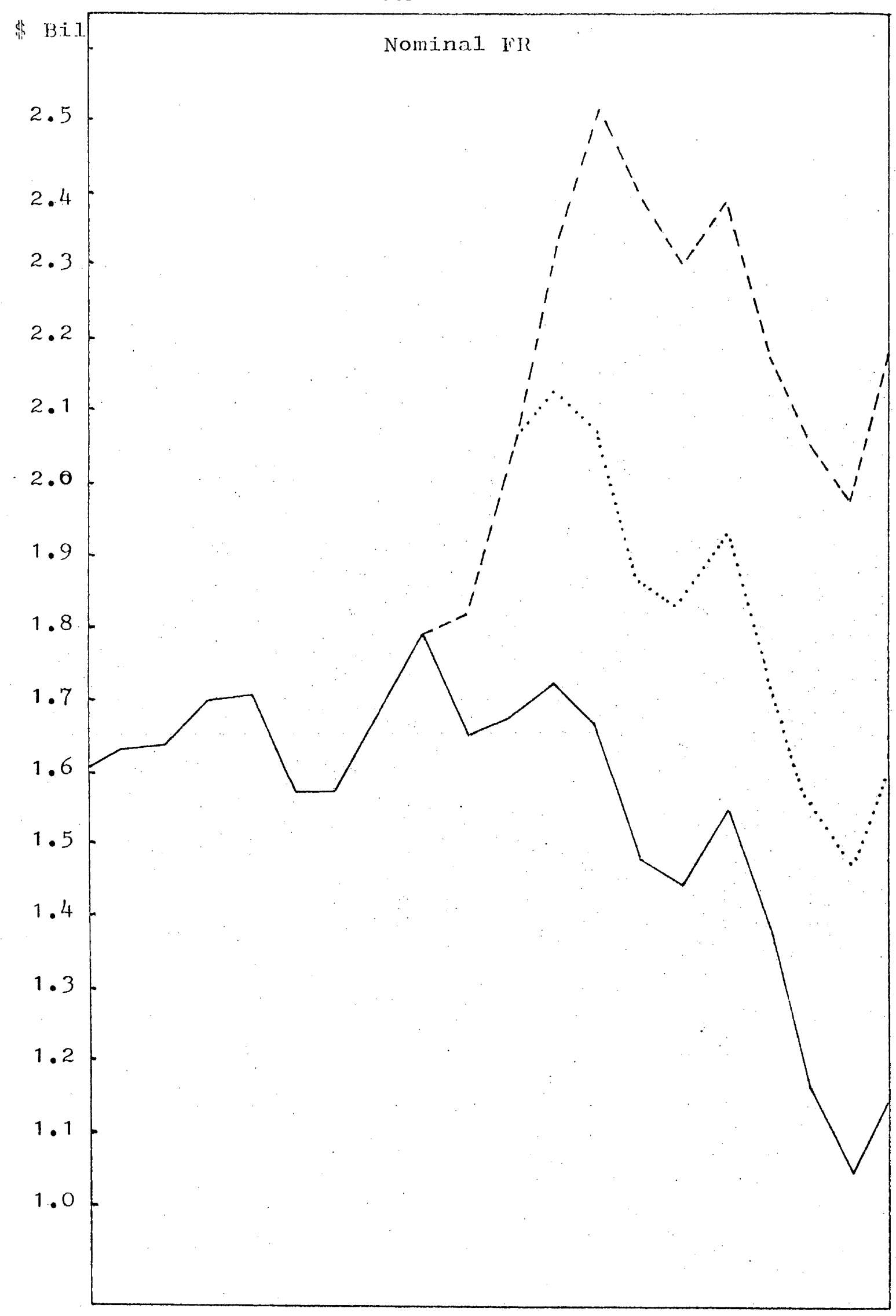

SPEC15,27 No speculation (S)

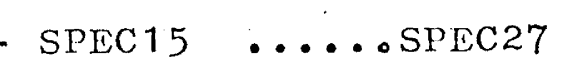

F IGURE 43. 
forces both fiscal and monetary policy to vary from the original optimal path after the target variables have recovered. Similar behaviour can be found in the non-target endogenous variables, particularly in the various time paths for foreign reserves which depart drastically from the nonspeculation case. However, the general behaviour of FR remains identical to the non-speculative case but at a significantly different level. See Figure 43. The failure of $F R$ to get back to the original optimal path was reflected in all experiments as was the tendency for $F R$ to reflect the exact cycles and downturns of the non-speculative case after the initial perturbation. The behaviour of FR has a significant effect on the adjustment of both controls when monetary targets are aimed for (with or without income targets) due to the significance of $\mathrm{FR}_{\mathrm{t}-1}$ in the relevant feedback matrices. See Figures 44 and 45 . The importance of the feedback effects from FR reflects the underlying structural relationship between $F R$ and the monetary sector. In contrast, the inclusion of $\mathrm{y}$ as the sole target in the cost function produces a situation in which the time paths for both controls track very close to the non-speculative optimal paths with both controls returning exactly to the non-speculative time paths by the end of the planning period. This of course reflects the fact that monetary effects arising through balance of payments disturbances are minimised on the income sector due to the size of the financial wealth effect in the consumption function.

The analysis of this chapter yields some important results relating to the use of quantitative stabilisation techniques. Firstly, as we have already mentioned, the fixed target case would appear to be inappropriate when a fixed exchange rate regime is operating. The exception to this being strongly-Tinbergen situations such as IEB2 where reserves can be exactly controlled for all time periods of a planning period. 


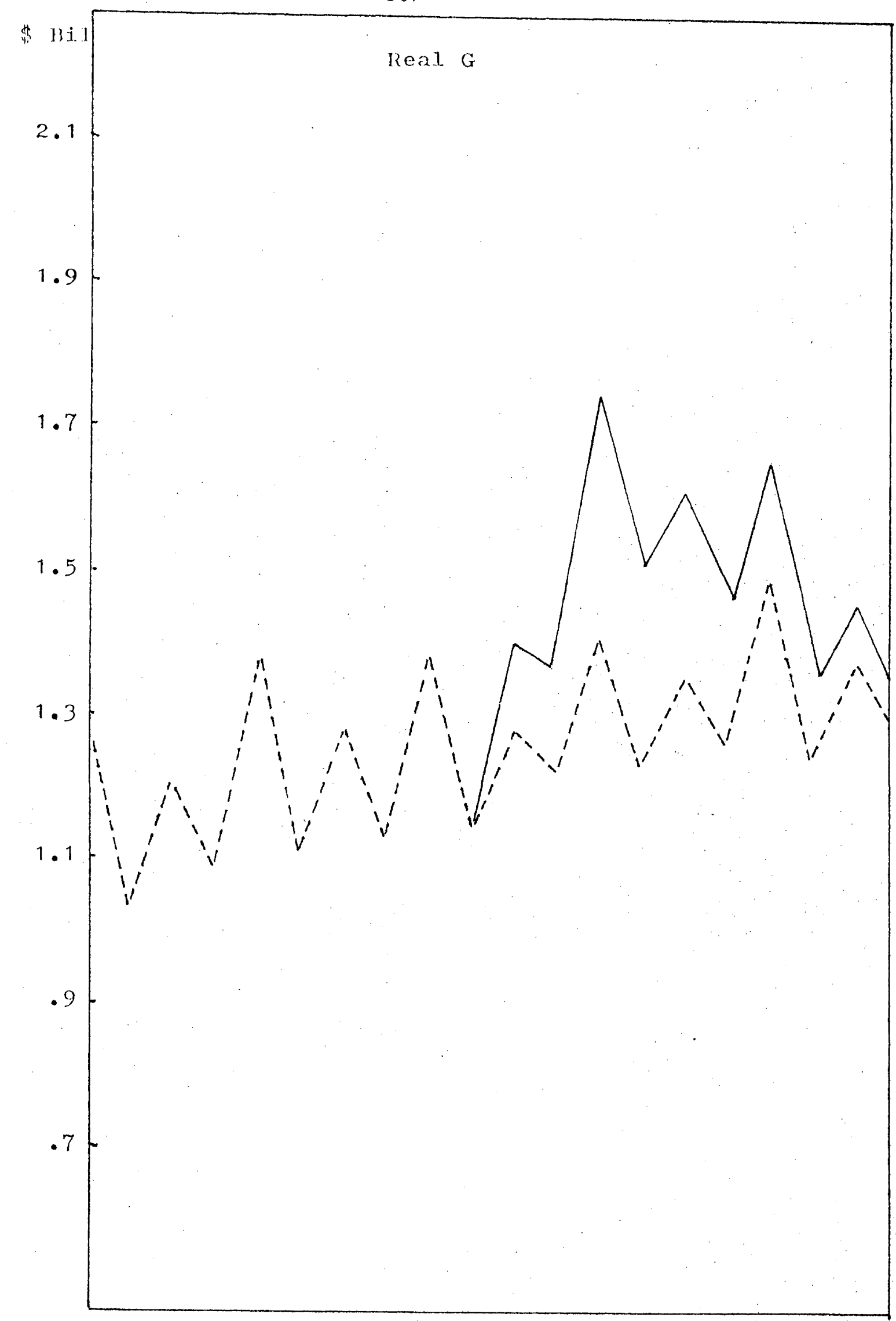

- - - SPEC17(D) No speculation

$\longrightarrow \operatorname{SPEC} 17(\mathrm{D})$ Speculation 


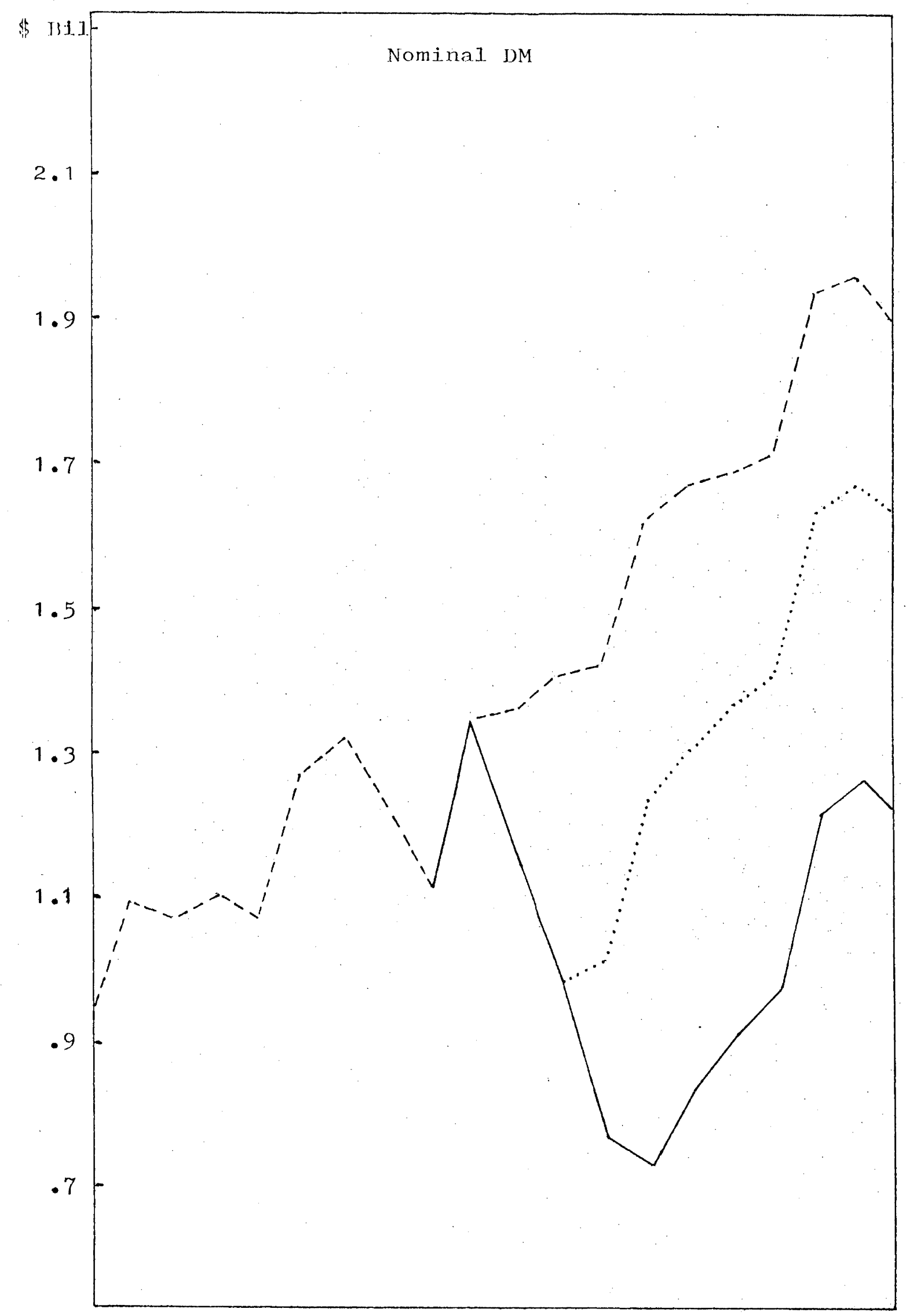

SPEC17,29 No speculation

SPEC17 speculation ......SPEC29 speculation FIGURE 45 . 
Secondly, the treatment of unanticipated speculative capital flows as an additive type shock which cannot be adequately foreseen, will render linear/quadratic techniques ineffective if the speculation is allowed to continue unhindered unless pure income targets are aimed for. At the simulation level, linear/quadratic techniques become feasible and exceptionally powerful when the speculation ceases after a brief period. Indeed, if $\mathrm{Y}$ is the only target then roplanning may not be necessary. At the practical level problems could arise out of the need to plan optimally in advance of the planning period or time period in which policy is to be implemented. (See Chapter 2). This would be especially so in the case of fiscal policy. A condition for the successful implementation of linear/quadratic techniques in a situation of unanticipated speculative capital flows and monetary targets is that a major restructuring of policy implementation takes place which would allow all instruments to be adjusted rapidly without being subject to the bureaucratic and political inertia to which they are subject at the present time. Monetary instruments are generally more flexible in practice than government spending with respect to rapid adjustment to immediate past states of the system and as monetary policy is the major weapon against balance of payments perturbations (in the absence of a freely adjusting exchange rate), the inability of fiscal policy to instantaneously adjust (instantaneously implemented) may not be a hindrance in all cases. The previous experiments suggest that in some cases a rapid adjustment by $G_{t}$ (within the unit time period) in response to immediate past speculative influences will be required but only in a minority of situations depending largely on the relative weights in the cost function. The analysis does suggest that in general a more appropriate model in relation to the use of monetary instruments and the monetary and open 
sectors of an economy would perhaps be monthly rather than quarterly. This conclusion has arisen elsewhere in this study and illustrates an important constraint on the use of quantitative techniques for economic stabilisation. As the correct income model, given the present level of sophistication in relation to data collection, is likely to be quarterly, the use of a monethly monetary model would require some decentralisation and deterioration in the coordination and implementation of policy. The results presented in earlier chapters of this study clearly show that policy needs to be coordinated thus some very short run monetary control may have to be sacrificed through the use of a fully integrated model to achieve a reasonable degree of policy coordination. This apparently paradoxical situation requires further attention, particularly if formal stabilisation techniques are to become an integral part of policy formulation and decision making.

The results also suggest that the cessation of speculative movements need not be permanent. Indeed, Figure 43 reveals that speculative movements are likely to be triggered again (perhaps in the opposite direction) thus further complicating the implementation of policy and adding an additional constraint to the practical use of linear/quadratic techniques. In general terms the results indicate the difficulties in implementing formal stabilisation techniques in an open economy with a fixed exchange rate. To avoid such complications a flexible exchange rate could be employed, or, in a linear/quadratic framework, the balance of payments would need to be incorporated in the cost function as a major target subject to allowing instruments to adjust frecly. Alternatively, the treatment of FR as a target in a strongly-Tinbergen framework will also preclude the occurrence of speculation arising from an upswing or downswing in reserves. The results of the previous experiments suggest 
that given the two above situations, even the presence of additive uncertainty in all sectors of the economy will be unlikely to result in unanticipated speculative capital movements. The analysis has assumed perfect forecasting ability in relation to the other uncontrollable exogenous variables of the system. For practical purposes it is doubtful if exact forecasting could be achieved, particularly in relation to exports which form a major component of the open sector. It would appear that a relaxation of the perfect forecasting assumption would necessarily inhibit any extensive planning and in particular would limit the degree of control over the open sector. This in turn could result in a failure to adequately plan and adjust for speculative capital movements. This need not be the case however. For example, in a strongly-Tinbergen framework there is only a need to forecast one period in advance. Assuming that policy can be implemented fairly quickly then one period forecasts would not be an undue hindrance and it could be expected that forecast exrors would be relatively small in most cases thus allowing for close control of the targets. The linear/quadratic approach can be modified by using learning techniques or by adjusting the length of the planning period until it is felt that a reasonably accurate forecast can be made. Unfortunately the need to plan in advance will tend to make forecasts more inaccurate but at least a reduction in the length of the planning period will reduce the degree of forecast error in the absence of any severe abnormal external shocks. 


\section{CHAPTER IX}

\section{CONCLUSION AND SUMMARY}

The major conclusions of this study have been discussed in detail at the end of each relevant chapter but it is nonetheless useful to reiterate the major points. Firstly, it is quite clear that given the model and the target levels for specific endogenous variables, the dynamic Tinbergen or fixed target approach to stabilisation is vastly inferior to an optimisation or flexible target approach. This result does appear at first sight to be counter-intuitive as many policy-makers would agree that the underlying philosophy of the fixed target approach is more appropriate to stabilising the economy than an optimising approach. It is not uncommon to hear both politicians and policy-makers arguing that any specified targets can only be reached after a degree of time has passed (the policy lead or anticipation period in the fixed target framework). The results appear to concur with this view as it has been clearly shown that if the equality between instruments and targets is broken and if the number of targets exceeds the number of available instruments, optimisation will result in the non-achievement of all targets. However, it has also been clearly shown that to exactly achieve all targets by an appropriate anticipation of the target path, requires infeasible policy prescriptions, even if only a relatively small gap is to be closed between the target and a corresponding optimal solution. In fact, in an open economy, the achievement of targets selected from different sectors of the economy will result in severe policy adjustments even in a strongly-Tinbergen framework. This of course is due to the way in which the sectors interrelate and the experiments of the previous chapters indicate how action to stabilise one sector of the cconomy can create adverse movements in another sector. 
The results do not necessarily indicate that it is wrong to try and anticipate a target path objective as it must be remembered that it has been implicitly assumed that the model is the correct model. This has allowed exact fine tuning at the simulation level. At the practical level policy-makers may not have such a profound faith in the properties of any econometric model and would most likely use a combination of judgemental and quantitative analysis to arrive at a suitable policy sequence. This factor combined with a tendency for the structure of the economy to evolve over time would most likely mean that it would be deemed satisfactory to steer the economy to within reasonable bounds of the targets after a suitable anticipation period. It should be stressed that the results obtained in this study are only meant to be indicative of what could happen if either of the two stabilisation techniques were implemented. Unfortunately, our knowledge of control techniques has tended to exceed our knowledge of how the economy works. If, in the future, policy-makers are prepared to accept econometric models for actual planning purposes and are willing to implement the policies generated by applying stabilisation techniques to those models, then the conclusions of this study will become particularly relevant. What is required now is considerable additional research on fixed target techniques using alternative models and target sets, as the use of such techniques as a guide for policy-making may not be too far away in the future - policy-makers are already beginning to use optimisation techniques.

The complications of an open economy have been stressed throughout this study. In particular, it is clear that the phasing and implementation of both monetary and fiscal policy needs to be co-ordinated to a high degree. The internal and external balance and monetary experiments illustrate how 
policy manipulations by the fiscal and monetary authorities can impinge on other sectors of the economy producing the need to offset such influences while trying to steer a particular sector towards a designated goal. The successful implementation of a stabilisation strategy requires a high degree of centralised policy-making which in turn requires the use of at least a quarterly model. In fact, it has been argued that as monetary policy is generally easier to quickly implement than fiscal policy, a quarterly model may even be inappropriate for monetary and open sector stabilisation and a degree of control over the money and open sectors may need to be sacrificed in order to achieve policy co-ordination within a quarterly framework. The loss of control over the open and monetary sectors will be more pronounced under significant uncertainty as the monetary instrument will be prevented from adjusting to offset very short-run perturbations. Perhaps what is required to overcome this problem is a quarterly income sector coupled with monthly open and monetary sectors. The only problem here would be trying to model the linkages, particularly if quarterly income data was required.

Uncertainty has been a key issue throughout this study. The presence of uncertainty is of particular importance in relation to the open sector and to a lesser extent in relation to the monetary sector. The internal and external balance experiments of chaptex Five illustrate how additive uncertainty can shift the open sector off target due to the nature of the identity for foreign reserves. This poses a particular problem for external stabilisation and it is clear that if uncertainty is present in the open sector to a considerable extent, then the open sector will need to be heavily weighted in the cost function in an optimisation framework to ensure the exclusive assignment of the monetary instrument. We have seen 
that an exclusive assignment of the monetary instrument to the open sector can keep the time path for foreign reserves very close to i.ts target when uncertainty is present. On another level, we have seen that the presence of uncertainty can produce significant switches in the use of fiscal and monetary policy from the deterministic case. In particular, it has been shown that in some instances the use of policy can be more passive under uncertainty than certainty - a result which many policy-makers may find surprising. The change in the mix of policy from a certainty situation to one of uncertainty suggests that if policy is formulated on the basis of perfect information (which it may be necessary to do) and then implemented in an uncertain world, significant destabilisation may occur. The results of the preceding experiments confirm this to a large extent, although it may be possible in the case of a total monetary base target to successfully implement a deterministic policy in a stochastic world. The results of the internal and external balance experiments also illustrate that it is possible to obtain a greater degree of control with less instruments under uncertainty than can be obtained with additional instruments. This result is very important as it indicates that uncertainty need not be a hindrance to stabilisation as is commonly assumed. The stabilising properties of uncertainty appear throughout this study, particularly in relation to unanticipated speculative capital flows. The possibility of speculative capital flows under a fixed exchange rate regime is a special form of uncertainty which can seriously disrupt any fixed or flexible target strategy. However, by treating speculation as a type of additive uncertainty, minimal disruption to a stabilisation strategy obtained from optimisation can result.

One aim of this study was to compare the stochastic performance of both the fixed and flexible target approaches to stabilisation and to 
examine the loss of fine tuning in the fixed target framework. Optimisation is clearly superior to fixed target stabilisation under uncertainty when the number of natural targets exceeds the number of natural instruments. This of course follows from the feedback nature of the optimal control laws. In a strongly-Tinbergen framework, a fixed target solution is probably marginally superior to optimisation under uncertainty in that the fixed target solution explicitly includes the initial conditions or immediate past state of the system in the solution procedure. On the other hand, an optimisation solution may require the system to settle down if there is a significant divergence between the initial state and the targets. It should not be forgotten that in a strongly-Tinbergen situation, optimisation can be regarded as redundant. Unfortunately, the nature of the fixed target, policy lead solutions, mean that a detailed discussion of the stochastic results will be of little additional value. This is an area in which there is ample scope for additional research with alternative models.

The presentation of particular feedback matrices in Chapters Five and Six not only indicates the difficulties in trying to estimate historical preference functions, but also casts some doubt on a comment put forward by Benjamin Friedman (1975a) in the conclusion of his volume on applied stabilisation. Friedman observed that the feedback matrices obtained from optimal experiments could be used to derive rules for alternative scenarios, for example, inflation, recession and so on. Friedman's comment overlooks the fact that if we have a model from which we can obtain rules that we can believe in then of course there is no need to use simple rules - we would do better by using the results of the optimal stabilisation experiments. Leaving this aspect aside, it is clear from the feedback matrices presented in this study that in the short-run, the 
cocfficients of the feedback matrices change so often over a planning horizon that no single rule could be isolated. Further complications are introduced by the behaviour of the additive vector which is liable to shift dramatically from period to period and be an important factor in the formulation of optimal controls. Simple rules of the type considered in the rules versus discretion controversy which originated with Simons (1936) do not usually contain a constant term and if it is desired to include a constant term, it is clear from the results that there is no way of specifying a single, appropriate number based on the optimal solutions.

A number of issues relevant to the applied control theorist have also been raised, the most important being the notion of cost function bias. It is only be eliminating cost function bias that exact trade-offs can be maintained and legitimate and meaningful welfare comparisons can be made between alternative cost function specifications. A technique for deriving target paths for seasonally unadjusted variables was introduced and implemented and it was recognised that in the formulation of appropriate target paths, the consistent specification of identities does not guarantee the consistent achievement of the components of the identity or the variable described by the identity. The above issues have been overlooked by applied control theorists in the past but should be considered when stabilisation scenarios are being formulated and executed.

While optimisation techniques have received a good deal of attention in relation to economic stabilisation, there still remains a considerable area for research into alternative stabilisation techniques such as the dynamic Tinbergen fixed target approach. The present study only touches the surface. Additional work needs to be carried out in the stochastic area and it would be beneficial to compare the sensitivity of flexible and fixed target techniques to coefficient and more general 
structural adjustments, sensitivity to forecast errors in the uncontrollable exogenous variables and the initial conditions. In fact, little or no attention has been paid to forecasting exrors in relation to exogenous variables and initial conditions with respect to optimal control, so this gap needs to be filled before the fixed target approach is analysed in detail. The results of this study present a fairly gloomy picture of the fixed target approach to stabilisation but as this constitutes the first attempt at an applied analysis of such problems, the results should not be taken as the final conclusive evidence on the subject. Alternative models and target sets, particularly target sets which only include variables from one sector of the economy, may yield feasible results which may allow the fixed target approach to be regarded as a feasible and perhaps more acceptable alternative to optimal macroeconomic control. 


\section{FOOTNOTES}

1 An example of a non-linear technique which provides a solution in which it is not clear if it would be any better in terms of computational costs or accuracy of the algorithm in relation to obtaining an optimal solution than a linear treatment of the same problem can be found in the work of Gupta et al (1975). There is some uncertainty about the accuracy of the technique (page 266) "While it cannot be proven that the algorithm converges to the global optimum, the probability of such convergence can be shown to increase with the number of initial points randomly chosen in the solution space...Thus it is likely that the policy solutions we present are globally optimal". There is however, no guarantee that this is the case and would depend on the number of initial points chosen.

2 The Theil-Tinbergen procedure (Theil (1964), Tinbergen (1952)) can also be employed which obviates the need for a large state space realisation, see Friedman (1975a). The choice of technique is usually made on the grounds of convenience or solution tractability and the preferences of the researcher. In any case, Norman (1976) has recently shown that the dynamic programming technique and the Theil-Tinbergen approach are in. fact logically equivalent.

3. This is a standard mathematical result. See Chow (1970) for a further analysis in relation to optimal control analysis.

4 A more detailed discussion, although within slightly modified frameworks, can be found in the works of Garbade and Preston cited above. Garbade employs a Chow type framework while Preston utilises the conventional control theory formulation which as yet has not gained a wide acceptance in the economic control theory literature. In addition, Preston relies on the control concepts of observability and detectability, concepts which have been part of the control literature for some time but have been ignored in economic applications. Nevertheless, it is possible to summarise the major results in a succinct manner and at the same time seeing intuitively why certain conditions are applicable, without resorting to a prolonged rigorous analysis.

5 While the specification of $R_{t}>0$ is not necessary for policy uniqueness in the dynamic programming framework presented here, $R_{t}>0$ is necessary in the minimum Principle solution used by Pindyck (1973) where $R_{t}{ }^{-1}$ appears as an individual component of the solution. The dynamic programming procedure is generally more flexible then, although $\mathrm{R}_{t}=0$ can be approximated in the minimum Principle framework by setting $\mathrm{R}_{t}^{t}>0$ with the weights extremely small in relation to the output weights. 
6 I am indebted to a number of public servants for contributing some revealing insights into some of these aspects. They, of course, must remain anonymous.

$7 \bar{A}, \bar{C}$ and $\bar{D}$ denote structural coefficients as distinct from reduced form coefficients.

8 The relationship between the final form and the state space form was initially established by Preston and Wall (1973).

9 B must be mxm. If $B$ is greater than mxm then appropriate adjustments can be made by deleting time-indexed instruments. This is discussed elsewhere in Chapter Two.

10 (2.64a) illustrates that $s$ is not completely arbitrary but that there exists a minimal policy horizon, the length depending on the number of targets and instruments.

11 Turnovsky (1968) has successfully employed this hypothesis in an applied analysis of New Zealand imports.

12 After this study was completed the author became aware of a later study by Porter, Porter (1976), in which the domestic assets of the Reserve Bank (DM) were broken up into constituent parts giving an overall better fit to the data. It would appear that the improvement in fit would not substantially alter the general nature of the results.

13 We have already noted that this is extremely difficult in Australia due to the institutional nature of wage and price determination.

14 See Kareken, Muench and Wallace (1973) for a detailed discussion of some important problems associated with this issue.

15 The analysis of the problem is carried out in Turnovsky's paper with an infinite time horizon although it is indicated that a similar set of results can be obtained with a finite time horizon. Turnovsky's analysis also allows for the case where the number of targets is greatex than the number of instruments which is more general than Holbrook's original framework. 
16 While no formal dynamic stability analysis has been carried out, an examination of the reduced form indicates that we could reasonably expect $Y$ to be a stable function based on the rule of thumb technique of examining the size of the coefficients of the lagged endogenous variables, particularly lagged $Y$. Simulation studies with both instrument constant lend support to this assertion and similarly help confirm that no oscillatory behaviour in $\mathrm{Y}$ occurs over the twenty period policy interval.

17 At the present time an exhaustive investigation into instrument instability in the fixed target framework is being conducted by Preston and Sieper at the Australian National University.

18 The disturbances were generated in a manner which produced zero auto-correlation. This procedure eliminated the computational complication of using control laws which would take account of the auto-correlation. For practical planning purposes any autocorrelation present in the model could be accounted for by implementing an appropriate control technique, for example, the technique outlined by Pagan (1975).

19 The terms "strong", "expansionary" and "contractionary" are used in relation to the position of the optimal policy path relative to its target. Where stochastic and deterministic results are being compared, the terms refex to the relative position between those two paths.

20 The comparison is made in terms of the deterministic results as the historical data used to estimate the model will contain any historical shocks and the historical fiscal and monetary policies will contain adjustments for those shocks, that is, only if the shocks were detected sufficiently quickly enough to enable counteractive policy action.

21 Several other exploratory experiments were carried out with different targets which indicated that a severe divergence between initial conditions and targets required that the solution settle down before the targets were achieved exactly.

22. The feedback matrices for IEB2 are identical for all time periods and are largely redundant given that the problem can be solved within a strongly-Tinbergen framework and as such, have not been included.

23 The same result could occur when zero costs are allocated to the instruments. 
24 Policy planners could perhaps adjust relatively easily to monetary disturbances where the data is often of a monthly or even weekly nature but could not adjust easily to lagged income say, where there are very real delays in obtaining the correct information about the previous level or current level of $Y$.

25 The length of lags in the system is also important as lags greater than say three periods, would probably allow all instruments to adjust in response to past shocks in the state vector. Not so however for one period lags.

26 For a discussion of related issues concerning this problem see Friedman (1975b).

27 It could be argued that the model used here is too simple for any such conclusions to be drawn. However, in terms of complexity it is certainly no worse than a considerable number of monetarist models which have been used for theoretical analysis and applied work, for example, the model of Andersen and Jordan (1970).

28. Evidence of this appeared in Australia in recent years, particularly up to 1972, with attempts to keep interest rates stabilised being consistent with a rapidly expanding total monetary base and money supply with annual increases of $25 \%$ in nominal $M$ not being uncommon.

29 A similar conclusion holds for the case where the supply of money and the rate of interest are exactly controlled.

30 It should not be forgotten that the structural properties of the system will not change and the instrument will still be structurally unstable, which should be distinguished from stability at the implementation level.

31 This effect was also apparent in the IEB experiments. The scaling of weights is not so important in the monetary experiments as only one target is aimed for. Note also that the auxiliary state variables are also important components of the feedback matrices which places some doubt on the historical estimation of feedback matrices unless the exact, true model is used.

32 Further monte carlo experiments were carried out which confirmed the robust nature of the lincar/quadratic solution. In cach case the target of concern was stabilised around its desired time path. 
33 Strongly-Tinbergen experiments were carried out where there were two targets and both instruments were allowed to adjust freely (a weight of $\cdot 1$ in an optimising framework). The results are interchangeable.

34 A small exchange rate adjustment would only have a minimal effect on other areas of the economy, for example the price level, and would not change the general results. 


\section{BIBLIOGRAPHY}

Abel, A.B. (1975), A comparison of three control algorithms as applied to the monetarist-fiscalist debate, Annals of Economic and Social Measurement, vol.4, Spring, 239-252.

Adams, C. and M.G. Porter, (1976), The stability of money demand-Australia 1965-75, Working Paper, Australian National University.

Andersen, L.C. and K.M. Carlson, (1970), A monetarist model for economic stabilization, Federal Reserve Bank of St Louis Review, vol.52, April, 7-25.

Andersen, L.C. and J.L. Jordan (1968), Monetary and fiscal actions: A test of their relative importance, Federal Reserve Bank of St Louis Review, vol.50, November, 11-24.

Ando, A. and F. Modigliani, (1963), The life-cycle hypothesis of saving: Aggregate implications and tests, American Economic Review, vol.53, March, 55-84.

Ando, A. and C. Palash, (1976), Some stabilization problems of 1971-75 with an application of optimal control algorithms, American Economic Review, vol. 66, May, 346-348.

Aoki, M. (1967), Optimization of Stochastic Systems, N.Y.: Academic Press.

Aoki, M. (1976), Optimal Control and System Theory in Dynamic Economic Analysis, Amsterdam: North-Holland Publishing Company:

Athans, M. (1966), The status of optimal control theory and applications for deterministic systems, IEEE Transactions on Automatic Control, vol,1l, July, 580-596.

Athans, M. (1972), The discrete time linear-quadratic-Gaussian stochastic control problem, Annals of Economic and Social Measurement, vol.1, October, 449-492.

Athans, M. (1974), The importance of Kalman filtering methods for economic systems, Annals of Economic and Social Measurement, vol. 3, January, 49-64. 
Athans, M. and G.C. Chow, (1972), Introduction to stochastic control theory and economic systems, Annals of Economic and Social Measurement, vol. 1, October, 375-384.

Athans, M. and P. Falb (1966), Optimal-Control - An Introduction to the Theory and its Applications, N.Y.: McGraw-Hill Book Company.

Baumol, W.J. (1961), Pitfalls in contracyclical policies: Some tools and results, Review of Economics and Statistics, vol. 43, February, $21-26$.

Baumol, W.J. (1970), Economic Dynamics, Third Edition, N.Y.: McMillan.

Bellman, R.E. (1957), Dynamic Programming, Princeton: Princeton University Press.

Bellman, R.E. and S.E. Dreyfus, (1962), Applied Dynamic Programming, Princeton: Princeton University Press.

Benavie, A. (1972), Mathematical Techniques for Economic Analysis, New Jersey: Prentice-Hall Inc.

Blinder, A. and S. Goldfeld, (1972), Some implications of endogenous stabilization policy, Brookings Papers on Economic Activity, Part 3, 585-640.

Boddy, R. and J. Crotty (1975), Macro-policy goals in the postwar period: Comment, Quarterly Joumal of Economics, vol. 89, November, 673-676.

Brainard, W. (1967), Uncertainty and the effectiveness of policy, American Economic Review, vol. 57, May, 411-433.

Branson, W.H. (1972), Macroeconomic Theory and Policy, N.Y.: Harper and Row.

Bray, J. (1974), Predictive control of a stochastic model of the U.K. economy simulating present policy making practice by the U.K. government, Annals of Economic and Social Measurement, vol. 3, January, 220-251.

Brunner, K. (ed.) (1969), I'argets and Indicators of Monelary Policy, San Francisco: Chandler Printing Company. 
Bryson, A.E. and Y. Ho, (1969), Applied Optimal Control: Optimization, Estimation and Control, Waltham: Blaisdell Publishing Company .

Canon, M.D., C.D Cullum, and E. Polak (1970), Theory of Optimal Control and Mathematical Programming, N.Y.: MCGraw-Hill Book Company.

Chow, G.C. (1970), Optimal stochastic control of linear economic systems, Journal of Money, Credit and Banking, vol. 2, August, 291-302.

Chow, G.C. (1972a), How much could be gained by optimal stochastic policies?, Annals of Economic and Social Measurement, vol. 1, October, $391-406$.

Chow, G.C. (1972b), Optimal control of linear econometric systems with finite time horizon, Intemational Economic Review, vol. 13, February, 16-25.

Chow, G.C. (1973a), Effect of uncertainty on optimal control policies, Intemational Economic Review, vol. 14, October, 632-645.

Chow, G.C. (1973b), Problems of economic policy from the viewpoint of optimal control, American Economic Review, vol. 43, December, $825-837$.

Chow, G.C. (1975a), Analysis and Control of Dynamic Economic Systems, N.Y.: Wiley-Interscience.

Chow, G.C. (1975b), A solution to optimal control of linear systems with unknown parameters, The Review of Economics and Statistics, vol. 57, August, 338-345.

Chow, G.C. (1976a), An approach to the feedback control of non-linear econometric systems, Annals of Economic and Social Measurement, vol. 5, Spring, 297-309.

Chow, G.C. (1976b), Control methods for macroeconomic policy analysis, American Economic Review, vol. 66, May, 340-345.

Chow, G.C. (1.976C), The control of non-linear econometric systems with unknown parameters, Econometrica, vol. 44, July, 685-695. 
Citron, S.J. (1969), Elements of Optimal Control, N.Y.: Holt, Rinehart and Winston.

Cooper, J.P. and S. Fischer, (1972), Stabilization policy and lags: summary and extension, Annals of Economic and Social Measurement, vol. 1, October, 407-418.

Cornwall, J. (1965), The structure of fiscal models, Quarterly Journal of Economics, vol. 79, November, 608-622.

Craine, R., H. Haverner and P.Tinsley (1976), Optimal macroeconomic control policies, Annals of Economic and Social Measurement, vol. 5, Spring, 191-203.

Crotty, J. (1973), Specification error in macro-econometric models: the influence of policy goals, American Economic Review, vol. 63, December, 825-837.

Crotty, J. (1976), Specification error in macro-econometric models: the influence of policy goals-reply, American Economic Review, vol. 66, September, 665-667.

Culbertson, J.M. (1968), Macroeconomic Theory and Stabilization Policy, N.Y.: MCGraw-Hill Book Company.

Dorfman, R. (1969), An economic interpretation of optimal control, American Economic Review, vol. 59, December, 817-831.

Duchan, A.I. (1974), A clarification and a new proof of the certainty equivalence theorem, International Economic Review, vol: 15, February, 216-224.

Duesenberry, J.S., G. Fromm, L.R. Klein and Kuh,E. (eds.) (1965), The Brookings Quarterly Econometric Model of the United States, Amsterdam: North-Holland Publishing Company.

Duesenberry, J.S., G. Fromm, L.R. Klein, and E. Kuh (eds.) (1969), The Brookings Model: Some Further Results, Amsterdam: NorthHolland Publishing Company.

Evans, M.K. and L.R. Klein, (1968), The Wharton Econometric Forecasting Model, Second Edition, Philadelphia: University of Pennsylvania. 
Fischer, S. and J.P. Cooper, (1973), Stabilization policy and lags, Journal of Political Economy, vol. 81, May/June, 847-877.

Fitzgerald, V.W., H.N.Johnston and A.J. Bayes (1973), An interactive computing algorithm for optimal policy selection with nonlinear econometric models, Australian Bureau of Statistics.

Fox, K., J. Sengupta and E. Thorbecke, (1966), The Theory of Quantitative Economic Policy, Amsterdam: North-Holland Publishing Company.

Friedlaender, A.F. (1973), Macro-policy goals in the postwar period: a study in revealed preferences, Quarterly. Journal of Economics, vol. 87, February, 25-43.

Friedman, B.M. (1972), Optimal economic stabilization: an extended framework, Journal of Political Economy, vol. 80, september/ October, 1002-1022.

Friedman, B.M. (1975a), Economic Stabilization Policy: Methods in Optimization, Amsterdam: North-Holland Publishing Company.

Friedman, B.M. (1975b), Targets, instruments and indicators of monetary policy, Joumal of Monetary Economics, vol. 1, October, $443-473$.

Friedman, B.M. (1976), A note on uncertainties in control applications, Annals of Economic and Social Measurement, vol. 5, Spring, 223-224.

Friedman, M. (1968), The goals and criteria of monetary policy, in W. Hamovitch (ed), Monetary Policy: The Argument from Keynes' Treatise to Friedman, Massachusetts: D.C. Heath and Company.

Friedman, M. (1968), The Role of Monetary Policy, American Economic Review, vol. 58, March, 1-17.

Fromm, G. (1976), Applications of optimal control to problems of economic stabilization - discussion, American Economic Review, vol.66, May, 359-360.

Fromm, G. and P. Taubman, (1968), Policy Simulations with an Econometric Model, Washington: The Brookings Institution. 
Gandolfo, G. (1972), Mathematical Methods and Models in Economic Dynamics, Amsterdam: North-Holland Publishing Company.

Garbade, K.D. (1975a), Discretionay Control of Aggregate Economic Activity, Massachusetts: Lexington Books.

Garbade, K.D. (1975b), Discretion in the choise of macroeconomic policies, Annals of Economic and Social Measurement, vol. 4, spring, 215-238.

Garbade, K.D. (1976), on the existence and uniqueness of solutions to multi-period linear/quadratic optimal control problems, International Economic Review, vol. 17, October, 719-731.

Goldfeld, S.M. (1973), The demand for money revisited, Brookings Papers on Economic Activity, vol. 3, 577-646.

Goldfeld, S.M. (1976), Specification exror in macro-econometric models: the influence of policy goals-comment, American Economic Review, vol. 66, September, 662-664.

Gramlich, E.M. (1971), The usefulness of monetary and fiscal policy as discretionary stabilization tools, Journal of Money, Credit and Banking, vol. 3, May, 506-532.

Gregory, R.G. and I.D.Martin, (1976), An analysis of relationships between import flows to Australia and recent exchange rate and tariff charges, Economic Record, vol. 52, March, 1-25.

Gupta, S.K., L.H. Meyer, F.Q. Raines and T. Tarn, (197.5), Optimal co-ordination of aggregate stabilization policy and price controls: some simulation results, Annals of Economic and Social Measurement, vol. 4, Spring, 253-270.

Hansen, B. (1958), The Economic Theory of Fiscal Policy, London: Allen and Unwin.

Hansen, B. (1968), Lectures in Economic Theory II: The Theory of Economic Policy and Planning, Lund: Studentlitteratur.

Heller, H.R. (1965), The demand for money: the evidence from the shortrun data, Quarterly Journal of Economics, vol. 79, May, 291-303. 
Helliwell, J.F. (1969), Monetary and fiscal policies for an open economy, Oxford Economic Popers, vol. 21, March, 35-55.

Higgins, C.I. and.V.W. Fitzgerald, (1973), An econometric model of the Australian economy, Joumal of Econometrics, vol. 1, 229-265.

Higgins, C.I., H.N. Johnston and P.L.Coghlan (1976), Business investment: the recent experience, mimeograph, Australian Bureau of Statistics and Australian Treasury.

Holbrook, R.S. (1972), Optimal economic policy and the problem of instrument instability, American Economic Review, vol. 62, March, 57-65.

Holt, C.C. (1962), Linear decision rules for economic stabilization and growth, Quarterly Joumal of Economics, vol. 76, February, $20-45$.

Howrey, E.P. (1967), Stabilization policy in linear stochastic systems, Review of Economics and Statistics, vol. 49, August, 404-411.

Intrilligator, M.D. (1971), Mathematical Optimization and Economic Theory, New Jersey: Prentice-Hall.

Johnston, J. (1972), Econometric Methods, N.Y.: McGraw-Hill

Kalchbrenner, J.H. and P.A. Tinsley (1976), On the use of feedback control in the design of aggregate monetary policy, American Economic Review, vol.66, May, 349-355.

Kalman, R. E. (1960a), Contributions to the theory of optimal control, Sociedad Matematica Mexicana Boletin, Segunda Série, vol. 5, April, 102-119.

Kalman,R.E. (1960b), On the general theory of control systems, in Proceedings of the First International. Congress of the International Federation of Automatic Control, vol.1, London: Butterworth's.

Kareken, J.H. (1970), The optimum monetary instrument variable, Journal of Money, Credit and Banking, vol. 2, August, 385-390. 
Kareken, J.H., T. Muench, and N. Wallace (1973), Optimal open market strategy: the use of information variables, American Economic Review, vol. 63, March, 156-172.

Kendrick, D. (1976), Applications of control theory to macroeconomics, Annals of Economic and Social Measurement, vol. 5, Spring, $171-190$.

Kendrick, D. and J. Majors (1974), Stochastic control with uncertain macroeconomic parameters, Automatica, vol. 10, 587-594.

Keynes, J.M. (1936), The General Theory of Employment Interest and Money, London: Macmillan.

Kmenta, J.(1966), An econometric model of Australia: 1948-1961, Australian Economic Papers, vol. 5, December, 131-163.

Kmenta,.J.(1971), Elements of Econometrics, N.Y.: Macmillan.

Laidler, D. (1966), The rate of interest and the demand for money - some empirical evidence, Joumal of Political Economy, vol.74, December, 543-555.

Lee, T.H. (1967), Alternative interest rates and the demand for money: the empirical evidence, American Economic Review, vol. 57, December, 1168-1181.

Leijonhufvud, A. (1968), On Keynesian Economics and the Economics of Keynes, London: Oxford University Press.

Livesey, D.A. (1971), Optimising short-term economic policy, Economic Journal, vol. 81, September, 525-546.

Livesey, D.A. (1973), Can macro-economic planning problems ever be treated as a quadratic regulator problem?, IFAC/IFORS International Conference on Dynamic Modelling and Control of National Economies, July, 1-14.

Lutz,F.A. (1968), The Theory of Interest, 2nd Edition, zurich: Verlag.

Mackrell, N.C. (1970), Equations for Tax Payments, Sydney: Reserve Bank of Australia Occasional Paper No. 3c. 
Macrae, E.C. (1972), Linear decision with experimentation, Annals of Economic and Social Measurement, vol. 1, October, 437-448.

Macrae, E.C. (1975), An adaptive learning rule for multi-pcriod decision problems, Econometrica, vol. 5-6, Sept./Nov.,893-906.

Meier, R.C., W.T. Newell and H.L. Pazer, (1970), Simulation in Business and Economics, New Jersey: Prentice-Hall inc.

Moore, B.J. (1972), Optimal monetary policy, Economic Joumal, vol. 82, March, 116-138.

Mundell, R.A. (1962), The appropriate use of monetary and fiscal policy for internal and external stability, IMF Staff Papers, vol. 9, March, 70-77.

Nagar, A.L. (1969), Stochastic simulation of the Brookings econometric model, in J.S. Duesenberry, G. From, L.R. Klein and E.Kuh (eds) The Brookings Model: Some Further Results, Amsterdam: North-Holland Publishing Company.

Naylor, T. (1966), Computer Simulation Experiments with Models of Economic Systems, N.Y.: John Wiley and Sons Inc.

Nevile, J.W. (1962), A simple econometric model of the Australian economy, Australian Economic Papers, vol. 1, June, 79-84.

Nevile, J.W. (1970), Fiscal Policy in Australia: Theory and Practice, Melbourne: Cheshire.

Norman, A.L. (1974), on the relationship between linear feedback control and first period certainty equivalence, Intemational Economic Review, vol. 15, February, 209-215.

Norton, W.E. (1973), Some Principles of Economic Policy, Sydney: Reserve Bank of Australia Occasional Paper No. 6.

Norton, W.E., A.M. Cohen and K.M. Sweeney (1970), A Model of the Monetary Sector, Sydney: Reserve Bank of Australia Occasional Paper No. 3D.

Norton, W.E. and J.F. Henderson (1972), A Model of the Australian Liconomy: A Further Report, Sydney: Reserve Bank of Australia Occasional Paper No. 3G. 
Oudet, B.A. (1976), Use of the linear quadratic approach to study the dynamic policy responses of a non-linear model of the French economy, Annals of Economic and Social Measurement, vol. 5, Spring, 205-210.

Pagan, A. (1975), Optimal control of econometric models with autocorrelated disturbance terms, International Economic Review, vol.16, February, 258-263.

Perkins, J.O.N. (1972), Australia in the World Economy, Melbourne: Sun Books.

Peston, M. (1973), Econometrics and control: some general comments, IFAC/IFORS International Conference on Dynamic Modelling and Control of. National Economies, July, 15-30.

Phillips, A.W. (1954), Stabilisation policy in a closed economy, Economic Joumal, vol. 64, June, 290-323.

Phillips, A.W. (1957), Stabilisation policy and the time forms of lagged responses, Economic Joumal, vol. 67, 265-277.

Pierce, J.L. (1969), Some rules for the conduct of monetary policy, in Controlling Monetary Aggregates, Boston: Federal Reserve Bank of Boston.

Pindyck, R.S. (1972a), An application of the linear quadratic tracking problem to economic stabilization policy, IEEE Transactions on Automatic Control, vol. 17, June, 287-300.

Pindyck, R.S. (1972b), Optimal stabilization policies via deterministic control, Annals of Economic and Social Measurement, vol.1, October, 385-390.

Pindyck, R.S. (1973a), Optimal Planning for Economic Stabilization, Amsterdam: North-Holland Publishing Company.

Pindyck, R.S. (1973b), Optimal policies for economic stabilization, Econometrica, vol. 4l, May, 529-560.

Pindyck, R.S. and S.M. Roberts, (1974), Optimal policies for monetary control, Annals of Economic and. Social Measurement, vol. 3, January, 207-237. 
Pindyck, R.S. and S.M. Roberts (1976), Instruments, intermediate targets and monetary control, International Economic Review, vol.17, October, 627-650.

Pitchford, J.D. (1968), An analysis of price movements in Australia, 1947-1968, Australian Economic Papers, vol. 7,-December, $111-135$.

Pitchford, J.D. and S.J. Turnovsky, (1977), Applications of Control Theory to Economic Analysis, Amsterdam: North-Holland Publishing Company.

Pontryagin, L.S., V.G. Boltyanski, R.V. Gamrelidze and E.F. Miskchenko (1962), The Mathematical. Theory of Optimal Processes, N.Y.: John Wiley and Sons Inc.

Poole, W. (1970), Optimal choice of monetary policy instruments in a simple stochastic macro model, Quarterly Joumal of Economics, vol. 84, May.197-216.

Porter, M.G. (1974), The interdependence of monetary policy and capital flows in Australia, Economic Record, vol. 50, March, 1-20.

Porter, M.G. (1976), The Australian capital account, 1965-75, a monetary view, mimeograph, Australian National University.

Prescott, E.C., (1971), Adaptive planning for macroeconomic planning, Western Economic Journal, vol. 9, December, 369-378.

Prescott, E.C. (1972), The multi-period control problem-under uncertainty, Econometrica, vol. 40, November, 1043-1058.

Preston, A.J. (1974), A dynamic generalization of Tinbergen's theory of policy, Review of Economic Studies, vol.41, January, 65-74.

Preston, A.J. (1975), Optimal stabilisation policy with a lagged instrument, in M. Parkin and A.R. Nobay (eds), Contemporary Issues in Economics, Manchester University Press. 
Preston, A.J. (1977), Existence, uniqueness and stability of linear optimal stabilization policies, in J.D. Pitchford and S.J. Turnovsky (eds), Applications of Control Theory to Economic Analysis, Amsterdam: North-Holland Publishing Company .

Preston, A.J. and Sieper, E. (1977), Policy objectives and instrument requirements for a dynamic theory of policy, in J.D. Pitchford and S.J. Turnovsky (eds), Applications of Control Theory to Economic Analysis, Amsterdam: NorthHolland Publishing Company.

Preston, A.J. and K.D. Wall (1973), Some aspects of the use of state space models in econometrics, IFAC/IFORS International Conference on dynamic modelling and Control of National Economies, July, 226-239.

Roberts, B. and D. Schulze, (1973), Modem Mathematics and Economic Analysis, N.Y.: W.W. Norton.

Runcie, N. (1971), Australian Monetary and Fiscal Policy: Selected Readings vol. 1, London: University of London Press.

Sargent, T.J. (1971), The optimum monetary instrument variable in a linear economic model, Canadian Journal of Economics, vol. 4, Feb., 50-60.

Sengupta, J.K. (1970), Optimal stabilization policy with a quadratic criterion function, Review of Economic Studies, vol. 37, January, 127-145.

Shapiro, H. and Holbrook, R.(1970), The choice of optimal intermediate targets, American Economic Review, vol. 60, May, 40-46.

Shell, K. (ed) (1967), Essays on the Theory of Optimal Economic Growth, Cambridge: MIT Press.

Simon, H.A. (1956), Dynamic programming under uncertainty with a quadratic criterion function, Econometrica, vol. 24, January, 74-81.

Simons, H.C. (1936), Rules versus authorities in monetary policy, Journal of Political Economy, vol. 44, February, 1-30. 
Sims, C.A. (1974), Optimal policies for unstable instruments, Annals of Economic and Social Measurement, vol. 3, January, 257-265.

Smyth, D. (1964), Empirical evidence on the acceleration principle, Review of Economic Studies, vol. 31, June, 185-202.

Stanford, J.D. (1973), Money, Banking and Economic Activity, Sydney: John Wiley and Sons Inc.

Takayama, A. (1974); Mathematical Economics, Illinois: The Dryden Press.

Teigen, R.L. (1964), Demand and supply functions for money in the United States: some structural estimates, Econometrica, vol. 32, October, 476-509.

Theil, H. (1956), On the theory of economic policy, American Economic Review, vol. 46, May, 360-366.

Theil, H. (1957), A note on certainty equivalence in dynamic planning, Econometrica, vol. 25, April, 346-349.

Theil, H. (1964), Optimal Decision Rules for Govermment and Industry, Amsterdam: North-Holland Publishing Company.

Theil, H. (1971), Principles of Econometrics, N.Y.: John Wiley and Sons Inc.

Tinbergen, J. (1952), On the Theory of Economic Policy, Amsterdam: NorthHolland Publishing Company.

Tinbergen, J. (1966), Economic Policy, Principles and Design, Amsterdam: North-Holland Publishing Company.

Trivedi, P.K. and J. Rayner (1976), Wage inertia and comparison effects in Australian award wage determination, 1964-74, Mimeograph, Australian National University.

Turnovsky, S.J. (1968), International trading relationships for a small country: the case of New Zealand, Canadian Joumal of Economics, vol.1, November 772-790. 
Turnovsky, S.J. (1970), Empirical evidence on the formation of price expectations, Joumal of the American Statistical Association, vol. 65, December, 1441-1454.

Turnovsky, S.J. (1973), Optimal stabilization policies for deterministic and stochastic linear systems, Review of Economic Studies, vol. 40, January, 79-96,

Turnovsky, S.J. (1974), The stability properties of optimal economic policies, American Economic Review, vol. 64, March, 136-148.

Turnovsky, S.J. (1975), Optimal choice of monetary instruments in a linear economic model with stochastic coefficients, Joumal of Money, Credit and Banking, vol. 7, February, 51-80.

Turnovsky, S.J.(1977a), on the scope of optimal and discretionary policies in the stabilization of stochastic linear systems, in J.D. Pitchford and S.J. Turnovsky (eds), Applications of Control Theory to Economic Analysis, Amsterdam: North-Holland Publishing Company.

Turnovsky, S.J. (1977b), Optimal control of linear systems with stochastic coefficients and additive disturbances, in J.D. Pitchford and S.J. Turnovsky (eds), Applications of Control Theory to Economic Analysis, Amsterdam: North-Holland Publishing Company.

Turnovsky, S.J., (1977c), Optimal monetary and fiscal policies in an open dynamic economy, Working Paper, Australian National University.

Turnovsky, S.J., Stabilization policies and the choice of monetary instrument in a small open economy, in A.R. Bergstrom, A.J.L. Catt, M. Peston, B. Silverstone (eds), Essays in Honour of Professor A.W. Phillips, London: Wiley, forthcoming.

Turnovsky, S.J. and J.D. Pitchford, Expectations and income claims in the generation of inflation, in A.R. Bergstrom, A.J.L. Catt, M. Peston, B. Silverstone (eds), Essays in Honour of Professor A.W. Phillips, London: Wiley, forthcoming.

Tustin, A. (1952), An engineers view of the problem of economic stability and economic regulation, Review of Economic Studies, vol. 19, 85-89. 
Tustin, A. (1953), The Mechanism of Economic Systems, London: William Heinemann Ltd.

Walsh, P. and J.B. Cruz (1976), Neighboring stochastic control of an econometric model, Annals of Economic and Social Measurement, vol. 5, Spring, 211-221.

Watkins, B.O. (1969), Introduction to Control Systems, N.Y.: Macmillan.

Waud, R.N. (1973), Proximate targets and monetary policy, Economic Journat, vol. 83, March, 1-20.

Waud, R.N. (1975), Information lags and the interest rate as a proximate monetary policy variable, Oxford Bulletin of Economics and Statistics, vol. 37, May, 103-113.

Wells, G. (1977), Approximately optimal macroeconomic stabilization policy: An application, Paper presented at the Sixth Conference of Economists, Hobart.

Witsenhausen, H.S. (1974), On the uncertainty of future preferences, Annals of Economic and Social Measurement, vol. 3, January, 91-94.

Wood, J.H. (1967), A model of Federal Reserve behaviour, in G. Horwich (ed) Monetary Processes and Policy: A Symposium, Homewood, Illinois, Richard.D. Irwin.

Zerby, J.A. (1969), An econometric model of monetary interaction in Australia, Australian Economic Papers, vol. 8, December, 154-177. 